\title{
MENISCUS MOTION \\ AND DROP FORMATION IN INKJET PRINTING
}

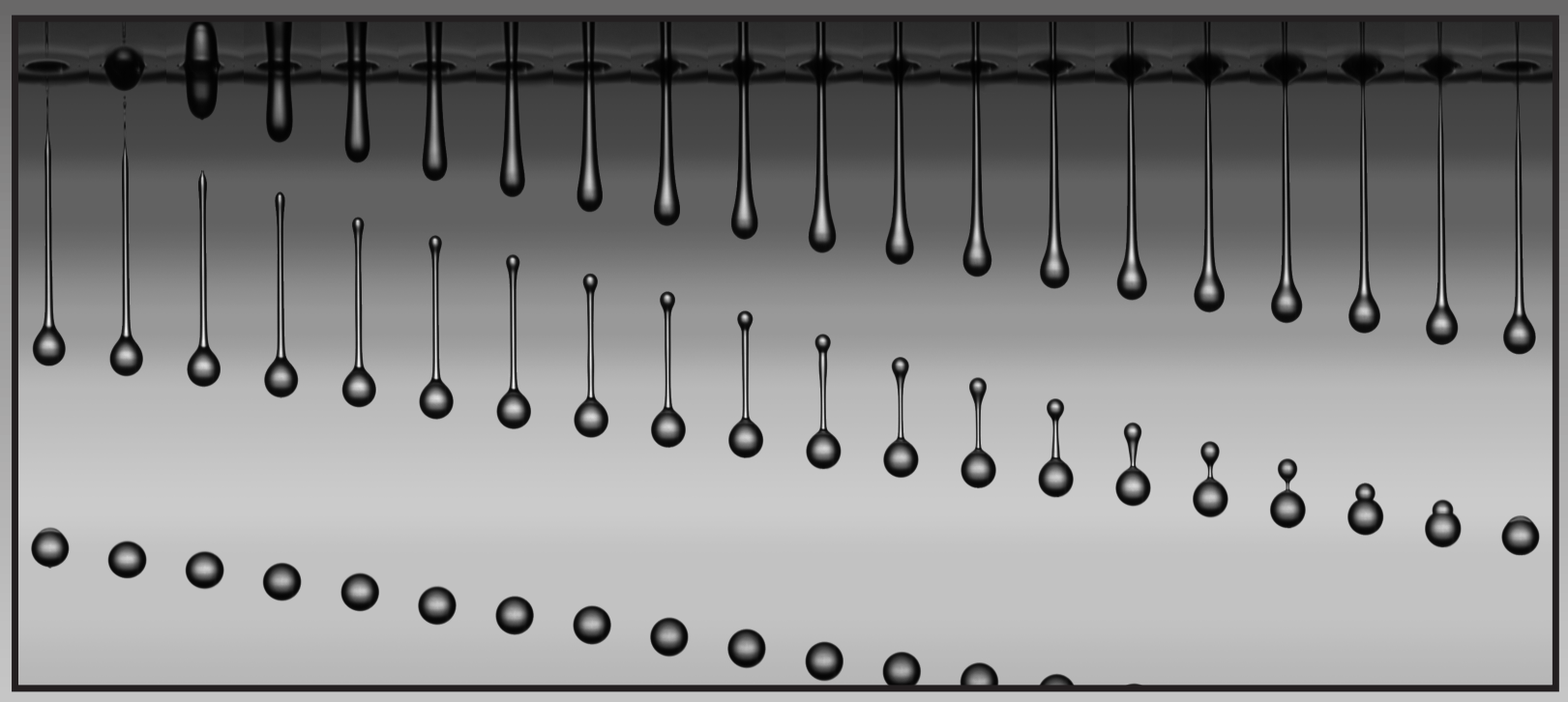

Mark-Jan van der Meulen 
Meniscus motion and drop formation in inkjet printing

Mark-Jan van der Meulen 


\section{Samenstelling promotiecommissie:}

Prof. dr. Hans Hilgenkamp (voorzitter) Prof. dr. Detlef Lohse (promotor)

Prof. dr. Michel Versluis (promotor)

Prof. dr. Jan Eijkel

Prof. dr. André de Boer

Prof. dr. Anton Darhuber

Prof. dr. Harald van Brummelen

Dr. Herman Wijshoff

Ir. Hans Reinten
Universiteit Twente, TNW

Universiteit Twente, TNW

Universiteit Twente, TNW

Universiteit Twente, EWI

Universiteit Twente, CTW-TM

Eindhoven University of Technology

Eindhoven University of Technology

Océ Technologies BV.

Océ Technologies BV.

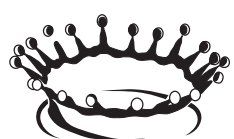

PHYSICS OF FLUIDS

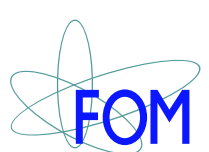

A CANON COMPANY

The work in this thesis was carried out at the Physics of Fluids group of the Faculty of Science and Technology of the University of Twente. It is part of the research programme Contact Line Control during Wetting and Dewetting (CLC) of the Foundation for Fundamental Research on Matter (FOM), which is financially supported by the Netherlands Organisation for Scientific Research (NWO). The CLC programme is co-financed by Océ Technologies BV., Venlo and ASML Netherlands BV, Veldhoven.

Nederlandse titel:

Inkjet printen: Meniscusbeweging en druppelvorming

Cover image: selected as Nature Magazine Image of the Year 2014.

Publisher:

Mark-Jan van der Meulen, Physics of Fluids, University of Twente,

P.O. Box 217, 7500 AE Enschede, The Netherlands

pof.tnw.utwente.nl

m.p.vandermeulen@alumnus.utwente.nl

(C) Mark-Jan van der Meulen, Enschede, The Netherlands 2014

No part of this work may be reproduced by print photocopy or any other means without the permission in writing from the publisher

ISBN: 978-90-365-3828-2ＤOI: dx.doi.org/10.3990/1.9789036538282 


\section{MENISCUS MOTION AND DROP FORMATION IN INKJET PRINTING}

\section{PROEFSCHRIFT}

ter verkrijging van

de graad van doctor aan de Universiteit Twente, op gezag van de rector magnificus,

Prof. dr. H. Brinksma, volgens besluit van het College voor Promoties in het openbaar te verdedigen

op donderdag 19 februari 2015 om 16.45 uur

door

Mark-Jan Pascal van der Meulen

geboren op 1 februari 1986

te Apeldoorn 
Dit proefschrift is goedgekeurd door de promotoren:

Prof. dr. rer. nat. Detlef Lohse

en

Prof. dr. Michel Versluis 


\section{Contents}

1 Introduction 1

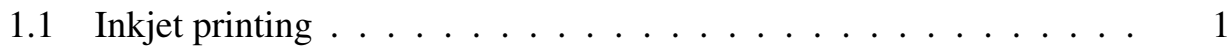

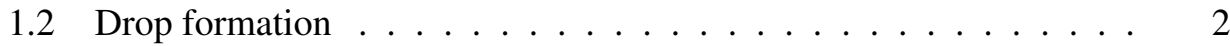

1.3 Experimental challenges in inkjet drop formation research . . . . 3

1.4 Guide through this thesis . . . . . . . . . . . . . 4

2 Experimental techniques for flow information in inkjet nozzles 5

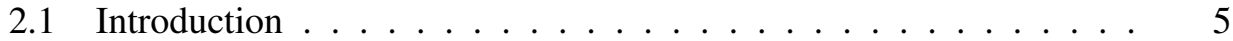

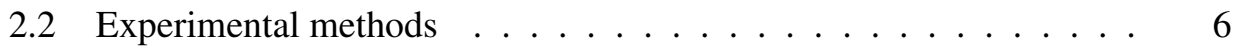

2.3 Discussion . . . . . . . . . . . . . . . . 25

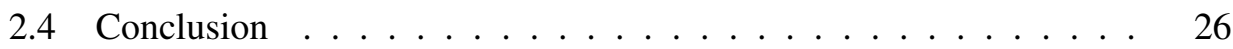

3 Non-axisymmetric effects in piezo-acoustic inkjet printing 29

3.1 Introduction . . . . . . . . . . . . . . . . . . . . . . 29

3.2 Theoretical approach to the meniscus instability . . . . . . . . 35

3.3 Experimental investigation . . . . . . . . . . . . . 41

3.4 Results . . . . . . . . . . . . . . . . . . . . 43

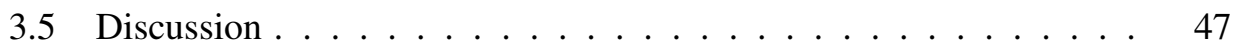

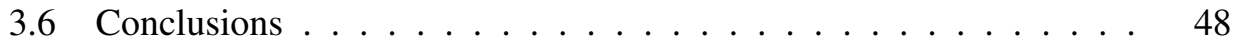

3.A High frequency print head $\ldots \ldots \ldots \ldots$

4 Characterization of a ballistic energy generator 53

4.1 Introduction . . . . . . . . . . . . . . . . 54

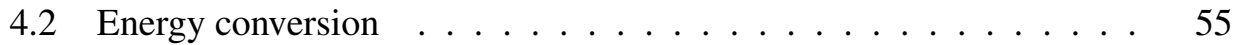

4.3 Electrical characterization $\ldots \ldots \ldots \ldots \ldots$

4.4 Energy conversion losses . . . . . . . . . . . . . . . . 62

4.5 Discussion . . . . . . . . . . . . . . . . . . . . 74

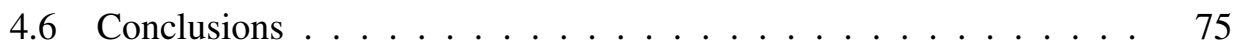

5 Velocity profile inside piezo-acoustic inkjet droplets 77

5.1 Introduction . . . . . . . . . . . . . . . . . . 77

5.2 Experimental setup . . . . . . . . . . . . . . 78 
5.3 Experimental methods $\ldots \ldots \ldots \ldots \ldots$

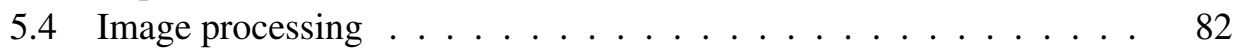

5.5 Droplet volume . . . . . . . . . . . . . . . . . . . 83

5.6 Droplet velocity . . . . . . . . . . . . . . . . . . 87

5.7 Validation of the experimental methods . . . . . . . . . . . . . 89

5.8 Conclusion \& Outlook . . . . . . . . . . . . . . . . . . . . 93

6 Drop formation CFD Benchmark 95

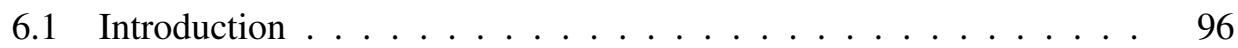

6.2 Benchmarks cases . . . . . . . . . . . . . . . . . . 98

6.3 Numerical cases . . . . . . . . . . . . . . . . . . . . . . . 107

6.4 Comparison . . . . . . . . . . . . . . . . . . . 108

6.5 Conclusions \& Outlook . . . . . . . . . . . . . . . . . . 122

6.A Edge detection of the microdroplets . . . . . . . . . . 123

6.B Drag reduction in drop trains . . . . . . . . . . . . . 126

7 Conclusions and Outlook 131

7.1 Conclusions . . . . . . . . . . . . . . . . . 131

7.2 Outlook . . . . . . . . . . . . . . . . . . . . 134

$\begin{array}{ll}\text { Bibliography } & 135\end{array}$

$\begin{array}{ll}\text { Summary } & 147\end{array}$

$\begin{array}{ll}\text { Samenvatting } & 149\end{array}$

$\begin{array}{ll}\text { Acknowledgements } & 153\end{array}$

$\begin{array}{ll}\text { About the author } & 157\end{array}$ 


\section{1 \\ Introduction}

\subsection{Inkjet printing}

Inkjet printing is a material depositing process in which small quantities of a liquid are expelled though a small nozzle, to form droplets that land on the substrate. The method excels in the fact that there is no contact required with the substrate and that the digital nature allows for a personalized production. The technique is well-known for the application of document printing. However the potential reaches further as becomes clear in the increasing popularity of the 3D-printing $[1,2]$ and many other digital fabrication industries [3].

Many reviews have been written on inkjet printing [4-6]. The technique is divided in two main branches: continuous inkjet printing (CIJ), were a continuous jet breaks up in a stream of drops [7] and where the required drops are subsequently selected, and Drop on Demand (DOD) inkjet printing were an actuation of the print head leads to the formation of a single drop. The most common DOD actuation methods are: thermal inkjet through the creation of a vapor bubble with a resistive heater, which sets the fluid into motion [8] and piezo electric inkjet using a piezo electric material which vibrates upon actuation, which sets the ink into motion [9].

The demand for increased feed-trough and smaller drop sizes continues to drive this field of research and seeks the potential challenges and limitations of inkjet technology. The work in this thesis aims at improving, and creating methods to improve and to understand small scale droplet formation. 


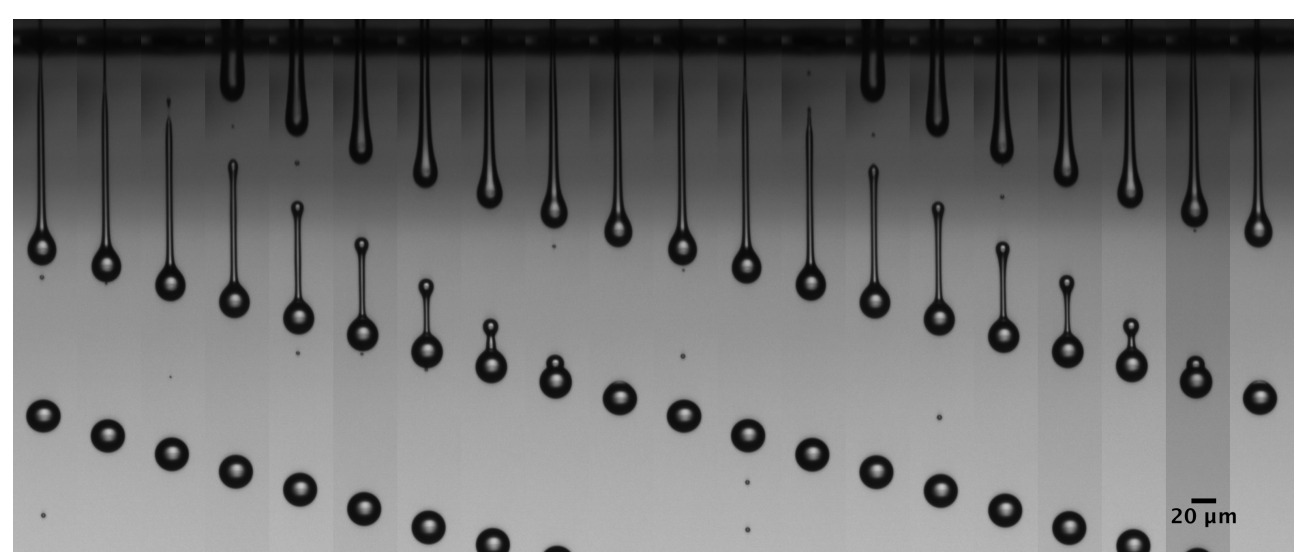

Figure 1.1: Stroboscopic droplet sequence with $5 \mu$ s time steps. A typical inkjet drop formation of $10 \mathrm{pL}$ droplets with a speed of $3 \mathrm{~m} / \mathrm{s}$, jetted at a DOD frequency of $20 \mathrm{kHz}$ with an Océ prototype print head.

\subsection{Drop formation}

Figure 1.1 shows a typical inkjet drop formation with $10 \mathrm{pL}$ droplets, jetted with a piezo electric prototype print head of the company Océ Technologies BV. The figure is composed of distinct droplets recorded at different times within the droplet formation cycle, with $5 \mu \mathrm{s}$ time delay steps. After ejection, the droplets have a spherical head drop connected to a very thin filament. The spherical head drop has a typical radius of $10 \mu \mathrm{m}$ while the cylindrical tail has a radius of 2-3 $\mu \mathrm{m}$. For ideal jetting conditions, the droplet contracts into a spherical drop. The very small drops in between the main drops are called satellite drops and have a typical size near $1 \mu \mathrm{m}$. They arise from Rayleigh breakup of the thin liquid thread that forms when the main drop pinches off from the fluid meniscus within the nozzle.

The units $[\mu \mathrm{m}]$ for the radius and $[\mathrm{pL}]$ for the drop volume are typically used in drop formation studies, and their geometrical relation, for spherical droplets, is plotted in Fig. 1.2. As a reference also the typical volumes for two important types of piezo electric printheads used in the present thesis are indicated, an Océ prototype print head and the AD-K Microdrop pipette. The droplet size found for DOD inkjet printing is typically the same size as the nozzle radius. 


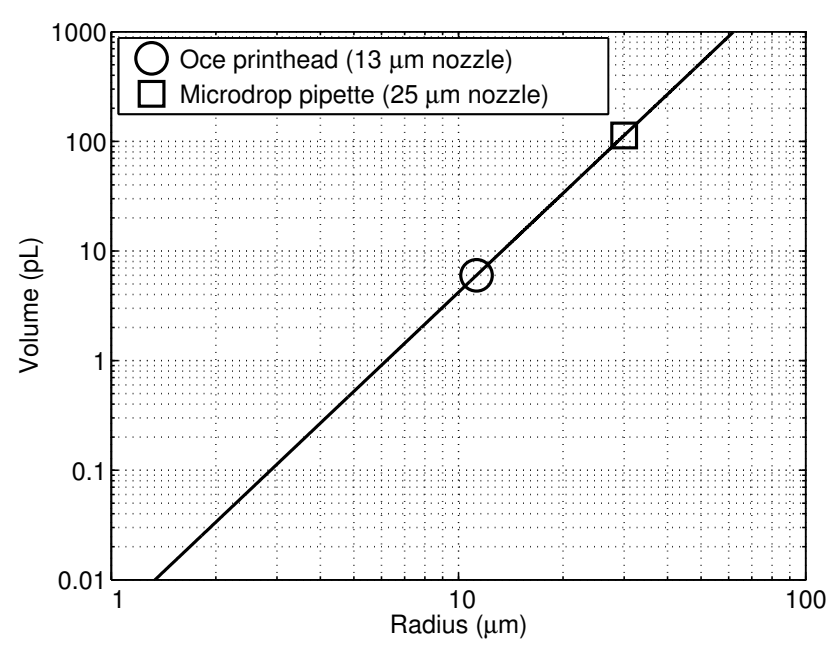

Figure 1.2: Volume dependence on the radius for spherical drops.

\subsection{Experimental challenges in inkjet drop formation re- search}

Experimental investigation of inkjet microdroplets is challenging, not only because of the tiny drop sizes, but also because the droplets move at high speeds of up to $20 \mathrm{~m} / \mathrm{s}$. To obtain combined high spatial resolution and drop recordings without motion blur, recordings at nanoseconds time scale is required. For example resolving the $2 \mu \mathrm{m}$ tail with a resolution of 10 pixels at a velocity of $10 \mathrm{~m} / \mathrm{s}$ demands a maximal illumination time of $20 \mathrm{~ns}$. This would require ultra high-speed imaging of 50 million frames per second [10]. State-of-the-art high-speed cameras are capable of decreasing the illumination time to the order of $100 \mathrm{~ns}$, but combined with the high magnifications involved and the amount of light required, this easily results in a substantial decrease of the resolution. To match these high optical resolution demands we make use of illumination by light induced fluorescence (iLIF) [10]; a laser light pulse of $7 \mathrm{~ns}$ is coupled into a fluorescent diffuser to illuminate a highly sensitive megapixel CCD or CMOS camera. The excellent reproducibility of the drop formation in inkjet printing allows for the construction of a time sequence from multiple different drops that are jetted in succession and recorded with an increasing delay.

One of the very important questions is the determination of the contour of the microscopic drops; the finite wavelength of light, diffraction of the light through the drops, the optical transfer function of the objective and the precise optical pathway of the emitted source light results in a characteristic blurred edge for the recorded head droplets and tail alike. Most of this information is unknown or proprietary 
or cannot be quantified. We approach the problem from an experimental point of view and quantify the droplet shape and its contour from physical quantities such as conservation of volume and the weighing of a large collection of droplets.

Finally, it is of great scientific interest to quantify the physical properties inside of the micro-sized nozzles, to learn how the contact line moves, to quantify the dynamical shape of the meniscus during a jetting cycle and if and how bubbles are entrapped. The small size of the nozzle together with the curved fluid meniscus make this a highly challenging and especially tedious job for inkjet printing research.

\subsection{Guide through this thesis}

The thesis builds up from investigations of the inkjet nozzle towards that of the droplets in free flight. To obtain information about the motion of the ink, or the shape of the ink surface (meniscus) inside the inkjet nozzle, different experimental techniques have been proposed and applied in the past decades. There is, however, still much to gain in this field. Chapter 2 reviews the experimental work done on the investigation of the nozzle and summarizes results of newly explored techniques and some of their potentials. There are many steps in the droplet formation process, that upon failure lead to jetting defects. These defects range from misaligned drops, strong satellite drop formation to even complete nozzle failure without drop formation. For most cases these defects can be attributed to physical defects within the nozzle, such as bubbles, wetting defects or misalignment of print head parts. Chapter 3 focuses on the question whether or not asymmetric drops can be formed in nozzles without defects. When asymmetrical shape instabilities, or surface modes, grow on the fluid meniscus during activation of the print head, they can give rise to asymmetrical drop formation. Experimental investigation of the meniscus verifies that the jetting of droplets works in a regime were these instabilities can arise and have sufficient time to grow.

The process of microdroplet formation can also be used in systems which convert mechanical into electrical energy. In Chapter 4 , such a device is investigated and the volume en velocity of the spherical droplets expelled from the nozzle are recorded using a double illumination technique to obtain aerodynamic loss mechanisms of charged droplets in flight. In Chapter 5 the potential of the high quality iLIF images is applied to the analysis of the volume distribution within droplets in free flight. Using a double frame recording technique, the flow within the microdrops is extracted and verified with a numerical droplet formation model using the slender jet approximation [11, 12]. In Chapter 6 this method is refined and then applied to a comparison of a set of popular numerical models for the prediction of drop formation dynamics. This effort allows for a direct comparison of the numerical predictions to the experimentally measured results of the drop evolution in time. 


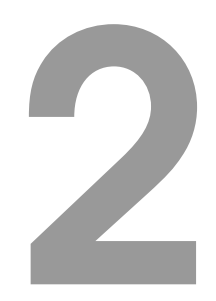

\section{Experimental techniques for retrieving flow information from within inkjet nozzles *}

In an inkjet print head the internal acoustics set in motion by a pressure pulse either caused by an exploding bubble or a piezo, and the droplet formation meet in the nozzle. The acoustics in terms of response in the frequency and time domain and the resulting droplet formation processes are well modeled and characterized by various experimental techniques. The behavior of the fluid in the nozzle is a critical mediator between these regimes, poorly accessible by experimental techniques. The meniscus shape and motion differ between print head designs, electrical pulse shapes and wetting conditions. The last decades several approaches have been proposed and implemented to experimentally obtain information on the fluid motion within inkjet nozzles. Here experimental methods are reviewed and novel techniques are introduced. The methods are compared in terms of accuracy and applicability.

\subsection{Introduction}

A good understanding of the drop formation process is important for improving the print quality of inkjet systems, such as straightness, droplet placement accuracy, constant droplet volume and speed, no satellites (or well controlled satellites, that either coalesce with the main droplet or land in the puddle of the main droplet on the sub-

*To be submitted as: Mark-Jan van der Meulen, Hans Reinten, Frits Dijksman, Michel Versluis, Detlef Lohse, Experimental techniques for retrieving flow information from within inkjet nozzles. 
strate). In drop-on-demand (DOD) inkjet printing the print head is actuated by introducing a pressure wave inside the ink channel. The pressure wave is created by the rapid formation of a bubble in thermal inkjet printing $[8,13]$ and with a piezoelectric actuator in piezo acoustic printing [9]. The formation and translation of pressure waves traveling back and forth inside the print head channel are well understood and are described with non-linear acoustical models [14-17], and can alternatively be predicted with CFD codes [18].

After the pressure wave has reached the nozzle, the meniscus is extended enormously and a jet of ink leaves the nozzle, and at some moment in time pinches off from the fluid connected to the nozzle. The fluid jet develops under action of surface tension, viscosity and the drag of the surrounding air into a drop and sometimes into a train of droplets. The formation of the microdroplets has been described theoretically $[19,20]$ and different droplet formation models have been proposed over the recent years: [12,21-26]. These models have been verified with high speed imaging of the microdroplets in flight, i.e. when outside of the nozzle [27]. Other techniques used to record droplets outside of the print head are Laser Doppler Anometry (LDA) [28] and holographic imaging [29].

The region where the acoustic and free flight domains are connected is inside the print head nozzle. Here the pressure wave energy is converted to kinetic energy and surface of the droplet. Unlike in continuous inkjet printing (CIJ), in DOD inkjet printing the fluid meniscus retracts in and extrudes out of the nozzle during a droplet formation cycle. The manner in which this energy is transferred strongly depends on the temporal shape, position and velocity of the meniscus in the nozzle, as-well as the pinning behavior of the meniscus at the nozzle wall. It is very challenging to experimentally test the predictions of the models in this region, since inkjet typically uses a small nozzle radius of $10-30 \mu \mathrm{m}$, the meniscus moves with velocities up to $20 \mathrm{~m} / \mathrm{s}$ and is easily exposed to accelerations $>10^{5} \mathrm{~g}$. Another issue to consider is what happens with the meniscus and the fluid directly after the meniscus, during idling. During the periods the printer is switched off maybe volatile components of the ink evaporate changing the physical properties of the ink and the droplet formation characteristics. This chapter reviews a number of published experimental techniques that have been applied to obtain information from within the inkjet nozzles, as well as a number of novel techniques. The methods are grouped in the directly measured physical quantities or the coupled relevant quantities of the fluid in the nozzle, i.e. the meniscus position and the velocity, pressure and volume in the nozzle.

\subsection{Experimental methods}

Figure 2.1 summarizes the quantities and the relevant methods to obtain them. In each of the cartoons the nozzle walls and fluid meniscus are depicted, together with 
the measured quantities in blue and the used medium or tools in red. To determine the velocity of the fluid in the nozzle Particle Image Velocimetry (PIV) can be used (Fig. 2.1(a)), tracer particles are dispersed in the ink which follow the flow. From the movement of these particles the velocity of the flow in the nozzle can be determined. The velocity of the fluid meniscus can be found using Laser Doppler Velocimetry (LDV) (Fig. 2.1(b)), the reflection of a laser pulse at the meniscus is interfered with a reference beam to find the Doppler shift caused by the movement of the meniscus. The volume in the nozzle can be determined using capacitive sensing with electrodes at the nozzle walls, (Fig. 2.1(c)). The varying ink volume in the nozzle changes the capacitance between the electrodes, which can be measured using the electrodes. The pressure of the fluid in the nozzle can be determined using piezo electric sensing (Fig. 2.1(d)). The compression and expansion of the piezo electric material is directly converted into an electrical signal. Finally to measure the position and shape of the meniscus there are three methods, reflectional imaging, imaging through a transparent nozzle and 3D confocal microscopy using tracer particles (Fig. 2.1(e-g)). With the reflectional imaging technique, the reflection of light at the meniscus is interpreted. The reflections give rise to a specific intensity patterns, from which the shape of the meniscus can be determined. When the nozzle is optically accessible, the position and shape of the meniscus can be recorded using shadow photography. With the last method the shape of the of the meniscus can be determined using tracer particles in the ink. By locating the positions of the particles the fluid surface can be outlined. Scanning through the depth of the nozzle reveals the 3D shape of the meniscus.

At the end of this chapter these techniques are discussed in terms of information that is extracted, its accuracy, advantages and disadvantages. 


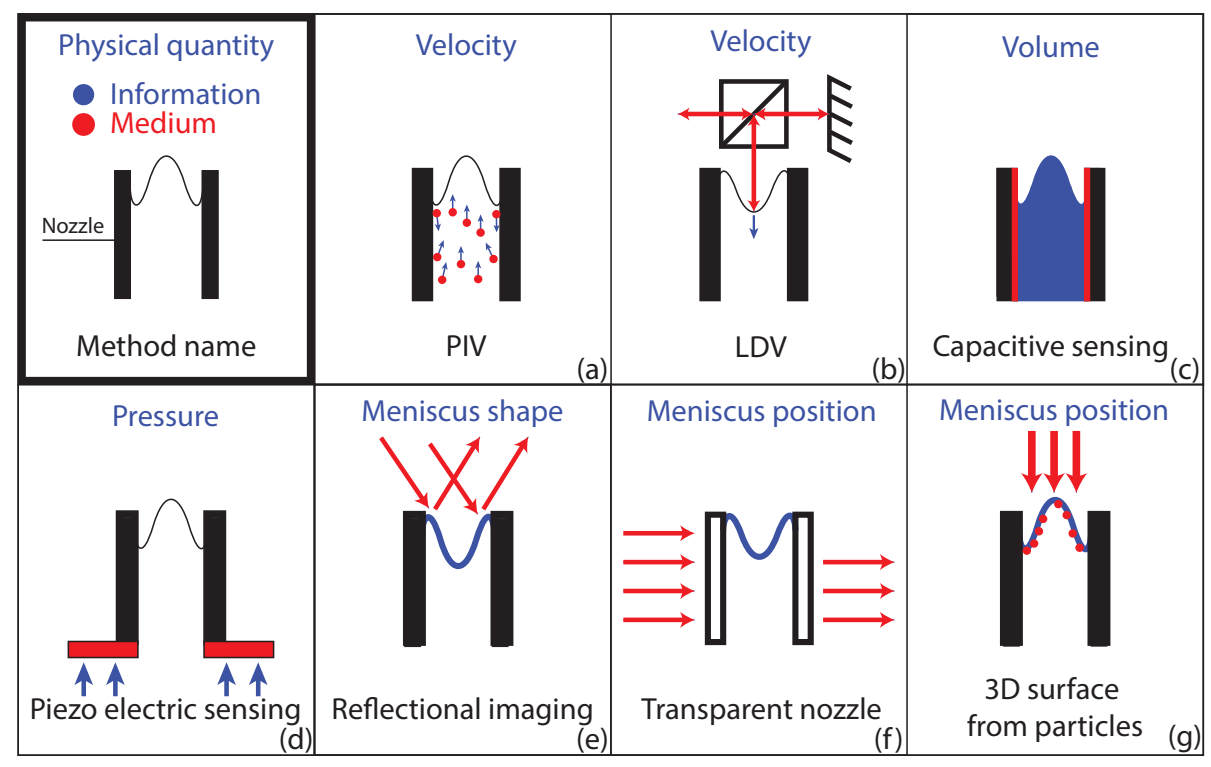

Figure 2.1: Overview of experimental techniques for obtaining information from within inkjet nozzles. The sketch summarized which physical quantity can be obtained from within the inkjet nozzle, which is indicated by the black bars. The blue in the figure indicates the quantity which is determined, and in red the medium or tool to obtain the quantity is indicated.

\subsubsection{Velocity}

The velocity of the ink in the nozzle is a consequence of the actuation pressure, the print head geometry, and the fluid properties of the ink such as viscosity, surface tension and wetting. The initial shape and velocity of the meniscus will have a strong influence on the final drop velocity. To be able to predict how a specific actuation of the print head results in the velocity of the meniscus, reliable measurements are necessarily.

\section{PIV in nozzle}

Particle image velocimetry (PIV) [30] is a technique to estimate the velocity field in a fluid from the displacement of tracer particles between subsequent recorded frames. The flow domain is divided in interrogation windows, which are convoluted over the neighbouring frames to find the most likely displacement of the fluid in the time between the frames. The fluid domain which is analyzed needs to be optically accessible for the visualization of the particles. Velocities obtained from particles near a wall, 
i.e. within approximately $2-5$ times the particle diameter may be inaccurate due to the particle/wall interaction [31]. The spatial resolution of the method depends on the size of the interrogation windows, which is chosen in such a way that each window contains at least four tracer particles. However the size of the interrogation windows must be chosen small enough such that the flow field within the window is uniform.

Meinhart et al. [31] were the first to report PIV experiments in an inkjet print head nozzle and chamber. They used $700 \mathrm{~nm}$ fluorescent particles in a SEAJet print head with a $30 \mu \mathrm{m}$ radius nozzle. The plexiglass sidewalls allowed for fluorescence imaging inside of the print head. From the particle positions they also extracted the instantaneous meniscus position, see Fig. 2.2(a). Making use of the reproducibility of the inkjet process to phase-average over multiple drop formation cycles they obtained a resolution of $4 \times 11 \mu \mathrm{m}$ (using $50 \%$ interrogation window overlap) with a depth of $8-10 \mu \mathrm{m}$. They reached an accuracy of $2 \%$ of the full scale velocity. They also measured a flow focussing in the nozzle, with a maximum velocity of $8 \mathrm{~m} / \mathrm{s}$ in the centre of the nozzle.

Castrejón-Pita et al. [32] applied high-speed PIV to a MicroFab print head with a transparent tapered glass nozzle and nozzle exit diameter of $80 \mu \mathrm{m}$. They recorded $2 \mu \mathrm{m} \mathrm{TiO}_{2}$ particles at a frame rate of $5 \times 10^{5}$ frames/s using a high speed camera, see Fig. 2.3. They did not make use of a laser sheet to illuminate the particles, but recorded the particles with back-illumination, thereby capturing all the particles in the $200 \mu \mathrm{m}$ depth of field of the microscope. This combination resulted in a radiallyaveraged velocity from the PIV algorithm. They were able to resolve $6.5 \mu \mathrm{m}$ (using $50 \%$ window overlap) interrogation windows with a depth of $200 \mu \mathrm{m}$. Because of the high speed imaging, they were able to resolve a full actuation cycle in realtime. The tapered shape of their print head induces an optical distortion due to the refractive index differences of glass and air, and it was found that the radial direction in the print head is overestimated by $50 \%$. On the other hand, they found that the correction on the axial flow direction is negligible on the micrometer scale, since the refractive indices of the glass and the liquid almost match. The velocity profile during a complete actuation cycle is as plotted in Fig. 2.3(b). A typical velocity of $2 \mathrm{~m} / \mathrm{s}$ on the centre axis in the nozzle was observed. Castrejón-Pita et al. hinted that non-transparent nozzle can be accessed using ultrasonic or X-ray particle velocimetry.

\section{Laser doppler vibrometer}

In Laser Doppler Vibrometry (LDV) the velocity of a moving object can be determined by interference of a reflected laser beam with a reference beam. This technique allows for a very high velocity resolution. The movement of the object causes a Doppler frequency shift in the reflected beam, which can be determined from the interference pattern of the two beams. The velocity is calculated from the observed frequency-shift with $v=\frac{\lambda \Delta f}{2}$. By aiming the laser beam perpendicular to the nozzle 


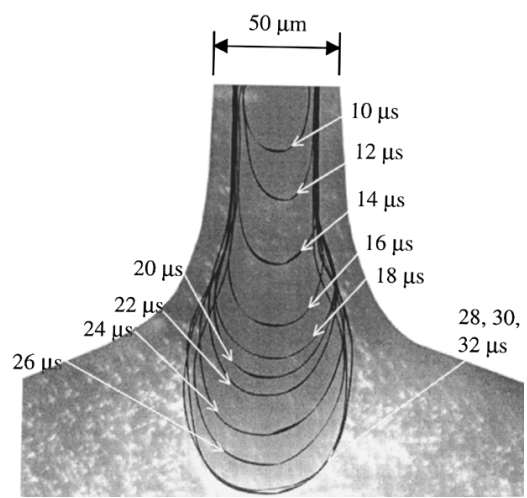

(a)
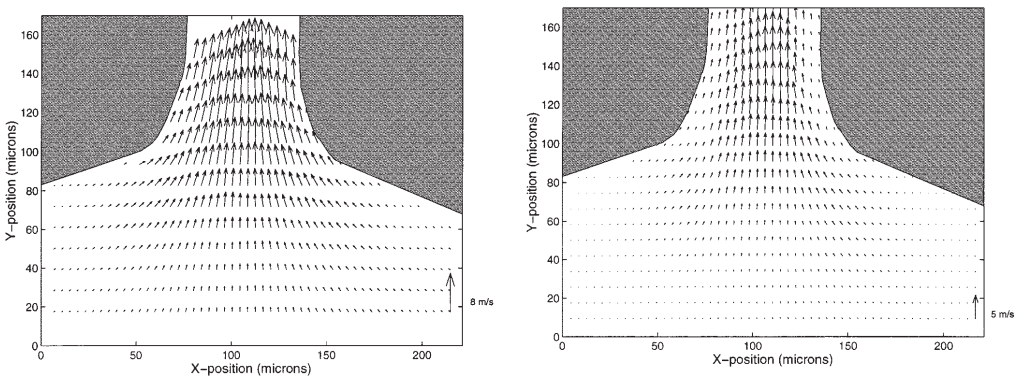

(b)
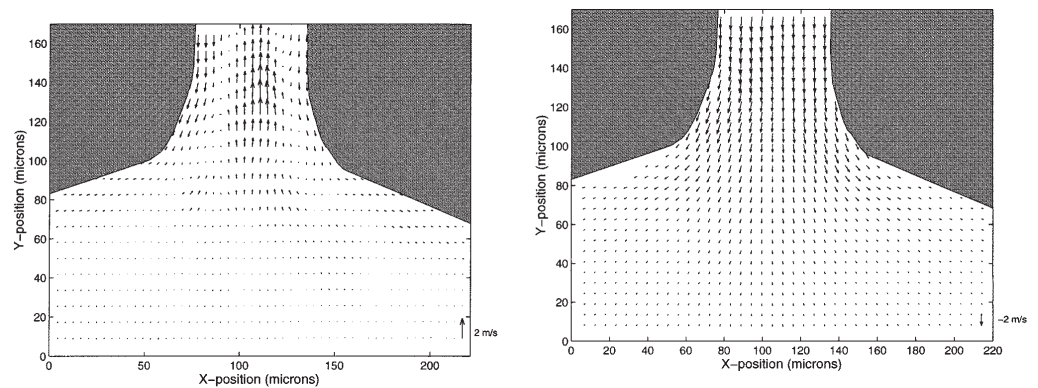

(c)

Figure 2.2: PIV recording of Meinhart et al. [31] with micro particles in a $30 \mu \mathrm{m}$ radius transparent nozzle (SEAJet). (a) The black lines represent the instantaneous position of the meniscus at the indicated time steps. (b) At $70 \mu$ s the flow is directed outwards of the meniscus, at $80 \mu$ s the flows rate is decreased. (c) At $85 \mu$ s flow reversal takes place in the nozzle. At $90 \mu$ s the flow is completely inwards of the nozzle. 

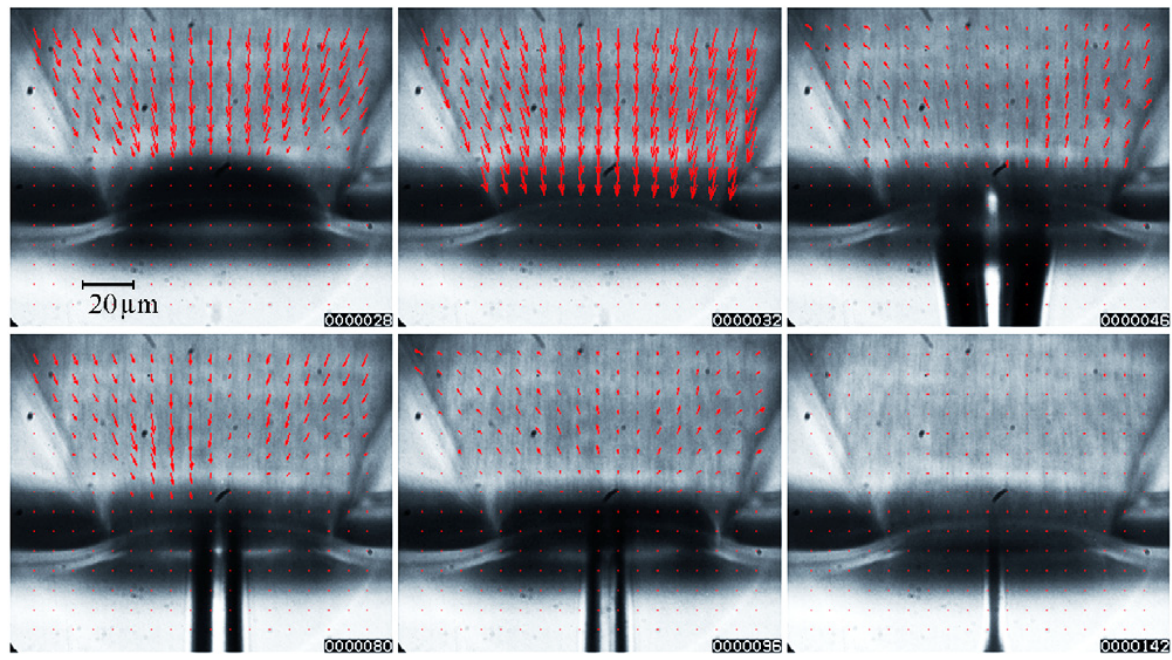

(a)

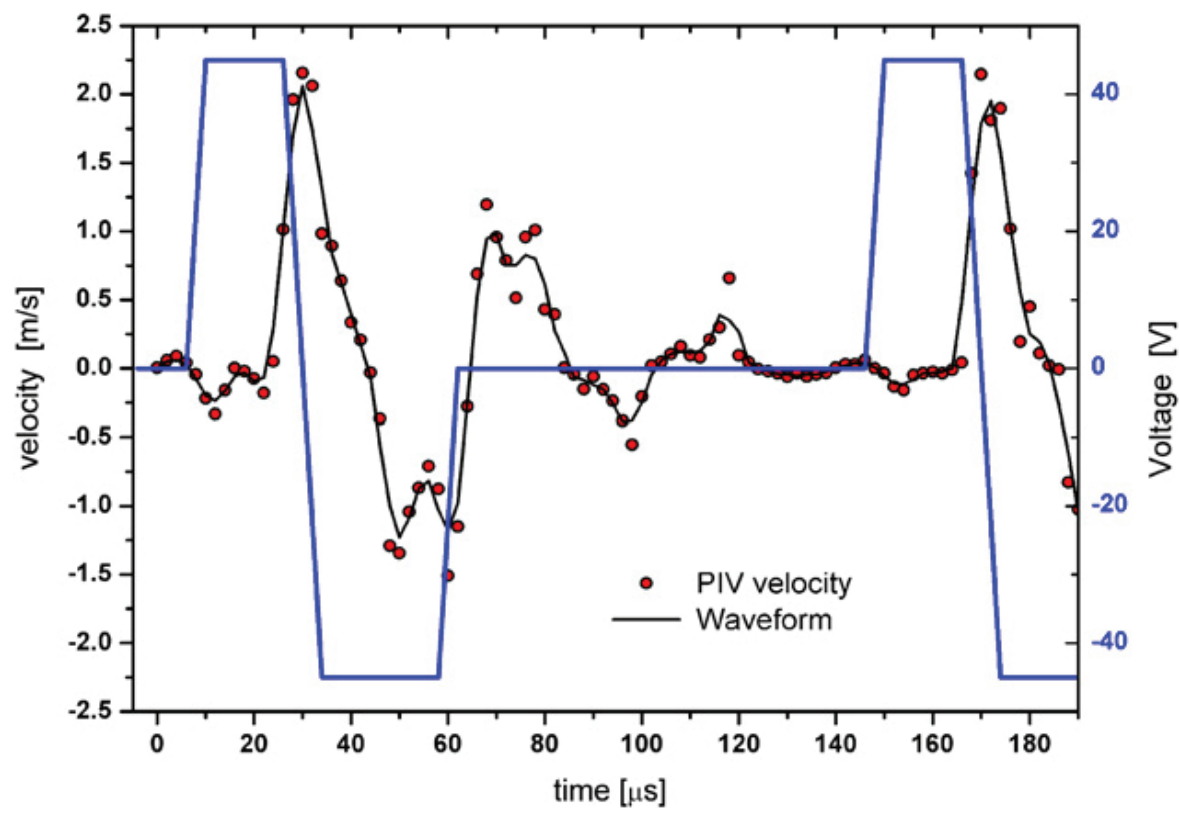

(b)

Figure 2.3: PIV experiments in glass tapered nozzle (MicroFab ( $25 \mu \mathrm{m}$ radius), performed by Castrejón-Pita et al. [32]. (a) The particles are recorded with back-light illumination. The image recording time shown in $\mu$ s at the bottom right corner of each frame is offset by $140 \mu \mathrm{s}$ from the triggering of the first drop. The scale bar indicates the observed magnified scale inside the nozzle which is $50 \%$ enlarged w.r.t. the scale outside the nozzle. (b) Time variation of the liquid velocity as measured by PIV within the nozzle on its axis. The velocity profile is fitted with a spline curve. 
plate, i.e. into the nozzle, the velocity of the meniscus can be found in a single point on the meniscus. The maximum velocity is bounded by the carrier frequency of the system, and ranges typically from $3-30 \mathrm{~m} / \mathrm{s}$ [33]. The spatial resolution is limited by the laser spot size, which can be reduced to a few micrometers using microscopy, and experimental resolutions down to $0.5 \mu \mathrm{m} / \mathrm{s}$ were achieved [34].

One of the limitations of this technique is the fact that the probing laser beam has to be reflected back to the objective, and since the meniscus is curved, the beam is easily scattered to different directions. This technique is therefore easiest applied in the centre of the meniscus, which under normal operating conditions is approximately flat. For optimal perpendicular viewing the laser beam is positioned in the drop path, the drop then has to be deflected after creation, which causes a time window in which recordings are not possible. Viewing the nozzle under an angle can overcome this problem, however the changing shape of the meniscus will introduce an additional error in the measurement. Also for a large numerical aperture (N.A.) of the objective, the incoming angle of the focussed light cone is large. This incoming laser light beam will then be blocked by the nozzle walls when focussing at a meniscus which is retracted further than approximately one nozzle diameter. This results in further loss of light, and as well as a decrease of the effective N.A.

C.C. Poon et al. [35, 36] used LDV to study the refill time of the nozzle and crosstalk effects on the velocity of actuated nozzles with a $25 \mu \mathrm{m}$ radius. They used a TSI Model 9236 optical vibrometer in combination with a $15 \mathrm{~mW}$ HeNe laser. The laser beam waist was focussed to a $14 \mu \mathrm{m}$ radius and was directed at the nozzle at an angle of $12.5^{\circ}$, see Fig. 2.4. The amount reflected of reflected light at the meniscus was only $2 \%$, but a stronger signal was obtained from reflections at the nozzle plate, which decreases the quality of the signal. Further depreciations of the signal were caused by inhomogeneities in the ink and the changing position due to the evolving shape of the meniscus. To increase the signal-to-noise ratio the velocity measurements were averaged over 256 actuations. In their work on the nozzle refill time, they measured a velocity over a complete jetting cycle and determine what was called, the reduced velocity. This is the many cycle averaged velocity, which is lower than the maximal velocity of the meniscus. Also the variation from actuation to actuation and the change in the meniscus shape can change the measured velocity. From the measured refill time they are able to optimize the jetting frequency of the print head. In their work on crosstalk the meniscus oscillations in a non-actuated nozzle caused by actuated nearby nozzles was measured and also the influence of the velocity of the meniscus due to the presence of neighboring actuated nozzles.

Seo et al. [37] used LDV in the centre of the meniscus of a piezoelectric print head. They investigated the drying of water-based black pigment inks in an inkjet head with stacked piezo elements, using an Onosokki: LV-1710 LDV. Small actuation probe pulses were applied to make the meniscus oscillate below $10 \mathrm{~m} / \mathrm{s}$, causing 


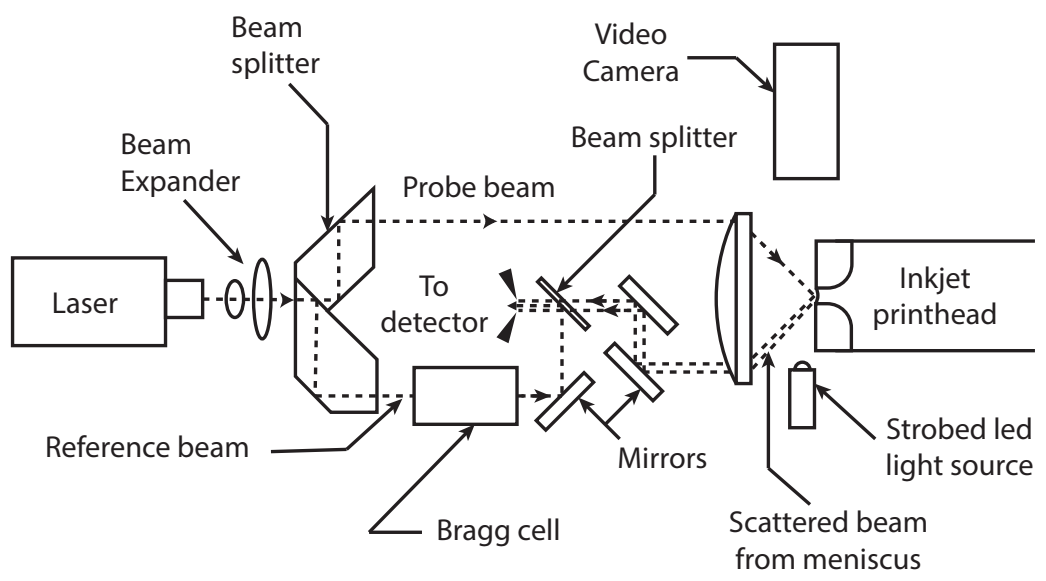

(a)
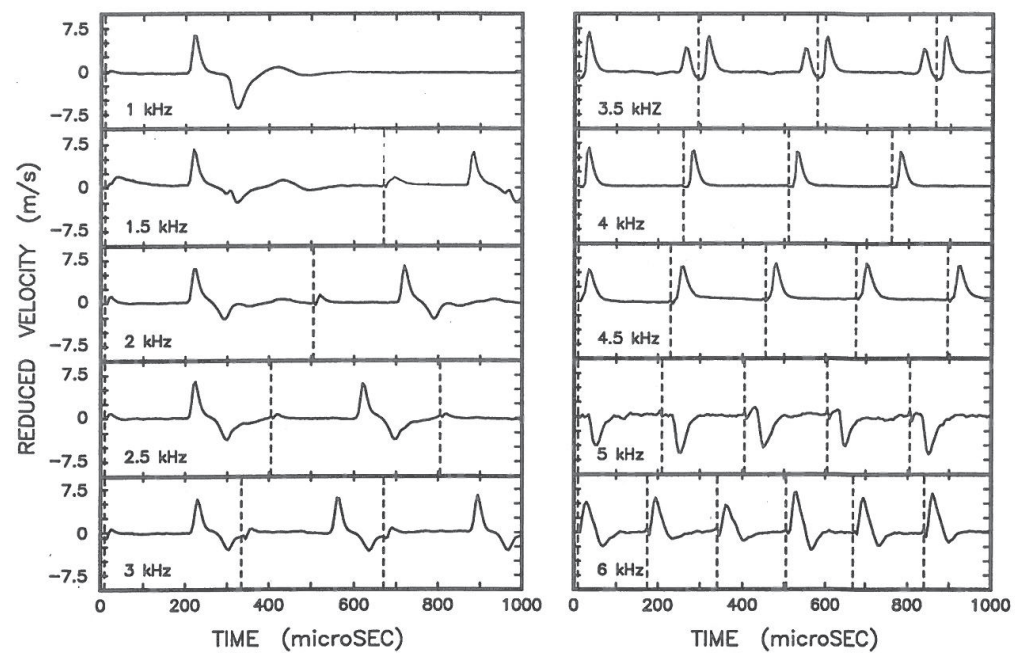

(b)

Figure 2.4: (a) LDV setup used by Poon et al. [35]. The incoming beam is directed at an angle $18^{\circ}$, the refracted bream is interfered with the reference beam. (b) Measure velocity signal [36]. The dotted lines indicate the excitation of the bubble jet. The first maximum in the signal indicates the emerging of the meniscus, which followed by retraction of the meniscus. When the DOD frequency is increased the next velocity maximum starts to interact with the moving meniscus. This causes increased or reduced drop volumes. 
movement of the meniscus of about $15 \mu \mathrm{m}$. From the LDV measurements the velocity of the meniscus during the damping oscillations was obtained. Coupling this to a lumped mass model, allowed to estimate the viscosity change of the ink due to evaporation.

In the present study scanning-LDV experiments have been performed. LDV signals are recorded at multiple places of the meniscus and the reproducibility of the meniscus movement allows for the reconstruction of the 3-dimensional meniscus movement. For this experiment a Polytech: MSA-400 LDV was used which has a spatial resolution of $1.2 \mu \mathrm{m}$. The used print head was a single nozzle drop dispenser (Microdrop), with a nozzle radius of $25 \mu \mathrm{m}$, jetting water micro droplets. The LDV setup measures velocities up to maximal $10 \mathrm{~m} / \mathrm{s}$, which is easily exceeded during normal jetting conditions. Therefore measurements of small actuations were performed, after which the meniscus starts to oscillate. The probing beam in the experiment was directed perpendicular to the nozzle plate at different positions in the nozzle and the reference beam was reflected at the nozzle plate to reduce distortion of small print head motion on the meniscus velocity signal. The typical noise in the velocity signal was only $5 \times 10^{-3} \mathrm{~m} / \mathrm{s}$. In Fig. 2.5 (a) a time series measured at the center of the nozzle is depicted. On top of the main meniscus movement a higher frequency velocity oscillations is visible. Similar movements have been observed for stronger actuations with optical side view recordings. Numerical integration of the velocity signal gives an estimation of the position of the meniscus. Figure. 2.5(b) indicates the reconstructed height profile. The scanning measurement area was larger than the nozzle area. The glass nozzle plate was wetted somewhat near the nozzle and a small wave was observed after actuation of the print head. For stronger actuations it was not possible to quantify meniscus motion. 


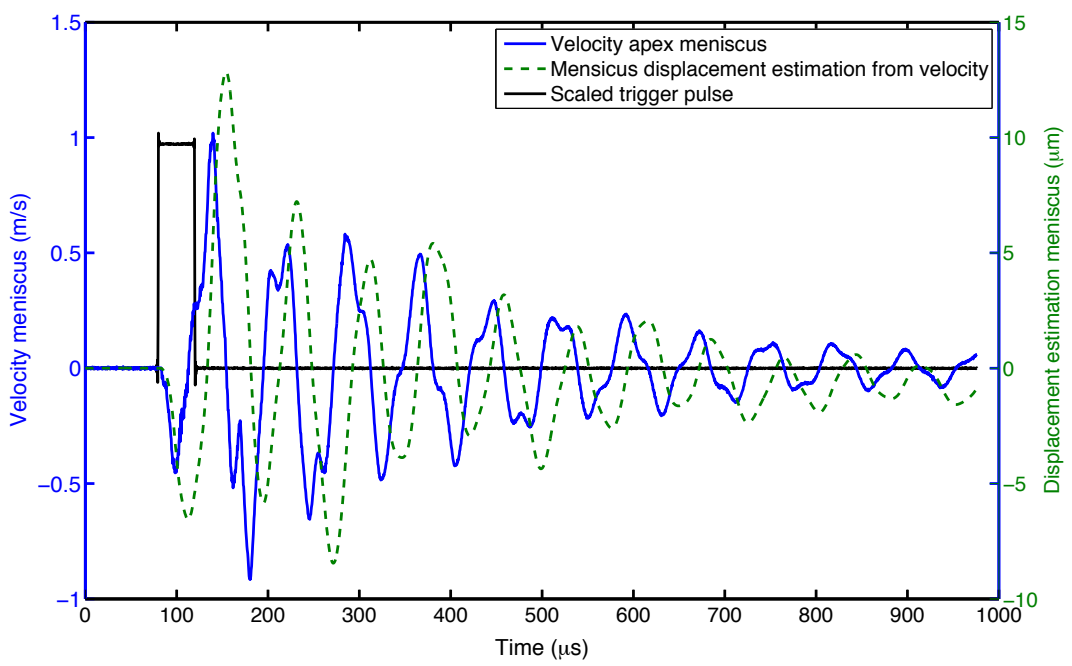

(a)

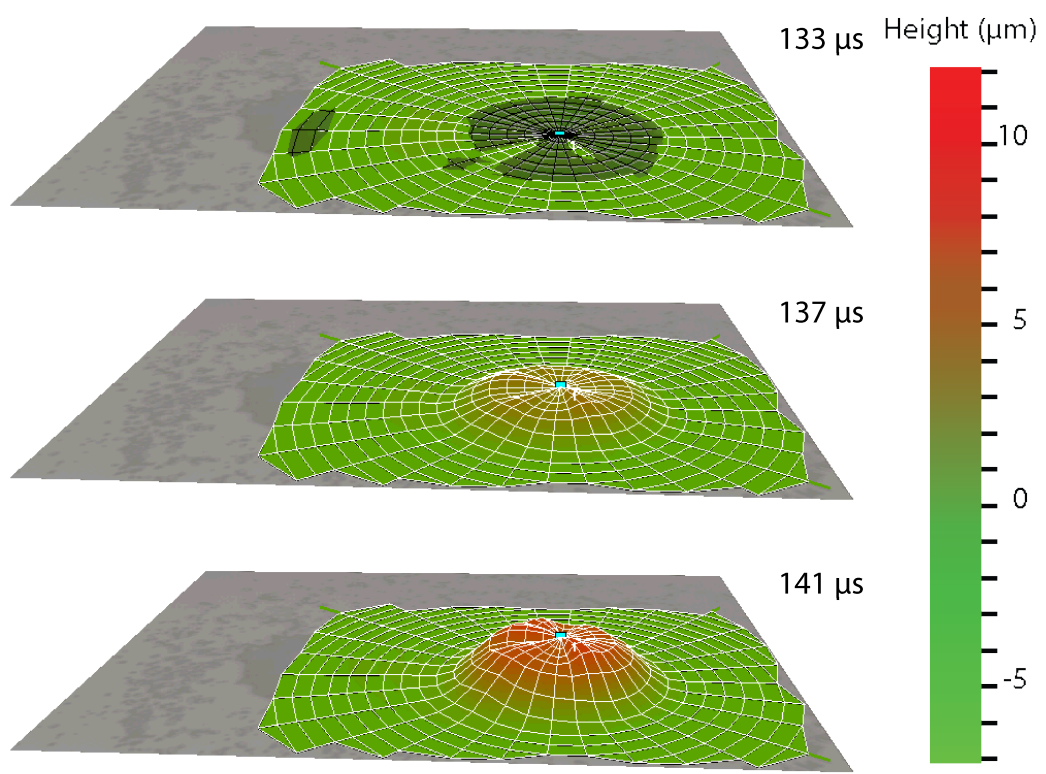

(b)

Figure 2.5: Scanning LDV measurements. (a) Velocity measurement and position calculation of apex of meniscus. The meniscus is oscillating after a small actuation pulse. (b) $3 \mathrm{D}$ height reconstruction from LDV scan of a $25 \mu \mathrm{m}$ nozzle. For all the points in the grid a time recording is made from which the respectively positional data is reconstructed. The scanned area is larger than the nozzle. 


\subsubsection{Volume}

The amount of ink volume in the nozzle at the moment that the actuation pressure wave arrives has, due to inertia, a strong influence on inkjet system, e.g. the ejected drop velocity and the temporal eigenfrequency of the print head. Experimentally obtaining the volume in the nozzle, during the actuation of a print head, therefore gives an improved insight in the manner inkjet drops are formed.

\section{Capacitive sensing}

When electrodes are placed alongside the inner nozzle wall, the volume change of ink inside the nozzle changes the capacitance between the electrodes. A volume change of $10-100 \mathrm{pL}$ results in a capacitive variation of a few femtoFarad. These small capacities can easily be distorted by electrical noise or parasitic capacitances present in the measurement system itself. Therefore this method requires a complex readout system to deal with the small signals received.

J. Wei et al. [38] designed and built a MEMS-produced cavity resembling an inkjet nozzle with integrated electrodes. An dedicated read out system based on lock-in-amplification was implemented on-chip to take care of the small signals and to prevent parasitic capacities. The evaluated nozzle is a $50 \mu \mathrm{m}$ long cylinder with a $20 \mu \mathrm{m}$ radius, see Fig. 2.6. The total capacity change from filled to empty, in the $63 \mathrm{pL}$ nozzle was measured to be $12.6 \mathrm{fF}$. Assuming a flat meniscus the resolution of the meniscus position corresponded to $32 \mathrm{~nm}$. The scalar nature of capacitance only allows for an estimate of the filling degree of the nozzle, not of the meniscus shape. Since the menisci in inkjet nozzles are usually curved and sometimes ranges outside of the nozzle, the measured capacitance values are not uniquely defined for a certain filling degree. The sensors have to be embedded in the nozzle, during the fabrication. Capacitive sensing allows for direct measurements during printing, which can be used in a closed loop control system. A jetting experiment with the chip attached to a Microdrop pipette have been performed, see Fig. 2.6(c), indicating that the system could respond at the short timescales of the meniscus movement. An increase of the signal was found, even when the meniscus extruded out of the nozzle.

\subsubsection{Pressure}

The pressure wave in the print head is a consequence of the actuation of the print head and results in the movement of the fluid in the nozzle. To test the quality of theoretical predictions on this pressure wave, experimental results are required. 


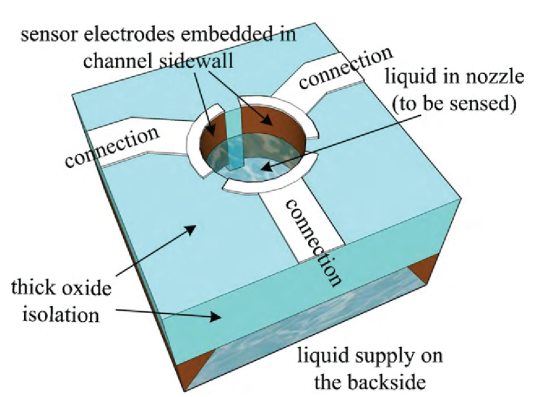

(a)

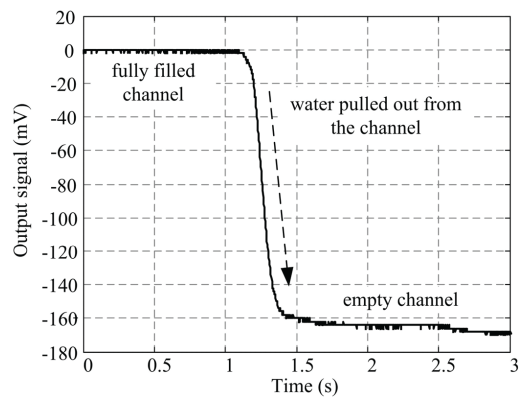

(b)

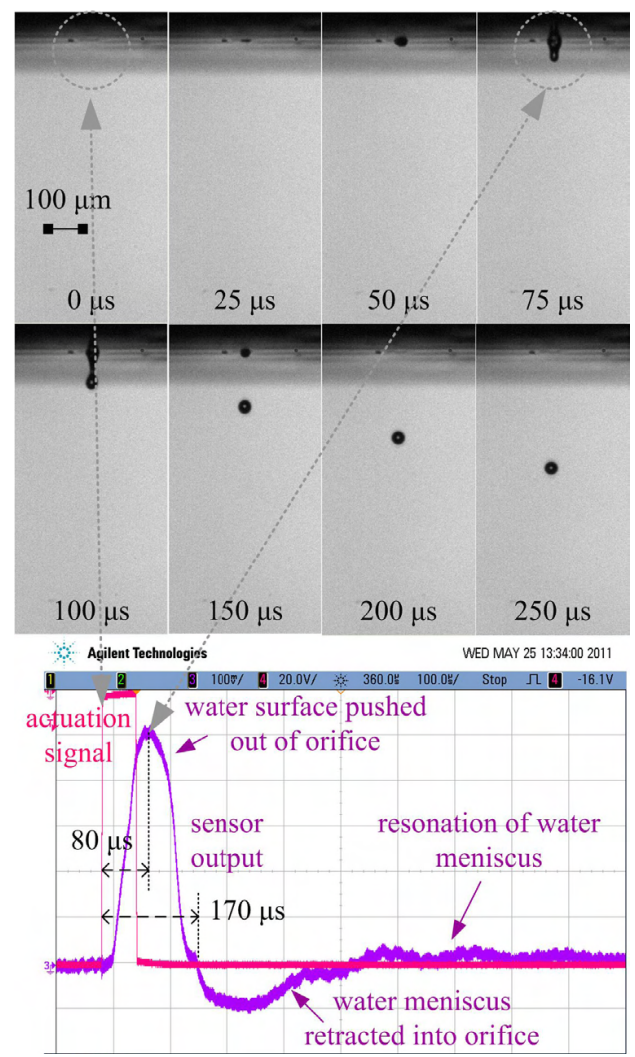

(c)

Figure 2.6: (a) Schematic view of the capacitive sensor in an inkjet nozzle. (b) Measured signal when the water is pulled out from the channel [38] , an output signal variation of $160 \mathrm{mV}$ is detected between the full en empty states. (c) Output of the sensor with the recorded camera images during the generation of a droplet [39]. The extrusion and retraction of the meniscus are detected well. The measured capacitance is found to slightly increase even when the meniscus is already protruding out of the nozzle. 


\section{Piezoelectric sensing}

The fluid in DOD inkjet print heads is actuated by the creation of a pressure wave, which travels to the nozzle to produce droplets. Piezoelectric sensors can be used to measure these pressure waves. The pressure changes during its travel to the nozzle due to compliance and the geometry of the print head channel. When the pressure near the nozzle is known, droplet formation models can be used to calculate the motion of the meniscus. A great advantage of this method is that online readout can be performed during the inkjet printing process, thus giving a direct feedback during the printing process. The method requires the integration of the sensor during the production of the print head. Although it is possible estimate the response of the meniscus on the pressure wave, the exact shape of the meniscus predicted by the models depends on assumptions such as wetting conditions and pinning behavior of the meniscus in the nozzle.

J. Wei et al. [40] designed a geometry where the piezoelectric sensors are located around the nozzle. The nozzle plate is made from a $1 \mu \mathrm{m}$ thin membrane, made from a SOI wafer, which deforms during interaction with the pressure wave. In the design piezoelectric sensors were placed around a $10 \mu \mathrm{m}$ radius nozzle in the presence of tensile stresses due to the out of plane motion of the membrane. The compressional stresses near the edges of the membrane, can also be measured, however, this stress is much more sensitive to alignment errors of the nozzle and the ink chamber. For an applied pressure of $1 \times 10^{5} \mathrm{~Pa}$ a $8.7 \%$ variation of the $9.97 \Omega$ nominal resistance was measured. For the used configuration a sensitivity of $3.9 \times 10^{-7} \mathrm{~V} / \mathrm{Pa}$ was found.

For piezo-actuated print heads, the actuator itself can be used as a sensor in between the actuation cycles [41, 42]. Using the actuator as a sensor gives a pressure signal inside of the print head channel, which is different from the one directly at the nozzle. The pressure at the nozzle can be found by using a theoretical model [14]. Thus this method requires no adaptation of the standard piezo acoustic print heads, but involves more modeling to obtain information on the motion in the nozzle.

\subsubsection{Meniscus shape}

The meniscus is the interface which separates the fluid domain in the print head from the outside world. The specific shape of the meniscus in the nozzle before or during actuation, can result in variations of the droplet formation process. To better understand the drop to drop variations, more insight can be obtained from the actual meniscus during the actuation cycles.

\section{Reflectional imaging}

Light that reflects at a curved meniscus towards a camera, gives rise to a specific pattern of brighter and darker rings. Bright rings appear where light from the light 

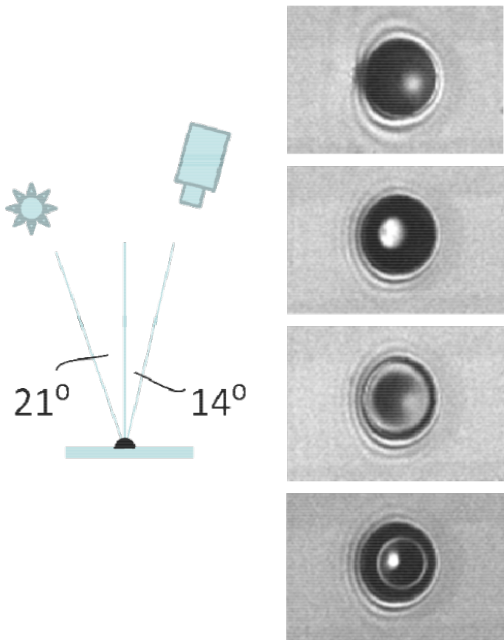
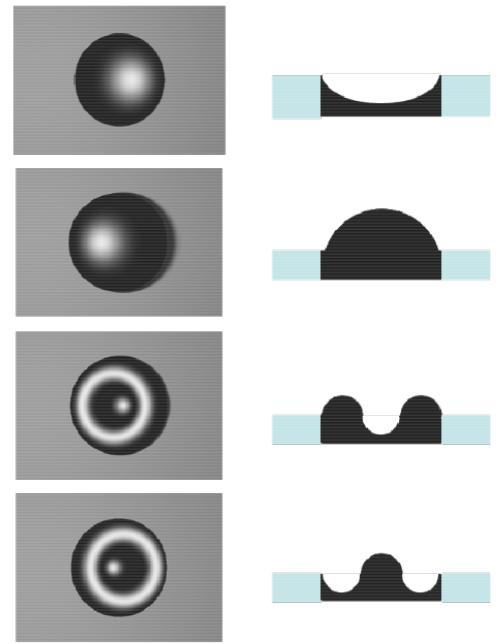
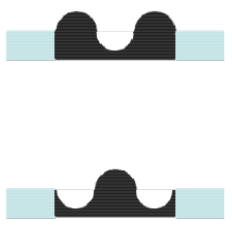

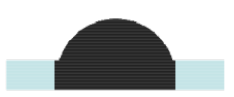

Figure 2.7: Reflectional imaging, from Hsiao et al. [43]. Left: Recording of the reflections from different meniscus shapes. Right: The rendered images from static shaped with ray tracing indicate the shape of the meniscus at the different meniscus shapes.

source is directly reflected onto the microscope camera. Evaluation of these light and dark rings give information on the temporal shape of the meniscus; the location of the bright areas indicate the normal of the surface and the width indicates the normal at which reflected light still falls within the aperture of the objective. Point sources and collimated light sources give better resolution than diffusive light sources.

Hsiao et al. [43] used a setup where the light source was directed under a $14^{\circ}$ angle and the camera under a $21^{\circ}$ angle with respect to the main axis of the nozzle plate of a Xaar print head. They found this configuration maximizing the illumination within their space constraints. Therefore the observed reflection rings are non-concentric for axi-symmetric meniscus shapes. By applying raytracing to a solid meniscus shape they where able to estimate the temporal shapes of the meniscus. In Fig. 2.7 several meniscus shapes have been shown together with the rendered images from ray tracing. Using a high-speed camera they were able to capture a complete actuation cycle of the meniscus. It was observed that the meniscus starts oscillating before the meniscus retracts into the nozzle and different meniscus shapes during the actuation cycle were observed. If the meniscus is retracted further into the nozzle, the nozzle edges blocked the light path of the incoming and reflected light rays, resulting in a black spot. With this technique they were able to study cross talk between nozzles. Since the camera is aimed at an angle at the nozzle plate, this system can also be used during normal printing conditions, but no useful information will be obtained for menisci deeply retracted into the nozzle. 
To obtain more quantitative information from this method, the illumination has to be controlled more precisely. Also the method could be expanded by combining light sources under different angles, for example with different color light sources and a high-speed color camera. Combining steeper and shallower illumination angles allows for simultaneously obtaining global meniscus shape information, as well as information on a deeper retracted meniscus.

\subsubsection{Meniscus position}

The meniscus position directly after an actuating cycle, reveals the refilling of the nozzle with ink. This is important for the print head actuation. For incomplete refilling of the nozzle with ink before a new actuation cycle is started, the resulting reduced volume in the nozzle will have a strong influence on the ejected droplet volume and velocity. Knowing the position of the meniscus during the actuation of the print head, results in a better insight of the refill times and the retraction of the meniscus.

\section{Transparent nozzle}

Although some of the previous techniques require a transparent nozzle, having a transparent nozzle allows for recording the meniscus in the nozzle directly with backlight imaging. The global shape of the meniscus can be extracted from these recordings over a full jetting cycle with either a high-speed camera or by high-speed time-resolved imaging. To have minimal distortion from refraction at the print head surface, a flat transparent side wall [31], is optimal. The typical cylindrical or conical shape nozzle of print heads, result in a distorted view inside the nozzle, which can be corrected for [44].

Experiments have been performed in a single nozzle print head of Microdrop with a tapered glass nozzle, see Fig. 2.8. The obtained image resolution outside of the nozzle is is $320 \mathrm{~nm} /$ pix. The image inside of the nozzle is stretched up to 1.5 times in the radial direction. The edge of the meniscus inside of the nozzle becomes visible in the recording, since the backlight is refracted by the water-air interface. In these recordings the air appears as a dark region, instead of the fluid. Focussing the image at the axis of the nozzle results in sharp edges near the axial meniscus boundaries of about $1.6 \mu \mathrm{m}$, but due to refraction the edge at the apex is blurred over substantial distance, see Fig. 2.8(a). The evaluated edge profile is changing with meniscus shape, and extracting the exact edge position at high precision is difficult and would require a more thorough analysis of the optical features of the nozzle and imaging system.

Moving the focus plane of the imaging system reveals a plane where the apex of the meniscus is sharper, and the meniscus apex boundary reduces to $8 \mu \mathrm{m}$. In this plane also more details about the meniscus movement are revealed, such as the inward curvature at the final time step of Fig. 2.8(b), and entrapped bubbles (data 
not shown). It is expected that the tapered cylinder of the nozzle acts as a lens by itself, which changes the focus within the nozzle. Since the meniscus boundary does not appear to be in focus for a single focus plane, the complete meniscus shape can not be determined from these single recordings. However it is possible to trace the position of the meniscus apex, see Fig. 2.8(c). The meniscus edge is estimated from the inflection of the intensity profile over the boundary, the accuracy of the meniscus position has been estimated to be $2 \mu \mathrm{m}$.

For a deeply retracted meniscus, i.e. more than one nozzle diameter, it is difficult to see whether the meniscus is still pinned at the nozzle plate, or that the pinning line is receding into the nozzle. This imaging concept can also be applied with infrared imaging, to study the inside of silicon print heads, which have a transmittance of $50 \%$ at in the near-infrared (NIR) band wave, but not transparent for visible light, as shown in [45]. This method has been applied to visualize bubbles inside an inkjet print head chamber [46], but could also be used to study the meniscus inside of this type of print head. The longer wavelength results in a typical reduction by a factor 2 in resolution, for the same N.A. 


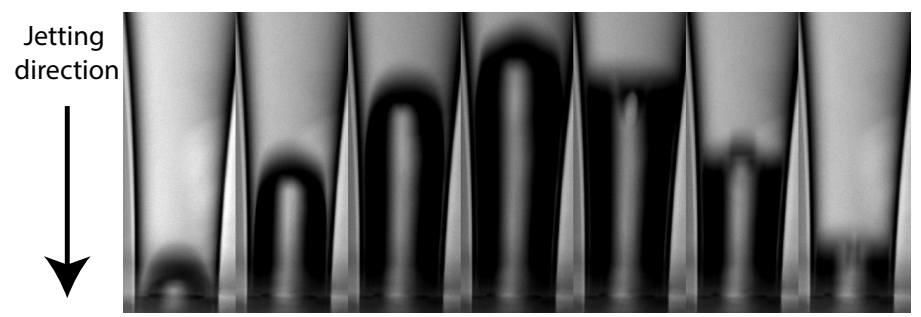

(a)

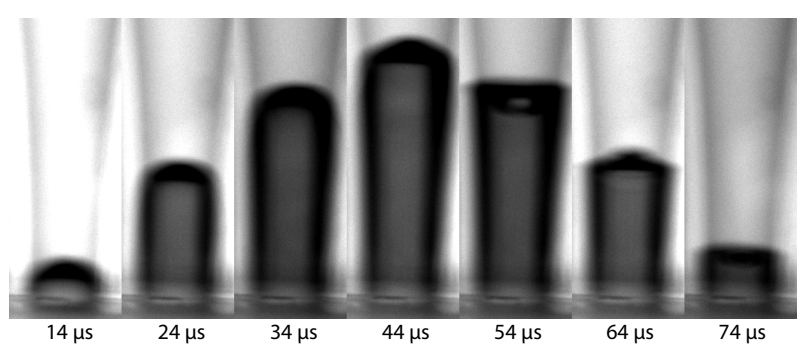

(b)

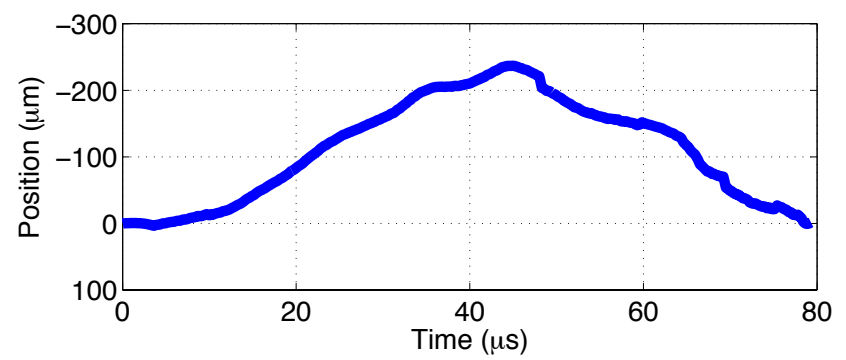

(c)

Figure 2.8: Recording meniscus trough transparent tapered nozzle with $25 \mu \mathrm{m}$ inner exit radius. (a) Focussed at central axis of the nozzle; the droplets outside of the nozzle and the nozzle walls are in focus. (b) Focussed at meniscus boundary; the nozzle wall is out of focus, but the tip of the meniscus inside the nozzle is imaged much sharper. (c) Trace of meniscus apex motion. 


\section{D confocal microscopy with tracer particles in ink}

Direct reflectional imaging reveals only the positions of the meniscus where the light is reflected from the meniscus back into the camera objective. When light is radiated from the fluid surface, this limitation is overcome and light from every non-obstructed part of the meniscus can be observed, in- and outside the nozzle.

Tracer particles which are dispersed in the ink can act as (fluorescent) light sources at the meniscus interface. By using an objective with a small focal depth, only a thin plane across the fluid meniscus lies in focus. Thus tracer particles that are in focus in this image plane are in the fluid. From these particle positions, the position of the fluid in this plane can be outlined, as indicated in Fig. 2.9(a). By exploiting the repeatability over multiple actuation cycles, the focus plane can be scanned from inside to outside of the nozzle revealing the position of the meniscus at all heights. Applying monochromatic illumination and fluorescent particles allows for the separation of the direct reflective light from the light of the tracer particles.

Experiments have been performed with $1 \mu \mathrm{m}$ fluorescent particles in the single nozzle print head of Microdrop which has a nozzle radius of $25 \mu \mathrm{m}$. An inverted fluorescence microscope equipped with an Olympus SLMPLN 50× objective, was used to image the nozzle area. The depth of field of the setup was $2.8 \mu \mathrm{m}$. The obtained meniscus shapes were compared at multiple time steps to a side view recording through the transparent nozzle. Only particles near the surface give reliable information on the position of the fluid interface, since the observed particle positions deeper in the fluid are shifted due to refraction. Once the shape of the meniscus is known however, the aberration can be corrected for. The meniscus could be visualized up to $70 \mu \mathrm{m}$ inside of the nozzle. Particles behind the steep angled surface of more elongated menisci are not observed since either the refracted light from the particles does not reach the objective or the laser light does not reach the particles, leaving only the flattened parts of the meniscus to be visualized. A reconstruction of the fluid volumes, obtained at different times after actuation are shown in Fig. 2.9(b). A lateral resolution of $7.8 \mu \mathrm{m}$ was obtained using the particle positions of 9 frames at the same time step. The resolution can improved by using phase averaging over more images, and/or using a larger particle density. A $1 \mu \mathrm{m}$ resolution is expected to be feasible. The amount of particles, however, must remain small since a high concentration of particles will cause clogging of the nozzle. A variation to this approach is using a fluorescent ink or dye in the ink. Using confocal microscopy the meniscus segments in and out of focus are separated. For this method the resolution per frame is not limited by the size of the particles, but by the microscope imaging resolution, eliminating the need for using more frames per time step. 


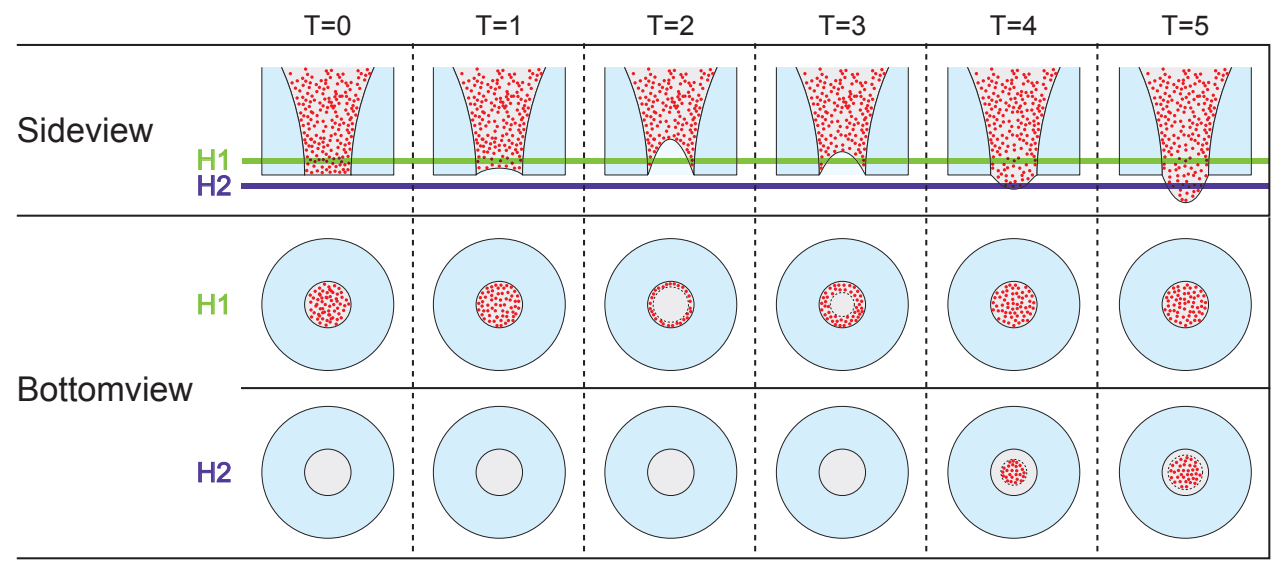

(a)

$0 \mu \mathrm{s}$

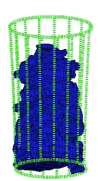

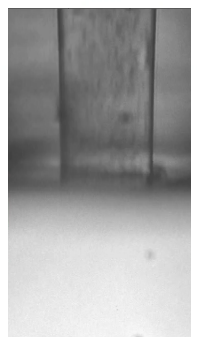

$10 \mu \mathrm{s}$
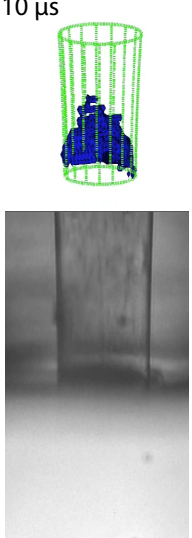

$20 \mu \mathrm{s}$
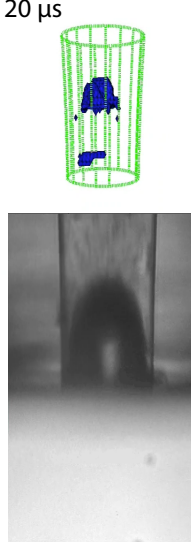

$30 \mu \mathrm{s}$
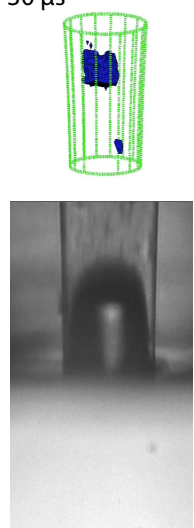

$40 \mu \mathrm{s}$
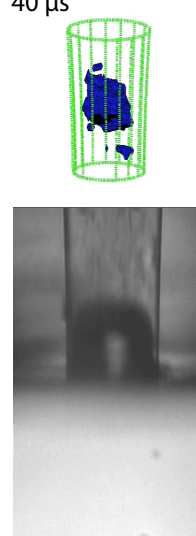

$50 \mu \mathrm{s}$
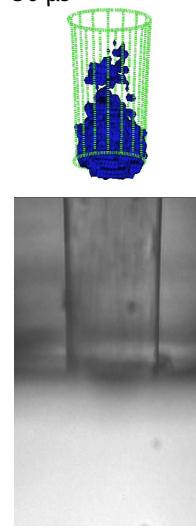

$-20 \mu \mathrm{m}$

(b)

Figure 2.9: Observed particle distributions from bottom view for different shapes of the meniscus. H1 is focussed inside and H2 is focussed outside of the nozzle. Stacking the information of different heights allows for a $3 \mathrm{D}$ reconstruction of the meniscus. (b) Comparison of reconstructed fluid volume of particles compared with side view recordings at the same moment in time after the actuation of the print head. The reconstructed resolution is limited in the current experiments, resulting in rough estimations of the fluid volume. 


\subsection{Discussion}

The collection of experimental techniques measure different physical parameters, such as: the velocity of the fluid inside the nozzle or the meniscus surface velocity, the filling degree in the nozzle or the shape of the meniscus. Not all methods can be used during actuation, where drops are formed. Sometimes they can, however, be applied outside the normal operating range with small meniscus oscillations. Other methods require additional adjustments of the print head itself. In Table 2.1 the comparison of the different techniques is summarized in terms of measured parameter, accuracy and applicability.

The first six listed techniques can be applied during normal inkjet printing conditions and as such in commercial machines, the use tracer particles might be unacceptable and the use of the microscope under an angle reduces the accuracy of some the measurements. Some techniques are only applicable as a research and development tool. For PIV measurements tracer particles are required and the ink must be transparent; when the ink is a suspension, these particles can be used as tracers, otherwise the particles have to be dispersed in the fluid which is often undesired. To use real time analysis of the nozzle during printing, the electrical methods, i.e. capacitive sensing and piezoelectric sensing, are the best choice, since the electrical signals can be analyzed directly without the need for image post-processing. The disadvantage of these techniques is that they do not measure the motion of the meniscus directly. The motion of the meniscus has to be determined through modeling efforts, which can be notorious inaccurate since they rely on assumptions such on the pinning and wetting behavior inside and at the rim of the nozzle. To obtain the meniscus shape in a nontransparent nozzle, LDV or shape determination from particles can be used. LDV has the highest resolution, down to $1 \mu \mathrm{m} / \mathrm{s}$, but can only be used for small actuations. This technique is therefore suitable to visualize crosstalk responses or the meniscus motion shortly before actuation. The use of transparent nozzles is a restriction on the choice of materials for the print head. The global shape of the meniscus can be extracted from these recordings, but it is difficult to determine or even estimate the exact pinning conditions of the meniscus at the nozzle wall. The shape determination from particles can be used in non-transparent nozzles and can give a good resolution down to $1 \mu \mathrm{m}$, but the technique requires a highly repeatable jetting process since the height scan requires multiple actuations per height step and multiple actuations are required to account for the low density of particles at the meniscus interface Also it is difficult to obtain an optical signal from stretched parts of the meniscus.

None of the experimental techniques discussed here are able to resolve the motion of the contact line of the meniscus, which is most interesting for the design of stable inkjet print heads. Since the fluid layer can become very thin during the retraction of the meniscus, the most likely way that this can be resolved is in terms of optical interferometry. 


\subsection{Conclusion}

Several experimental techniques have been discussed to obtain information on the fluid inside the micro-nozzle of inkjet print heads, including velocity, shape and position of the meniscus, the volume in the nozzle and the pressure near the nozzle. Previous works which have deployed these techniques have been reviewed and variations were proposed. Next to the works found in literature, results of three new techniques for investigating the inkjet nozzle are presented: scanning LDV of the fluid meniscus, meniscus shape determination using a transparent nozzle and using tracer particles.

With LDV the highest velocity resolution and spatial resolution (via numerical integration) is obtained. However it can only be applied in a small range, where the maximum velocity of the device is not surpassed and the meniscus is not retracted too far into the nozzle. Inside of the nozzle the most insights on bubbles and meniscus shapes are obtained from the use of transparent nozzles, but the tapered cylindrical nozzles make it challenging to determine the meniscus position with high precision. Capacitive and piezoelectric sensing detect a direct electrical signal, for which the sensors need to be integrated in the print head. But these signals can be processed and acted upon during the jetting process (closed loop control) and therefore these methods are very suitable for integration in commercial print heads. 


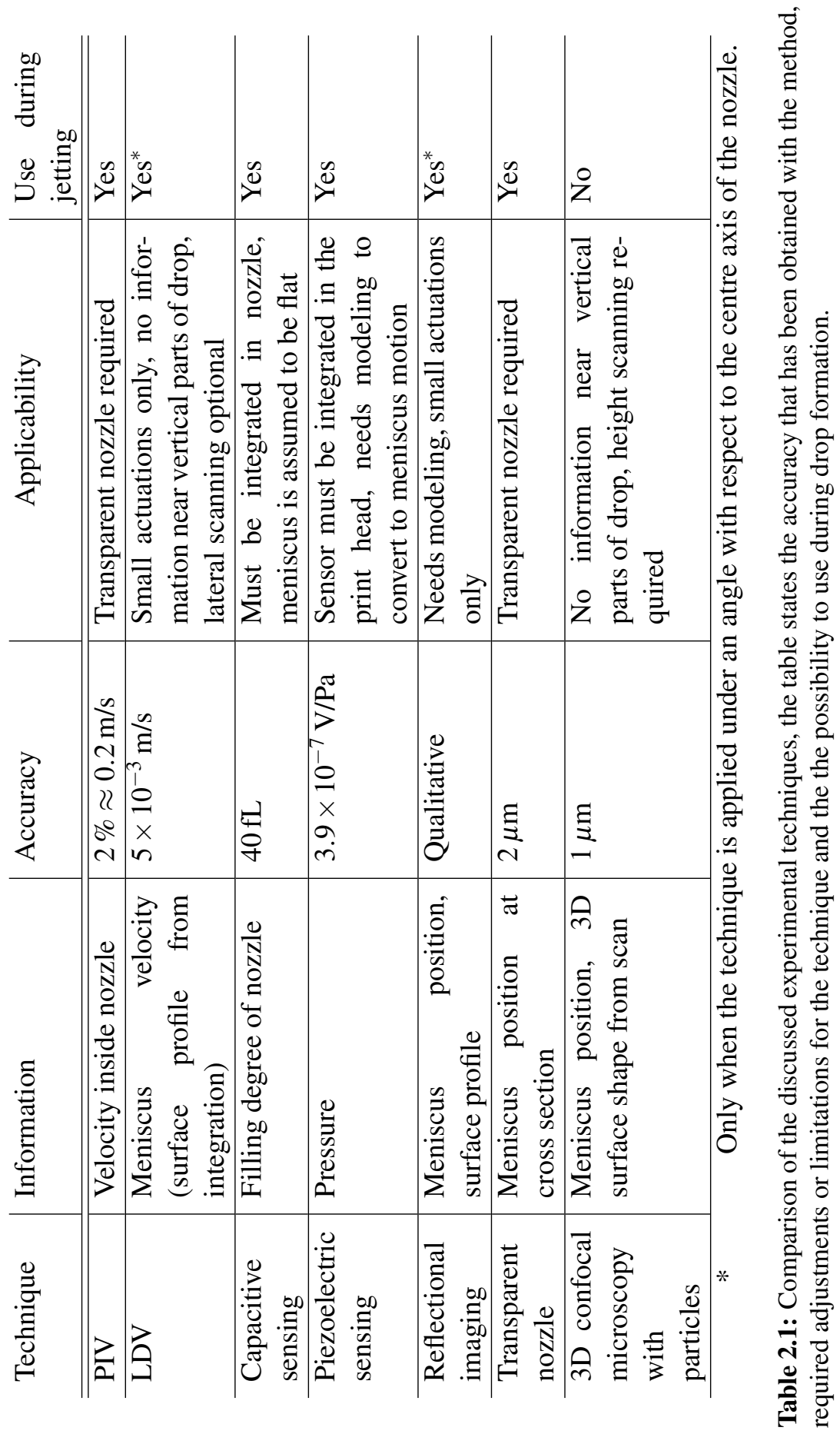




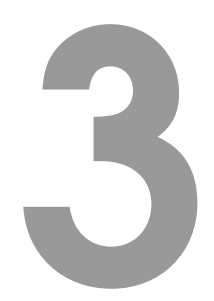

\section{Non-axisymmetric effects in drop-on-demand piezo-acoustic inkjet printing *}

Drop-on-demand (DoD) inkjet printing is well-characterized and a well-studied problem, but non-axisymmetric effects are typically ignored, while these effects can severely reduce the print head performance and its stability. In this chapter we first review non-axisymmetric droplet formation originating from geometrical effects. We then focus on the possibility that non-axisymmetric effects arise from surface instabilities of the meniscus; these include the Rayleigh-Taylor instability and a parametrically driven capillary instability. It is shown that the meniscus can become unstable beyond a critical acceleration threshold. A comparison with data extracted from high-speed recordings of the meniscus oscillations show that the acceleration threshold can indeed be exceeded, with higher order temporal frequencies exhibiting even larger accelerations. The experiments show that the duration the acceleration thresholds are exceeded, are of the same order as the typical growth times of the instabilities.

\subsection{Introduction}

Drop on Demand (DoD) inkjet printing is a well established non-contact material deposition method which is best known for usage in the graphical industry. The great advantage of DoD ink-jet printing is that material is only deposited where and when

*To be submitted as: Mark-Jan van der Meulen, Paul Steen, Hans Reinten, Herman Wijshoff, Michel Versluis and Detlef Lohse, Non-axisymmetric effects in drop-on-demand piezo-acoustic inkjet printing. 
it is needed and that no mask is required. This promises a reduction in production time, cost and waste for a large range of other applications [5]. Well-known fields in which DoD inkjet printing is applied are printed electronics [47, 48], organic light emitting diodes (OLEDS) [49] and organic solar cells [50]. The digital nature of the technique is interesting because on demand personalized products can be produced. Applications fields that benefit from this property include integrated smart systems, e.g. RFID tags [51], and the highly popular 3D rapid prototyping [52]. The noncontact nature of inkjet-printing allows not only applications on flat surfaces but on more arbitrary shaped surfaces aswell, which makes it suitable for all kinds of decorating purposes and deposition on fluidic and other highly fragile structures [53].

The print resolution required for graphical inkjet products is bounded by the spatial resolving capacity of the eye (visual acuity), which for optimal eyes and lightning conditions have been found to be 77 cycles per degree [54]. Therefore the maximal observable resolution depends on the viewing distance; typically a $10 \times 15 \mathrm{~cm}^{2}$ photograph is observed from a distance of $25 \mathrm{~cm}$, which demands an optimal resolution of $\approx 900 \mathrm{dpi}$ (dot per inch). Full color prints require multiple dots per pixel, usually 4 for CMYK-prints. which makes the typically found 600 ppi (pixel per inch) acceptable. For many of the upcoming applications in functional printing, however, a much higher resolution is required. Sub-picoliter drops are required to obtain printed features finer than 2400 dpi resolution, corresponding to $10 \mu \mathrm{m}$ features. Current commercial 3D printing resolutions are $42 \mu \mathrm{m}$ in the lateral direction and $16 \mu \mathrm{m}$ in height and the resolution has to be further improved to expand on the number of possible applications [2]. Inkjet technology is interesting for the production of multiple organic electronic devices, but for the use in organic thin film transistors (OTFTs) a further reduction of the scale is required, since the currently obtained $10 \mu \mathrm{m}$ features limit the operational throughput of these devices [55]. Also for maskless lithography production methods, the size of the droplets has to be reduced by at least another order of magnitude [56].

The use of microelectromechanical systems (MEMS) technology allows for a reduction of the nozzle sizes. For MEMS print head manufacturing accuracy in the $0.5 \mu \mathrm{m}$ range has been reported $[57,58]$. In the graphical industry, nozzle tolerances of $<0.1 \mu \mathrm{m}$ are pursued. The size of the droplets formed, in principle, is comparable to the nozzle size. However, the droplet size can be reduced through the use of actuation pulse modulation [59,60]. Here, higher order modes of the meniscus oscillations are used to eject droplets that are one order of magnitude smaller than the typical nozzle size. Also, pulsed DC voltages have been applied in combination with electrohydrodynamic inkjet printing [61], leading to controlled drop formation with a drop-to-nozzle diameter ratio down to 1:15.

While the resolution of a printed product is primarily determined by the droplet size, after impact and solidification, it is also determined by the droplet deposition 


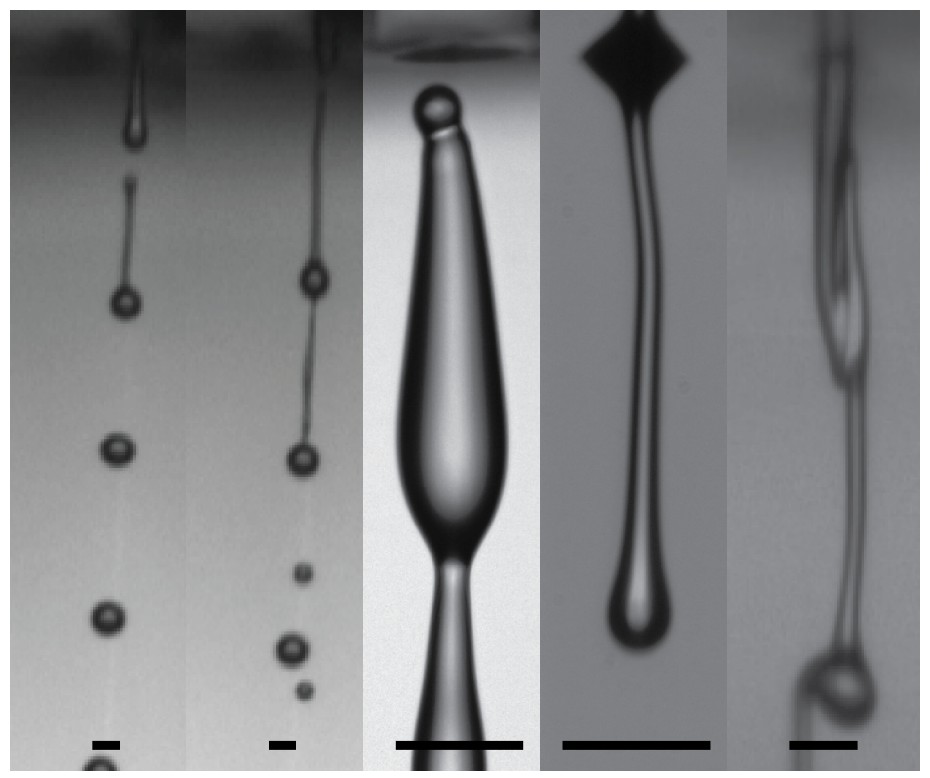

(a)

(b)

(c)

(d)

(e)
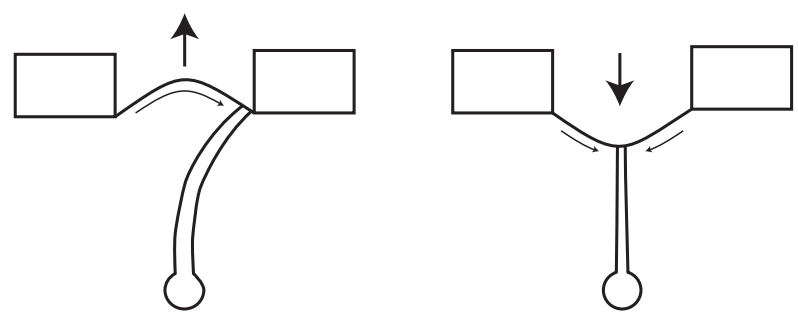

(f)

Figure 3.1: Observed asymmetrical drop formation in different print heads. The black lines indicate $50 \mu \mathrm{m}$. (a) Jet angle: drops are jetted away from the central axis of the nozzle. (b) Satellite formation: satellite drops are easily jetted away from the main drop. (c) Tail hooking: the tail drop is pulled away towards the nozzle edge. (d) Croocked tail: the droplet tail is pulled to the nozzle wall and pushed back to the center axis causing a deflection in the tail. (e) Sidewards pushed drops: when a droplet is jetted shortly after an asymmetrical drop, the new drop is strongly affected. (f) Tail hooking, the tail attached to the meniscus has a preference to sweep to one side of the nozzle upon retraction, and is centered during extortion of the meniscus outside of the nozzle. 
accuracy [62]. For example, display applications for smart phones require an placement accuracy of approximately $5 \mu \mathrm{m}$ [63]. The deposition accuracy depends on: the accuracy of the movement of the print head with respect to the paper, the aerodynamic effects on the droplet in flight, the directional accuracy of jetting, and the drop formation precision and reproducibility. It has been found, both numerically and experimentally, that the aerodynamic effects of moving paper has a negligible influence on the droplet displacement of $20 \mu \mathrm{m}$ droplets [64, 65]. However, smaller drops become increasingly more vulnerable to the surrounding air flow. In addition, the smallest droplets, i.e. the emerging satellite drops, do not carry enough momentum to reach the substrate and can agglomerate on the nozzle plate, which may lead to nozzle failure. And finally, to maintain the material flow throughput whilst reducing the droplet size, the DoD frequency must be increased, which unfortunately gives less reproducible drop formation [66].

The deposition accuracy of jetted droplets is strongly affected by non-axisymmetric drop formation. Figure 3.1 illustrates some of the well known examples of nonaxisymmetric effects in droplet formation. Figure 3.1(a) displays a problem with jetting directionality also referred to as jet angle, where the droplet is jetted at an angle with respect to the direction parallel to the nozzle plate. These are problematic for inkjet devices since the droplet is not deposited at the target site on the substrate. Only constant jet angle in the direction of the motion of the print head, can be corrected for using additional time delays. But often this is not the case and the jet angle also changes during the jetting process which gives rise to large deposition inaccuracies $[67,68]$. Asymmetrical drop formation, such as those shown in Fig. 3.1(b) gives rise to an increased generation of satellite drops [21]. Satellite drops are typically formed from the Rayleigh-Plateau breakup of thin droplet tails. In a axisymmetrical situation, the trailing satellites can easily merge with the following drops due to their different velocities. However, for the non-axisymmetrical case, the satellites can be directed along a different trajectory than the main drops, reducing their chance to be captured. Upon retraction of the meniscus into the nozzle, the connected droplet tail is often observed to sweep to one side of the nozzle, making the subsequent drop formation process non-axisymmetric, see Fig. 3.1(c). If the tail of the droplet is not yet pinched off from the meniscus at this stage, the deflection will cause so-called tailhooking $[24,28]$. This process can be compared to a string under tension, confined at one side to a spherical cap. By sweeping the tail to one side, it will shorten and release its stress, as indicated in Fig. 3.1(f). In a later stage, where the meniscus is outside the nozzle, the preferred position of the tail will be at the apex of the meniscus. Thus, the particular orientation of the droplet tail will depend on the exact moment of pinch-off. When the tail pinches off from the meniscus at the moment when the meniscus retracts, the tail droplet and possible satellite droplets are catapulted in a sideways direction. In case the tail droplet does not detach from the meniscus, it 
restores to the center position resulting in a crooked tail, see Fig. 3.1(d). The main droplet is not strongly affected by the occurrence of an asymmetry in the tail, due to the large difference in inertia. However even jet angles down to $0.1^{\circ}$ cause a relevant and measurable change in drop deposition position. At higher DoD frequencies, the droplet is not easily pinched off from the meniscus, before the following droplet is produced. When the first droplet is not perfectly symmetric, the next droplet will flow alongside it, enhancing the asymmetry. This can give rise to a zig-zag drop formation mode with higher-order or chaotic deviations from the symmetric drop formation, see Fig. 3.1(e).

The asymmetries in real inkjet devices are often initiated by asymmetrical effects near the nozzle and the origin of these effects are suspected to trigger the deviation of jetting with respect to the nozzle axis, see the set of illustrations in Fig. 3.2. A important problem are asymmetries in the print head and nozzle geometry, Fig 3.2(a). These include positional or rotational misalignments of the print head channel with respect to the nozzle. Moreover, dirt or dust particle agglomerates stuck in the nozzle area can form an obstacle that redirects the fluid flow, see Fig. 3.2(b). One further problem is a wetted nozzle plate typically is not perfectly symmetric around the nozzle entrance. Therefore the meniscus will not be fully pinned to the nozzle edge but at some point may coalesce with the wetted area around the nozzle. Thus, the asymmetry initiated by the location of the wetted area will then result in an asymmetric ejection from the nozzle, see Fig. 3.2(c). Finally, bubbles entrapped inside the inkjet nozzle can grow due to rectified diffusion $[69,70]$. When these bubbles become too large, see e.g. Fig. 3.2(d) they can absorb the applied pressure wave which will lead to nozzle failure [41]. However, even a smaller bubble, one that can freely move within the nozzle, may cause an asymmetric effect to the pressure wave directed towards the meniscus.

In the last few years there has been a growing interest in non-axisymmetrical drop formation. Kwon et al. [71] reported on the axi-switching of a drop asymmetry in time resulting from an elliptical nozzle. Castrejón-Pita et al. [72] showed that mechanical defects outside the nozzle plate have a negligible effect on the drop formation, but when the defect intrudes into the nozzle flow strong deflection angles are introduced. Very recently Harlen et al. [24] showed experimentally and numerically that droplets detach asymmetrically from nozzles which are under an angle with respect to the nozzle plate. While the non-axisymmetric drop formation in the above work has a clear geometrical origin, which in several cases was intentionally imposed by the authors, it can also be hypothesized that non-axisymmetric drop formation can be the result of unstable asymmetrical surface modes at the meniscus interface. The physical mechanisms that are known to drive meniscus instabilities are the RayleighTaylor instability [73, 74] and the parametrically driven instability [75]. Both give rise to symmetric and anti-symmetric surface modes. To understand the surface mode 
Positional misalignment

Directional misalignment

Channel/
Funnel
Nozzle plate

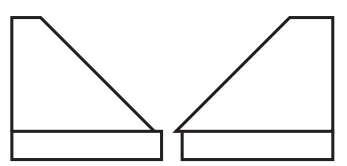

Channel

Nozzle plate

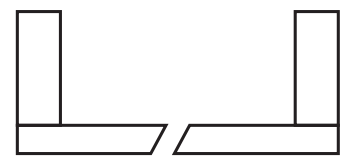

(a)

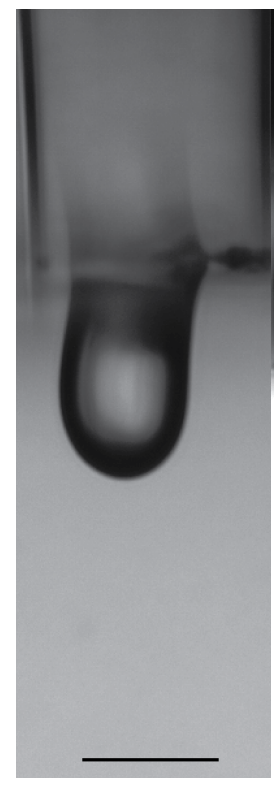

(b)

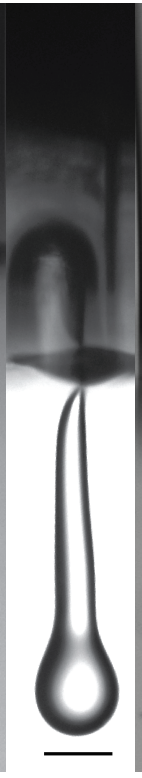

(c)

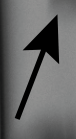

C

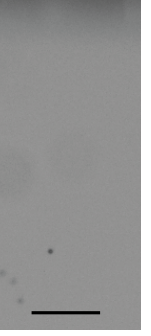

(d)

Figure 3.2: Asymmetric drop formation by geometrical origin: (a) Print head and nozzle geometry asymmetries. (b) Dirt in the nozzle, causing the drop to deflect. (c) Asymmetrically wetted nozzle plate easily results in coalescence of the tail drop with the wetted area and deflection of the droplet. (d) Bubble entrapped off-center inside the nozzle, indicated with in arrow, deforming the acoustic actuation wave asymmetrically. The black lines indicate $50 \mu \mathrm{m}$. 
instability of the meniscus we study the system numerically within the given acoustic and fluidic parameter space. We find approximate acceleration thresholds for the symmetric and asymmetric surface modes for both of the instabilities. We then experimentally extract the acceleration of the meniscus interface in a transparent inkjet system to compare the obtained values with the numerical results.

\subsubsection{Print head}

The AD-K-501 Autodrop Pipette of Microdrop technologies is built from a single nozzle glass tapered nozzle and is actuated with a cylindrical piezo element. The $25 \mu \mathrm{m}$ radius nozzle is connected to a long tapered channel, therefore the resonance frequency of this print head is predicted by the wave guide theory [76] and is determined by the traveling time of the pressure wave through the microchannel. This type of print head is typically used for water-based inks. From the oscillation frequency of the meniscus, the resonance frequency of this print head was estimated to be $7 \mathrm{kHz}$. The glass nozzle is optically transparent and the meniscus movement within the nozzle can be traced. In the following we will refer to this print head as the 'Microdrop'.

\subsection{Theoretical approach to the meniscus instability}

In this section we treat the Rayleigh-Taylor instability and the parametrically driven instability. For each of these instabilities, we find the accelerations for which the interface becomes unstable and their typical growth times, which is depended the wavenumber of the perturbations. We evaluate these acceleration thresholds and growth times for the Microdrop print head. In appendix 3.A, the evaluation of a typical high frequency print head with a more viscous fluid is given.

\subsubsection{Rayleigh-Taylor instability}

Accelerating two fluids with different density into one another causes surface instabilities when the inertial forces overcome the restoring surface tension. For the inkjet situation, we consider the ink-air interface. In general we consider the stability of surface of fluid 2 on top of fluid 1 with densities $\rho_{2}$ and $\rho_{1}$, see Fig. 3.3.

Viscosity can be neglected for a small Ohnesorge number: Oh $=\mu_{L} / \sqrt{\rho_{L} \sigma R} \ll 1$, with $\mu_{L}, \rho_{L}$ and $\sigma$ the viscosity, density and surface tension of the liquid and $R$ the radius of the nozzle. We find for the Microdrop print head and water $\mathrm{Oh}=0.023$. Thus, in the water-based system, the viscosity is of minor importance.

For small Oh a perturbation of the surface can be described with potential flow. In cylindrical coordinates the general solution for the deflection amplitude $\eta(t)$ of 


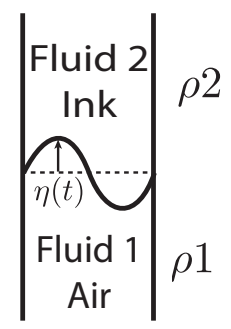

Figure 3.3: Rayleigh Taylor instability

the mensicus[77] reads:

$$
\eta(t)=\varepsilon J_{m}\left(k_{m n} r\right) e^{i(m \theta+\omega t)}
$$

where $J_{m}$ is the Bessel function of the first kind and $k_{m n}$ the wavenumbers of the perturbations. Given the nozzle radius, these wavenumbers are selected through the boundary condition. For a pinned meniscus at the nozzle edge or wall, $J_{m}\left(k_{m n} R\right)=0$ with $R$ the radius of the nozzle. A commonly used boundary condition, however, is a moving contact line with a constant contact angle of $90^{\circ}$, which gives: $J_{m}^{\prime}\left(k_{m n} R\right)=0$. The allowed wavenumbers correspond to symmetric and asymmetric meniscus eigenmodes as illustrated in Fig. 3.4. The stability in time of the deflection is determined by whether $\omega$ is real or not.

The non-viscous dispersion relation in free space [78] is given by:

$$
\omega^{2}=\frac{\left[\sigma k_{m, n}^{2}+a\left(\rho_{1}-\rho_{2}\right)\right] k_{m n}}{\rho_{2}+\rho_{1}},
$$

where $a$ is the acceleration of the interface and $\sigma$ the surface tension. From this equation it follows that the perturbation is always stable if $\rho_{2}<\rho_{1}$ and is unstable for the allowed combinations $k_{m, n} R$ when the Bond number

$$
\mathrm{Bo}=\frac{a R^{2}(\Delta \rho)}{\sigma}>\left(k_{m, n} R\right)^{2} .
$$

The combinations of $k_{m, n} R$ for the first growing modes when Bo is increased are also indicated in Fig. 3.4. It shows that the first unstable mode for a pinned meniscus is symmetrical and for the constant contact angle it is asymmetrical. The meniscus in an inkjet nozzle is subjected to typical accelerations of up to $10^{5}$ to $10^{7} \mathrm{~m} / \mathrm{s}^{2}$, which dominates strongly over the gravitational effect, therefore pressure-induced acceleration of the inkjet meniscus will be treated here rather than the gravitational acceleration.

Some comments of the approximations used here are in order. The above solutions are treated as the standard RT problem exhibiting a flat interface. The excess 


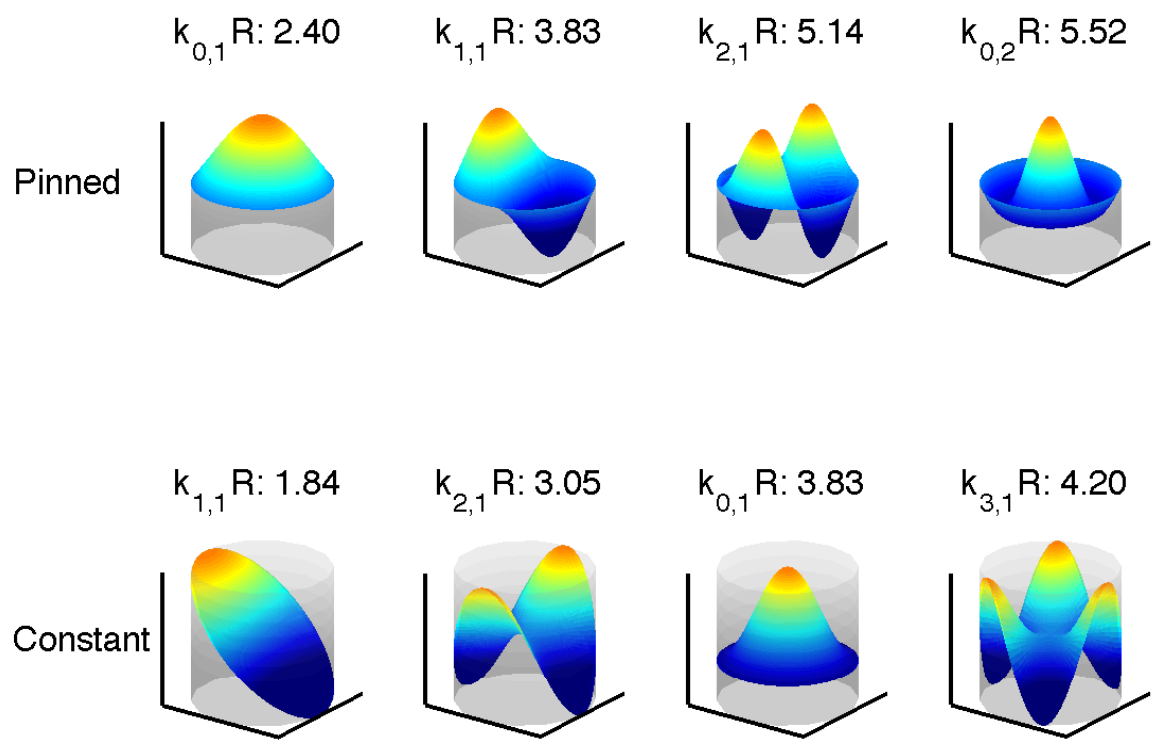

Figure 3.4: The eigenmodes of the meniscus shapes are selected by matching the solution of equation 3.1 by the boundary condition at the nozzle wall. The selected shapes for a pinned and for constant $90^{\circ}$ contact angle are plotted. The product of the wavenumber and the radius is set by the condition of the surface at the wall, and is indicated the eigenmodes.

pressure due to curvature of the interface has been treated by Achard et al. [79]. They show theoretically that for curved surfaces there also exists a range of steady solutions for $\mathrm{Bo}>\left(k_{m n} R\right)^{2}$. The inclusion of viscosity is known to induce a small frequency shift [78] $\omega=\omega_{0}(1-\delta)$ with $\delta$ the non-dimensional damping and an increase in the growth time of the perturbations. Chandrasekhar [78] found that the inclusion of viscosity for an infinite surface does not change the critical wavelengths, but that it indeed decreases the growth rate of the disturbances. Here the appearing wavenumbers are selected by the nozzle size and we assume that the effect of viscosity will only have an effect on the growth time of the perturbation.

An approximation for the growth time of the different wavenumbers can be directly obtained from Eq. 3.2. When all the wavenumbers are present in a system without walls, the fastest growing wavenumber is the one that is observed. In the bounded system, however, only wavenumbers are selected which fit the nozzle, and the fastest growing selected mode will dominate. In Figure 3.5 the growth times are 


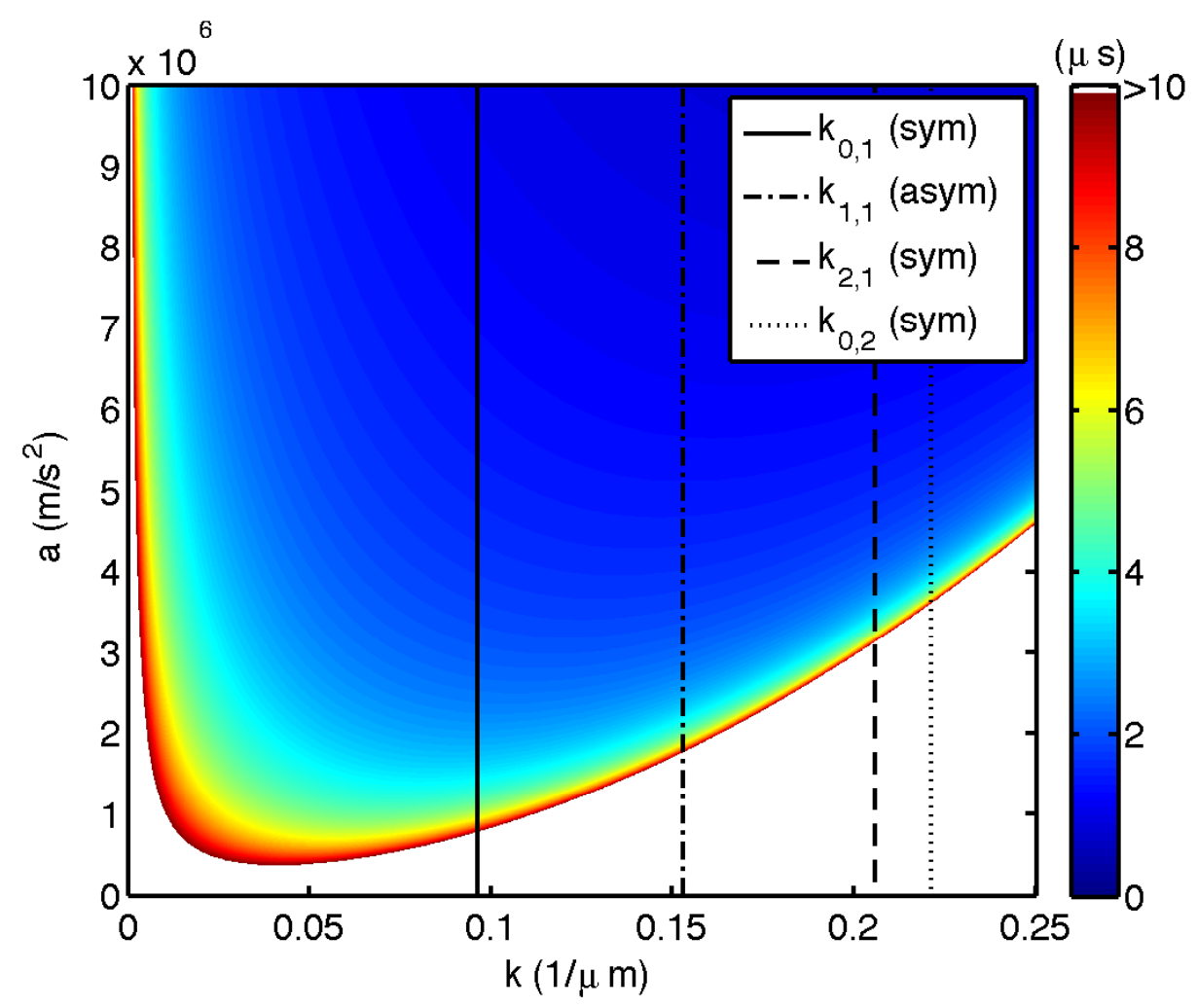

Figure 3.5: The growth time of perturbations at the interface for accelerations of the fluid and wavenumber of the perturbation. A water interface in a nozzle with a radius of $25 \mu \mathrm{m}$. The vertical lines indicate the first four unstable modes for the pinned meniscus.

plotted for the first four appearing wave modes for the Microdrop, with corresponding nozzle radii and fluid properties. The first growing mode for the pinned boundary condition is axi-symmetric and grows for accelerations larger than $7.7 \times 10^{5} \mathrm{~m} / \mathrm{s}^{2}$. For an acceleration $a=10^{6} \mathrm{~m} / \mathrm{s}^{2}$ in the Microdrop nozzle, the only unstable mode is $k_{1,1}$, but when the acceleration is further increased to $5 \times 10^{7} \mathrm{~m} / \mathrm{s}^{2}$ all four modes are present and all are approximately equally fast growing.

\subsubsection{Parametrically driven capillary instabilities}

A parametric forcing of $a \cos (\omega t)$ normal to the meniscus pumps most energy in alternating harmonic $(\omega, 2 \omega, 3 \omega$, etc. $)$ and subharmonic modes with frequencies at $\omega_{0}=\omega / 2,3 \omega / 2$, etc. The inviscid solution gives normal modes [80] $\eta(t)=$ $\varepsilon_{k m}(t) J_{m}\left(k_{m n} r\right) e^{i(m \theta)}$. The mode shapes are the same as those depicted in Fig. 3.4. 
However the mode amplitude $\varepsilon_{k m}(t)$ and stability depend on the driving amplitude and frequency. The non-viscous growth of the disturbances follows the classical Mathieu equation [81], describing parametrically driven oscillations. For small damping (i.e. $\left.v /\left(\lambda^{2} \omega\right) \ll 1\right)$ flow can still be considered to be irrotational except for a small layer around the interface. We find $5.3 \times 10^{-3}$ for the Microdrop. The perturbation $\eta$ can then be approximated by adding a phenomenological damping term to the Mathieu equation [82]. Although this equation is only accurate in first order in $v k^{2} / \omega$ [83], the solution gives insight in the stability behavior. The damped Matthieu equation reads:

$$
\eta^{\prime \prime}+2 d \eta^{\prime}+\omega_{0}^{2}(1-\hat{a} \cos (\omega t)) \eta=0
$$

with the natural frequency $\omega_{0}$, which reduced due to the small nozzle to the capillary dispersion relation:

$$
\omega_{0}^{2}=\frac{\left(\rho_{1}-\rho_{2}\right) g k+\sigma k^{3}}{\rho_{1}+\rho_{2}} \rightarrow \frac{\sigma k^{3}}{\rho_{1}+\rho_{2}}
$$

the dimensionless forcing acceleration

$$
\hat{a}=\frac{a k\left(\rho_{1}-\rho_{2}\right)}{\omega_{0}^{2}\left(\rho_{1}+\rho_{2}\right)},
$$

and the viscous damping coefficient

$$
d=2 k^{2} \frac{\mu_{1}+\mu_{2}}{\rho_{1}+\rho_{2}} .
$$

The latter term is the viscous dissipation of the bulk fluid, to first order in $v k^{2} / \omega$, and becomes inaccurate for higher viscosities. To include dissipation effects at the wall or at the interface slightly different damping coefficients have been proposed [77].

The stability analysis of the Matthieu equation results in a plot of tongues of alternating subharmonic and harmonic responses to the driving. For finite viscosity these responses need a critical acceleration to grow. We must stress at this point that the resonance frequency of the print head, and thus the movement of the meniscus as denoted in section $3.1 .1(\mathrm{f}=7 \mathrm{kHz} / \omega=44 \mathrm{kHz})$, is lower than the natural frequency of the surface $(\omega=270 \mathrm{kHz})$, given by Eq. 3.5. This results in a much weaker coupling of the actuation energy into surface waves. Figure 3.6 shows the stability boundaries for water driven at $\mathrm{f}=7 \mathrm{kHz}$. The boundaries are found using the Floquet theorem, following Kumar et al. [84]. The colors in the figure indicate the calculated growth times of the instabilities from solutions of the Matthieu equation. The subharmonic modes are growing faster than the harmonic modes, as expected, due to the lower onset at lower accelerations. Although the first subharmonic is the one that grows fastest for the smallest acceleration, in the bounded case the fifth harmonic is selected 


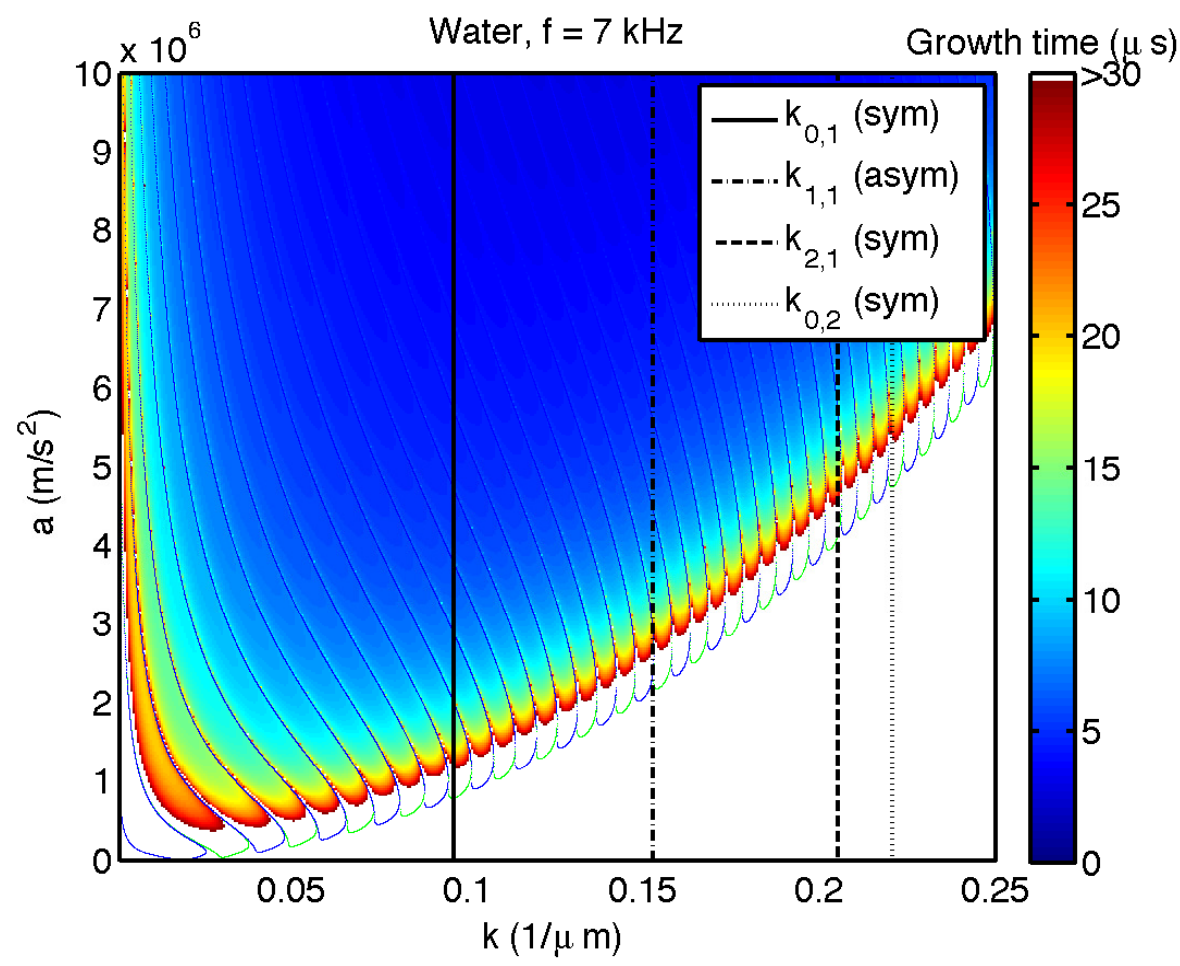

Figure 3.6: Acceleration threshold for parametrically driven instability. A. Water at $7 \mathrm{kHz}$ driving, the vertical lines indicate the selected wavenumbers for $R=25 \mu \mathrm{m}$. The first subharmonic tongue has the lowest critical acceleration, however the first wavenumber that fits the nozzle selects a higher harmonic.The colors indicate the calculated growth time of the instability.

by the $k_{0,1}$ mode, the selected wavenumbers for a pinned meniscus being indicated by the vertical lines in Fig. 3.6(a).

The critical accelerations for the selected wavenumbers are found and presented in Table 3.1. We find that these acceleration thresholds are similar to or higher than the thresholds for the same modes in the Rayleigh-Taylor analysis.

\subsubsection{Towards experimental validation}

From the analysis of the RT and parametric instability we find the following system variables: the print head variable $R$, the fluid variables $\rho, \sigma$ and $\mu$ and finally the driving variables $\omega$ and $a$. The frequency $\omega$ in standard DoD inkjet printing is the main frequency of the actuation pulse, which is in optimal driving conditions the resonance frequency of the print head. The acceleration of the meniscus can be 


\begin{tabular}{|c|c|c|c|c|}
\hline Pinning condition & Wave mode & $\mathrm{k} * \mathrm{R}$ & $\mathrm{k}_{R=25 \mu \mathrm{m}}(1 / \mathrm{m})$ & $a_{c}\left(10^{6} \mathrm{~m} / \mathrm{s}^{2}\right)$ \\
\hline \multirow{4}{*}{ Pinned meniscus } & $k_{0,1}$ & 2.4 & $0.96^{*} 10^{5}$ & 0.8 \\
& $k_{1,1}$ & 3.83 & $1.53^{*} 10^{5}$ & 2.2 \\
& $k_{2,1}$ & 5.14 & $2.06^{*} 10^{5}$ & 4.2 \\
& $k_{0,2}$ & 5.52 & $2.21 * 10^{5}$ & 4.7 \\
\hline \multirow{4}{*}{ Constant contact angles } & $k_{1,1}$ & 1.84 & $0.74^{*} 10^{5}$ & 0.5 \\
& $k_{2,1}$ & 3.05 & $1.22^{*} 10^{5}$ & 1.3 \\
& $k_{0,1}$ & 3.83 & $1.53 * 10^{5}$ & 2.2 \\
& $k_{0,2}$ & 4.20 & $1.68^{*} 10^{5}$ & 2.9 \\
\hline
\end{tabular}

Table 3.1: Critical acceleration of selected wavenumbers for the at $7 \mathrm{kHz}$ parametrically driven water meniscus in a $25 \mu \mathrm{m}$ nozzle.

controlled by changing the actuation voltage of the piezo acoustic print heads. The acceleration of the meniscus can be found by tracing the meniscus positions from high-speed recordings. An optical transparent nozzle makes it possible to trace the meniscus both outside and inside of the nozzle. From these experiments we can deduce whether the stability thresholds for the Rayleigh-Taylor or parametrically driven instability are crossed during jetting.

\subsection{Experimental investigation}

\subsubsection{Visualization setup}

For reproducible drop formation time-resolved single flash illumination can be used to obtain high-resolution stroboscopic information on the drop formation [85]. The droplets are recorded in transmit bright-field illumination mode, see Fig. 3.7. For every image recording a fresh droplet was produced while an incremental delay of the recording with respect to the drop actuation was imposed. The ejected microdroplets typically move with a velocity of $10 \mathrm{~m} / \mathrm{s}$, therefore, the illumination time has to be set smaller than $30 \mathrm{~ns}$ for a typical resolution of $300 \mathrm{~nm} /$ pixel to prevent motion blur. The illumination was provided by a Nd-YAG laser pulse (pulse length of $7 \mathrm{~ns}$ at a wavelength of $532 \mathrm{~nm}$ ) and a fluorescent diffuser (LaVision, type number) making use of the iLIF technique [10].

\subsubsection{Analysis of meniscus movement from recorded image sequences.}

Most of the meniscus movement takes place inside of the nozzle, therefore the acceleration of the meniscus were evaluated inside the nozzle, by observation through the 


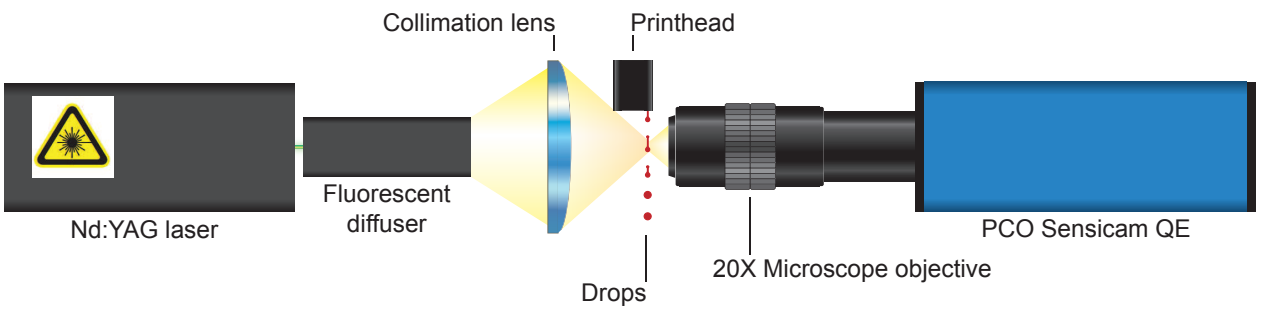

Figure 3.7: Experimental setup: A $7 \mathrm{~ns} \mathrm{Nd}$ :YAG laser pulse is converted to noncoherent fluorescence light for illumination by a fluorescent diffuser. Stroboscopic imaging with incremental delays with respect to the actuation pulse make up a full recording of the meniscus movement.

transparent nozzle. The position of the meniscus is determined by finding the inflection point of the intensity profile across the fluid boundary. Figure 3.8 shows typical traces of the meniscus during an actuation cycle, recorded with $0.1 \mu$ s increasing delay time steps. The meniscus first is pulled back into the nozzle, where it reaches its deepest position around $t=45 \mu \mathrm{s}$. At around $t=70 \mu \mathrm{s}$ the meniscus reaches the nozzle exit after which the droplet is formed. And finally at $t=140 \mu$ s the meniscus retracts back into the nozzle. Then the cycle repeats with a period of oscillation of approximately $175 \mu \mathrm{s}$. On top of this global movement a higher frequency meniscus movement is observed, and are observed as dents and bumps in the position trace.

The acceleration is extracted from the meniscus position. The experimental noise, however, does not allow for the calculation of the second derivative of the positiontime data directly and the data is smoothed using low-pass frequency filtering. The time steps of the time resolved series are $0.1 \mu \mathrm{s}$, giving noise in the $\mathrm{MHz}$ range, the meniscus moves typically with order $10 \mathrm{kHz}$, and a more rapid oscillation is observed on top of the main meniscus movement with a typical frequencyorder of $100 \mathrm{kHz}$. Applying low pass filters with a cutoff frequency $40 \mathrm{kHz}$ and $140 \mathrm{kHz}$ gave optimal results while maintaining the separation of scales of the acceleration of the meniscus. 


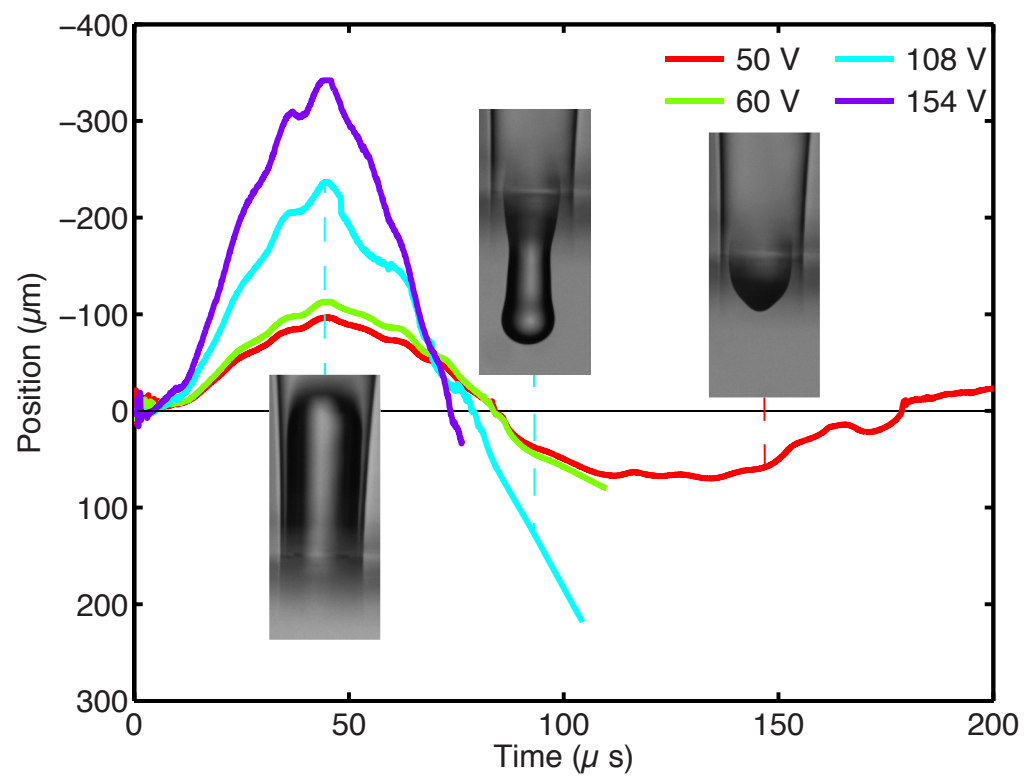

Figure 3.8: Meniscus traces for different actuations voltages of the Microdrop print head, see Section 3.1.1. In the figure the apex positions are shown at the corresponding position in the time. A negative value indicates a position inside the nozzle. For the $50 \mathrm{~V}$ actuation the meniscus oscillates within the nozzle. For actuations of $60 \mathrm{~V}$ and higher a droplet is ejected.

\subsection{Results}

We now describe the results from the Microdrop dispenser with water and a nozzle radius of $25 \mu \mathrm{m}$. Driving conditions have been plotted in Fig. 3.8 for experiments with driving voltages of 50,60, 108 and $154 \mathrm{~V}$. The first driving voltage did not create sufficient pressure to form a droplet, but instead the meniscus was oscillating around the nozzle exit. For a $60 \mathrm{~V}$ driving pulse a droplet was formed, but the velocity of the resulting droplet was very low $(<0.2 \mathrm{~m} / \mathrm{s})$. For the higher voltages droplets were ejected, see again Fig. 3.8.

The main meniscus movement is plotted in Fig. 3.9. In the lower part of the figure the acceleration is plotted together with the expected instability thresholds for the instabilities. The thresholds for the constant contact angle are indicated with the symbol (c). For the actuation with voltages $108 \mathrm{~V}$ and higher the acceleration thresholds for the axisymmetric $k_{0,1}$ are exceeded when the meniscus accelerated inwards of the nozzle, around $8 \mu \mathrm{s}$ after the actuation of the print head, and when it is accelerated back outwards of the nozzle at time $40 \mu \mathrm{s}$. The RT instability sets in when the fluid with higher density is accelerated into the fluid with lower density, i.e. when 
the meniscus is pushed from retraction to out of the nozzle. The acceleration crossed the threshold for longer than $15 \mu \mathrm{s}$, which exceeds the typical growth time of the RT instability for $a \sim 10^{6} \mathrm{~m} / \mathrm{s}^{2}$, as indicated in Fig. 3.5. For the pinned meniscus the accelerations from the main meniscus movement never cross the acceleration thresholds for the asymmetrical modes. For some lower actuations, even when drops are ejected, none of the pinned thresholds are exceeded during actuation of the print head. For the non-pinned meniscus the first instability threshold is for the asymmetrical mode $k_{1,1}(c)$ at $a=0.38 \mathrm{~m} / \mathrm{s}^{2}$, which is crossed for all droplet actuations and close to the actuation acceleration were a droplet was just formed.

In Fig. 3.10, the same data are shown, but now with low-pass filtering at $140 \mathrm{kHz}$, keeping the higher oscillations. The accelerations involved with these oscillations are approximately four times higher and for most accelerations the $k_{0,1}$ thresholds are crossed. Also the $50 \mathrm{~V}$ actuation which did not eject a droplet exceeded this threshold. The time spans during which the accelerations are above the threshold are now shorter, 5-6 $\mu \mathrm{s}$. This is comparable with the expected growth times of the RT and parametrically driven instabilities, as indicated in Figs. 3.5 and 3.6. Furthermore it should be noted that for low actuation voltages the meniscus shape resembles the meniscus mode $k_{0,1}$ of Fig. 3.4. For actuation voltages larger than $90 \mathrm{~V}$, however, the observed meniscus shape resembles the mode $k_{0,2}$, where a protrusion in the centre area of the meniscus oscillates with a higher frequency around its rim as shown in Fig. 3.11. When the actuation voltage exceeds $110 \mathrm{~V}$ a smaller droplet will be ejected from this central area, even though the global meniscus movement is still at the $7 \mathrm{kHz}$ resonance frequency. 


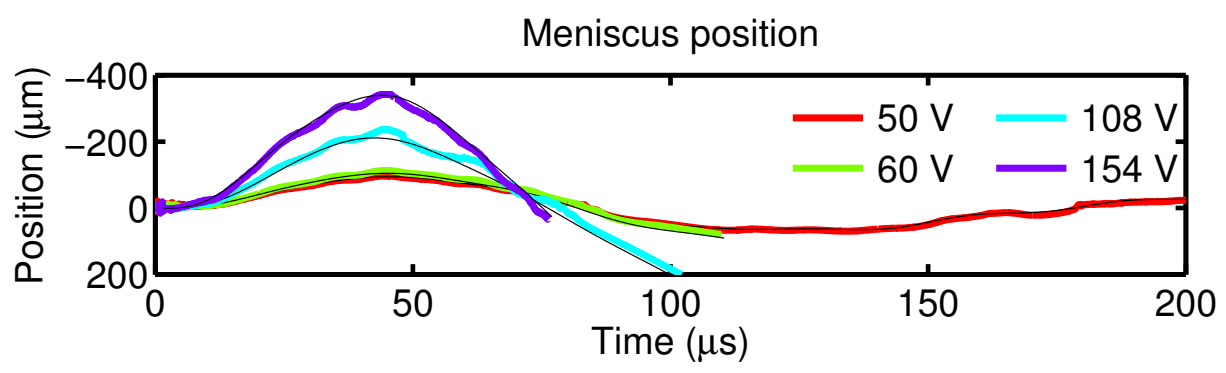

Critial accelerations

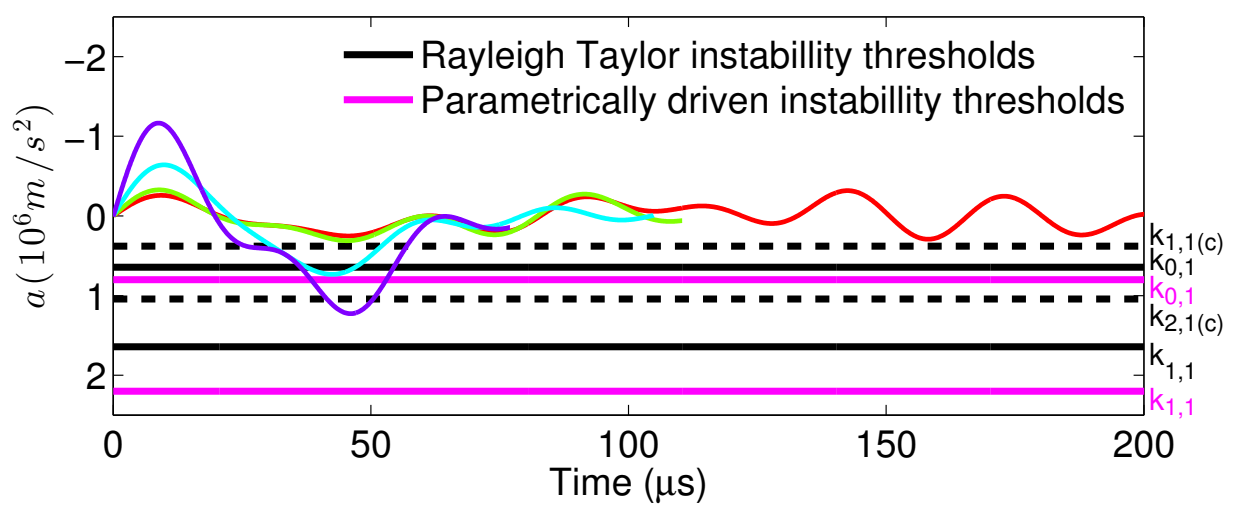

Figure 3.9: Thresholds for RT and parametrically forced instabilities for a pinned meniscus (solid lines) and contant contact angle (dashed lines/(c)). The acceleration is filtered with a $40 \mathrm{kHz}$ low-pass filter, which preserves the main meniscus movement, while filters out higher frequencies. The reconstructed meniscus position from the filter accelerations is plotted in black. The filtered accelerations are plotted in the lower figure with the instability thresholds. 


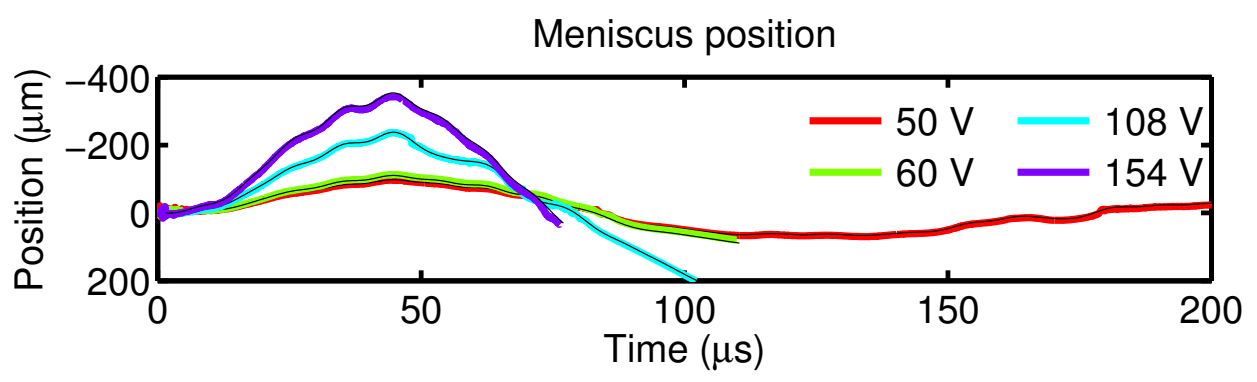

Critial accelerations

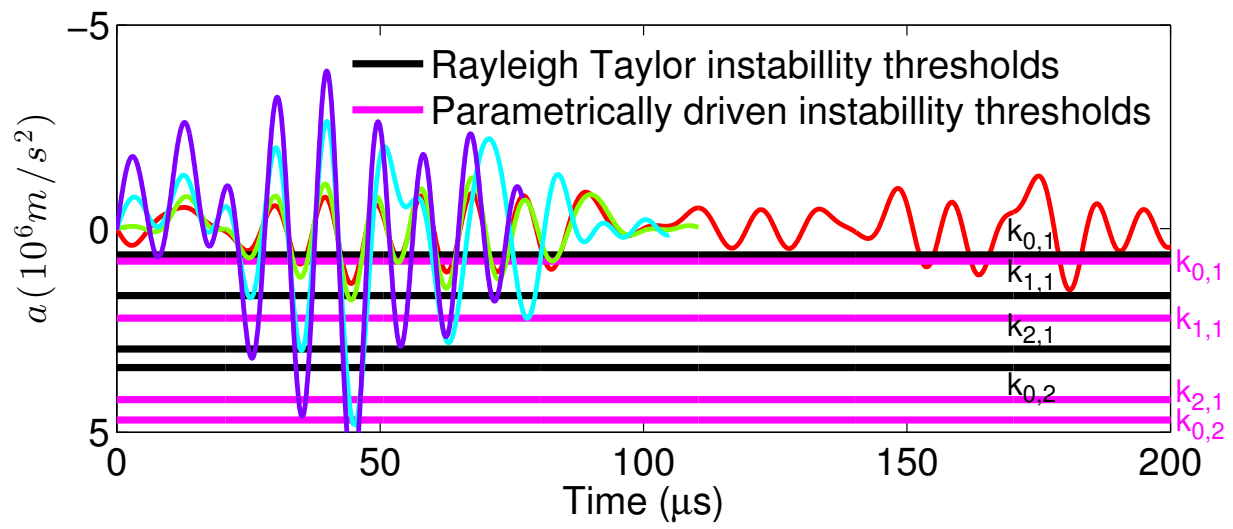

Figure 3.10: Thresholds for RT and parametrically forced instabilities. For clarity only the pinned meniscus thresholds are indicated. The acceleration is filtered with a $120 \mathrm{kHz}$ low-pass filter, preserving the main movement and higher oscillations of the meniscus. The reconstructed meniscus position from the filter accelerations is plotted in black. The filtered accelerations are plotted in the lower figure with the instability thresholds 


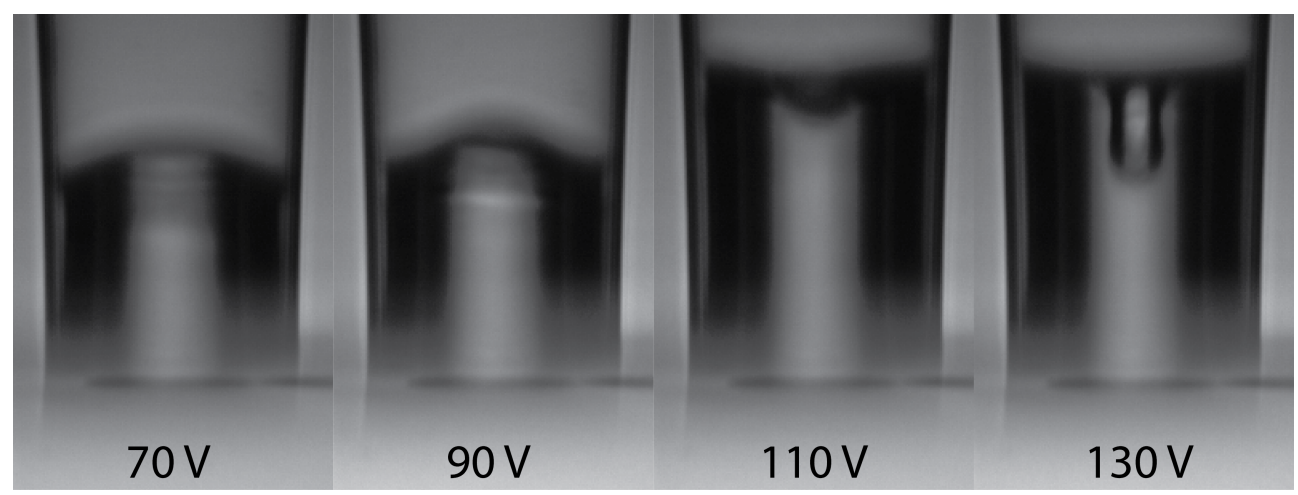

Figure 3.11: Experimentally observed meniscus shapes in the transparent nozzle. For lower actuation voltages the meniscus shapes resemble the symmetrical normal mode $k_{0,1}$, however for higher actuations the meniscus deforms to a shape resembling the (symmetrical) $k_{0,2}$ mode.

\subsection{Discussion}

This research has pursued to find out whether asymmetries in inkjet systems can be caused by unstable asymmetrical surface modes. Before we come to our final conclusions, we will address some remaining issues.

Viscosity has been treated in the theoretical aspects was found to have a small influence on the drop formation, such that the potential flow solutions including inertia and capillarity can be corrected with a viscous term. For a print head driven at low frequency with small viscosity fluids, where Oh is small, this is applicable. The parametrically driven instability solution is corrected with a viscous damping term, which is applicable when the effect of viscosity is constrained to the boundary layer. Due to the mismatch of the resonance frequency of the print head and the natural frequency of the meniscus for the parametric driven instability, we do not find lower acceleration thresholds with this analysis, but rather the resulting acceleration thresholds for instability are of the same order as in the Rayleigh Taylor instability analysis. Also the theoretical models are developed with the assumption that the initial interface is flat and a continuous acceleration acts on the surface. However, from direct optical observation the meniscus is hardly ever flat. When the first pressure wave reaches the meniscus it will bend to counteract the pressure. Also, it is common practice to initially shape the meniscus using negative pressure, as to curve it inwards, which excludes the flat meniscus assumption altogether.

For both instabilities investigated here the constant contact angles gives a lower instability threshold, which leads to the growth of the asymmetric sloshing mode $k_{1,1}$. From experimental observation the pinning boundary condition is often preferred, because it is easier to maintain than the $90^{\circ}$ contact angle condition. In the 
inkjet experiments performed, however, the pinning is not controlled. For small actuations the contact line is clearly stilled pinned at the nozzle plate, however in the experiments with stronger actuation, where the meniscus retracts far into the nozzle, it is not possible to distinguish between a pinned meniscus and a moving meniscus as can readily be observed from Fig. 3.11. Finally, in the experiments performed here a sloshing mode was never observed.

The theoretical thresholds were determined by balancing the acceleration force with the surface tension. In the experiments, however, we can only observe the effective acceleration which arises from the imbalance of the acceleration force and surface tension. Thus, the actual acceleration force is therefore underestimated by our experiments.

The Microdrop print head has a tapered nozzle, which means that the nozzle radius increases further down the nozzle, which decreases the acceleration threshold upon retraction of the contact angle. Finally, this print head also exhibits the tail hooking phenomenon as depicted in Fig. 3.1(c). Upon retraction of the meniscus in the nozzle the attached tail sweeps to one side of the nozzle, creating an asymmetrical feature in the drop formation. This phenomenon can be observed for very low driving situations, and correspondingly low accelerations. It was however always found in exactly the same position and orientation. Therefore is it is likely triggered by a geometrical asymmetry in the nozzle.

\subsection{Conclusions}

Asymmetric features in inkjet droplet formations have multiple origins, which can be caused by different known physical asymmetries in the inkjet system. The possibility for the growth of asymmetric surface modes has been investigated.

The acceleration threshold for the Rayleigh-Taylor and parametrically driven instabilities have been determined for a flat low-viscosity fluid meniscus in a cylindrical nozzle. We evaluated a $25 \mu \mathrm{m}$-radius nozzle Microdrop print head with water. The accelerations of the meniscus inside the optically transparent Microdrop during actuation were determined and compared to the calculated instability thresholds. It was found that the acceleration threshold for the first symmetrical mode can be exceeded during drop formation for both types of instability. The observed acceleration of the main meniscus oscillation, however, only exceeded the theoretical acceleration thresholds for asymmetrical modes for a non-pinned meniscus. On the other hand, it is observed that for larger accelerations a deformation of the meniscus develops, which grows and eventually ejects a smaller droplet. For those cases where the acceleration thresholds were exceeded the time that this acceleration was experienced by the meniscus was of comparable or longer duration compared to the theoretically determined growth times for both the instabilities. The higher frequency oscillations 
found superimposed on the meniscus have an even higher acceleration, and exceeds the calculated acceleration thresholds for even lower actuation voltages.

In the studied inkjet system asymmetric tail breakup has been observed. The fact that they can be recorded in a stroboscopic recording indicates that these asymmetries are perfectly reproducible and occur in the same position and orientation with respect to the nozzle. This suggests that these observed asymmetries are caused by a geometrically asymmetry in the nozzle. To analyze the pure Rayleigh-Taylor and parametrically driven instabilities, an optically transparent nozzle which jets entirely axisymmetrically would required. All our tested print heads with transparent nozzles, however, possessed some intrinsic asymmetry. To further investigate this problem, an upscaled system may be used, where the small geometrical defects in the nozzle have a less pronounced effect on the fluid movement.

\section{A High frequency print head}

In this section we evaluate the Rayleigh-Taylor and parametric instability for a high frequency print head. We use some typical numbers for a prototype print head of Océ Technologies B.V. in the Netherlands. This print head has a nozzle radius of $15 \mu \mathrm{m}$ which are etched in a silicon wafer, and is actuated with a piezo element. The nozzle is connected to a larger volume chamber, with the piezo actuator. Acoustically the high frequency print head can be treated as a Helmholtz resonator [76]. The resonance frequency of this print head is near $120 \mathrm{kHz}$ and therefore much higher DoD frequencies can be obtained as compared to the Microdrop system. The print head is used for high viscous inks such as hot-melt inks, which has viscosity: $10 \mathrm{mPa}$ s and surface tension: $30 \mathrm{~N} / \mathrm{m}$. This print head is not optically transparent for the visible light.

We find for the Ohnesorge number: $\mathrm{Oh}=0.47$, which indicates that the viscous effects for the Rayleigh Taylor problem are more important than for the water Microdrop case. And we expect to underestimate instability thresholds. We reproduce Fig. 3.5, for the high frequency print head.

Since the factor of $\frac{R^{2}}{\sigma}$ of equation 3.3 is approximately equal for the high frequency and Microdrop print head cases, the critical acceleration is also comparable for the selected wave numbers. The first growing mode for the pinned boundary condition is axi-symmetric and grows for accelerations larger than $6.7 \times 10^{5} \mathrm{~m} / \mathrm{s}^{2}$ for the prototype print head, were it was $7.7 \times 10^{5} \mathrm{~m} / \mathrm{s}^{2}$ for the Microdrop. The first growing mode for the $90^{\circ}$ contact angle meniscus is asymmetrical and grows for accelerations larger than $3.9 \times 10^{5} \mathrm{~m} / \mathrm{s}^{2}$. We find lower thresholds in this analysis, than for the Microdrop water case, since the surface tension of the hot melt ink is lower. However since the Ohnesorge number is larger, viscous dissipation should be taken into account to get better estimations of the thresholds. 


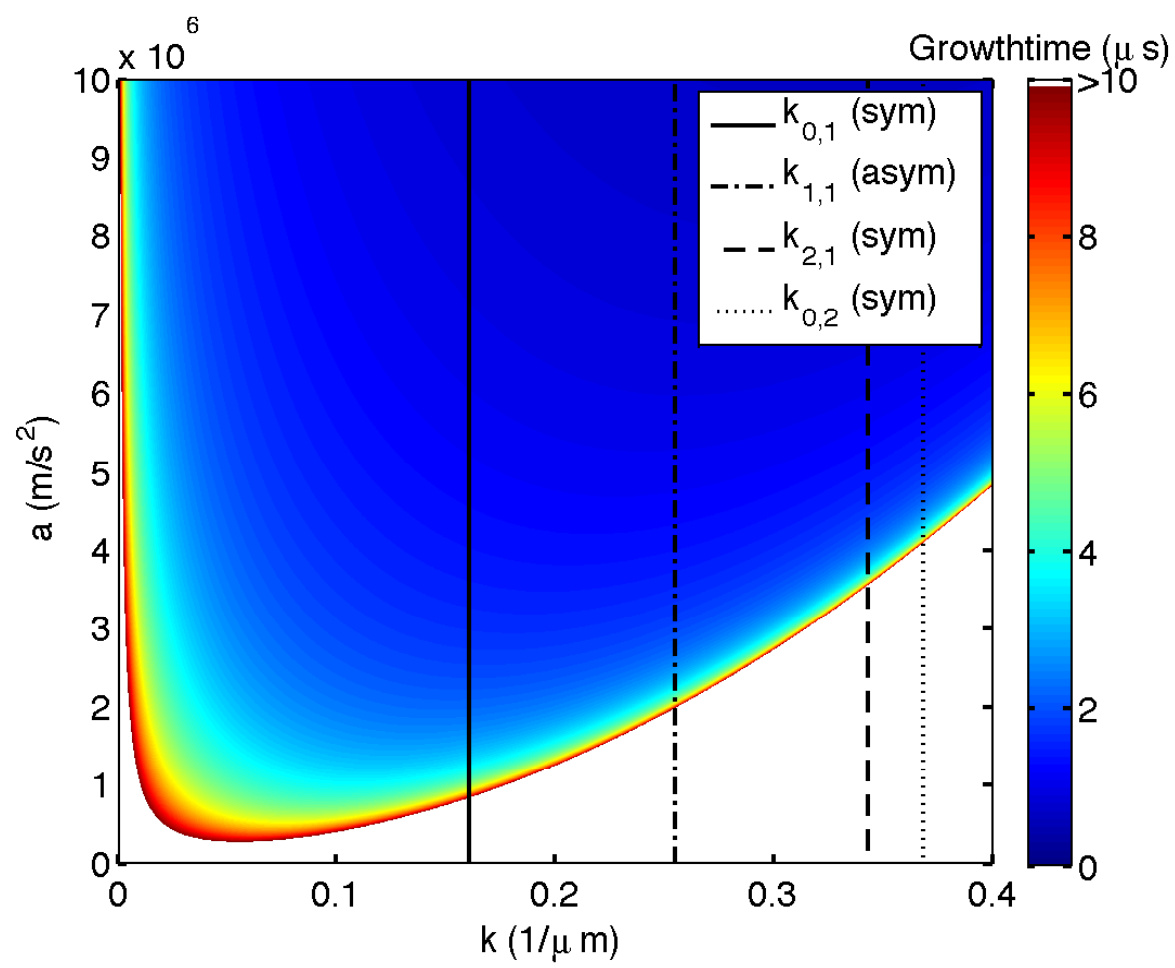

Figure 3.12: RT growth time of perturbations at the interface for accelerations of the fluid and wavenumber of the perturbation. An hotmelt interface in a $15 \mu \mathrm{m}$ radius nozzle. The vertical lines indicate the first four unstable modes for the pinned meniscus and appropriate nozzle size.

Now we also perform the analysis for the Parametric instability for in the prototype print head. For small damping condition (i.e. $v /\left(\lambda^{2} \omega\right) \ll 1$ ), we find 0.07 . We also reproduce Fig. 3.6, for the parametrically driven instability. For this print head the actuation frequency $f=120 \mathrm{kHz} / \omega=753 \mathrm{kHz}$ and the natural frequency of the surface $\omega_{0}=350 \mathrm{kHz}$.

Figure 3.13 shows the general behavior of increased thresholds due to a higher viscosity and the widening of the tongues due to the higher driving frequency. For this case we expect that the instability threshold for the $\mathrm{k}_{0,1}$ is much lower than for the other modes, indicated with the vertical lines. The modes $\mathrm{k}_{1,1}$ and higher require accelerations higher than $10 \times 10^{6} \mathrm{~m} / \mathrm{s}^{2}$. For the $90^{\circ}$ contact angle condition, the only selected mode is the asymmetric mode $\mathrm{k}_{1,1}$, for which holds: $R * k_{1,1}=1.84$, giving $\mathrm{k}_{1,1}=0.12$. This condition therefore results in a lower threshold than for the pinned meniscus condition. 


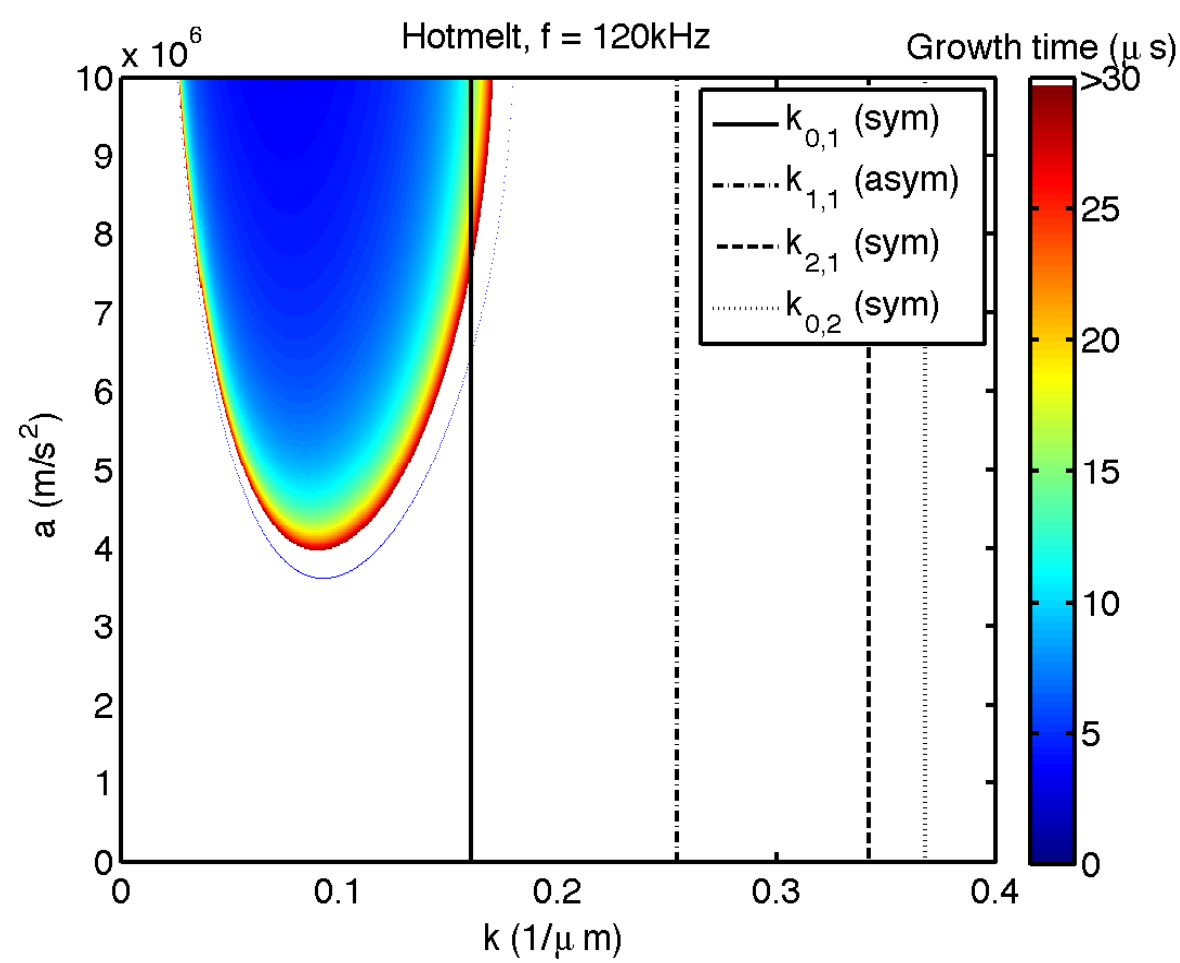

Figure 3.13: Acceleration threshold for parametrically driven instability. High frequency print head driven at $120 \mathrm{kHz}$. The vertical lines indicate the thresholds for the pinned meniscus. The higher viscosity raises the tongues and the higher driving frequency widens them. For this case only one mode is unstable for accelerations up to $10^{7} \mathrm{~m} / \mathrm{s}^{2}$. 


\section{4}

\section{Efficiency of a ballistic electrostatic generator: high-speed optical characterization of micro droplets in flight.}

The strong demand for renewable energy promotes research on novel methods and technologies for energy conversion. Microfluidic systems for energy conversion by streaming current are less known, and the relatively low efficiencies previously obtained seemed to limit the further applications of such systems. Here we report a microdroplet-based electrostatic generator operating by an acceleration-deceleration cycle ('ballistic' energy conversion), and show that this principle enables both high efficiency and a compact simple design. Water is accelerated by pumping it through

${ }^{*}$ This chapter is based on:

- Yanbo Xie, Diederik Bos, Lennart de Vreede, Hans de Boer, Mark-Jan van der Meulen, Michel Versluis, Ad Sprenkels, Albert van den Berg and Jan Eijkel, High-efficiency ballistic electrostatic generator using microdroplets, Nat. Commun. 5 (2014).

- Diederik Bos, Yanbo Xie, Mark-Jan van der Meulen, Michel Versluis, Albert van den Berg and Jan Eijkel, Ballistic energy conversion: physical modeling and optical characterization, (submitted, 2014).

${ }^{\dagger}$ The optical characterization, leading to the understanding and magnitude of the losses in the system are part of this thesis. The electrical characterization and modeling were performed by Y. Xie and D. Bos. 
a micronozzle to form a microjet breaking up into fast-moving charged droplets. The droplet kinetic energy is converted in electrical energy when the charged droplets decelerate in the electrical field that forms between the membrane and the target. We experimentally demonstrate conversion efficiencies of up to $48 \%$ with a $30 \mu \mathrm{m}$ diameter nozzle. We also show power density of $160 \mathrm{~kW} / \mathrm{m}^{2}$ and both high- $(20 \mathrm{kV})$ and low- $(500 \mathrm{~V})$ voltage operation.

We characterize the physical behavior of the microjet, the charged microdroplets formed and their subsequent aerodynamic flight towards the charge collection target, with a high-speed setup. From this we calculate the efficiency of the conversion stages. An empirical model is created for the different stages, based on the optical characterization and numerical simulations, from which we predict the optimal working regime. The model predicts a total energy conversion efficiency up to a $40 \%$ for a $10 \mu \mathrm{m}$ nozzle and $70 \%$ efficiency for a $30 \mu \mathrm{m}$ nozzle. The energy conversion efficiency is discussed as a function of the applied pressure, nozzle to target distance, nozzle size and the optimal electrical field during operation, which can assist in predicting and optimizing the proposed ballistic energy conversion system as a method for low-cost and robust renewable energy conversion.

\subsection{Introduction}

Electrostatic generators historically precede electromagnetic generators, and are still used for high-voltage applications [86]. Their working principle is invariably based on the transport of a solid or liquid medium with adsorbed charge to a location of higher electrical potential by continuous application of a mechanical force, typically with low efficiency [87]. With the development of microfluidic and nanofluidic techniques, it has become possible to manipulate the physicochemical processes by a very high surface to bulk ratio. Energy conversion from a streaming current is one of the processes that benefits from this high ratio, and when properly optimized, nanofluidic techniques with overlapping electrical double layers (EDL) $[88,89]$ are now capable of reaching 3 to $5 \%$ energy conversion efficiency, and nearly $11 \%$ in a microjet system $[90,91]$.

In this chapter a high-speed optical characterization study has been performed on a microfluidic electrostatic generator to help quantify its efficiency. The generator works on a novel principle, termed ballistic energy conversion [92]. The system can be classified as an electrostatic generator (like the van de Graaff generator), but unlike all other electrostatic generators it is based on an acceleration/deceleration cycle, effected by jetting high-velocity charged droplets to a target at high potential. We discuss the energy conversion in section 4.2.

We report on electric experiments using $10 \mu \mathrm{m}$ diameter nozzles, in section 4.3 where a maximal efficiency of $36 \%$ was obtained. We demonstrate operation both 
in high voltage, low-current mode and low voltage, high-current mode. We then show measurements on a, with our physical model, optimized system with a $30 \mu \mathrm{m}$ diameter nozzle and we obtained a maximal efficiency of $48 \%$.

We look into the energy losses for the different stages of the energy conversion in section 4.4. Using a high-speed imaging set-up we characterize the physical behavior of the microjet, the charged microdroplets formed and their subsequent aerodynamic flight towards the charge collection target. Using the measurements and numeric modeling a physical model of ballistic energy conversion is built. The model predicts up to a $40 \%$ total energy conversion efficiency when using a $10 \mu \mathrm{m}$ nozzle, increasing to $70 \%$ efficiency for a $30 \mu \mathrm{m}$ nozzle, as a result of the lower surface to bulk ratio and resulting lower energy losses in the larger nozzles.

\subsection{Energy conversion}

The energy conversation principle of the generator is shown in Fig. 4.1. A highspeed $(10 \mathrm{~m} / \mathrm{s})$ microjet is formed by forcing pressurized water through a micro nozzle. The jet breaks up into a droplet train by the Rayleigh-Plateau instability. The droplets carry a net positive ionic charge. This charge originates from advection of the positive mobile ions in the electrical double layer (EDL) of the membrane (the streaming current phenomenon), but can be strongly increased by induction by applying a negative potential to the metal 'guard ring' located close to the nozzle exit. The droplets travel through air towards a metal target that acquires a high electrical potential by charge accumulation owing to droplet impact. Along the droplet trajectory, the high electrical field between target and jet decelerates the droplets at $\sim 10^{4} \mathrm{~g}$. As the droplets move to a location of higher electrical potential, their kinetic energy is directly converted into electrical energy ('ballistic energy conversion') due to the force balance $m a=-q E$ with $m$ and $q$ the droplet mass and charge, $a$ the acceleration and $E$ the electrical field and for the moment neglecting air drag. Current is drawn from the high potential target to perform useful work. The function of the guard ring is to control the local electrical field: when grounded, it prevents induction of negative charge in the jet by the positively charged target, and when it is held at a negative potential, it induces additional positive ionic charge in the jet. It should be emphasized that the induction process by the guard ring does not consume energy, as no current flows through the guard ring under normal operation. The system operates by physics that fundamentally differs from the physics governing both classical fluidic electrokinetic energy conversion systems and electrostatic generators [93-97]. It was inspired by prior work on microjet energy conversion [90, 91, 98]. However, the electrostatic induction of charge allows operation independently of the EDL on the membrane surface, enabling an increased droplet charge density and thus a lower target voltage. This offers great advantages for future applications. 


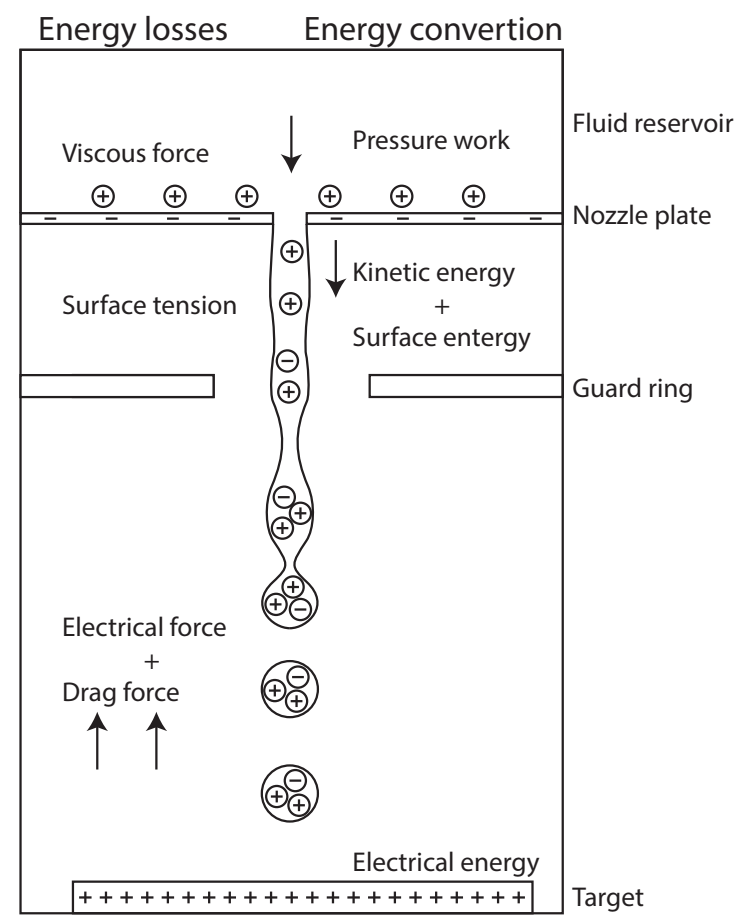

Figure 4.1: Conceptual illustration of the conversion principle. Water is accelerated to form a microjet that breaks up into charged droplets. On their air trajectory towards the target, the droplets are decelerated by the electrical force, converting kinetic energy into electrical energy.

\subsection{Electrical characterization}

\subsubsection{Device and set-up}

By applying air pressure of $\sim 100 \mathrm{kPa}$, an aqueous solution is forced through a 10 or $30 \mu \mathrm{m}$ diameter circular nozzle etched in a $500 \mathrm{~nm}$-thick silicon nitride membrane, see Fig. 4.2. A microjet is formed that breaks up in a droplet train. The droplets travel through the air and arrive on a metal target placed at a distance ranging from $7.5-25 \mathrm{~mm}$ from the nozzle opening. The target is connected to electrical ground via a high-load resistance $(1-10 \mathrm{~T} \Omega)$. A metal guard ring with an opening of $2 \mathrm{~mm}$ diameter is placed at a distance $1.5 \mathrm{~mm}(10 \mu \mathrm{m}$ nozzle $)$ or $5.0 \mathrm{~mm}$ ( $30 \mu \mathrm{m}$ nozzle) from the nozzle exit and was kept at ground potential, or at a negative potential for inductive droplet charging.

A platinum electrode sputtered on the backside of the chip ( $30 \mu \mathrm{m}$ nozzle experiments) or an inserted platinum wire ( $10 \mu \mathrm{m}$ nozzle experiments) was connected to a picoammeter (Keithley 6485). The following currents were measured: $I_{1}$ between 


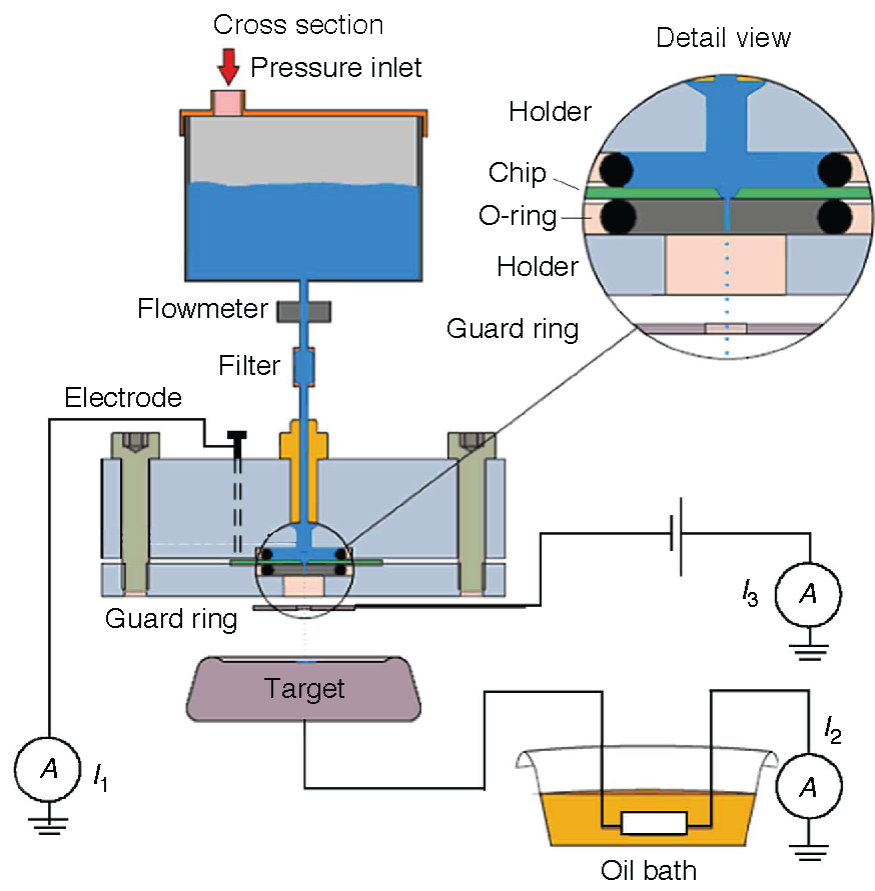

Figure 4.2: Experimental set-up for the electrical characterization. The aqueous solution is forced by applied air pressure through a 10 or $30 \mathrm{~mm}$ diameter nozzle in a thin membrane. The current can be measured between ground and the reservoir electrode $\left(I_{1}\right)$, between target and ground $\left(I_{2}\right)$ and between guard ring and ground $\left(I_{3}\right)$.

ground and the platinum reservoir electrode, $I_{2}$ between the target and ground and $I_{3}$ between the metal guard ring and ground. The electrical target potential is generated by a high voltage source (FUG - HCE 7 35000), instead of using a series resistance, since it can provide the electrical voltage without delays caused by the target capacitance.

\subsubsection{Results}

For a typical experiment with a $10 \mu \mathrm{m}$ diameter nozzle and demineralized water, Fig. 4.3(a) shows the upstream current $I_{1}$, from grounded reservoir electrode to jet, and the downstream current $I_{2}$, from target to ground. At $t=220 \mathrm{~s}$ the system is pressured. When jetting starts, charge flows out of the reservoir as indicated by $I_{1}$. The exponential increase of $I_{2}$ is determined by the electrical RC time (product of target capacitance $\mathrm{C}$ and load resistance $\mathrm{R}$ ). The steady-state difference between both currents is caused by the reflection of charged droplets from the target at high potential, see Fig. 4.5, landing on the grounded guard ring and generating current $I_{3}$. 


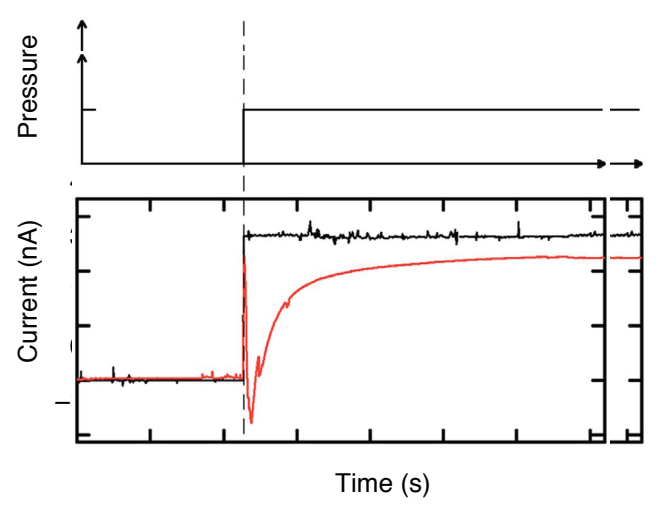

(a)

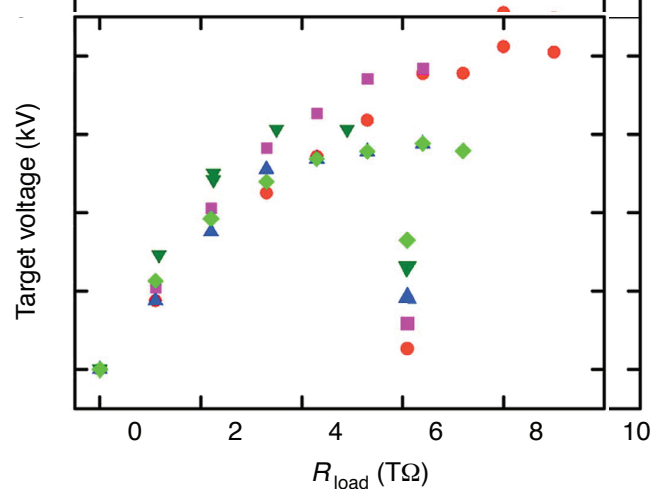

(c)

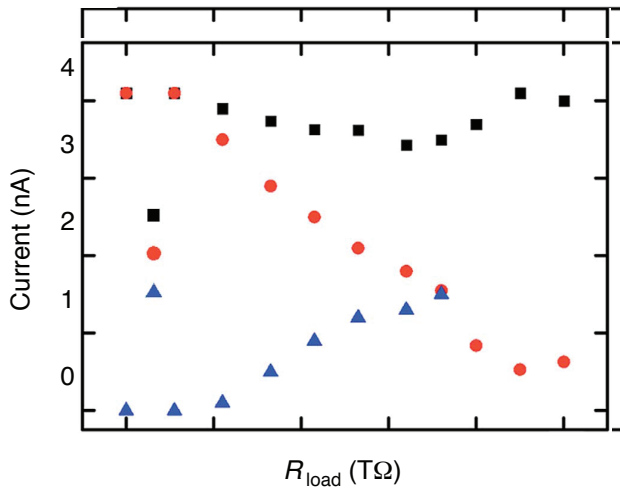

(b)

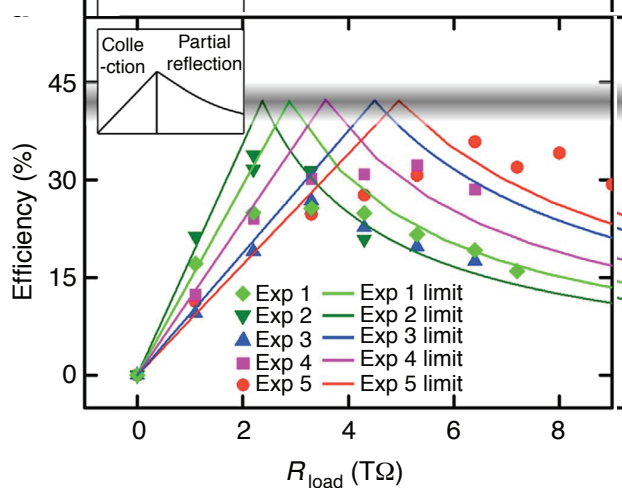

(d)

Figure 4.3: Experimental results using $10 \mu \mathrm{m}$ diameter nozzles. (a) Upstream $\left(I_{1}\right)$ and downstream $\left(I_{2}\right)$ current on application of pressure, using a grounded guard ring. (b) Upstream, downstream and guard ring current $\left(I_{3}\right)$ with increasing value of the load resistance. (c) Generated target voltage as function of load resistance for different upstream currents. (d) Conversion efficiency as a function of load resistance for different values of the upstream current. Experimental values (symbols) and theoretical predictions (lines) are shown, as well as the theoretical maximum efficiency of $37-43 \%$ (depending on flow rate). 


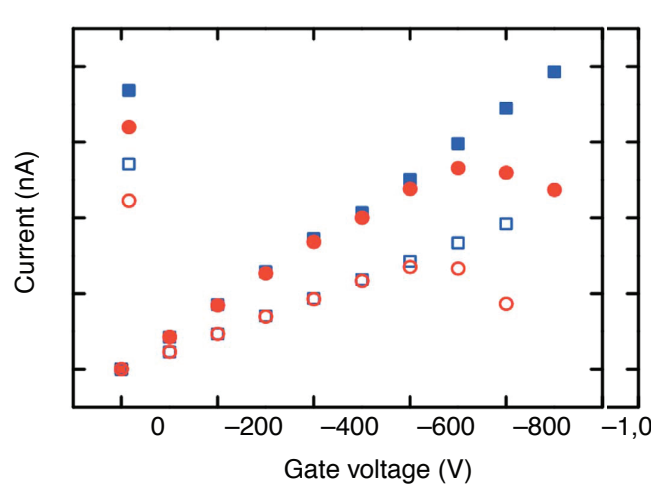

(a)

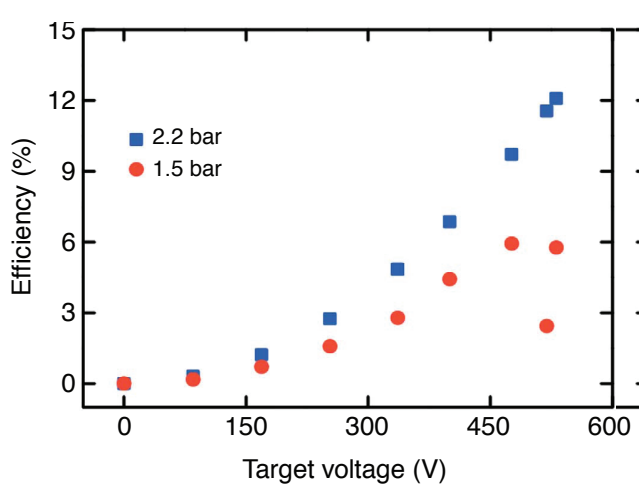

(b)

Figure 4.4: High current and low-voltage operation. Current as function of gate voltage (a) and as function of generated target voltage (b).

Figure 4.3(b) displays the currents $I_{1}, I_{2}$ and $I_{3}$ for a typical measurement in which the load resistance is increased in steps of $1 \mathrm{~T} \Omega$. $I_{1}$ remains approximately constant, while $I_{2}$ decreases with the load resistance and $I_{3}$ increases from initially zero. Figure 4.3(c) plots the target voltage, calculated as the product of the harvested current $I_{2}$ and load resistance as $V_{\text {target }}=I_{2} R_{\text {load }}$ against load resistance for a number of experiments. The target voltage increases with load resistance up to anything between 10 and $16 \mathrm{kV}$, where it is found to saturate. The conversion efficiency from mechanical to electrical energy, $E f f=I_{2} V_{\text {target }} /(p Q)$, with $p$ the applied reservoir pressure and $Q$ the volumetric flow rate. The conversion efficiency was found to increase with load resistance Fig. 4.3(d), for $10 \mu \mathrm{m}$ nozzles reaching a typical efficiency around $33 \%$ with a maximum value of $36 \%$.

On the basis of the acceleration/deceleration mechanism, we expect two regimes of operation, which can be observed in Fig. 4.3(c,d). At low values of the load resistance, the target potential $V_{\text {target }}=I_{2} R_{\text {load }}=I_{1} R_{\text {load }}$ is low and the kinetic energy of the droplets is only partially converted into electrical energy. In this 'collection regime', the target potential linearly increases with the load resistance, as does the efficiency $E f f=\left(I_{1}\right)^{2} R_{\text {load }} /(p Q)$. Increasing the load resistance, the target potential at constant $I_{2}=I_{1}$ will reach a value where droplets will only just be able to reach the target with the kinetic energy available. The optimum potential that can be reached is $V_{\text {opt }}=\left(E f f_{\text {max }}\right) p Q / I_{1}$, and is generated at load resistance $R_{o p t}=V_{o p t} / I_{1}$. For our experimenten we find for the $10 \mu \mathrm{m}$ nozzle $E f f_{\max }=0.40$. In various experiments $V_{\text {opt }}$ varied between 11.5 and $17 \mathrm{kV}$, Fig. 4.3(c). When the resistance is further increased, an increasing fraction of the droplets is observed to be reflected from the target as can be seen in Fig. 4.5. In this 'partial reflection regime' $I_{2}$ becomes progressively smaller than $I_{1}$ with increasing $R_{\text {load }}$ (Fig. 4.3(b). Assuming that all droplets have 
identical charge to mass ratio, $I_{2}$ in this regime will be inversely proportional to $R_{\text {load }}$ since $V_{\text {target }}=V_{\text {opt }}=I_{2} R_{\text {load }}$, and $E f f=E f f_{\text {max }} R_{\text {opt }} / R_{\text {load }}$.

The experiments in Fig. 4.4(a/b) show operation at high voltage and low-current. Although useful for some applications, many applications will benefit from lowvoltage and high-operating current. From the equality $\frac{1}{2} m v^{2}=q V_{\text {target }}$, it follows that this can be achieved by increasing the droplet charge density $q / \mathrm{m}$. Using inductive charging and a more conductive $0.1 \mathrm{M} \mathrm{KCl}$ solution, we could operate a single nozzle at $500 \mathrm{~V}$ target voltage and $50 \mathrm{nA}^{\ddagger}$ current with $12 \%$ efficiency, Fig. 4.4(a,b).

In the analysis of the energy losses in the system, discussed in the next section, the efficiency was found to increase for larger nozzle diameters. To still decelerate the now heavier droplets at practical target potentials $(<20 \mathrm{kV})$, a larger droplet charge needs to be induced. Experiments were performed with a $30 \mu \mathrm{m}$ nozzle in combination with inductive charging by a guard ring voltage using a more conductive $10 \mathrm{mM} \mathrm{KCl}$ solution. Figure 4.6(a,b) shows the results of two experiments, plotting currents and efficiency against induction voltage and increased conversion efficiency of $48 \%$ was measured.

\footnotetext{
†The droplet charge density obtained in these microdroplets was about half the Rayleigh limit (the Rayleigh limit describes the droplet charge at which Coulomb repulsion overcomes surface tension and droplets become unstable. It equals $\pi \sqrt{8 \sigma \varepsilon_{0} d^{3}}$ Coulomb, where $\sigma$ is the surface tension $(72 \mathrm{mN} / \mathrm{m}$ for water), $\varepsilon_{0}$ the permittivity of free space and $d$ the droplet diameter).
} 


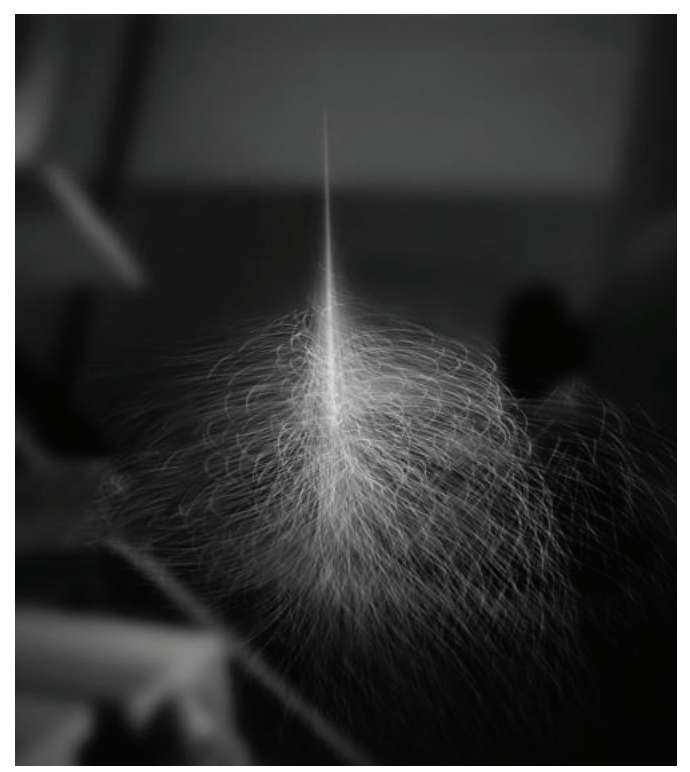

(a)

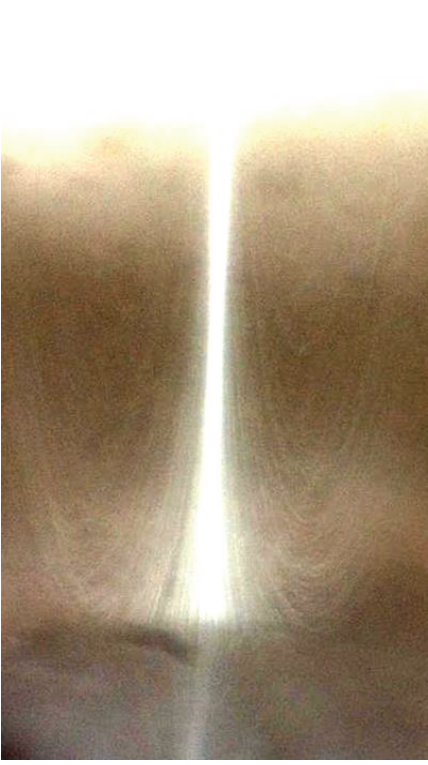

(b)

Figure 4.5: (a) Microdroplets shooting in the open air, the drops reach their terminal velocity and deflect from the straight path. (b) Microroplets reflected at the charged target; a portion of the droplets are accelerated away from the target.

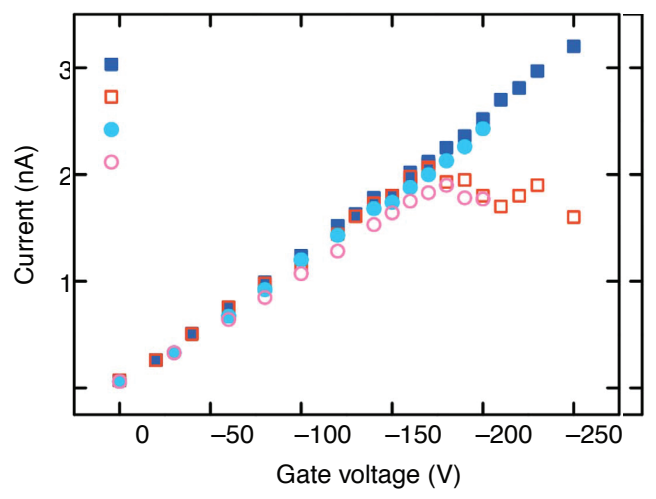

(a)

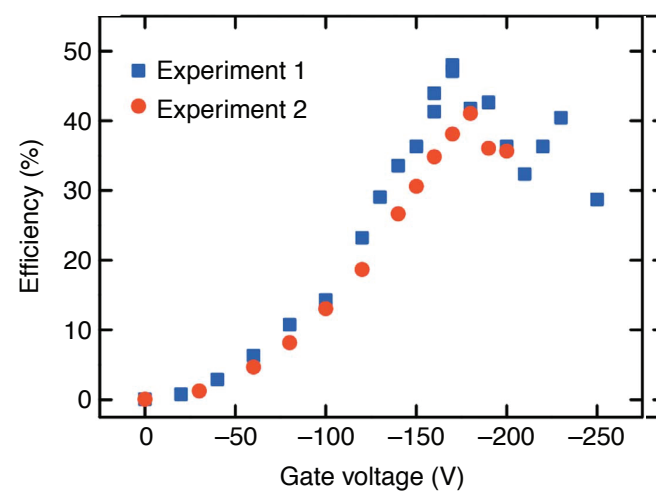

(b)

Figure 4.6: Two experiments using a $30 \mu \mathrm{m}$ diameter nozzle. (a). Currents $I_{1}$ and $I_{2}$ as a function of gate voltage. (b) Efficiency as a function of gate voltage with a $1.01 \mathrm{~T} \Omega$ load resistance. Applied pressure 1.38 bar, average flow rate $6.55 \mu \mathrm{Ls}$, solution $10 \mathrm{mM}$ $\mathrm{KCl}$, target distance $2.5 \mathrm{~cm}$ (experiment 1 ) and $2.0 \mathrm{~cm}$ (experiment 2). 


\subsection{Energy conversion losses}

The efficiency is limited by losses in the system. The aim of this section is to identify and to quantify fluidic and aerodynamic losses. An optical characterization of the system improves the understanding of the ballistic energy conversion process and losses. Using this information, in conjunction with numerical simulations, a model was built to improve the design of the system by predicting its optimal performance and optimal operational conditions. Here the droplet speed as a function of the applied pressure, its trajectories and flight distances are analyzed to find the energy losses in the fluidic and ballistic stages. We discuss the energy conversion efficiency as a function of the nozzle size, its optimal flight distance and corresponding electrical field, indicating ways to even further increase the conversion efficiency.

The conversion efficiency is defined in two stages. In stage 1 the work done by pressure is converted to droplet kinetic energy with an efficiency $E f f_{k i n}$. The applied pressure accelerates the fluid through a micronozzle to form a liquid microjet. Significant energy losses in this stage are the viscous friction when the liquid flows though the micronozzle [99], and the energy needed to create the air/water interface of the cylindrical liquid jet breaking up into droplets [100]. The experimental verification of these losses on this stage can be found in sections 4.4.2 \& 4.4.3. In stage 2, droplet kinetic energy is converted in electrical energy with an efficiency $E f f_{e l}$. The charged droplets move with an initially high velocity against the electrical field and in addition undergo air drag, the most significant loss in this stage. The measurements and model on stage 2 can be found in section 4.4.4.

Finally, the overall efficiency of ballistic energy conversion can be calculated as:

$$
E f f=E f f_{k i n} \cdot E f f_{e l} .
$$

If all other sources of energy loss are insignificant, this theoretical outcome is equal to the measured electrical energy conversion efficiency $E f f=(U I) /(p Q)$.

\subsubsection{Optical experimental set-up}

To quantify the energy loss in each stage, the microjet size and droplet velocity must be known at several heights from the nozzle. The optical characterization set-up (Fig. 4.7(a) was used to capture images of droplets in flight at variable distances from the micronozzle. During the experiments also pressure, flow rate and the electrical current flowing from the reservoir were measured. The nozzle diameters of $10 \mu \mathrm{m}$ demand a resolution of approximately $1 \mu \mathrm{m} / \mathrm{pixel}$, to resolve the main and even smaller satellite drops $[10,101]$. Taking into account the high droplet velocities that are obtained with continuous-pressure drop-formation devices, of about $20 \mathrm{~m} / \mathrm{s}$, this requires a maximal illumination time of not more than $50 \mathrm{~ns}$. 
Since the breakup frequency is not set externally, as with for example DOD inkjet printing, the velocity can not be determined directly from the displacement of two subsequent droplets in a single frame. Also the drop-to-drop velocity variations will result in a significant reduction of the accuracy. Therefore the recorded frames were double-illuminated to capture the same drops at two instances in time. From the displacement over this exact time the velocity of the drops was determined. A dual cavity laser source (Litron Nano-PIV Q-switched doubled Nd:YAG laser, 7 ns laser pulse) coupled into a fluorescent diffuser (Lavision diffuser) provides two non-coherent light pulses with 1-2 $\mu$ s delay during a single exposure of the camera (Lumenera $\mathrm{Lm} 135,4.65 \mu \mathrm{m}$ square pixel size). For the experiments the Olympus SLMPLN $5 \times$ and $10 \times$ objectives were used. The laser trigger time delay and camera triggering were controlled by a pulse/delay generator (Berkeley Nucleonics BNC 575; 250 ps accuracy). A sample image is shown in Fig. 4.7(b).

\subsubsection{Jet formation: viscous friction}

The viscous losses during jet formation and the opposing Laplace pressure of the formed jet, reduce the effective pressure to drive the flow in the jet $[102,103]$. The kinetic energy of the jet can not be determined from the optical characterization alone, because it is not possible to determine the jet velocity with the double illuminated frames. The flow rate of the jet was determined using a flowmeter at the system inlet. The measured flow rate for different pressures are plotted in Fig. 4.8. The optical characterization set-up was used to determined the jet diameter. A recording of a jet emerging from the $30 \mu \mathrm{m}$ nozzle is shown in Fig. 4.8a. For the $10 \mu \mathrm{m}$ nozzles $a / R=0.9$ was found and for the $30 \mu \mathrm{m}$ nozzles $a / R=0.8$ was found, under our applied experimental conditions.

The viscous energy losses of a liquid flowing through an orifice can be calculated analytically for low Reynolds numbers by the creeping flow model originally developed by Sampson [102]. Alternatively, the energy loss factor can be taken from empirical data on the head loss or pressure loss in a small opening into a circular pipe [104]. The Reynolds number in the present system, however, is close to 100 which does not satisfy the creeping flow assumption. In addition, the water passing through the micron-sized nozzle forms a free liquid jet in air, which is fundamentally different from the liquid flow in a pipe. Hence, we used numerical simulations of the water flow to estimate the viscous energy loss in our system. The input mechanical power equals $P=p Q$, with $p$ and $Q$ the input pressure and volume flow rate respectively, where for incompressible flow $Q$ stationary at every position. The pressure that is effectively used to drive the flow and to form a jet, $p_{e f f, \text { jet }}$, equals the input pressure minus the pressure losses due to viscous friction, $\left(p_{v i s}\right)$, and due to surface tension, $\left(p_{s}\right)$ :

$$
p_{e f f, j e t}=p-p_{v i s}-p_{s}
$$




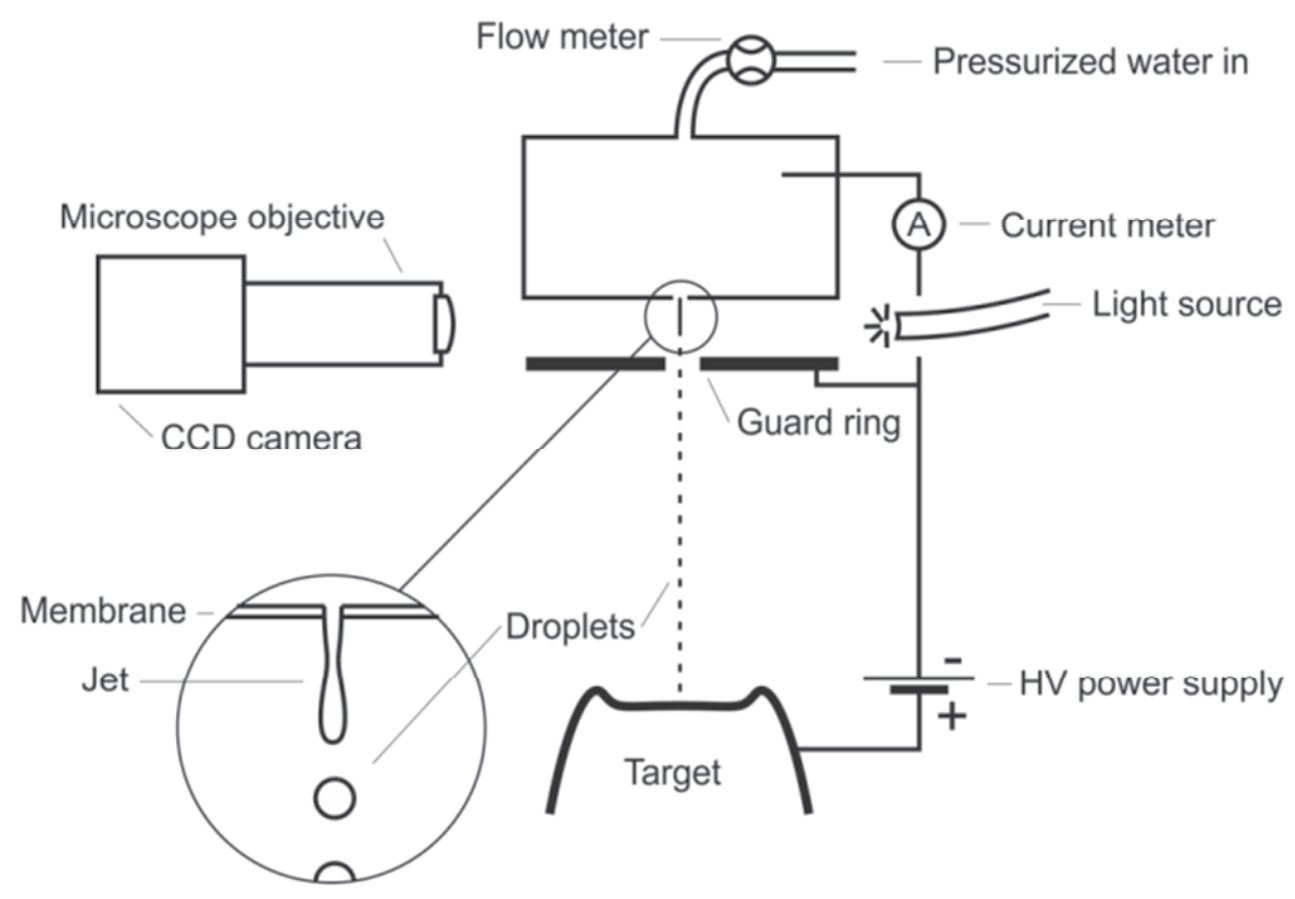

(a)

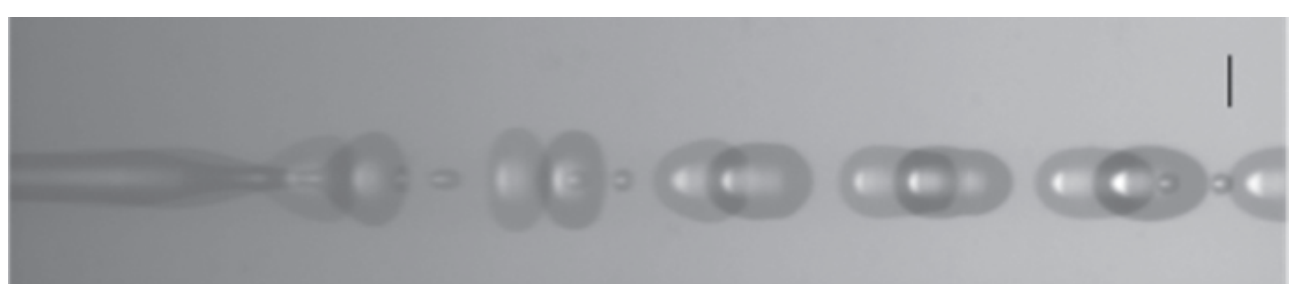

(b)

Figure 4.7: (a) Setup for optical velocity measurements. Flow meter and water inlet are electrically isolated from the set-up. (b) Sample picture by double illumination of droplets from the droplet train from a $30 \mu \mathrm{m}$ nozzle. The scale bar indicates $25 \mu \mathrm{m}$. 
$p_{s}$ represents the Laplace pressure of the cylindrical liquid jet expressed as $\gamma / a$, where $\gamma$ is the surface tension and $a$ is the radius of the jet. The viscous pressure loss $p_{v i s}$ scales with the shear rate, and thus, for laminar flow, it is also inversely proportional to the jet radius a. This loss scales with the input pressure, and can be written as $p_{v i s}=$ $p(K / a)$, where $K$ is defined as a constant loss factor for the viscous loss, independent of $p$ and $a$. The effective pressure can now be expressed as:

$$
p_{e f f, j e t}=p(1-K / a)-\gamma / a
$$

The observed jet radius $a$ is typically smaller than the nozzle radius due to the contraction of the jet. The contraction ratio of the liquid jet is influenced by many factors, such as the nozzle size and shape, its surface roughness and the velocity profile [105]. The loss factor $K$ was estimated from the flow profile by a numerical simulation in COMSOL Multiphysics using a two-phase flow model. To exclude surface tension effects, the surface tension was reduced by a factor 20 , so that only viscous friction played a significant role. Simulations then yielded a value for $K$ of $1.30 \mu \mathrm{m}$, determined as an average over simulations with several jet diameters ranging from $8 \mu \mathrm{m}$ to $30 \mu \mathrm{m}$. The effective pressure (the pressure that contributes to the kinetic energy of liquid flow) can be used to theoretically predict the flow rate $Q$ using the Bernoulli equation. The jet velocity $v_{j}$ and the flow rate $Q$ as function of $p_{e f f, j e t}$ are

$$
\begin{gathered}
v_{j}^{2}=\frac{2 p_{e f f, j e t}}{\rho_{w}} \\
Q=\pi a^{2} v_{j}
\end{gathered}
$$

We found that the theoretically predicted flow rate $Q$ is in good agreement with our measured $Q$ as a function of the pressure for $10 \mu \mathrm{m}$ and $30 \mu \mathrm{m}$ nozzles, as shown in Figs. 4.8.

\subsubsection{Jet breakup: surface energy and the momentum balance}

The size distribution of the drops was evaluated shortly after the jet has broken up and about $1.5 \mathrm{~cm}$ below the nozzle, see Fig. 4.9. Directly after the drop formation a bimodal distribution is found for the $30 \mu \mathrm{m}$ nozzle, due to the main drops and satellite drops which emerged from the breakup of the filaments between the drop. For the $10 \mu \mathrm{m}$ nozzles, the satellites were not analyzed, because their size was below the optical resolution provided by the imaging system. Downstream of the jet breakup point he nozzle, the drops and satellite drops have coalesced and the the average drop sizes are larger.

The energy conversion efficiency of the applied work to the droplets can be determined using the kinetic energy of the drops directly after the breakup of the jet. This velocity is determined using the double illuminated frames and is evaluated at the 


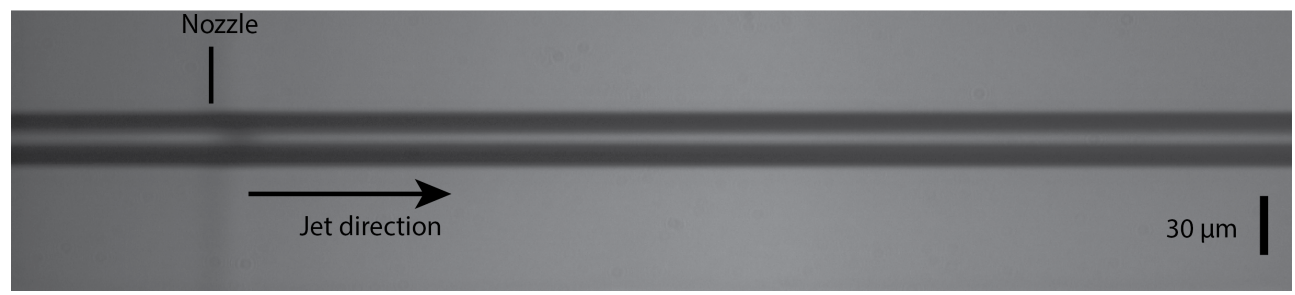

(a)

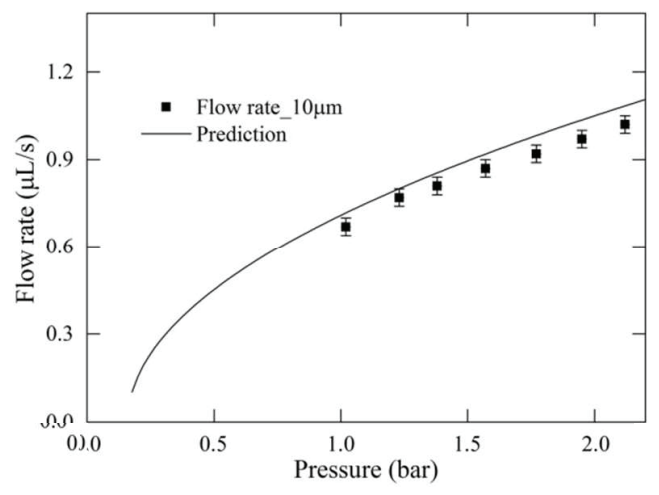

(b)

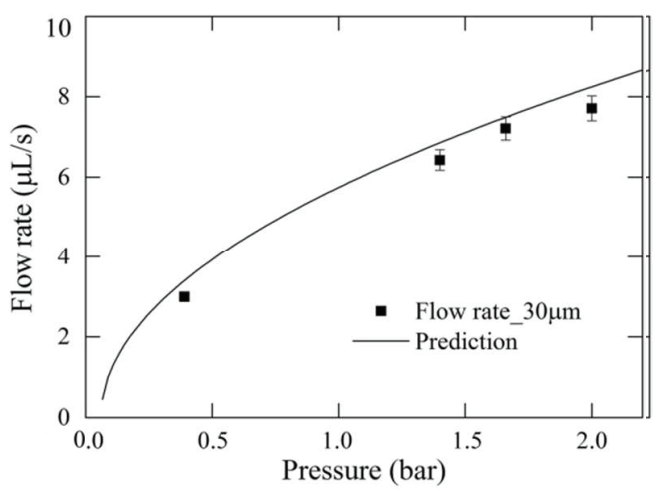

(c)

Figure 4.8: (a) Jet from $30 \mu \mathrm{m}$ nozzle. The jet diameter was found to be $24 \mu \mathrm{m}$. (b) Observed and predicted flow rate $Q$ for several pressures using a $10 \mu \mathrm{m}$ diameter nozzle (a) and $30 \mu \mathrm{m}$ diameter nozzle (c). The theory is based on observed jet radii a of $4.5 \mu \mathrm{m}$ and $12 \mu \mathrm{m}$ respectively

first drops that have formed, see Fig. 4.10a. The measured drop velocity as a function of the applied pressure, is plotted in Fig. 4.10(b/c).

Interfacial effects impact both the flow rate from the nozzle (due to the Laplace pressure) and the breakup of the droplets. Assuming the jet and droplets are axisymmetric, the interfacial forces are balanced in the radial orientation and the Laplace pressure reduces the net pressure exerted on the fluid, as in Eq. 4.3. At the position of jet breakup, the Laplace pressure yields a positive contribution to the kinetic energy of the droplets, but the interfacial forces are not balanced in the axial orientation, which yields a negative contribution to the droplet kinetic energy [99]. If we define an artificial surface at the position where the jet just pinches off, this surface is 'pulled' by the jet surface force but 'pushed' by the unbalanced Laplace pressure. This phenomenon was described and measured by Schneider $e$ t al. and the velocity was derived from a force balance [99]:

$$
\frac{d\left(M_{j}\right)}{d t}=\frac{d\left(M_{d r}\right)}{d t}+\pi a^{2} p-2 \pi a \gamma
$$




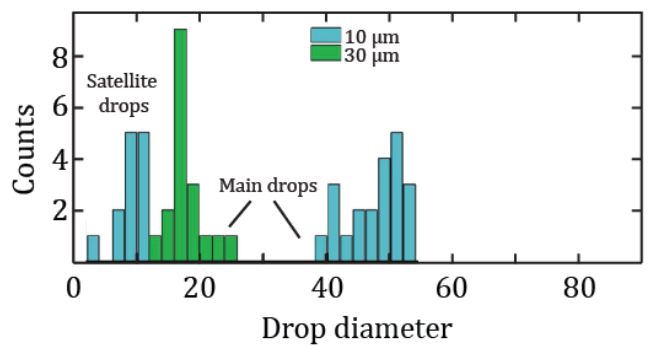

(a)

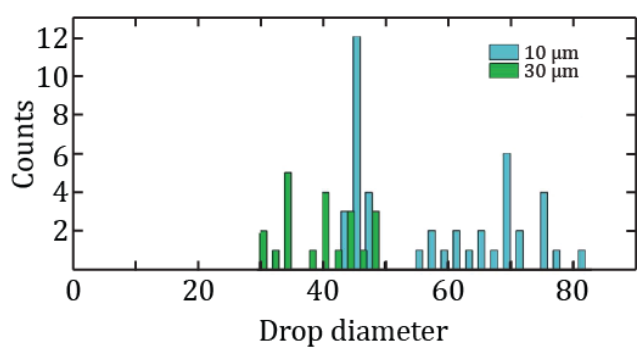

(b)

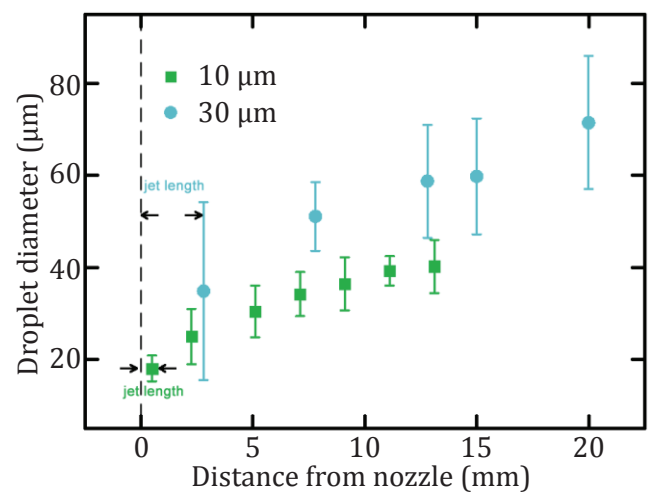

(c)

Figure 4.9: Size variation of droplets. (a) The size distribution obtained at the point where the jet breaks up into droplets. The data give a clear indication of a bimodal distribution with small satellite droplets and larger main drops. (b) The size distribution of droplets at $1.3 \mathrm{~cm}(10 \mu \mathrm{m}$ nozzle $)$ and $1.5 \mathrm{~cm}(30 \mu \mathrm{m}$ nozzle) distance from the nozzle. Both nozzles produce main and satellite droplets, however the satellite droplets from the $10 \mu \mathrm{m}$ nozzle are too small to be analyzed. Main and satellite droplets coalesce during the flight, therefore no satellite droplets were observed at $1.5 \mathrm{~cm}$ below the nozzle, and the average droplet sizes have increased. (c) The average drop diameter at different positions from the nozzle, with a $10 \mu \mathrm{m}$ diameter nozzle (green) and a $30 \mu \mathrm{m}$ diameter nozzle (blue). 
Where $M_{j}, M_{d r}$ are the momentum of the liquid in the jet and in the droplets, respectively. Working out the equation gives the initial velocity of the drops after breakup of the jet [99]:

$$
v_{0}=v_{j}\left(1-\frac{\gamma}{\rho_{w} a v_{j}^{2}}\right)
$$

Substituting Equation 4.3 and 4.4 in Equation 4.7 we can calculate the droplet velocity as a function of the applied pressure. The energy conversion efficiency in the droplet generation stage can then be calculated as:

$$
E f f_{\text {kin }}=\frac{v_{0}^{2}}{v_{\text {lossless }}^{2}}=\left(\left(1-\frac{K}{a}\right)-\frac{\gamma}{p a}\right)\left(1-\frac{\gamma}{2(p(a-K)-\gamma)}\right)^{2} * C
$$

The first term of Eq. 4.8 represents the losses during jet formation, and the second term indicates the losses due to the jet breakup. Fig. 4.10 shows that this model, with a loss factor $C=0.94$, nicely follows the measured droplet velocities as a function of the applied pressure. The efficiency $E f f_{k i n}$ increases with the applied pressure, and it is also apparent from Eq. 4.8 that the use of a larger nozzle leads to a decrease of the energy loss during droplet generation. Increasing the applied pressure beyond $120 \mathrm{kPa}$ will not significantly contribute to a decrease of the energy loss in the droplet generation process, see Fig. 4.10(c).

\subsubsection{Air drag}

After the jet breaks up into droplets, the charged droplets are slowed down by the electrical field while moving towards the high-potential target, thus converting kinetic energy into electrical energy. In addition, air drag also y consumes kinetic energy. The velocity of the droplets have been measured for increasing distances from the nozzle. Further away from the nozzle, the average drop size increases due to coalesce and merging with satellite droplets, see Fig. 4.11(a). The results are plotted in Fig. 4.11(b/c). The velocity is observed to decrease, which is caused by the opposing electrical field and in addition the air drag on the drops.

The motion of droplets during their trajectory from nozzle to target is described by Newton's second law:

$$
\begin{gathered}
F=m \frac{d v}{d t}=F_{e l}+F_{d r} \\
v \frac{d v}{d x}=\frac{F_{e l}}{m}+\frac{F_{d r}(x)}{m}
\end{gathered}
$$

The motion of the droplets can be calculated from the electric force $F_{e l}$ and the drag force $F_{d r}$ as a function of the trajectory position $x$. The first term of deceleration 


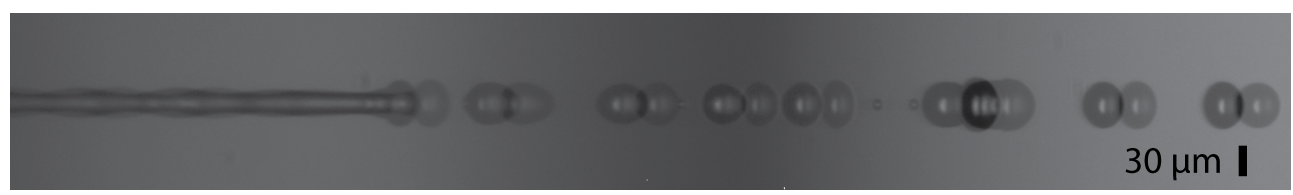

(a)

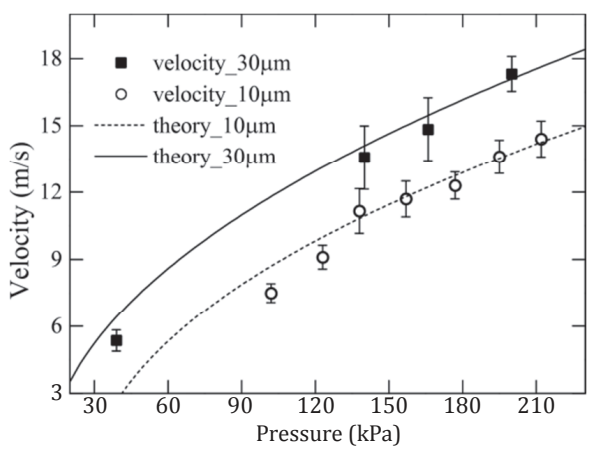

(b)

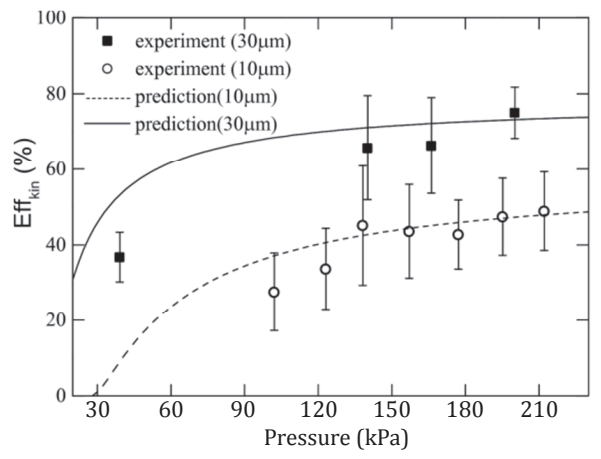

(c)

Figure 4.10: (a) Droplet formation from $30 \mu \mathrm{m}$ nozzle. The frame is double illuminated with a time delay of $2 \mu \mathrm{s}$. (b) Velocity of the initial droplet as function of applied pressure. The initial velocity of droplets were measured at the position where jet just breaks up from $10 \mu \mathrm{m}$ and $30 \mu \mathrm{m}$ nozzles, respectively. (c) By measurement of initial droplets velocity, the efficiency of pressure to kinetic energy conversion $\left(E f f_{\text {kin }}\right)$ can be calculated. The data points indicate the measurement results and the lines are from.

due to the electrical field $F_{e l} / m$ can be calculated from the droplet charge density and the electrical field strength, assuming the electric field is constant:

$$
C_{e l}=\frac{F_{e l}}{m}=\frac{U}{l} \frac{\rho_{e l}}{\rho_{\text {water }}}
$$

where $C_{e l}, U, l, \rho_{e l}$, are the electrical deceleration, potential difference, length of the trajectory in the electric field and droplet charge density, respectively.

The air drag force on a single spherical droplet can be expressed as $F_{\text {drag }}=$ $\frac{1}{2} \rho_{\text {air }} v^{2} C_{t r} A$, where $\rho_{\text {air }}, v, C_{t r}$ are the mass density of air, the speed of the droplet relative to air, and the air drag coefficient, respectively [106]. However, here we have a train of droplets, which has three complicating effects: (1) a wake in the surrounding air is developed (2) The observed downstream droplet size increases because of merging of the droplets in flight, see Fig. 4.9, and (3) the distance between the droplets depends both on the velocity of the droplets and the occurrence of merging. We therefore modeled the air drag by fitting to experimental data and numerical simulations. When fitting the experimental data, we found that deceleration scales 
inversely with the root of the distance from the nozzle. We model:

$$
\frac{F_{d r}}{m}=\frac{C_{t r} v^{2}}{\sqrt{x}}
$$

where $C_{t r}$ is an air drag coefficient for drop trains, with unit $x^{-1 / 2}$. The valuer of $C_{t r}$ was obtained from numerical simulations with COMSOL, keeping the train of droplets stationary and moving an air flow around the droplets. The force on the droplets was calculated by integration of the force on the droplet surface, yielding $C_{s t}=5.3 e^{-a / 5.5 e-6}$ from different nozzle sizes, with a maximum $7.7 \%$ deviation when the droplet radius was varied from $8 \mu \mathrm{m}$ to $20 \mu \mathrm{m}$ and when its velocity was varied from $5 \mathrm{~m} / \mathrm{s}$ to $12 \mathrm{~m} / \mathrm{s}$. Measurements of the droplet velocity as a function of the distance from the nozzle are shown in Fig. 4.11. Figure 4.11(a) shows the velocity decrease of droplets from $10 \mu \mathrm{m}$ and $30 \mu \mathrm{m}$ nozzles as a function of the distance from the target, in the absence of an electrical field. To study the deceleration of droplets, where the electrical deceleration factor given by Eq. 4.11 plays a significant role, an additional experiment with an applied electrical field was performed, see Fig. 4.11(b). It can be clearly seen that the droplets decelerate faster when a $7 \mathrm{kV}$ voltage was applied between the target and the guard ring, due to the increased electrical force. The model of Eq. 4.10 which includes both electrical and air drag contributions, shows good correspondence to the experimental data.

\subsubsection{System efficiency}

By combining the equations of energy loss in the two consecutive stages, we can now predict the efficiency of the ballistic energy conversion system as a function of the most important factors such as jet radius $a$ (approximated by $0.9 R$ ), the applied pressure $p$ and the trajectory distance $x$. The velocity of the drops is found by substitution of Eq 4.12 in Eq. 4.10:

$$
v^{2}(x)=\frac{-C_{e l}}{C_{t r}} \sqrt{x}+v_{0}^{2} e^{-4 C t r \sqrt{x}}+\frac{C_{e l}}{4 C_{t r}^{2}}\left(1-e^{-4 C_{s t} \sqrt{x}}\right),
$$

with $v_{0}$, the initial velocity of the drops, after the losses in stage 1 , and is described by Eq. 4.7 under substitution of Eqs. 4.3 and 4.4.

We find the energy conversion efficiency of the system at a distance $L$ from the position of jet breakup:

$$
E f f_{e l}=1-\frac{E_{L}}{E_{0}}=1-\frac{2 C_{t r}}{v_{0}^{2}} \int_{0}^{L} \frac{v(x)^{2}}{\sqrt{x}} d x
$$

where $E_{0}$ and $E_{L}$ are the droplet kinetic energy, just after jet breakup and at distance $L$. 


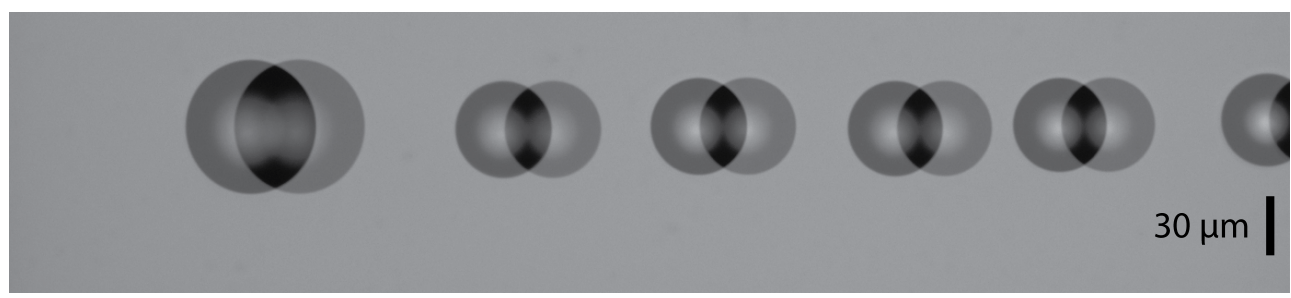

(a)

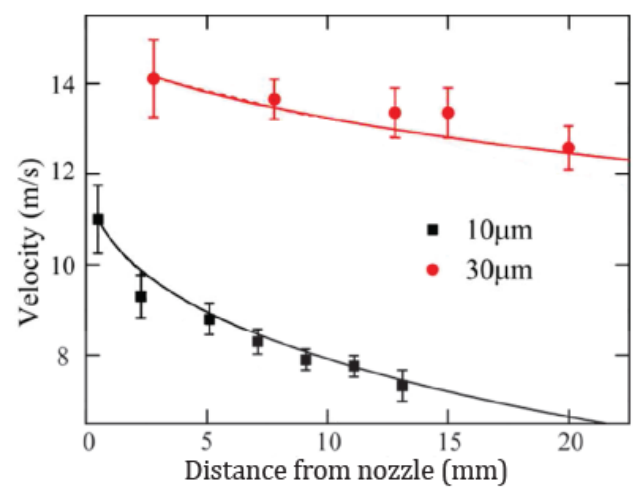

(b)

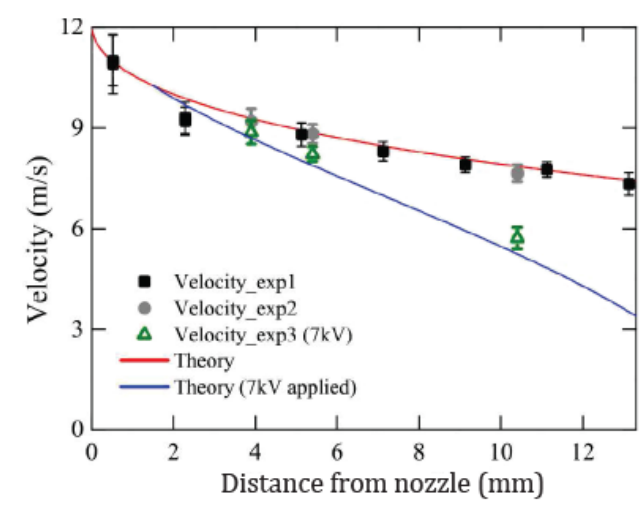

(c)

Figure 4.11: (a) Droplet formation from $30 \mu \mathrm{m}$ nozzle. The frame is double illuminated with a time delay of $2 \mu \mathrm{s}$. (b) The velocity decrease as function of trajectory distance of drops from a $10 \mu \mathrm{m}$ nozzle (black points) and $30 \mu \mathrm{m}$ nozzle (red points) compared with the model. (c) Velocity measurements and predictions as function of distance from the micronozzle. The applied pressure is 1.4 bar and the distance between nozzle and target (for experiment 3) is $13 \mathrm{~mm}$. In exp.3 an electrical field was was applied between $3 \mathrm{~mm}$ (location of the guard ring) and $13 \mathrm{~mm}$ (the location of the target).

\subsubsection{Optimal working parameters}

The maximum output power of the system is obtained when the droplets convert all their kinetic energy to electrical energy, with minimal losses before hitting the target. Hence, we can calculate the maximum electrical field by defining $v(L)=0$, where $L$ is the maximum distance the droplets can reach under exertion of the electrical field force $F_{e l}$ and air drag force $F_{d r}$. This yields the required maximum electrical field from the electrical deceleration term as function of guard ring-target distance $x$ :

$$
C_{e l, \max }=\frac{x-x_{0}}{L} U \rho_{e}=\frac{4 v_{0}^{2} C_{t r}^{2} e^{-4 C_{t r} \sqrt{x}}}{-1+4 C_{t r} \sqrt{x}+e^{-4 C_{t r} \sqrt{x}}}
$$

Finally, the system efficiency can be calculated with Eqs. 4.1, which terms are given by Eqs. 4.8 and 4.14. The optimal efficiency and working electrical field derived 
from Eq. 4.15 are shown in Fig. 4.12.

In practical circumstances however, an upper limit is posed to the field strength due to the electrical breakdown of air $(2.3 \mathrm{kV} / \mathrm{mm})$, which is taken as the upper limit of the y-axis in Fig. 4.12. However, corona discharge and electro-jetting can already occur at lower field strengths, for example in the presence of dust particles or from a small curvature of the water mass collected on the target. From Peek's equation [107] and the radius of our target, we can calculate that the maximum working voltage will be about $25 \mathrm{kV}$ with a $2 \mathrm{~cm}$ distance between the guard ring and the target, which can explain the lower experimental efficiencies obtained in our experiments. On the other hand, some experimental data show higher efficiencies than the theoretical prediction, which is possibly due to the spread of the initial velocities of droplets compared to what was measured in Fig. 4.10. Variations in the droplet distances in the absence of an electrical field (which are related to the spread in the initial kinetic energy of the droplets) were also observed in initial experiments, and can be attributed to interaction with fluid agglomerated not the nozzle plate.

As can be seen from Fig. 4.12(a), increasing the pressure above 1.2 bar (in the $10 \mu \mathrm{m}$ diameter nozzles) will not contribute to a minimization of the viscous energy loss in stage 1. Decreasing the droplet flight distance helps to decrease the air drag loss, however this increases the required electrical field strength and increases the chance of electrical discharge (Fig. 4.12(b)). Induction of additional charges in the droplets can strongly decrease the required optimal electrical field. By using a larger nozzle diameter, the system energy loss will dramatically decrease as shown in Fig. 4.12(c). However, the charge density will decreases with $R^{-3 / 2}$, necessitating a significant increase of the required electrical field (voltage) on the target, thereby increasing the chance of electrical breakdown. As can be also be inferred from the figure, our experiments with a $12 \mu \mathrm{m}$ radius jet (from the $30 \mu \mathrm{m}$ diameter nozzles) obtained a lower efficiency than predicted ((maximal $48 \%$ vs. $70 \%$, resp.) as it exceeds the maximal practical operational electrical field (shown as the purple dashed line). Comparing the slope of efficiency and field strength for the three parameters that are varied in Fig. 4.12, the jet radius is the parameter that lends itself best for increasing the efficiency while keeping the cost of the required increase in field strength limited. 


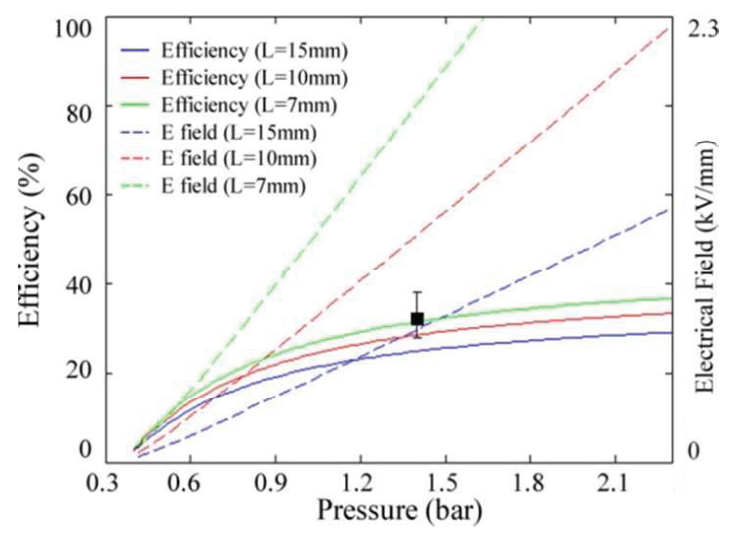

(a)

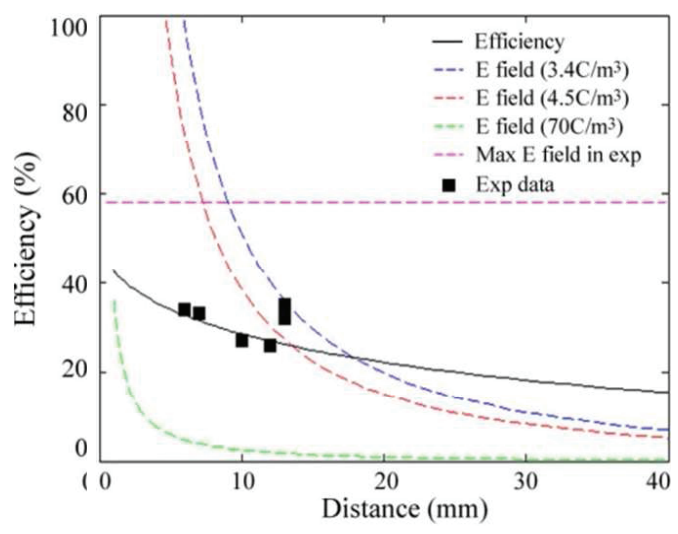

(b)

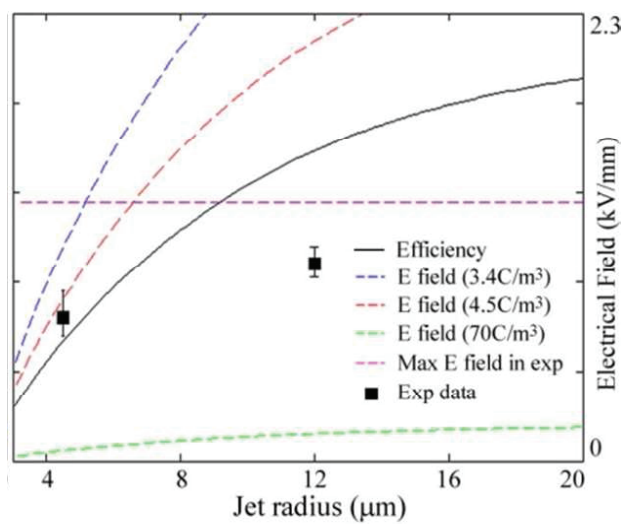

(c)

Figure 4.12: Maximum conversion efficiencies (solid lines, left y-axis) predicted with variations of applied pressure (a), nozzle-target distance (b) and jet radius (c) as well as optimal electrical field strengths (dashed lines, right $\mathrm{y}$-axis) required for the maximum conversion efficiency (solid lines). Purple dashed lines indicate the practical maximum electrical field with $1.3 \mathrm{kV} / \mathrm{mm}$. Parameters used in these graphs are droplet charge density $\rho_{e}=3.4 \mathrm{C} / \mathrm{m}^{3}$, applied pressure $\mathrm{p}=140 \mathrm{kPa}$, distance between guard ring and target $\mathrm{d}=10 \mathrm{~mm}$ and jet radius $\mathrm{R}=5 \mu \mathrm{m}$, when not stated otherwise. 


\subsection{Discussion}

Besides viscous loss and air drag as significant loss factors, as discussed, there are some other minor loss factors in the conversion process that will be discussed in this section. Several energy losses will occur due to the current transport through several resistances. The voltage needed for electrolysis of water (due to the charge transfer resistance and over-potential at the electrode) is about $2 \mathrm{~V}$, which must be overcome at the electrodes to provide the flow of current generated by the streaming current. Due to the use of the guard ring, the voltage at the location of droplet breakup will not be affected by the target potential. A small conduction current might still be generated in the jet, moving charge back into the reservoir. A decreased streaming current in the jet however will not affect the energy conversion efficiency in ballistic conversion, since the target voltage can always be sufficiently high to decelerate the droplets to zero velocity. Only if the required electrical field is more than $2.3 \mathrm{kV} / \mathrm{mm}$ or the required potential higher than $25 \mathrm{kV}$, electrical losses due to electro jetting or corona discharge will occur. Corona discharge or electro jetting from the target can play a significant role. The current losses from deflected droplets or the electro jetting current $\left(I_{3}\right)$ were measured in experiments. The power loss of electro jetting can be calculated as: $I_{g r} V_{g r}$, where $I_{g r}$ and $V_{g r}$ are the current and voltage on the guard ring. These losses should be avoided by building a mechanically well-defined and clean set-up and avoiding regimes with extreme electric field strengths, which can be effected by inducing a larger charge density on the droplets by applying a negative voltage to the guard ring.

We also found that the spread of droplet initial velocities in different experiments can result in energy loss. In this case either a lower potential is required to make all droplets land on target, or a loss of current must occur because some slower droplets are reflected. This energy loss is independent of the losses studied in this paper, and could be treated separately. From our measurements we estimate that at peak efficiency (with optimized target potential) approximately $10 \%$ of the droplets were reflected from the target, which yields that $90 \%$ of the current $I_{1}$ arrives on target and the system efficiency is reduced by approximately $20 \%\left(P_{\text {out }}=R_{\text {load }} * I_{2}^{2}\right)$. When the droplets consume nearly all kinetic energy before landing on target or when the droplets have high charge density, the straight train of droplets becomes cone-shaped probably due to the electrical repulsion between charged droplets, as in Fig. 4.5. This phenomenon will cause a trajectory change for the droplets at the inside and outside of the cone, resulting in much easier deflection of the droplets at the outside of the cone. In general, generating monodisperse droplets is recommended, to decrease repulsion between droplets reducing this loss factor. To minimize the liquid friction energy loss, it would be recommended to use a low viscosity liquid, which at the same time should have a low vapor pressure to prevent evaporation of charges during droplet transport. Polar solvents are recommended to use for the induction of charges 
in the droplets. Finally, solvents with a high surface tension are preferred since they have a higher Rayleigh limit.

\subsection{Conclusions}

We modeled and determined experimentally the energy losses during the conversion process from pressure energy to electrical energy. The energy conversion process is divided in two stages: 1) from pressure-driven input mechanical energy to kinetic energy of a charged droplet flow; 2) from kinetic energy of a charged droplet flow to electrical energy on the target. An experimental set-up was built to quantify the losses occurring in these two stages. To evaluate the loss factors in stage 1, we developed simulations to calculate the shear stress and obtained a general energy loss coefficient $K$ for different size nozzles. From the simulated velocity of the jet, we could also obtain the effective kinetic energy of droplets in the jet, calculate the flow rate and show the agreement with experimental results. To evaluate the loss factors in stage 2 a fitting model was developed to simulate air drag within a train of droplets. This model was extended to include the additional deceleration of droplets under application of an electrical field. Furthermore the entrainment of the surrounding air yields a lower air drag compared to single droplets. We modelled the air drag matching to the experimental data and numerical simulations. Combining the air drag model and the electrical force term with the optimum field strength we could estimate the loss factor in the conversion from kinetic energy to electrical energy. The loss terms of stage 1 and stage 2 were combined into an efficiency model as a function of the applied pressure, nozzle-target distance, jet radius and charge density. From the efficiency model it was estimated that increasing the nozzle size is the most suitable option to increase efficiency, and that decreasing the nozzle-target distance and increasing the applied pressure are less beneficial because of the requirement of a greater increase in field strength, which may lead to electrical discharge. Thus, it is recommended to fabricate a larger size nozzle, fit to to minimize the energy loss in the proposed ballistic conversion system. 


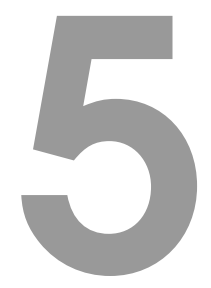

\section{Velocity profile inside piezo-acoustic inkjet droplets in flight. *}

Inkjet printing deposits droplets with a well-controlled narrow size distribution. This paper aims at improving experimental and numerical methods for the optimization of drop formation. We introduce a method to extract the one-dimensional velocity profile inside a single droplet during drop formation. We use a novel experimental approach to capture two detailed images of the very same droplet with a small time delay. The one-dimensional velocity within the droplet is resolved by accurately determining the volume distribution of the droplet. We compare the obtained velocity profiles to a numerical simulation based on the slender jet approximation of the Navier-Stokes equation and we find very good agreement.

\subsection{Introduction}

Inkjet printing is well known for its impressive reproducibility of the drop formation [108-110]. This has made inkjet printing technology a reliable technique for drop deposition of liquids for a broad range of applications [111, 112], owing to the increase of the deposition accuracy and further reduction of the droplet sizes [113, 114].

*Published as: Arjan van der Bos, Mark-Jan van der Meulen, Theo Driessen, Marc van den Berg, Hans Reinten, Herman Wijshoff, Michel Versluis and Detlef Lohse, Velocity Profile inside Piezoacoustic Inkjet Droplets in Flight: Comparison between Experiment and Numerical Simulation, Phys. Rev. Applied, 1, 014004. (2014) 
Numerical models for inkjet printing, e.g., finite element, finite difference, and boundary integral methods, are used to develop printheads for these applications. One very successful model is the slender jet approximation of the Navier-Stokes equation, often also denoted as lubrication approximation. It has been shown to be fast and reliable for modeling of drops $[115,116]$ and sprays [117] and incorporates breakup dynamics and coalescence of the drops [12]. The low-CPU time requirements of such a model, which are of the order of minutes on a personal computer, allows for rapid exploration of a large parameter space in a short time.

As a result of high droplet velocities and decreasing droplet volumes, the experimental validation of the numerical models becomes increasingly challenging [108, $110,118]$, as a result of decreasing droplet volumes and high droplet velocities. Visualizing fast inkjet droplets requires ultra-high-speed cameras imaging at frame rates exceeding one million frames per second [85, 117, 119, 120], or stroboscopic techniques with very short illumination times $(<20 \mathrm{~ns})$ to freeze the motion in the droplets $[108,109,121]$. Even then the results are mostly limited to global estimates such as the overall drop velocity, drop volume, and number of satellites. In contrast, numerical models reveal detailed local information on tail formation, velocity development, and fluid dynamics throughout the entire drop formation.

In this chapter we present an experimental method that reaches beyond current ones; we will extract the local and global droplet dynamics during the entire formation of a single picoliter sized droplet. We introduce an advanced imaging technique that provides two snapshot images of the very same droplet with extremely high temporal and spatial resolution. The image pair is analyzed by extracting the contour of the droplet, and subsequently by calculating the volume distribution over the droplet. The one-dimensional velocity inside the droplet is given by the small displacements in the volume distribution over the time between the two recordings.

Our method allows for a fully quantitative comparison of the measured drop dynamics with the numerical models. Here we compare the experimental data with the results obtained from the lubrication approximation. The volume and velocity distribution obtained from experiment are used as initial condition for the numerical simulation. The time evolution of the droplet can then be used to validate both the experimental and numerical approach.

\subsection{Experimental setup}

Figure 5.1 shows a schematic overview of the experimental setup, which consists of essentially four components: printhead, light source, imaging system, and timing control hardware.

The inkjet printhead is developed by Océ Technologies [122-125]. The piezo inkjet printhead contains 256 similar ink channels with an inverted trumpet shape 


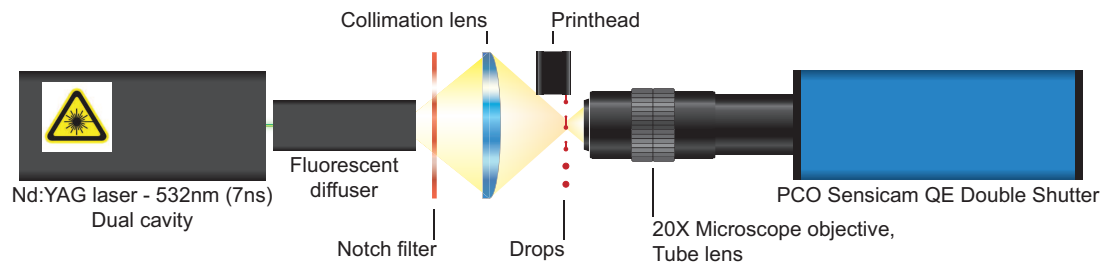

Figure 5.1: In the experimental setup the laser pulse from the Nd:YAG laser illuminates the diffusor, which emits a fluorescence light pulse of $8 \mathrm{~ns}$ at high intensity. The fluorescence is focused by an aspherical condenser lens onto the droplets. A notch filter, placed between the condenser lens and the droplets, prevents any remaining laser light from reaching the CCD camera. The images are recorded using an inline assembly microscope with a dual frame camera.

nozzle with an opening radius of $15 \mu \mathrm{m}$. The printhead is distinguished by a very reproducible drop formation $[110,122]$ and negligible angular distortion with respect to jetting direction. Applying an actuation pulse of $30 \mathrm{~V}$ to the piezoelectric actuator results in $11 \mathrm{pL}$ droplets with a final velocity of $4 \mathrm{~m} / \mathrm{s}$. Much higher velocities, up to $15 \mathrm{~m} / \mathrm{s}$, occur during the formation of the droplet at the meniscus and in the tail of the droplet.

The experiments were conducted with silicone oil, which has several advantages. First, the temperature dependence of the viscosity is less than $1 \% / \mathrm{K}$ and silicone oil therefore acts as a Newtonian liquid. Second, the surface tension of silicone oil is not easily affected by contamination, hence the surface tension can be assumed to be constant during the drop formation. The silicone oil that was used is AK10 of Wacker-Chemie $\mathrm{GmbH}$, which has a viscosity of $9.3 \mathrm{mPa}$ s, a surface tension of $20.2 \mathrm{mN} / \mathrm{m}$, and a density of $930 \mathrm{~kg} / \mathrm{m}^{3}$.

To illuminate the droplets, we use a dual-cavity Nd:YAG laser $(\lambda=532 \mathrm{~nm}$, Litron Nano-S). The laser creates a $7 \mathrm{~ns}$ laser pulse with high intensity $(65 \mathrm{~mJ}$ per pulse) and is coupled into a fluorescent diffuser. The fluorescent light generated in the diffuser remains short (approximately $8 \mathrm{~ns}$ ) and intense, while both the temporal and spatial coherence have been lost $([10,126,127])$. This makes it highly suitable for imaging purposes without speckle and interference fringes. The residual laser light, which could distort the image or even damage the CCD sensor, is removed with a notch filter. To ensure optimal coupling into the imaging system, the fluorescent light is collimated with an aspherical lens such that the numerical aperture closely matches that of the microscope $(\mathrm{NA}=0.4)$ [128].

To image the picoliter sized droplets a microscope is connected to a dual-frame PIV camera (PCO Sensicam QE Double Shutter), capable of capturing two consecutive images with a delay as low as $500 \mathrm{~ns}$ in between. The microscope is an inline assembly microscope (InfiniTube) equipped with a $20 \times$ objective with a NA of 0.42 


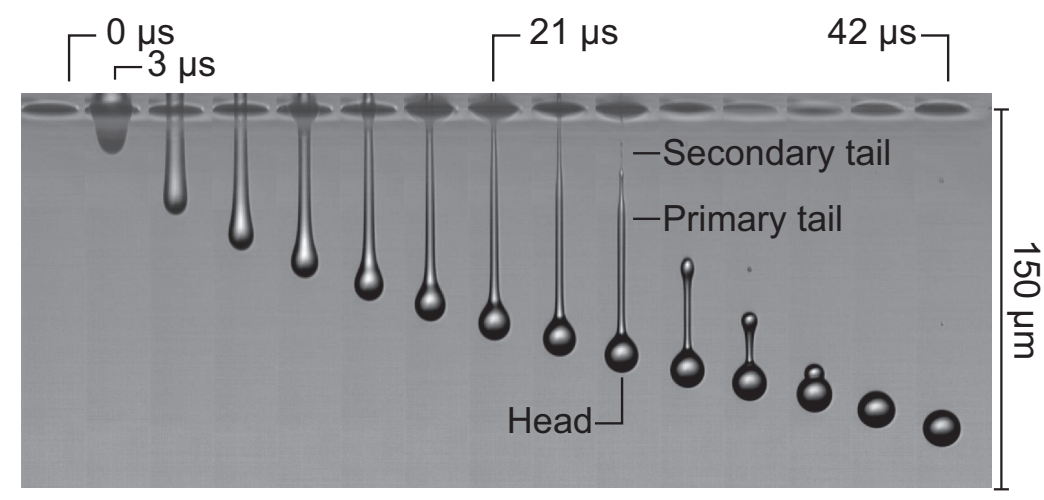

Figure 5.2: Time series of droplets recorded with single-flash photography. From left to right, multiple images of single droplets with a delay of $3 \mu$ s between these individual droplets. The width of the droplet is $23 \mu \mathrm{m}$, the tail is about $4 \mu \mathrm{m}$, and the secondary tail has a width below $1 \mu \mathrm{m}$. The figure illustrates the imaging quality of the setup, and the absence of motion blur due to the use of the $8 \mathrm{~ns}$ iLIF.

(Edmund Optics, M-Plan-APO). This results in an imaging resolution of approximately $365 \mathrm{~nm} /$ pixel. Because of the imperfection of the optics the resolution is not exactly constant over the whole field of view. A correction for this "pincushion" has been applied to the images. This correction is determined using a highly accurate calibration grid. Figure 5.2 shows a time series of drop formation, illustrating the typical high-quality images obtained with our system.

The timing is controlled by a high-precision delay generator (BNC 575, Berkeley Nucleonics) which triggers the camera, laser, and printhead. The illumination time and the delay between two flashes is verified using a high-speed photodetector (Thorlabs DET 210) which has a typical rise and fall time of $1.2 \mathrm{~ns}$.

\subsection{Experimental methods}

\subsubsection{High-resolution imaging of drop formation in flight}

Imaging drop formation in inkjet printing with high detail is very challenging, as the micron-sized droplets move with a velocity of several meters per second. It requires a high degree of spatial and temporal resolution, which is offered by a combination of large high optical quality imaging systems with sensitive high-resolution cameras. It is convenient to use a camera with a small pixel size to keep the magnification to a minimum (as it may severely distort the image quality).

Equally important is the time duration that the CCD sensor is exposed. This time duration can be the exposure time of the camera or, in case of a short flash, the 
duration of the flash. The exposure needs to be optimized such that sufficient contrast is obtained while minimizing the motion blur. The following criterion (Ref [10]) is used for the maximum allowable exposure time $(\tau)$ :

$$
\tau \leq \frac{\text { pixel size }}{u \cdot M_{\text {eff }}}
$$

where $u$ is the velocity of the droplet. The spatial resolution of the images is given by the magnification of the optics $\left(M_{e f f}\right)$ and the pixel size of the CCD sensor. In our experiment the fastest fluid element is displaced with a velocity of $15 \mathrm{~m} / \mathrm{s}$. Following equation (5.1) the maximal allowable illumination time is $18 \mathrm{~ns}$. Here we use a flash of approximately $8 \mathrm{~ns}$, thus for the present setup the motion blur is reduced to less than half the size of a pixel.

\subsubsection{Dual imaging of drop formation in flight}

As inkjet printing is very reproducible, stroboscopic imaging or single flash imaging would suffice for a statistical analysis. Here the aim is to visualize the dynamics during drop formation, for which the reproducibility is not sufficient; this requires at least two images of the very same droplet. Although it may seem straightforward to use time-resolved high-speed imaging techniques, the time and length scales in this problem would require ultra-high-speed imaging systems running at several million frames per second, e.g., the Brandaris Brandaris 128 camera [85, 129], with the added complexity of optical configuration, triggering and illumination.

Our approach creates a time series of the entire drop formation by shifting the delay of the flash with respect to the start of drop formation. As a result of the high degree of reproducibility of the inkjet system, the result of such a stroboscopic technique is very similar to a time series obtained in time-resolved high-speed imaging $[85,120]$. Here we add functionality by recording two consecutive images of one single droplet at each time step with the double shutter camera and the dual-cavity laser. It is key to have the time delay $\left(\tau_{f}\right)$ between the two consecutive images, denoted as frame A and frame B, such that the lowest possible detectable displacement is obtained. The delay is limited in two ways. Its lower limit is given by hardware limitations of the camera, here $500 \mathrm{~ns}$. The upper limit comes from a fluid dynamical point of view. For the determination of the velocity along the axis of symmetry of the droplet, we assume that the motion is dominated by the inertia of the fluid. To meet this assumption, $\tau_{f}$ must be smaller than the viscocapillary time scale $\left(\tau_{v c}\right)$, given by

$$
\tau_{v c}=\frac{\mu R}{\sigma} .
$$

In the experiments we use silicone oil with a viscosity of $9.3 \mathrm{mPas}$, and a surface tension of $20.2 \mathrm{mN} / \mathrm{m}$. As the tail behind the head of the droplet has a width of approximately $10 \mu \mathrm{m}$, giving an upper limit of $\tau_{v c}$ of approximately $2 \mu \mathrm{s}$. 

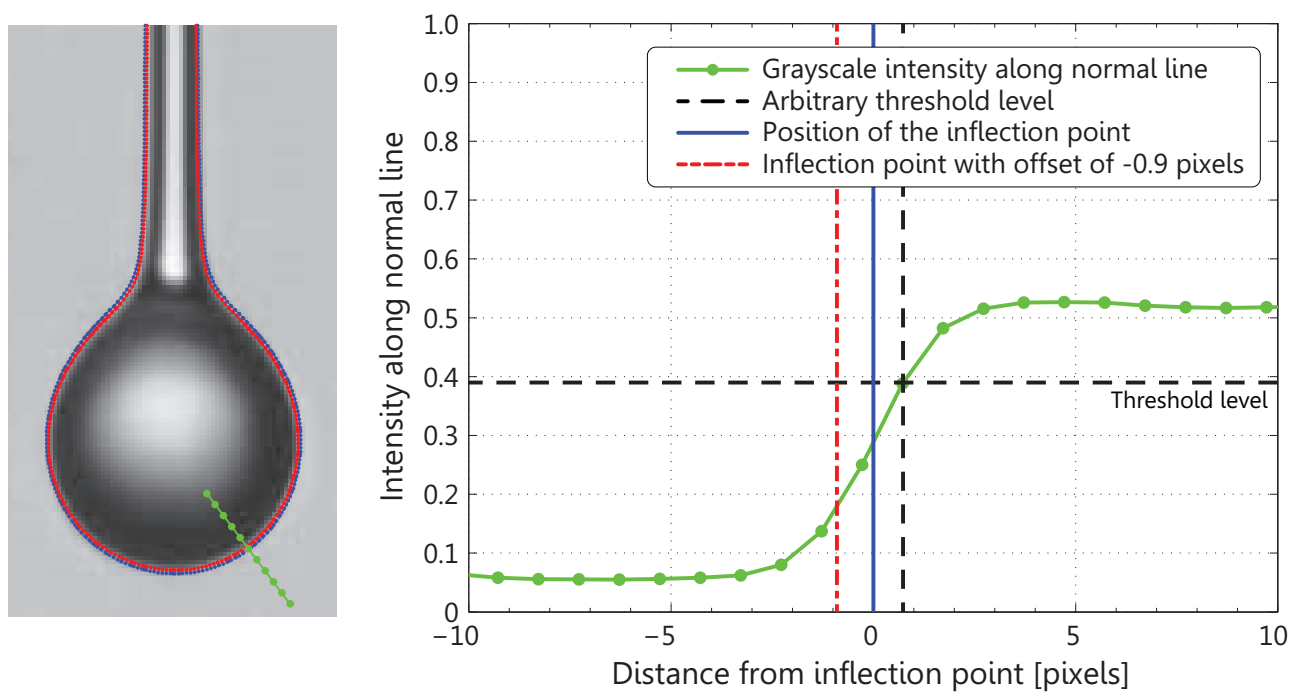

Figure 5.3: Accurate subpixel edge detection, independent of the contrast in the image, is required. On the left a region of interest of the drop image is shown. First, the edge is determined using a standard threshold method (data not shown). From this trace the normal lines along the boundary of the drop are calculated (dotted green line). By calculating the inflection point along the normal lines the intensity independent contour is determined (blue). The red line shows the corrected position of the surface, which is determined by volume conservation arguments, see section 5.5. The right part shows the intensity curve along the normal line (green dotted line).

\subsection{Image processing}

The experimental setup delivers detailed high-resolution images, from which the volume distribution of the droplet is determined. It requires accurate subpixel edge detection, independent of the contrast of the image. Part of the printhead blocks the illumination, causing an uneven intensity distribution in the images. In addition, the intensity of the light source fluctuates approximately $4 \%$. With standard (fast) edge detection techniques the precise location of the edge is very sensitive to these fluctuations. This problem is solved by using a two-pass edge detection method, illustrated in Fig. 5.3. The first pass consists of a conventional contour tracing routine (Mathworks Matlab). The second pass determines the intensity curve along the normal of the contour. By calculating the inflection point [130] along these normal lines the intensity independent contour is found.

To verify the validity of this method, a spherical droplet is imaged at a constant delay $\left(t_{d}\right)$ while gradually increasing the background intensity of the image within the dynamic range of the camera. From each of the images the radius of the droplet is 


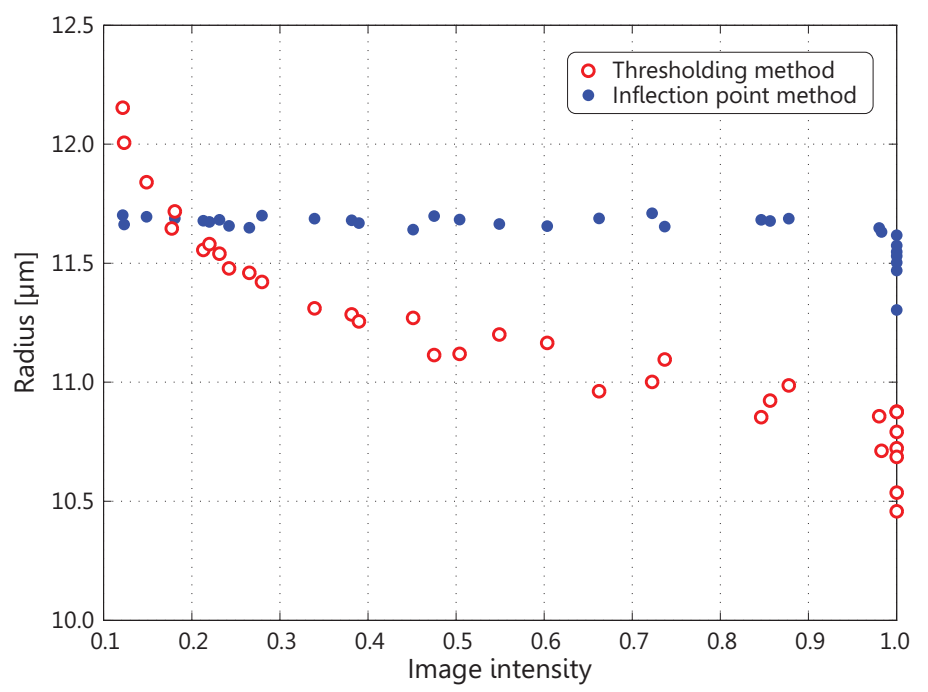

Figure 5.4: A spherical droplet is imaged while gradually increasing the intensity of the illumination over the dynamic range of the camera. The radius is determined both using a thresholding method (red open dots) and by determining the inflection point along the normal lines (blue filled dots). The radii from the threshold images change by $20 \%$ for increasing intensity, while the proposed method shows less than $1 \%$ variation. The remaining variation is primarily caused by the residual variation in the reproducibility of the drop formation (as shown in the inset of Fig. 5.6)

calculated using the proposed method. Figure 5.4 shows the drop radius for increasing background intensity. This shows that, while the conventional method proves unreliable, our proposed method performs very well over the entire dynamic range of the camera. Only when the background is overexposed the method fails. This method allows for an accurate intensity independent contour determination.

\subsection{Droplet volume}

From the contours of the droplet the volume of this droplet can be determined, assuming axial symmetry around the central axis of the droplet. Here we define the radius as the perpendicular distance between the central axis and the edge of the droplet, and is given by $R\left(x, t_{d}\right)$, where $x$ is the axis of symmetry, and $t_{d}$ is the time with respect to the start of the drop formation (Fig. 5.5). The volume of a droplet is calculated by integration over $x$,

$$
V_{\text {drop }}\left(t_{d}\right)=\pi \int_{x_{0}}^{x_{t i p}} R\left(x, t_{d}\right)^{2} d x .
$$




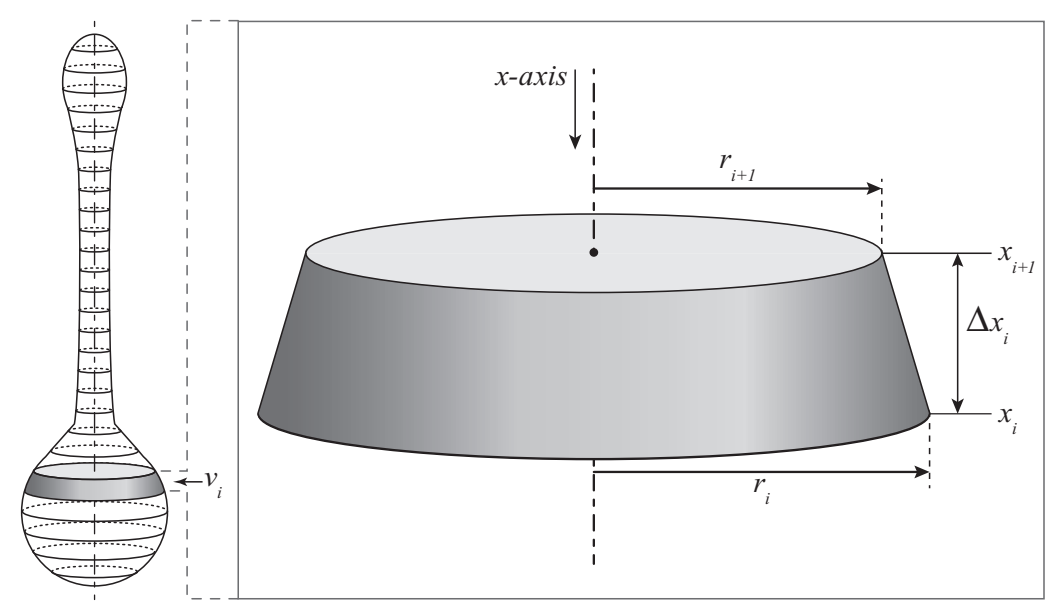

Figure 5.5: For each droplet the contour was determined with subpixel accuracy. To calculate the volume distribution the drop is assumed to be axisymmetric. The volume $\left(v_{i}\right)$ of element $\mathrm{i}$ of the drop is estimated by truncated cones with radii $r_{i}$ and $r_{i+1}$ and a height $\Delta x_{i}$.

Here $x_{0}$ is the nozzle position and $x_{t i p}$ the tip of the drop. Because of the subpixel edge detection, the discrete $\mathrm{x}$ values were resampled into nonequidistant values. The sampling resolution $\mathrm{dx}$ has become so small that we can safely assume that the radius varies linearly over $\mathrm{dx}$. Hence, the volume of each element becomes a truncated cone with a finite width of $\Delta x_{i}$ (illustrated in Fig. 5.5), where each cone has a volume

$$
v_{i}=\pi \frac{\left(r_{i}^{2}+r_{i+1}^{2}+r_{i} r_{i+1}\right)}{3} \Delta x_{i}
$$

This gives the total drop volume at time $t$,

$$
V_{\text {drop }}\left(t_{d}\right)=\pi \sum_{i=0}^{i=N} \frac{\left(r_{i}^{2}+r_{i+1}^{2}+r_{i} r_{i+1}\right)}{3} \Delta x_{i}
$$

This method is used to determine the volumes of all droplets in a time series, as shown in Fig. 5.6. The figure shows how the volume increases as the droplet emerges at $16 \mu \mathrm{s}$ from the nozzle. At $60 \mu \mathrm{s}$ the droplet detaches from the meniscus, after which, for increasing delay, the shape of the detached droplet changes from elongated to spherical. The blue data show the volume, assuming the physical edge at the inflection point, as described in the previous section. The figure shows that for this data the volume increases over time. As evaporation is negligible and the drop formation is extremely reproducible, the actual volume of the detached droplet must remain constant. The origin of this overestimation lies in the physical surface 
not being at the inflection point. To compensate for this inaccuracy, an inward shift of $330 \mathrm{~nm}$, with respect to the inflection point along the normal is introduced to the edge detection method. Figure 5.6 (red data) confirms the accomplishment of the correction, as the volume remains constant over time. To confirm that the stability is time independent, the figure displays two full drop evolutions, recorded at different times.

By displaying the volume in frame A against the volume in frame B over a time series (inset in Fig. 5.6), it is possible to estimate both the accuracy of the method and the reproducibility of drop formation. The droplet reproducibility is $V_{0}=11.02 \pm 0.13 \mathrm{pL}$. For a single drop the perpendicular distance from the linear regression line is

$$
\Delta V_{\text {drop }}\left(t_{d}\right)=\frac{V_{\text {drop }}\left(t_{d}\right)_{A}-V_{\text {drop }}\left(t_{d}\right)_{B}}{\sqrt{2}} .
$$

Here $V_{\text {drop }}\left(t_{d}\right)_{A}$ is the drop volume for frame $\mathrm{A}$, and $V_{\text {drop }}\left(t_{d}\right)_{B}$ is the drop volume of the same droplet in frame B. By calculating three times the standard deviation $(3 \sigma)$ over all volumes we find the accuracy of the method, here $3 \sigma=17 \mathrm{fL}$ ). 


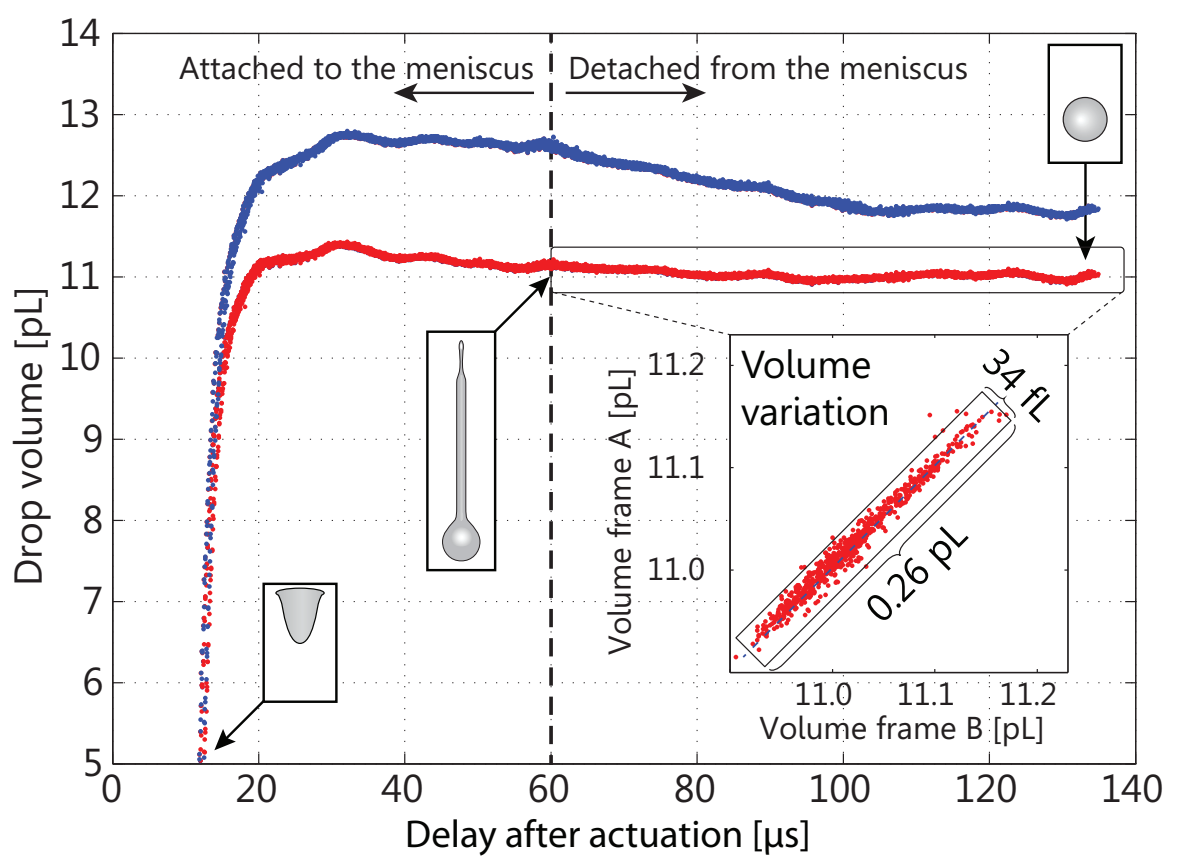

Figure 5.6: The total volume $V_{\text {drop }}$ of each ejected droplet, as extracted from the recordings, given as function of the delay time. For each delay the information of two image pairs, i.e. four images, are used. The second pair is recorded one full period after the first to confirm stability at longer timescale. After a delay of $10 \mu \mathrm{s}$ the meniscus starts moving outward, and at approximately $60 \mu$ s the droplets are detached from the meniscus. The blue data gives the uncorrected volume following the inflection point method, while the red point gives volume after correcting using volume conservation. The inlet shows the calculated volume in frame A against the volume in frame B, which illustrates the volume reproducibility $\left(V_{\text {drop }}=11.02 \pm 0.13 \mathrm{pL}\right)$ and the accuracy of the $\operatorname{method}(3 \sigma=17 \mathrm{fL})$. 


\subsection{Droplet velocity}

To determine the velocity within the droplet at time $t_{d}$ the displacement of the volume elements $\left(v_{i}\right)$ is determined with a Lagrangian method. An image pair is captured for each single droplet; frame A at a drop delay $t_{d}$ and frame B at $t_{d}+\tau_{f}$. Here the time difference between the two frames $\left(\tau_{f}\right)$ was set to $600 \mathrm{~ns}$. First, the volume distribution is determined for both frames individually. Then, starting at the tip of the drop, the volume of each element in frame $\mathrm{A}$ is mapped to the volume elements in frame B (illustrated in Fig. 5.7). Here we define $\bar{x}_{A}(i)$ as the center of mass of the volume element in frame $\mathrm{A}$, and $\bar{x}_{B}(i)$ as its mapped counterpart. The velocity is subsequently determined by calculating the displacement of the center of mass of each element,

$$
U_{i}=\frac{\bar{x}_{A}(i)-\bar{x}_{B}(i)}{\tau_{f}} .
$$

Thus, for a given delay $t_{d}$, this method reveals the average velocity $U_{i}$ of each element $\bar{x}_{A}(i)$ throughout the droplet. This is illustrated in Fig. 5.8, where the left part shows the image pair, the middle part shows the volume of the droplets in the image pair, and the right part shows the calculated velocity. 


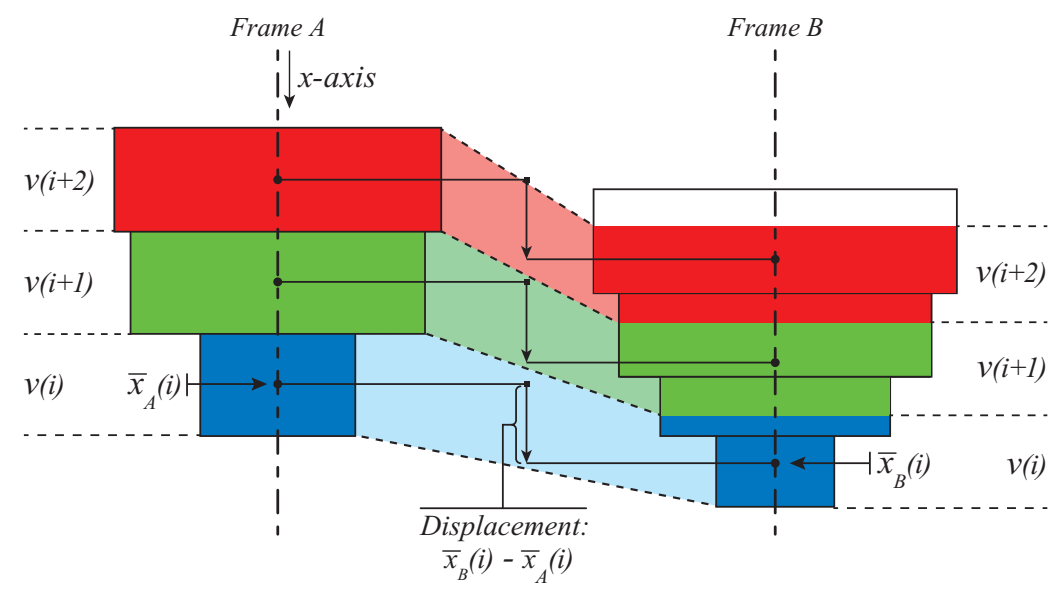

Figure 5.7: The volume distribution is calculated for frames A and B. The volume per element of the first time step is mapped to the volume elements in the second time step. The displacement of the corresponding center of masses divided by the time interval between the recordings gives the mean velocity of the volume elements.

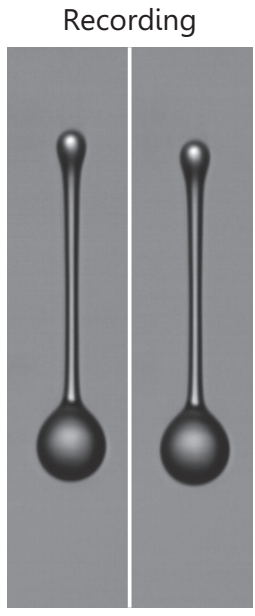

Frame A Frame B
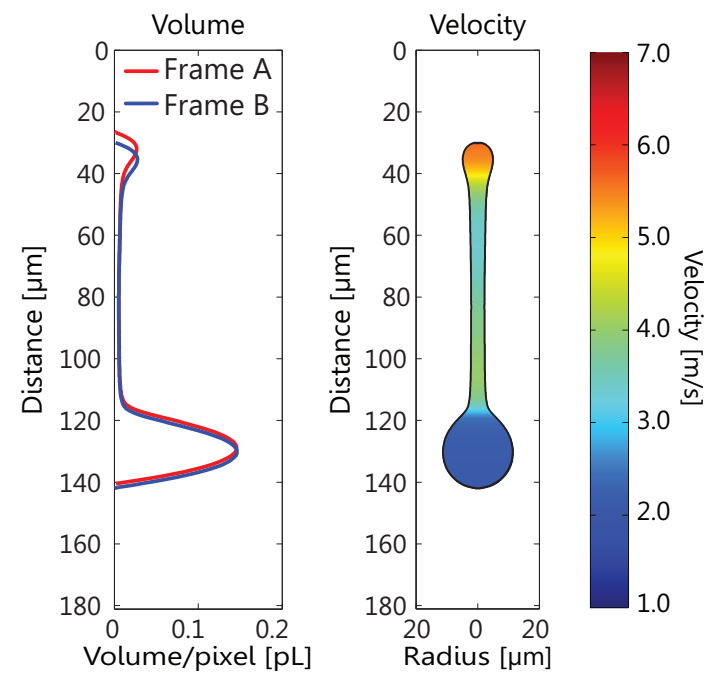

Figure 5.8: The different steps in the experimental procedure. Left: First two recordings of the same droplet are made, with a delay of $600 \mathrm{~ns}$ between the two frames. Middle: For both images the volume distribution over the droplet is calculated with sub-pixel accuracy. Right: The velocity inside the droplet is determined by calculating the displacement of the volume elements. The velocity is represented by the color scale. 


\subsection{Validation of the experimental methods}

With the velocity and volume distribution known at any given time, we can also simulate the drop formation numerically. We use the velocity and volume distribution obtained from the experiment as initial condition for the numerical simulation. The drop formation is simulated in a lubrication approximation (also known as slender jet approximation); a systematic reduction of the Navier-Stokes equations, based on the slenderness of the liquid jet. It has been shown that this approximation gives accurate results for the evolution of a slender jet [12, 115, 117, 118, 131, 132]. Here the previously developed discretization model by Driessen \& Jeurissen [12] is used. In this discretization model the singularities that occur at pinch-off and coalescence are removed by a adding a regularization to the surface tension term. As the regularization scales with the detail of the simulation, its influence vanishes in the limit of an infinitesimally small spatial step size. The model returns the shape, volume distribution, and the average axial velocity of the droplet, similar as we extract from the experiments.

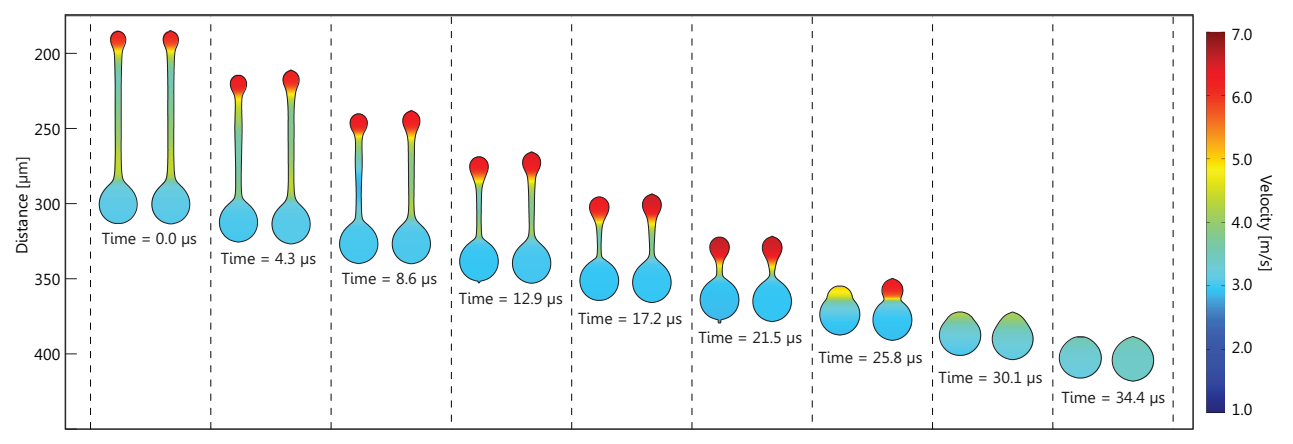

Figure 5.9: Comparison between the experimental and numerical result. At different times, we show both the experimental result (left) and the numerical result (right). The experimental result is obtained from a different droplet each time, whereas the numerical droplet is calculated from the initial volume and velocity distributions from the experiment shown at $\mathrm{t}=0 \mu \mathrm{s}$. The velocity is represented by the color scale.

For the validation two different cases were used. The first case is the evolution of a single droplet under normal jetting conditions, ejected at a drop on demand frequency of $10 \mathrm{kHz}$. As initial condition for the start of the numerical simulation, we use the experimentally obtained velocity and volume distribution after the droplet has detachment from the meniscus. Additionally, the density, viscosity, and surface tension of the silicone oil were used as input parameters. Figure 5.9 shows the evolution of the droplet for the experimental method and the numerical simulation, where we 


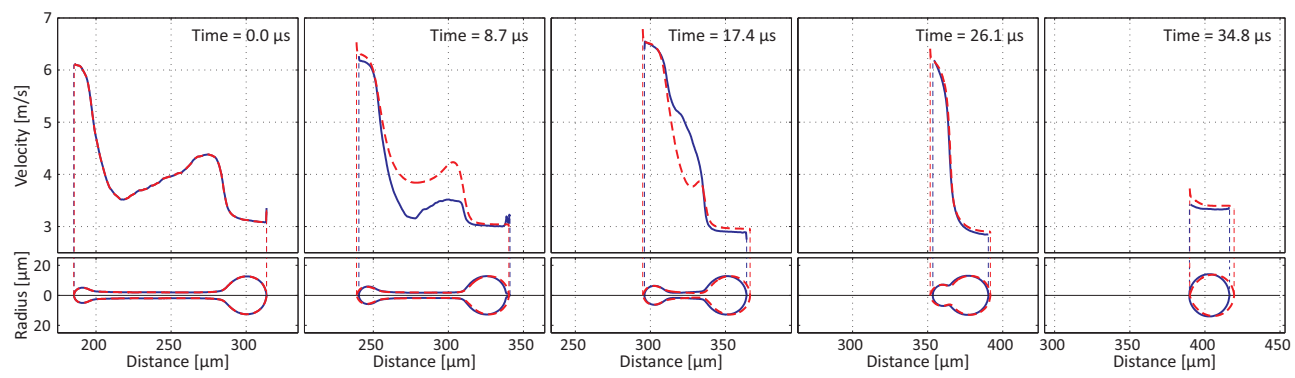

Figure 5.10: Detailed comparison between the experimental result (blue) and the numerical result (dashed red) at five times instances. The top row shows the velocity distribution, whereas the bottom row shows the profiles. The experimental and the numerical profiles are nearly identical. The Rayleigh-Plateau instability causes the velocity inside the tail to vary from droplet to droplet (also visible in Fig. 5.11). During the contraction of the droplet there is very good agreement between the velocities of the head and tail droplet.

find excellent agreement. The connecting filament between tail and head remains stable during the entire contraction, i.e., it doesn't break up due to capillary forcing. Furthermore, the quantitative agreement of both magnitude and distribution of the velocity are very good during the full dynamical process.

To compare the results in more detail Fig. 5.10 shows the velocity and radius at five different times. For each time step we show the experimental result (solid blue line) and the numerical result (dashed red line). The experimental and the numerical profiles are nearly identical. However, there is a small distortion in the velocity in the tail of the droplet over the different experimental results. As the experimental results are obtained from a different droplet each time, the Rayleigh-Plateau instability causes the velocity inside the tail to vary from drop to drop. To support this claim, we show the experimental data of the stroboscopic recording with a high temporal resolution in Fig. 5.11. It shows the momentum distribution over the droplet for all drops in the experiment (top) as compared to the numerical simulation (bottom). Here it can be observed that the perturbations on the tail of the droplet are uncorrelated between the different droplets in the experiment.

To demonstrate that the method to determine the velocity also works for separated liquid bodies, we apply it to a second case. Here two droplets are jetted from the same nozzle with a short time delay. The trailing droplet is ejected with a higher velocity, causing the two droplets to collide at approximately $350 \mu \mathrm{m}$ downstream from the nozzle. Figure 5.12 shows the experimental and the numerical results side by side, and again we obtain very good agreement. Again we observe small erratic behavior in the experiments. The momentum distribution as a function of time, displayed in 


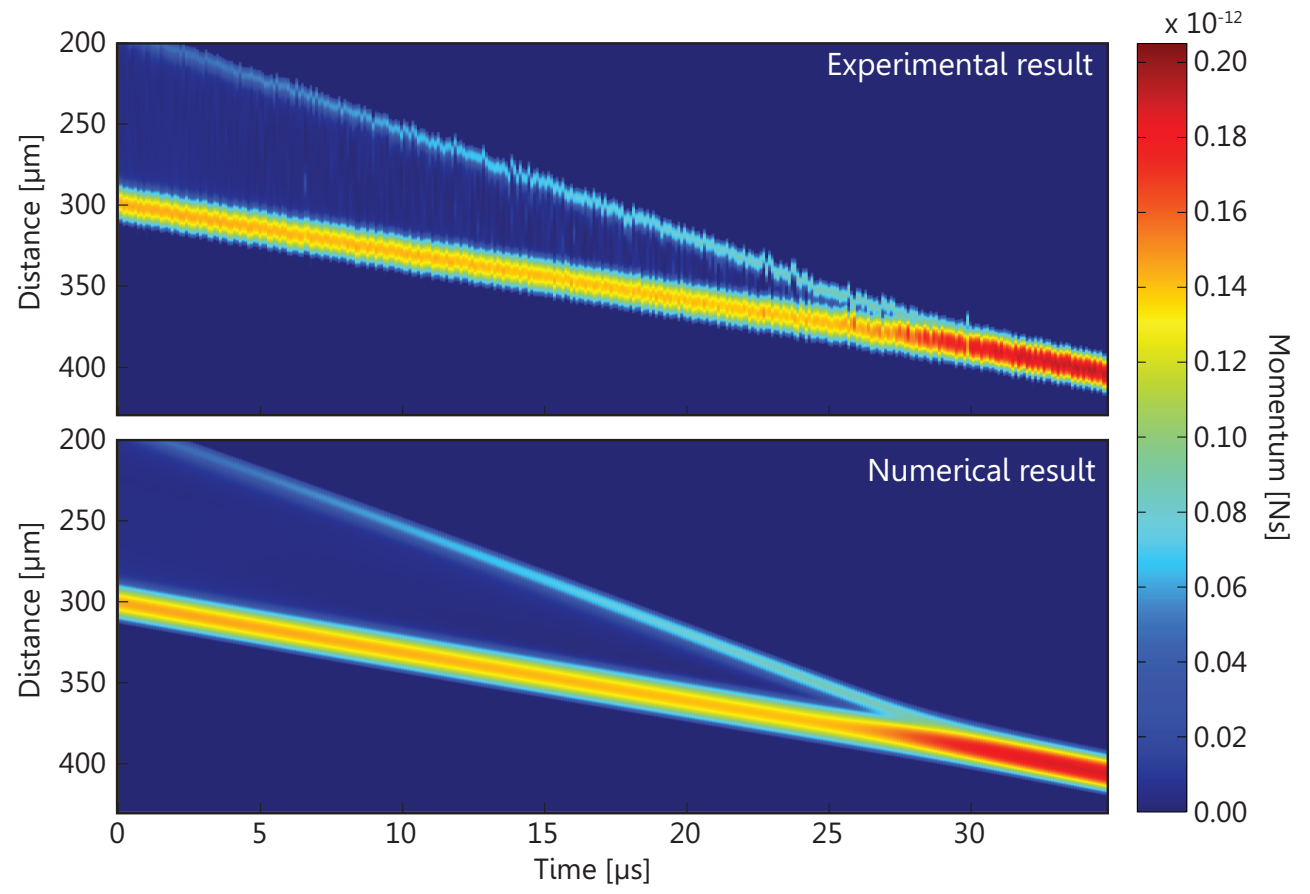

Figure 5.11: The momentum distribution over the droplet for all drops in the experiment (top) and the numerical simulation (bottom). In the experiment there is a delay of $100 \mathrm{~ns}$ between each subsequent droplet. In the experimental result it can be seen that the position of the tail and the momentum in the tail vary slightly between different droplets due to the Rayleigh breakup in the tail.

figure 5.13, shows these disturbances more clearly. The jetting reproducibility for the case of two colliding droplets is lower than for the former case with a single droplet (Fig. 5.11). Still, even for this second case the overall agreement is very good. 


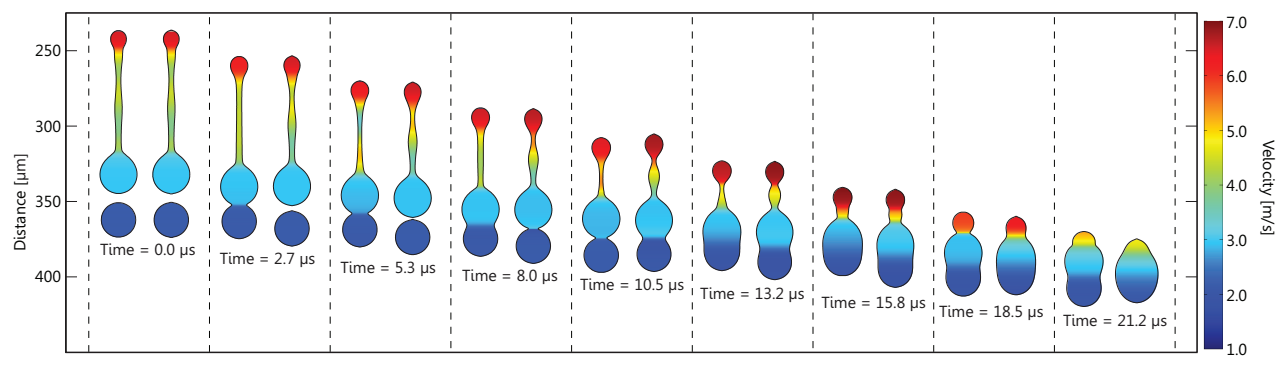

Figure 5.12: Comparison between the experimental and numerical result for a case of colliding droplets. We show the experimental evolution (left) and the numerical results (right) at different times. The numerical droplet evolution is calculated from the experimental volume and velocity distributions at $\mathrm{t}=0 \mu \mathrm{s}$. The color scale represents the velocity.

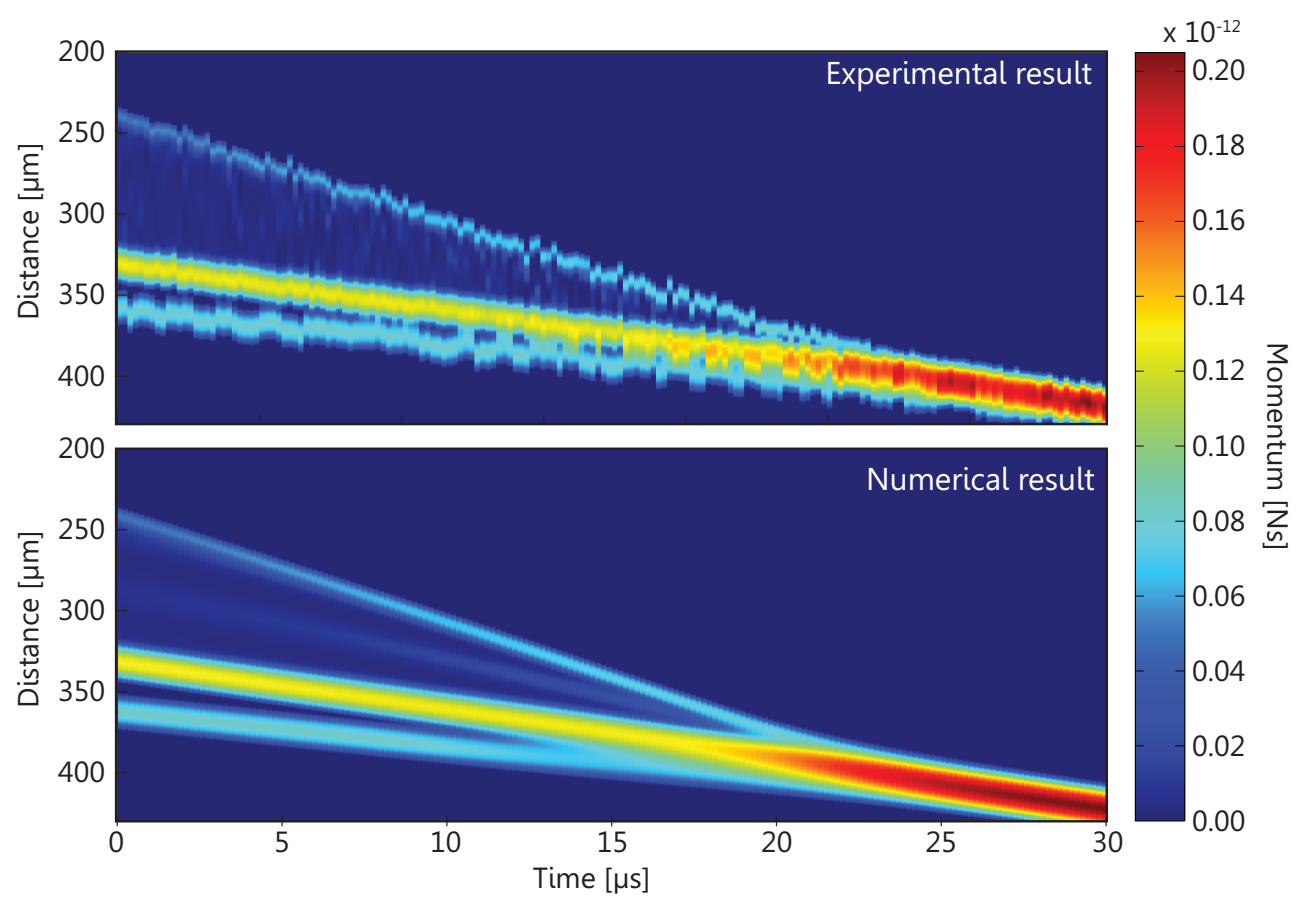

Figure 5.13: The momentum distribution for the colliding droplets in experiment (top) and numerical simulation (bottom) showing overall good agreement but with an apparent lower experimental reproducibility in droplet behavior. 


\subsection{Conclusion \& Outlook}

This work presents a novel method for quantitative analysis of the internal flow of drop formation in flight. Droplets are imaged using laser-induced fluorescence illumination, with a very short illumination time, allowing for detailed images without motion blur. Two images of a single droplet are imaged by using a dual frame setup. The images are analyzed and the volume is calculated. Here we find that the reproducibility of inkjet printing is approximately $0.13 \mathrm{pL}$ and an accuracy in the volume estimate approximately $17 \mathrm{fL}$. The reproducibility is limited and, to accurately extract the droplet dynamics, at least two images of a single droplet are required. Here we demonstrate a method to extract the one dimensional velocity inside the droplet, and validate the method with a numerical model. We find excellent agreement, confirming the applicability of the method.

The presented method is intended to study drop formation, in order to verify numerical methods, and to increase numerical utilization. This method may be extended towards drop formation in jetting of complex fluids with non-Newtonian rheology, quantitatively extracting non-Newtonian parameters from such a comparison.

With two recordings of the same droplet, it is only possible to measure its position and velocity. Technically it would be feasible to expand the setup, such that a series of images of a single droplet is captured at high spatial and temporal resolution. A third recording of the same droplet would in principle suffice to extract also the acceleration of the fluid. From the acceleration and the contour of the droplet, the forces that act on the liquid can be calculated. The capillary forces can be calculated from the droplet curvature, and therefore the local viscosity can be obtained. Thus, this method can be used as an extensometer to measure non-Newtonian properties and ink rheology at high speeds and high stretch rates encountered during inkjet drop formation. 


\section{6}

\section{Drop formation in piezo acoustic inkjet printing: Benchmarking CFD against experiment *}

Computational Fluid Dynamics (CFD) simulations are an important contribution to inkjet research, since the drop formation can be predicted for a range of acoustic and fluidic parameters. Numerous numerical methods and codes have been developed to predict the drop formation process. To test the predictions of these models, usually drop benchmarks are performed in which the models are compared to one another, without a priori knowledge of the expected formation behavior. In this work we make use of a previously developed experimental technique to measure the radius and velocity profiles of microdrops in flight. The data allows for a detailed comparison of the predictions of the numerical droplet formation models, by using measured drop profiles as input to the models and by comparing the predictions in subsequent time steps with the corresponding experimental recordings. We use two different droplet formation cases, capturing important features of the drop formation such as moment of contraction, drop velocity, and droplet size dispersion, to compare three numerical codes, namely Gerris, Fluent and the slender jet approximation of the Navier-Stokes (NS) equations.

${ }^{*}$ To be submitted as: Mark-Jan van der Meulen, Herman Wijshoff, Hans Reinten, Michel Versluis and Detlef Lohse, Drop formation in piezo acoustic inkjet printing: Benchmarking CFD against experiment. 


\subsection{Introduction}

The drop formation process is the main process in inkjet printing. Once a fluid volume is ejected from the printhead nozzle, the shape of the drops is only determined by internal drop forces, such as inertial forces, surface tension, and viscous forces, and external forces e.g. air drag forces and lubrication pressure from compressed air between colliding drops which can cause bouncing of the drops [133]. Electrokinetic forces are not considered in in this study. These forces can cause the drops to coalesce into a single spherical drop or to break-up into many satellite drops. These small satellite drops land on arbitrary places at the substrate, depreciating the quality of the final product. The final drop size and velocity are key parameters for the impact dynamics on the substrate, e.g. the spreading of the drops on the substrate[134] and air bubbles entrapped under the drop[135, 136]. The first front of the DOD inkjet droplets expelled from the nozzle are referred to as the head drop, the rest the tail. The velocity of the head drops and the total drop volume can directly be controlled by the actuation amplitude [21]. For higher viscosity however the driving voltage has no significant effect on the volume of the head drop [137]. The head drop drags along a thinner cylindrically shaped volume, referred to as tail, that is initially connected to the fluid in the nozzle. After this tail pinches off, gradually a second spherical cap is formed at the end of the tail, thanks to capillarity. This tail droplet is accelerated by the capillary force of the tail and increases in volume while it absorbs part of the volume of the tail. The tail droplet has a velocity which is set by [12]: $u_{\text {taildrop }}=\sqrt{\sigma / \rho R_{0}}+u_{0}$, with $u_{0}$ the velocity of the tail close to the tail drop. This equation holds only for high viscosity non-elastic inks and non-stretching tails. The overall evolution of the droplet is dependent on the difference between the head drop and the tail drop velocities: If the head drop velocity is larger than the tail drop velocity, the tail will be stretched and eventually will break up in small droplets, see Fig. 6.1(a). If the head drop velocity is smaller than the tail drop velocity, the droplets will eventually coalesce into a single droplet, as in Fig. 6.1(b). For the latter case, the tail may still break up, if the typical growing time of the Rayleigh Plateau instability $[19,138]$ in the tail is smaller than the typical time that is required for the two droplets to coalesce, see Fig. 6.1(c). Breakup of the drops, which results in multiple smaller drops, is undesired in the process of inkjet printing.

Droplets moving through air experience a drag force, which depends on the Reynolds number $R e=\frac{v D \rho}{\mu}$ ) [139]. For typical macroscopic droplets with $\operatorname{Re} \geq$ $\mathrm{O}\left(10^{3}\right)$ the inertial forces dominates, and for freely falling microdrops at terminal velocity corresponding to $\mathrm{Re} \leq \mathrm{O}\left(10^{-3}\right)$ the Stokes drag force dominates. Microdrops have a small terminal velocity, typical $10 \mathrm{~mm} / \mathrm{s}$, at which the drops become increasingly sensitive to air flows, potentially deflecting the drops from its intended path. Therefore a minimal jetting velocity is required to have a reliable flight path. 


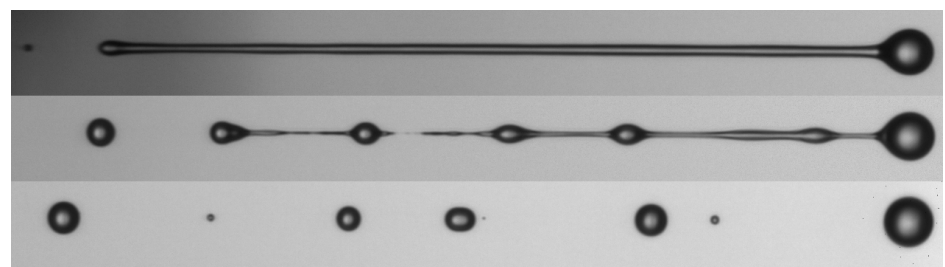

(a)

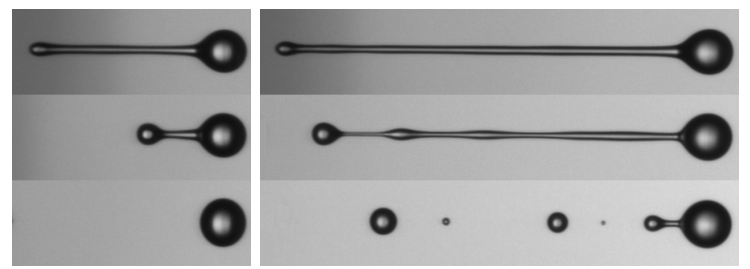

(b)

(c)

Figure 6.1: Different drop formation scenarios for different actuation amplitudes. (a) If the tail drop velocity is lower than head drop velocity, the tail will stretch and break up into smaller droplets. (b) If the tail velocity is much smaller than the head velocity, the elongated drop will contract into a single spherical drop. (c) The tail drop velocity in this case is a bit smaller than the head drop velocity. Therefore there is enough time for the tail to break up, before contraction of the head and tail drop.

Jetted microdrops with $\mathrm{Re}=\mathrm{O}\left(10^{1}\right)$ are positioned in-between the Stokes and inertiadominated regimes [140].

At higher DOD frequencies, at which droplet trains are formed, the air in-between the drops may move along with the drops, which reduces the air drag on the individual drops in the droplet train [141]. So the size of the drops, their velocity, and the DOD frequency have all an influence on the effective air drag felt by the individual drops. Viscous drag of the air on the tail however was found to be negligible for filament radius $R>\mu_{\text {air }} \mu / \sigma \rho[142,143]$, with $\mu_{\text {air }}$ the viscosity in the air and the other variables are evaluated in the ink. For the parameters of a typical hotmelt ink [14], the critical radius is smaller than $10 \mathrm{~nm}$.

Drop formation models can help our understanding of the drop formation dynamics after ejection and on the appearance of satellite drops. More importantly, numerical models allow for a much larger parameter range to be studied. It allows to scan drop optimum formation regimes, with minimal satellite drop formation at acceptable final drop velocities.

We categorize some popular CFD codes used for inkjet drop formation, these are: proprietary software CFD-ACE+ [144], FLOW-3D [18] and ANSYS FLUENT [145], open source methods such as Gerris [25, 146] or OpenFOAM [147] and solving simplifications of the NS-equations e.g. a potential flow with boundary integral calcu- 
lations (BI) [148] or the slender jet approximations [11, 12]. In order to compare the drop formation predictions of these numerical methods, it is common practice to set up benchmark cases which are calculated with the different codes. This can be done by producing a realistic drop profile with an acoustic/ interface and to calculate the drop formation process. The downside of this approach is that the numerical predictions are only compared with respect to one another, without reference to the actual situation. Alternatively the pressure input for drop formation models is chosen to match a experimental drop formation at later stages. In order to achieve this, the initial actuation pressure is refined iterative to match experimental drop formation as well as possible. T. Glatzel et. al. [149] used an experimentally measured pressure signal as initial condition and compared the pinch-off time from the nozzle and the resulting drop shape. For this case they compared the VOF codes CFD-ACE+, CFX and Fluent. They find that the CFD-ACE results match their experimental data the closest. With Fluent they could not find a pinching drop at all, and within FLOW3D the problem could not be implemented satisfactory. The pressure pulse in that work was increased by $5-10 \%$ as compared to the measured one to obtain best agreement for the drop formation.

In this work we use a novel method and start with the drop geometry and velocity, extracted from experimental recordings as the initial condition in the models. We then compare the results of the simulations to experimental data at subsequent time steps. For the experimental recordings a high speed-recording setup is used. We accurately measure the drop geometry and velocity profile at a certain instance in time and we evaluate the influence of drag on the flight of the drops. Two benchmark cases are studied: a contracting drop and a drop which breaks up into smaller satellite droplets. We compare the moment of contraction, the velocity within the drops and their volume distributions. We also compare the numerical efforts associated with each model, such as grid resolution and calculation times.

\subsection{Benchmarks cases}

In this section we describe the two experimental benchmark cases, namely a contracting tail and head drop case and a tail breakup case.

\subsubsection{Experimental method}

The time resolved axisymmetric droplet shape and velocity profiles are calculated from inkjet droplets in flight using a high speed visualization setup, as explained in Chapter 5. The one-dimensional velocity throughout the droplets at each time step are determined using a double frame recording with an interframe delay of $300 \mathrm{~ns}$, of a single droplet. Therefore small drop to drop variations of the jetting process 
will not have any influence on the determined velocity profile. The images in this chapter were recorded with a sCMOS camera (LaVision Inc., Imager sCMOS) and through a microscope (Olympus, BX-30MF) equipped with a $20 \times$ microscope objective (Olympus, SLMPLN20x). The used laser (Evergreen, Quantel) produced the illumination flashes with an interframe delay accuracy of $3 \mathrm{~ns}$. We further improved the boundary detection method and resulting accuracy by including the weighed volume of a pool of more than 10 million drops, see appendix 6.A. The experiments were performed with a prototype printhead (Océ Technologies BV.)with a nozzle diameter of $27 \mu \mathrm{m}$. A silicon oil was used as ink, since its fluid properties are more robust against external influences, e.g. absorbed dust particles, temperature and pressure variations. Also the oil does not evaporate, keeping the total droplet volume constant. The silicon oil AK10 (Wacker Chemie AG) was selected, with a viscosity of $9.3 \mathrm{mPas}$ and a density of $930 \mathrm{~kg} / \mathrm{m}^{3}$, resembling the fluid properties of the hot melt ink typically used in these printheads. The surface tension is $20.2 \mathrm{mN} / \mathrm{m} ; 1.5$ times smaller than that of a typical hot-melt ink.

\subsubsection{Benchmark case 1: contracting tail and head drop}

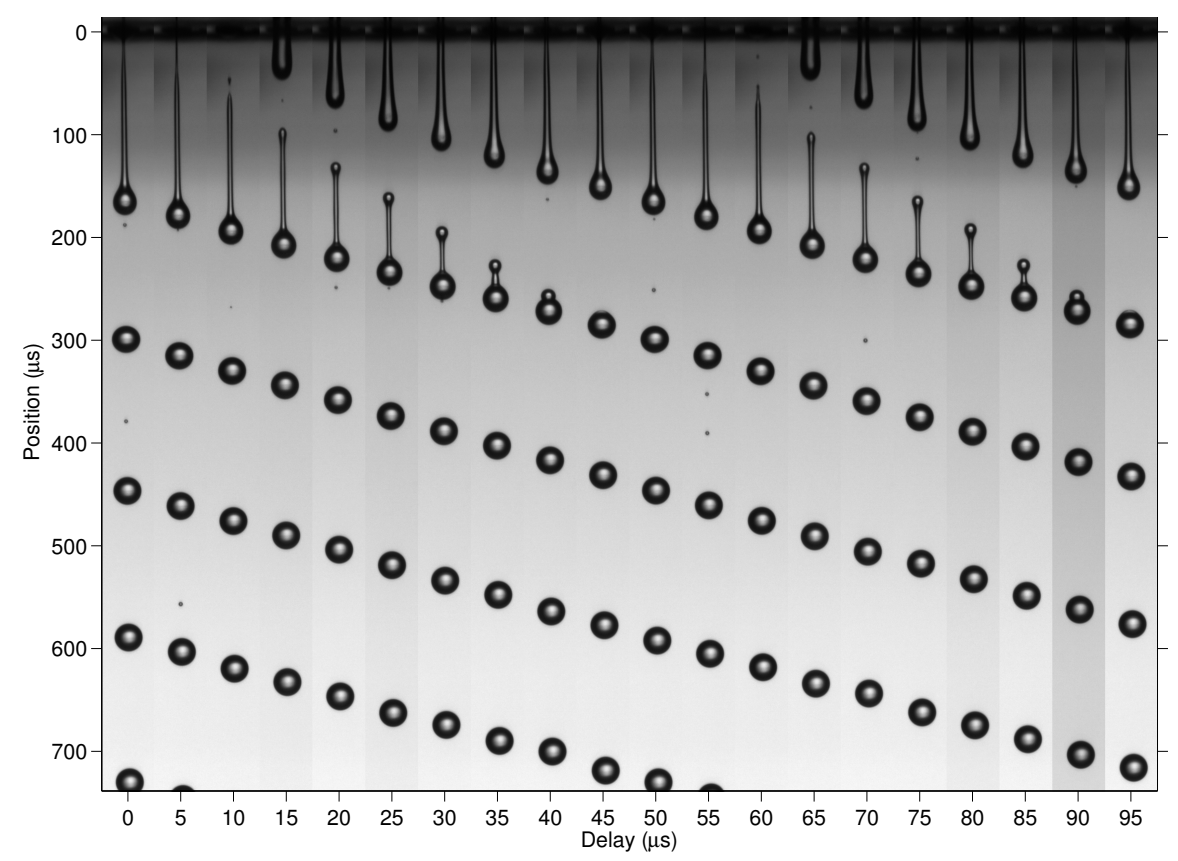

Figure 6.2: Benchmark case 1: Single contracting drop case actuated at $20 \mathrm{kHz}$. At the top of the figure the nozzle plate is visible, and the drops are jetted downwards. The nozzle is at position $0 \mu \mathrm{m}$. Shortly after jetting, the drops are elongated and contract then to a spherical drop. 
In this case a droplet appears with a head and tail drop which contract over time. This can be considered to be the ideal jetting situation; the droplets nicely contract to become a single spherical drop. Consequently, the velocity of the head drop must be lower than the typical tail drop velocity. The droplets were actuated at a frequency of of $20 \mathrm{kHz}$, creating a drop stream with some co-flow of air. This case allows for a comparison of the time of the contraction, the velocities of the head and trail drop and the total volume/momentum conservation.

The drop formation for the first drop case was recorded with delay steps of $100 \mathrm{~ns}$. Frames of the recording with subsequent delay steps of $5 \mu \mathrm{s}$ are shown in Fig. 6.2, showing every $50^{\text {th }}$ recording. The fact that every frame a different drop was recorded, becomes only apparent from the small satellite drops, located at arbitrary locations. The velocity of the head drop is much smaller than the tail drop velocity, and the drops coalesce approximately $35 \mu$ s after the drops pinch off from the nozzle. The average volume per drop, $9.63 \mathrm{pL} \pm 0.02 \mathrm{pL}$, was found by weighing $11.7 \times 10^{6}$ drops.

We first discuss the analyzed geometry and velocity of the recorded drops. The radius and velocity analysis results are plotted in Fig. 6.3. The values of the radius and velocity profiles along the drops length are represented by the colors and are plotted at the drops positions and for the time after the actuation. The drops move downwards in time and at $t=0 \mu$ s the drop series indicated with arrows was actuated. This is also the drop series that is used in the further comparison. Several microseconds before the pinch-off, a secondary tail of sub-micron scale emerges. At this scale the fluid boundary cannot be imaged accurately, giving rise to variations in the evaluated droplet.

To verify the velocity from the double frame recording in the head and tail drop, we compare it to velocity obtained from the average motion of different drops. To obtain this time averaged velocity, we first determine the location of the head and tail drops from the maximal radius inside the head and tail drops. Then we determine piecewise linear fits of the positions of 50 different drops (over $5 \mu \mathrm{s}$ ). Taking the derivative of these linear fits results in the average velocity of 50 different drops. The time averaged velocities are plotted at the center of the evaluated timespans in Fig. 6.4, along with the velocities obtained from the double frame analysis. The velocity of the head drops decreases from $3.0 \mathrm{~m} / \mathrm{s}$ to $2.5 \mathrm{~m} / \mathrm{s}$ during contraction where the tail velocity increases form $6.0 \mathrm{~m} / \mathrm{s}$ to $6.5 \mathrm{~m} / \mathrm{s}$. After contraction, the velocity of the drop at $120 \mu \mathrm{s}$ after actuation was found to be $3.0 \mathrm{~m} / \mathrm{s}$. The deceleration of the tail drop before the contraction and its acceleration due to the coalescence of the head and tail drop are found to very well match the mean velocity of the droplets. 

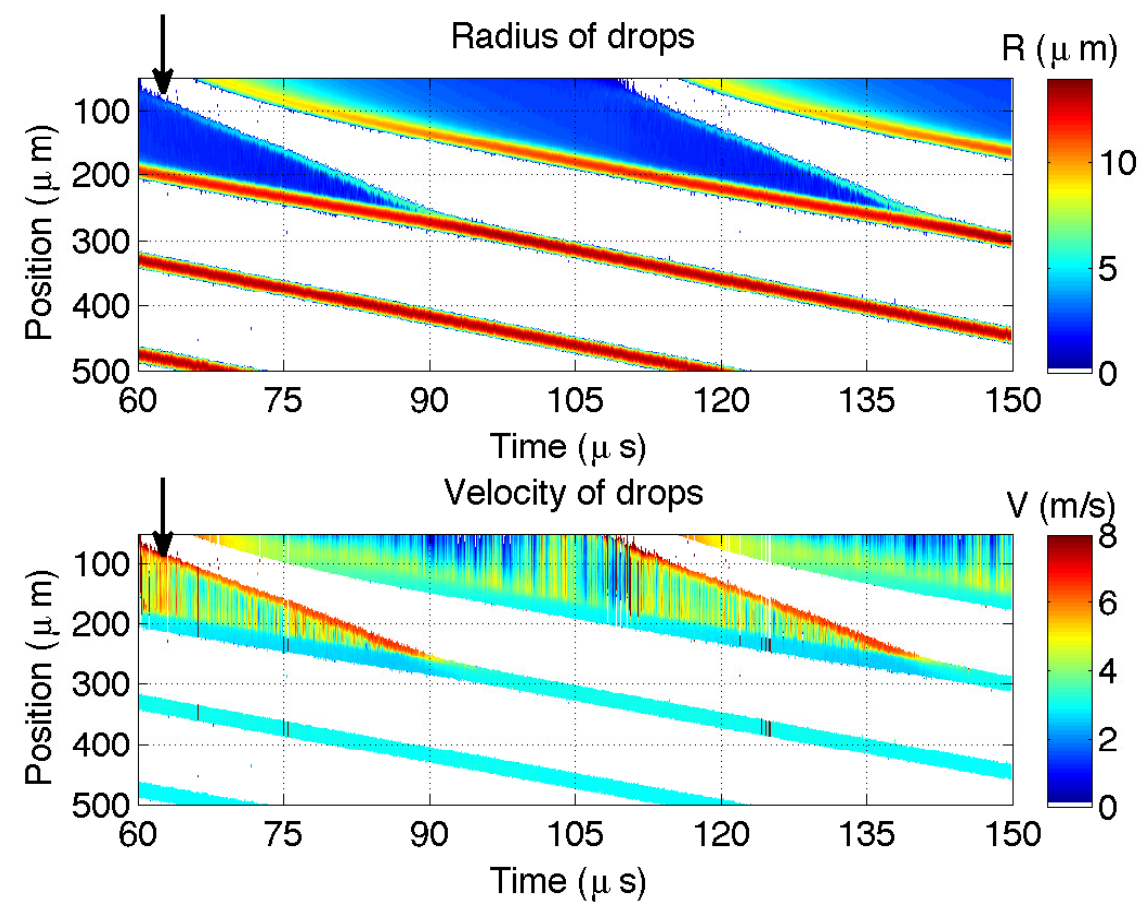

Figure 6.3: Droplet analysis benchmark 1. At $t=0$, the droplet indicated with arrows is actuated. The nozzle plate is at position $0 \mu \mathrm{m}$, the drops were measured at $46 \mu \mathrm{m}$ from the nozzle. The tail drop velocity is about $6.5 \mathrm{~m} / \mathrm{s}$ and the head velocity is found to be minimal $2.5 \mathrm{~m} / \mathrm{s}$. 


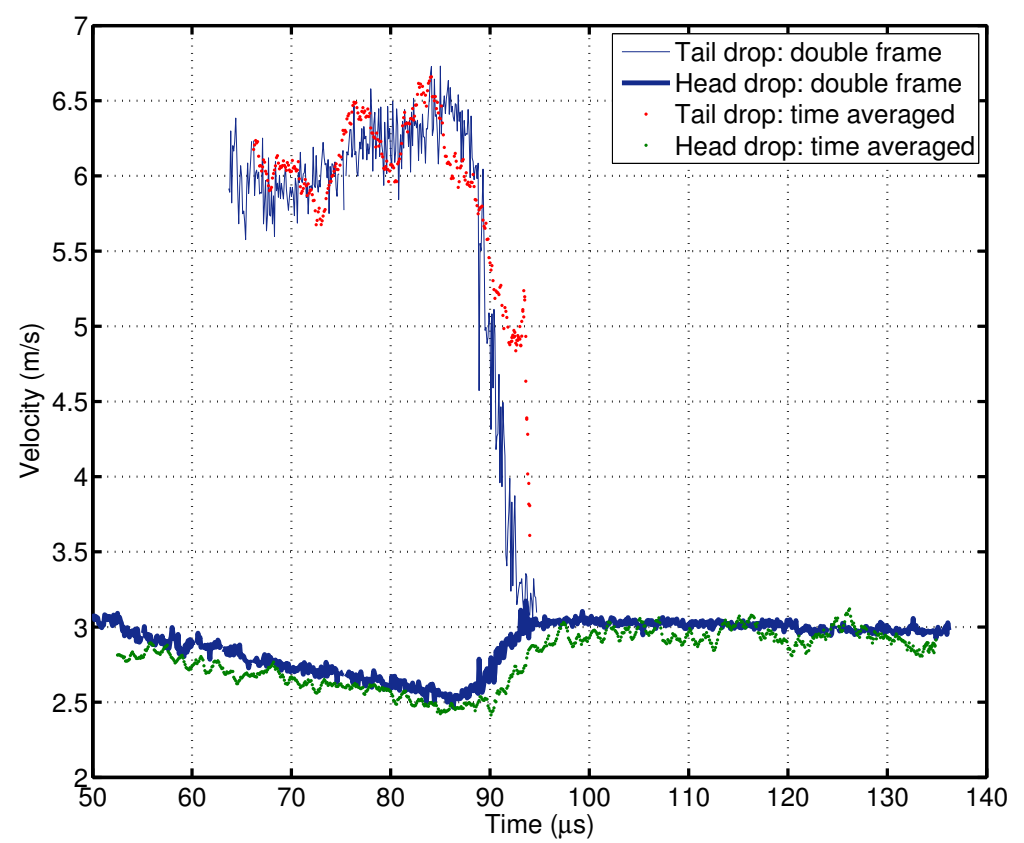

Figure 6.4: Experimental velocity analysis verification for benchmark case 1 . The velocities of the head (thick line) and tail drop (thin line) calculated with the double frame recording of single drops, is compared to the time averaged velocity over 50 different drop (red/green dots). 


\subsubsection{Benchmark case 2: Tail breakup}

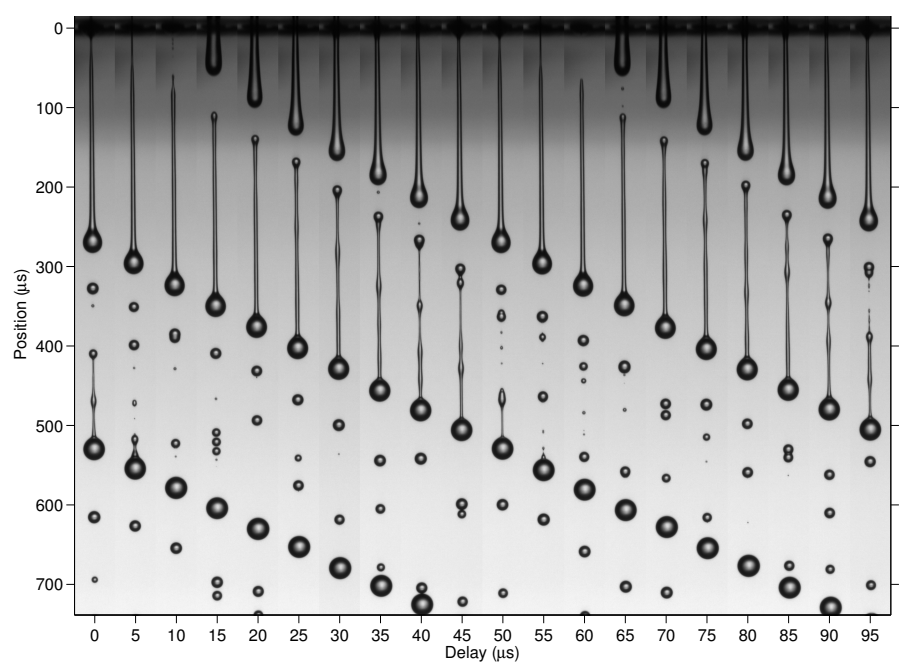

Figure 6.5: Benchmark case 2: For this stronger actuation, the droplet breaks up in small droplets. The recorded frames are indicated with an increasing time delay with $5 \mu$ s time steps. The droplet breakup is a stochastic process which results in a different droplet distribution for every actuation.

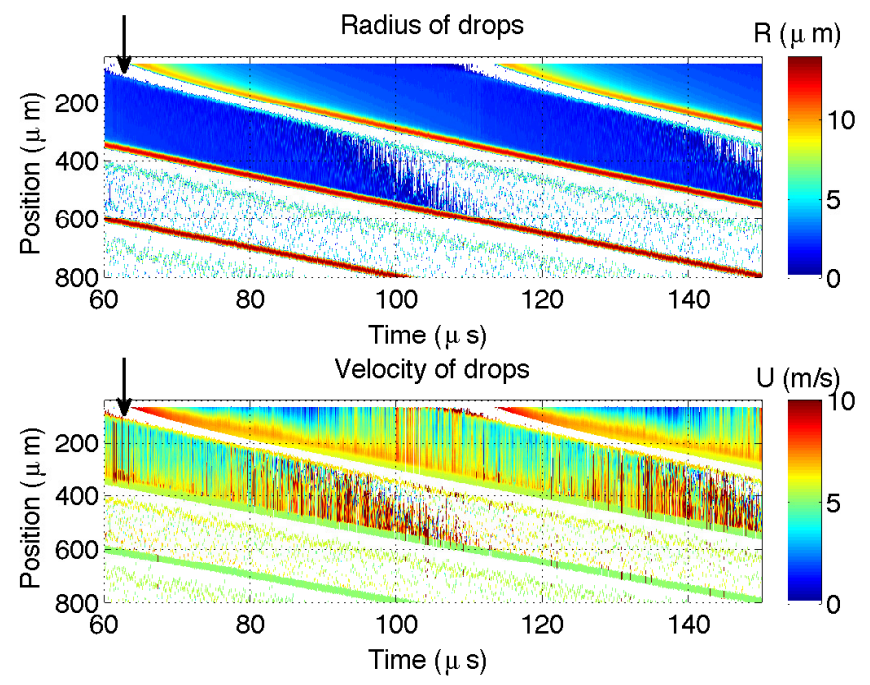

Figure 6.6: Droplet analysis of benchmark case 2. At $t=0 \mu \mathrm{s}$, the droplet is actuated (indicated with an arrow). The tail drop velocity is $6 \mathrm{~m} / \mathrm{s}$ and the head velocity is found to be close to $5.2 \mathrm{~m} / \mathrm{s}$. 


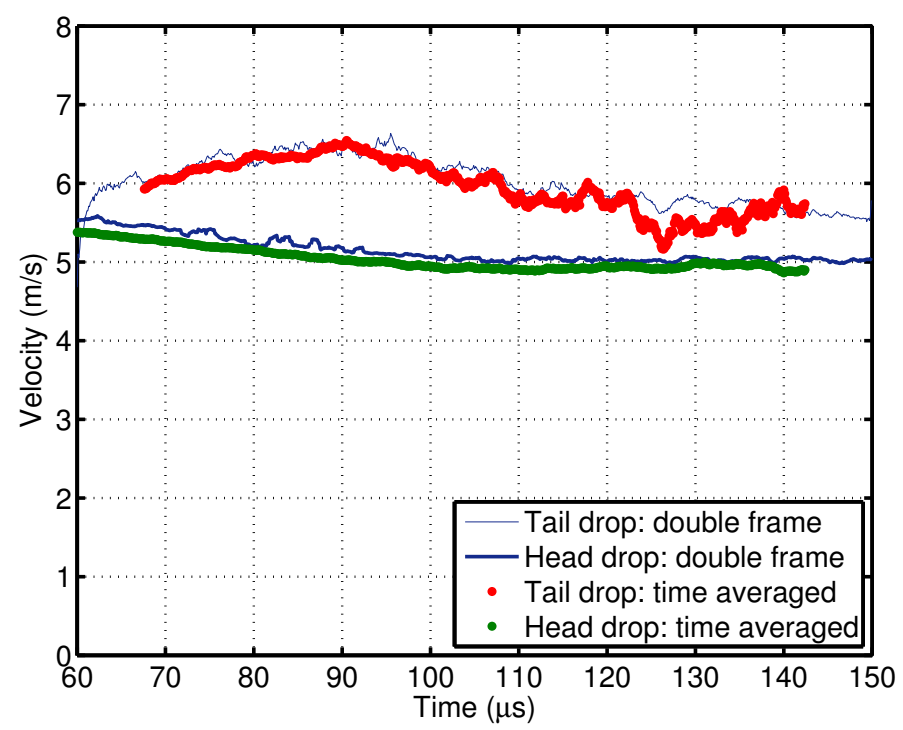

Figure 6.7: Comparison of velocity analysis of experiments between the double frame method of a single drops and the time averaged method of different droplets. The calculated double frame velocities of the head (thick line) and tail drop (thin line) were averaged over $2 \mu \mathrm{s}$. The time averaged velocity (red/green dots) were determined over 150 different droplets $(15 \mu \mathrm{s})$.

In the second benchmark case, a stronger actuation was used, resulting in a head drop velocity only slightly smaller than the tail drop velocity. Therefore the head and tail drops stay separated long enough for the tail to break up. Using a head velocity which is lower than the tail velocity ensures that the drops stay separated from drops of the previous actuations. The drops in this benchmark were also jetted at $20 \mathrm{kHz}$ and recorded with time steps of $100 \mathrm{~ns}$. Fig. 6.5 shows snapshots of the drops, with increasing timestep delays of $5 \mu \mathrm{s}$. The filament between head and tail drop breaks up due to the Rayleigh-Plateau instability, namely in a larger head drop and a series of smaller drops. The initial perturbations of the tail cause the drop to break up differently for each actuation, which is reflected in the drop to drop variations in the measurements. The total mean drop volume per actuation, determined by weighing $11.7 \times 10^{6}$ drops, was $13.16 \mathrm{pL} \pm 0.02 \mathrm{pL}$.

The radius and velocity data were extracted from the recordings and are plotted in Fig. 6.6. At $t=0 \mu \mathrm{s}$, the printhead was actuated, to produce the drop indicated with an arrow. During the breakup process (between $t=90$ and $110 \mu \mathrm{s}$ ) the tail radius occasionally became smaller than $1 \mu \mathrm{m}$, which was too thin to image quantitatively and led to a larger inaccuracy of the velocities in the tail. 
The velocities obtained from the double frame analysis (averaged over $2 \mu$ s) were verified against the time averaged velocity of the head and tail drop over $15 \mu$ s, see Fig. 6.7. The strong drop to drop variation in this case demanded longer averaging windows than in benchmark case 1 . The velocity profiles from the dual shut recordings agreed with the global velocity of different drops. We found a typical head drop velocity of $5.2 \mathrm{~m} / \mathrm{s}$ and a tail drop velocity of $6.0 \mathrm{~m} / \mathrm{s}$.

The calculated volumes are plotted in Fig. 6.8. The drops break up in head drops of typical $10.5 \mathrm{pL}$ and tail drops with an average volume of $1.5 \mathrm{pL}$. The filament typically breaks up into a series of satellite drops of $1 \mathrm{pL}$ and even smaller drops with a volume $<0.2 \mathrm{pL}$. The sum of the volumes of the drops is found to match the volume determined from the weighing experiment, which is indicated in Fig. 6.8a with the dashed line. In $6.8 \mathrm{~b}$ the PDF of the droplet volume is shown. It indicates how the volume of a drop is distributed after breakup. The PDF of all experiments is calculated by adding up the volume of drops within each volume range box and dividing by the total volume of all drops and by the width of the evaluated volume box. The integral over the PDF results in unity, $\int_{0}^{\infty} P D F(V) d V=1$.

\subsubsection{Drag}

From the experiments, we only obtain the flow in the fluid of the drop and not in the surrounding air. For a better comparison with simulations, we investigated the influence of drag on the drop formation precess, see Appendix 6.B. The result was that in out experiments the velocity reduction induced by air drag is less than $7 \%$, in a $100 \mu$ s time window which was typical in our experiment. To find out to what degree the co-flow of the air between the droplets reduces the air drag on the individual drop, we compares the drag induced velocity reduction of our droplet train experiments to that of a single drop flying through air. We find that the co-flow of the air reduced the air drag by $17 \%$ for $10 \mathrm{kHz}$ and $32 \%$ for $20 \mathrm{kHz}$, as than the drops are packed more closely together. From this we conclude that the drag on the individual drops in our experiments is approximated better by a single drop moving through quiescent air than by a flying drop without any air drag. 


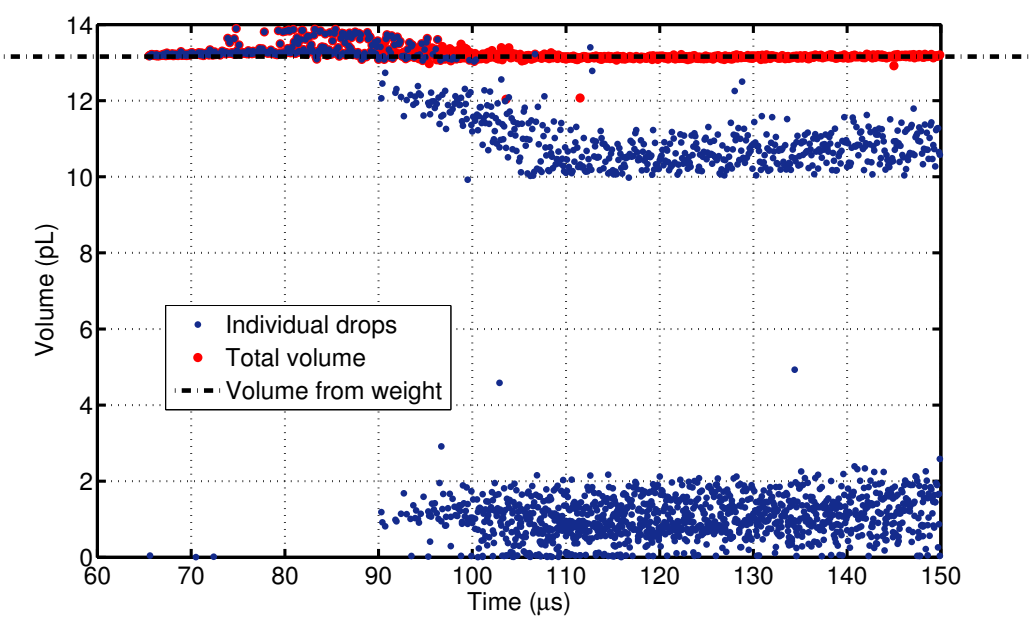

(a)

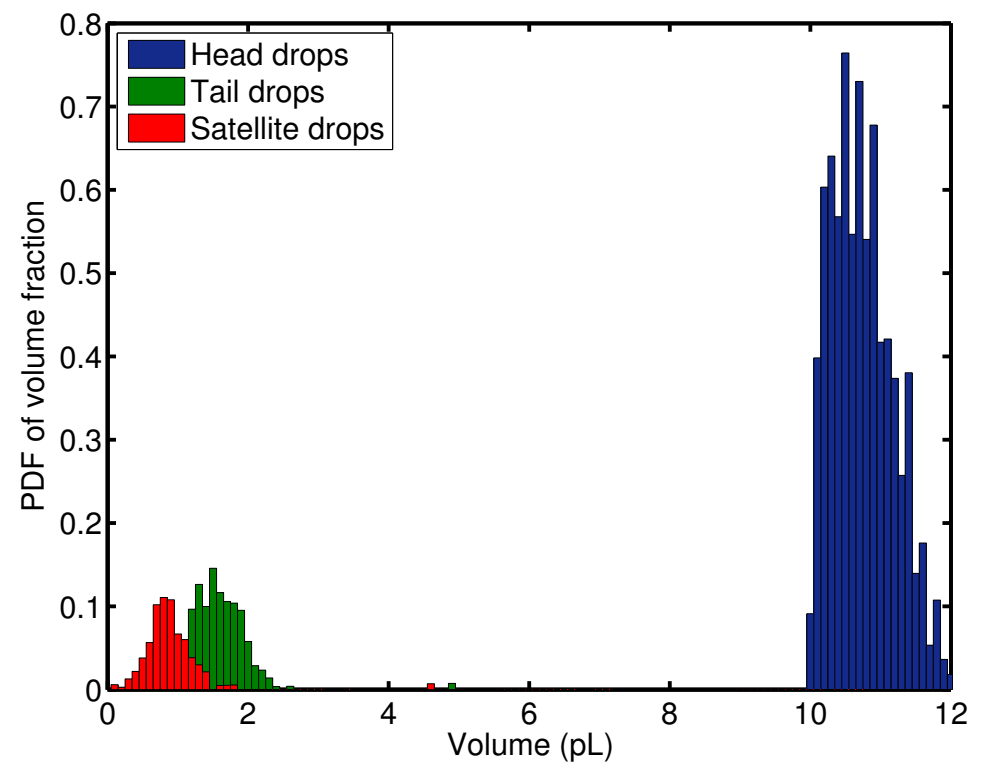

(b)

Figure 6.8: (a) Volume of individual droplets (blue) and their total volume of drops (red). (b) Probability density function (PDF) of droplet volume after droplet breakup. The volume is scaled by the total volume of all drops, and the width of the volume discretization boxes. The drops typically break up into a large drop $(10.5 \mathrm{pL})$, tail drops (1.5 pL), and satellite drops (1.0 pL). 


\subsection{Numerical cases}

We now come to the three numerical methods that we used to simulate above described droplet formation cases 1 and 2. In principle to calculate the droplet formation process, the full Navier-Stokes equations have to be solved, together with solving the free surface of the liquid gas interface. In this section we give an overview of the numerical methods and codes used in this work. The numerical codes, fluids in the simulation and fluids in the simulation are summarized in Table 6.1.

\subsubsection{Fluent}

Fluent [145] is a commercial Volume of Fluid (VOF) [150] type code, which is an Navier-Stokes solver with an advection scheme for an additional variable next to the velocities. This variable specifies the fraction of fluids within the grid cells. From this volume fraction function the fluid boundary can be estimated. VOF excels in the minimum amount of information that is stored and the good conservation of volume. A downside of the technique is the inferior accuracy of the curvature of the free interface [25]. In the droplet formation process at the microscale surface tension plays an important role, and therefore does require an accurate determination of the curvature of the surface. VOF codes use several techniques to improve the accuracy of the reconstruction of the interface. To improve the description of the surface in Fluent node based smoothening ( $\mathrm{NbS}$ ) was used, which smoothes the VOF field using the values at the nodes instead of the values in the cells. In the Fluent simulations ${ }^{\dagger}$ both the flow in the liquid in the drop and in the surrounding gas were calculated. The system was solved assuming axisymmetric symmetry. A fixed time step and a fixed mesh size were used, with settings optimized for jetting of picoliter-sized drops.

${ }^{\dagger}$ The Fluent simulations in this work have been performed by W. Wagenaar, Infinite Simulation Systems B.V.

\begin{tabular}{|c|c|c|c|}
\hline Code & Method & Differencing method & Fluids \\
\hline \hline Fluent & VOF + NbS & Finite volume & Immiscible/ \\
& & & multiphase \\
Gerris & VOF + HF & Finite volume & Multiphase \\
Slender jet & RK 23 solver & Finite difference & Single fluid \\
\hline
\end{tabular}

Table 6.1: Summation of used numerical models and the applied methods. Volume of Fluid (VOF) codes are extended with Node based Smoothening ( $\mathrm{NbS}$ ) and Height Functions (HF). For the slender jet a Runga-Kutta (RK) solver is used. 


\subsubsection{Gerris}

Gerris [25] is a VOF code that uses an height function (HF) [151] calculated from the VOF volume fractions for this purpose and is used to calculate the local curvature of the surface. Gerris simulations include both the liquid in the drop and the surrounding gas. In the present case the system was solved on an axi-symmetric grid. The mesh and time steps were automatically refined depending on the simulation conditions. For the Gerris simulations $s^{\ddagger}$ the reference frame was chosen to move with the centre of mass of the drop. Such calculations were much cheaper than those for drops moving through the spatial domain, due to the size reduction of this calculation domain. The initial air velocity was chosen to be zero around the drop. This implied that in the lab frame there is an air flow along with the drop with the velocity of the center of mass of the drop.

\subsubsection{Slender jet approximation}

The NS equations for droplet formation can be simplified by means of the slender jet approximation [11]. The simplification is based on the fact that the aspect ratio of radial to longitudinal scale of the flow is small. Assuming axial symmetry and applying the slender jet approximation to the NS-equations results in a one-dimensional set of partial differential equations (PDE), which connect the radius, velocity, and pressure along the droplet. Regularization of the surface tension is required to prevent singularities that occur at pinch-off and coalescence of drops [12]. Using finite differencing, the PDE's were locally approximated by ordinary differential equations (ODE), which were solved with a time-adaptive Runga-Kutta (Bogacki-Shampine) method. The slender jet model calculated only the liquid of the drops, neglecting the surrounding gas.

\subsection{Comparison}

The models generally use a finer grid than the experimental spatial resolution of $0.33 \mu \mathrm{m}$ and therefore the initial radius has to be linearly interpolated.

\subsubsection{Benchmark 1}

We run the simulations which were started after the droplet pinched off from the nozzle, at $63.7 \mu$ s after the actuation. At this time step the volume and momentum was found to be $9.64 \mathrm{pL}$ and $2.84 \times 10^{-11} \mathrm{~N} \mathrm{~s}$, respectively. The grid and temporal resolutions of the different simulations and calculation times are indicated in table 6.2. The

\footnotetext{
†The Gerris simulations in this work have been performed by S. Wildeman, POF group, University of Twente.
} 


\begin{tabular}{|c|r|r|r|r|}
\hline Model & $\begin{array}{r}\text { Mesh resolution } \\
(\mathrm{nm})\end{array}$ & $\begin{array}{r}\text { Mesh size } \\
(\text { cells })\end{array}$ & $\begin{array}{r}\text { Time } \\
\text { resolution }(\mathrm{ns})\end{array}$ & $\begin{array}{r}\text { Calculation time } \\
\text { (hours) }\end{array}$ \\
\hline \hline Fluent & 250 & $5.9 \times 10^{5}$ & 10 & $127(4$ cores $)$ \\
Gerris & var $19-2500$ & var $1 \times 10^{4}$ & var 3.9 & $5.0(1$ core $)$ \\
Slender jet & 100 & $7.7 \times 10^{3}$ & var 0.065 & $1.0(1$ core $)$ \\
\hline
\end{tabular}

Table 6.2: Benchmark 1: Simulation resolution and calculation times (elapsed real time). For the variable parameters, which are determined in the simulation, typical or average values are indicated. In Gerris a smaller domain is used, with adaptive meshing, and therefore less mesh cells are needed in the simulation. The slender jet approximation only has cells in one dimension, resulting in less total cells.

\begin{tabular}{|c|c|c|c|}
\hline & $\begin{array}{c}\text { Maximal velocity } \\
\text { tail drop }(\mathrm{m} / \mathrm{s})\end{array}$ & $\begin{array}{c}\text { Moment of } \\
\text { contraction }(\mu \mathrm{s})\end{array}$ & $\begin{array}{c}\text { Final drop velocity } \\
(\mathrm{m} / \mathrm{s})\end{array}$ \\
\hline \hline Experiment & $6.5 \pm 0.3$ & $93.6 \pm 1$ & $3.04 \pm 0.04$ \\
Fluent & 6.65 & 92.0 & 2.99 \\
Gerris & 6.87 & 91.7 & 3.15 \\
Slender jet & 6.84 & 91.6 & 3.17 \\
\hline
\end{tabular}

Table 6.3: Benchmark 1: Table of experimental and numerical results for comparison. The final drop velocity is evaluated $120 \mu$ s after the actuation.

slender jet model is much faster than the Fluent simulation due to the 1-dimensional and single fluid nature of the model. The Gerris code calculation time was also shorter, due to adaptive time and mesh resolution and the smaller spatial domain, resulting in a smaller mesh size.

The 1-dimensional representations of the radius and the velocity in the main direction of the drop are plotted in Fig. 6.9 and Fig. 6.10. In these figures the simulated profiles are continuous, contrary to the experiment, which was taken from a different drop for each time step. The drop evolutions calculated with the 3 models give results which resemble the experiment. To quantify the moment of contraction, the positions of the head drop and the tail drop were determined and from local linear fits the intersection time was evaluated. The results are shown in table 6.3. We find that the moments of contraction from the simulations are 1-2 $\mu$ s earlier than in the experiments. From the drop positions we also extracted the velocities in the head and tail drops. These velocities are plotted in Fig. 6.11 for the experiment and the simulations. The decrease in the velocity of the head drop during the contraction phase of all simulations agree very well with the experimental data. The tail velocity in the simulations up to $85 \mu$ s after the actuation is strikingly similar in the slender jet approximation and in the Gerris simulations, but were found to be higher than the average tail drop velocity in the experiments. The Fluent calculation gave the 


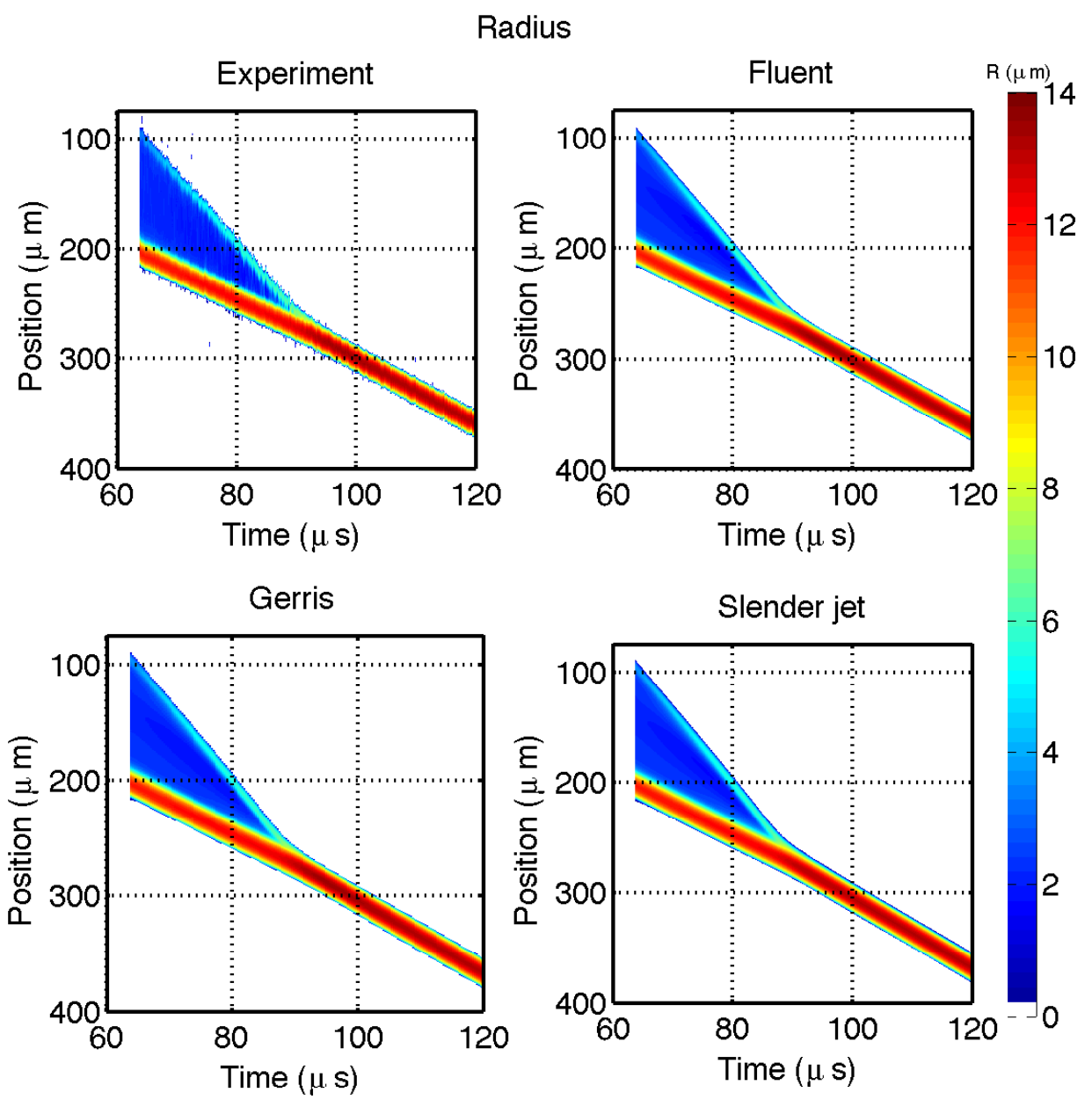

Figure 6.9: (a) Radius profiles of the experimental data and the numerical simulations. The experiments and simulations show a similar contraction behavior. These images indicate that very similar drop contractions have been calculated with the numerical models. 


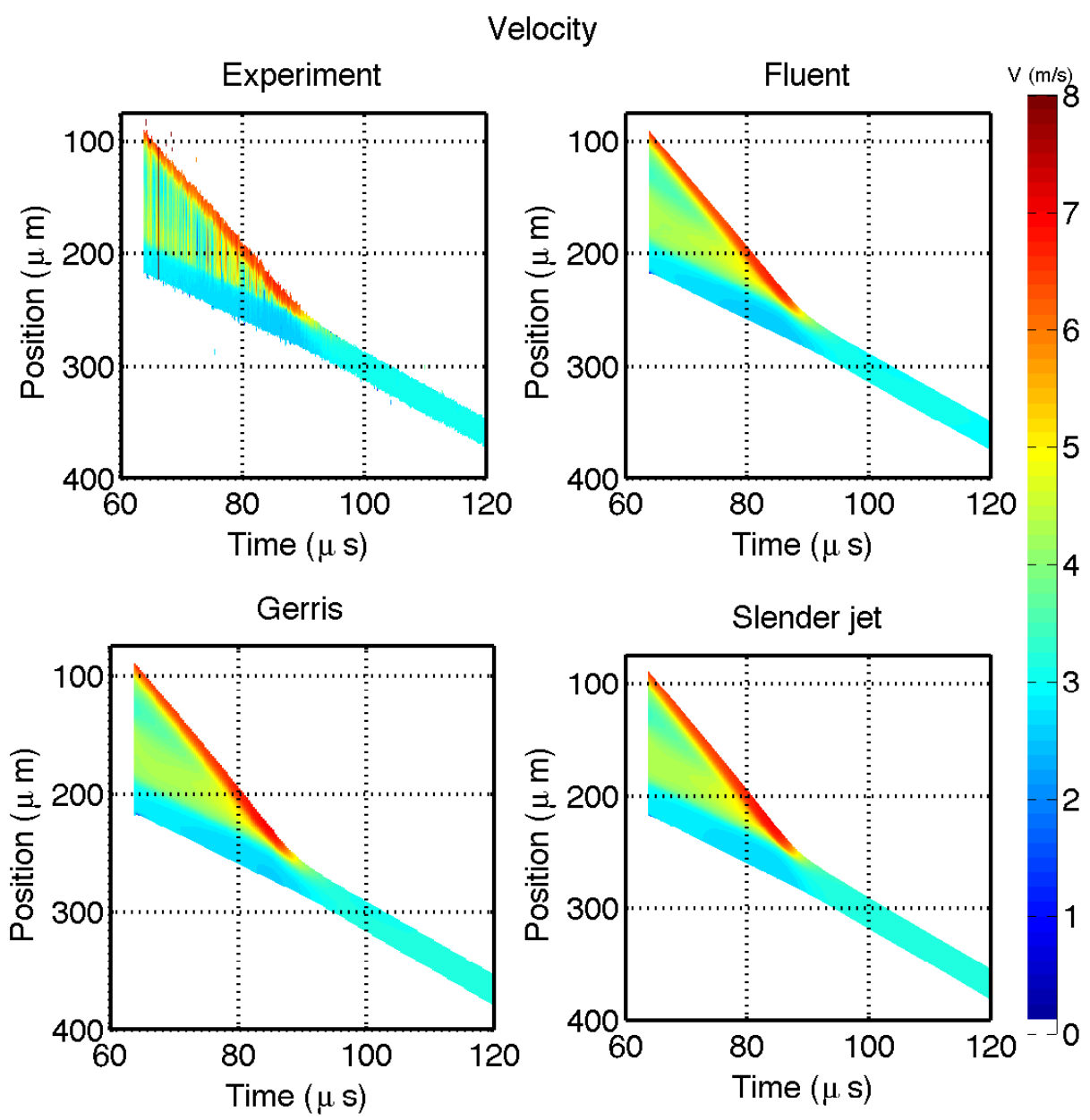

Figure 6.10: Velocity profiles of the experimental data and the numerical simulations. These images indicate that very similar drop contractions have been calculated with the numerical models.

lowest tail velocity which was closer to the average velocity in the experiments. The earlier contraction times in the simulations are caused by these larger calculated tail drop velocity. For comparison we have put the maximal velocities of the tail and the final velocity of the drop at time $120 \mu$ s in table 6.3. The final velocity for the Fluent simulation decreases with time as in experiment, due to the interaction with air. In the other simulations the velocity stay constant over time, since the slender jet code neglects the air and in Gerris there is almost no velocity difference, between the air and the drop. 


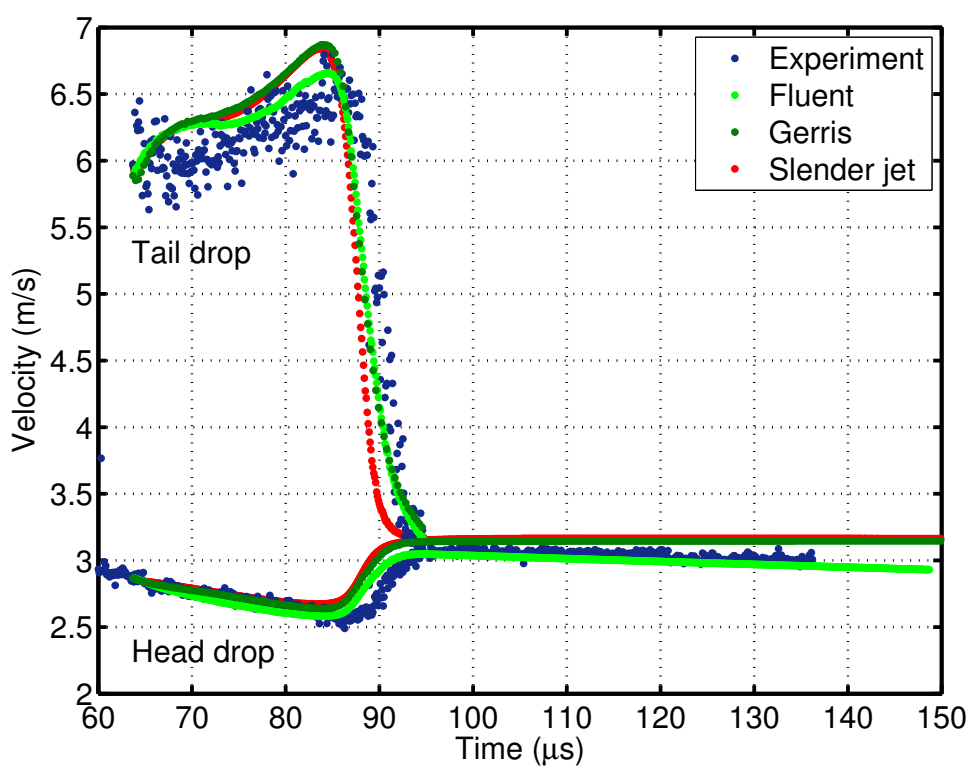

Figure 6.11: Case 1: Velocity comparison of head and tail velocity. The velocities of the head drops are equal to those of the experimental head drop. However the velocity of the tail drop is slightly larger in the numerical simulations than the general trend in the experiment, causing the drop to coalesce about $1-2 \mu$ s prior to those in the experiments.

The volume and momentum conservation of the simulations are compared to the experiment in Fig. 6.12. In the models the mass is well conserved: The mass loss is only $0.03 \%, 0.01 \%$ and $<0.01 \%$, for Gerris, slender jet approximation, and Fluent respectively. In the experiments the momentum of the drop decreases from $2.84 \times 10^{-11} \mathrm{~N}$ s to $2.67 \times 10^{-11} \pm 0.03 \mathrm{~N} \mathrm{~s}$, due to the interaction with the air. In the Fluent simulation the momentum decreases due to air drag to $2.63 \mathrm{~N} \mathrm{~m}$, i.e. slightly overestimating the drag. For the simulations of the one-dimensional slender jet model the momentum is conserved within $0.08 \%$ of the initial momentum. In the Gerris simulations with static air the momentum decreases with maximal $0.35 \%$ within the contraction phase, which is caused by the viscous dissipation in the drop and momentum transfer to the surrounding air.

\subsubsection{Benchmark 2}

Now we present the simulation results for benchmark case 2. The grid and time resolutions are depicted in table 6.4. Since the drops in this case have a higher velocity, they mover over a larger distance. 


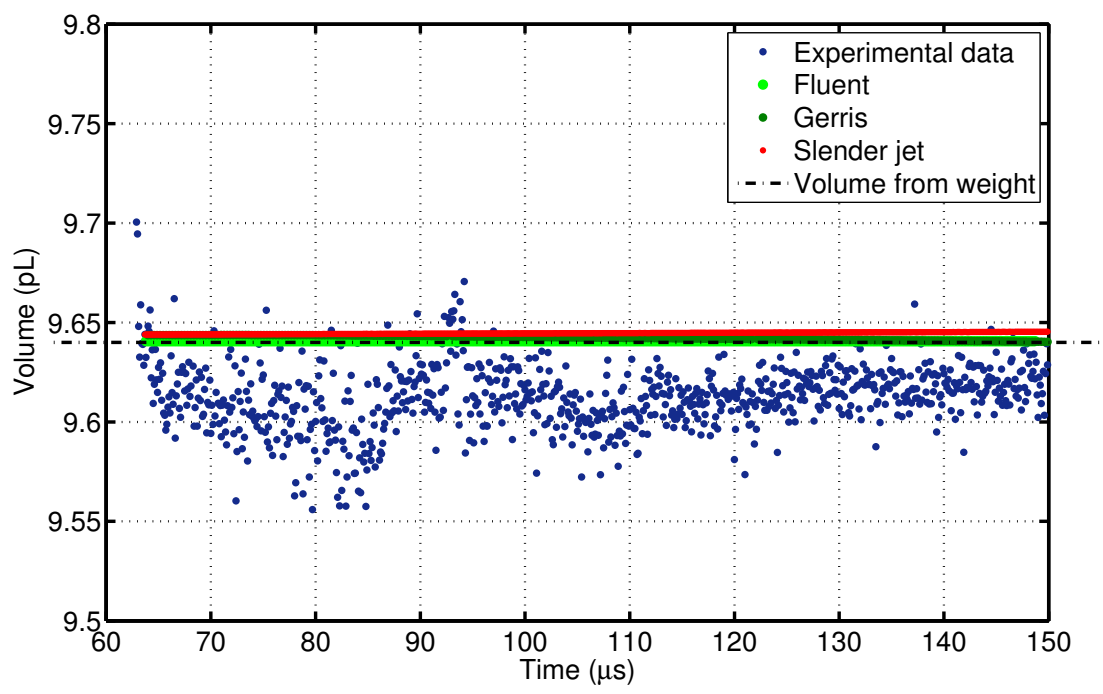

(a)

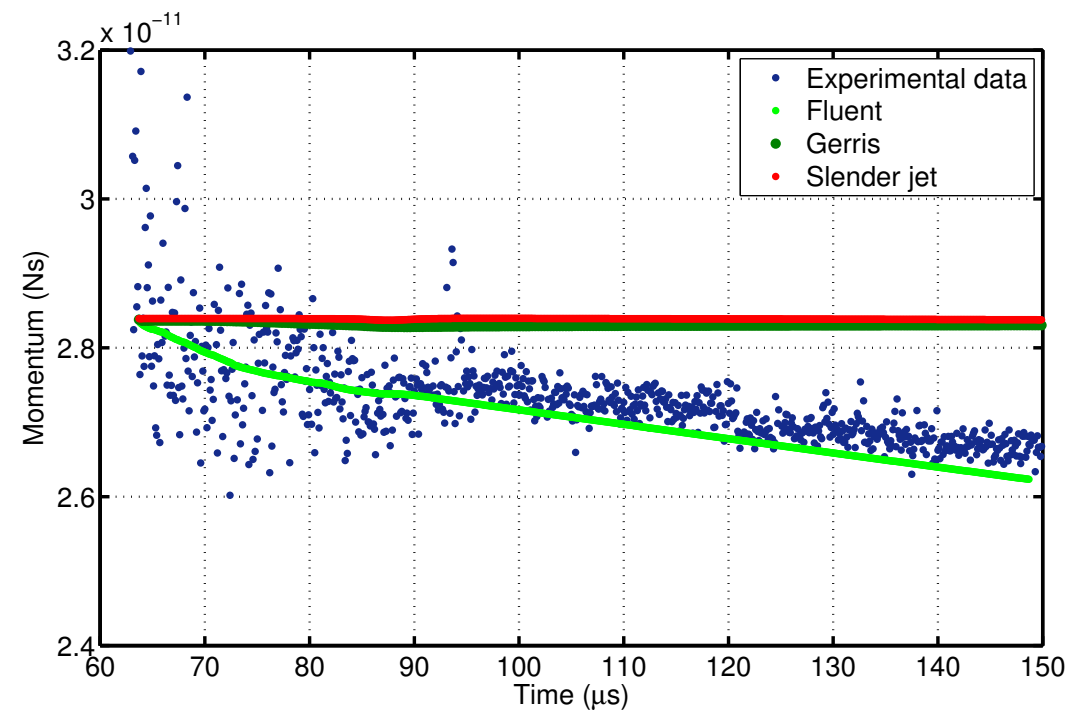

(b)

Figure 6.12: Benchmark case 1: Volume and momentum of the drops as function of time for the experimental data set and the numerical simulations. Both the volume and momentum are conserved in the models without air interaction. The experimental drop volume used as initial condition, is slightly larger than the average measured volume in the measurements. The deceleration of the drop after the contractions is slightly over estimated by Fluent, which assumes quiescent air. In the other models no air drag is included. 


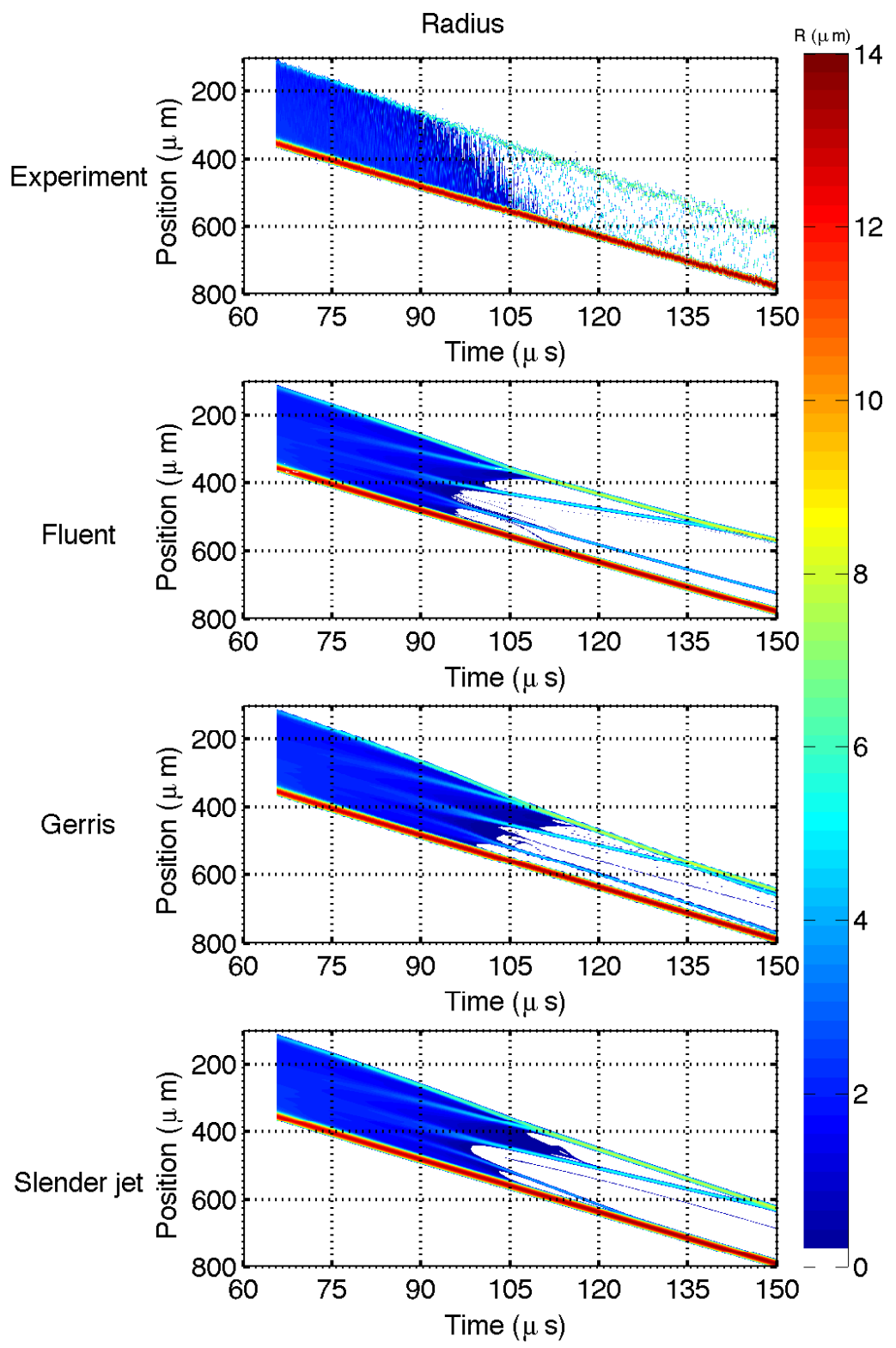

Figure 6.13: Benchmark case 2: Radius profiles of the experimental data and the numerical simulations. The experimental data shows a different drop for every time step, which reflects the stochastic breakup. The numerical models calculate similar breakup and coalescence pattern for these drops. In the Gerris simulation the drops coalesce takes much longer due to the presence of air between the drops. 

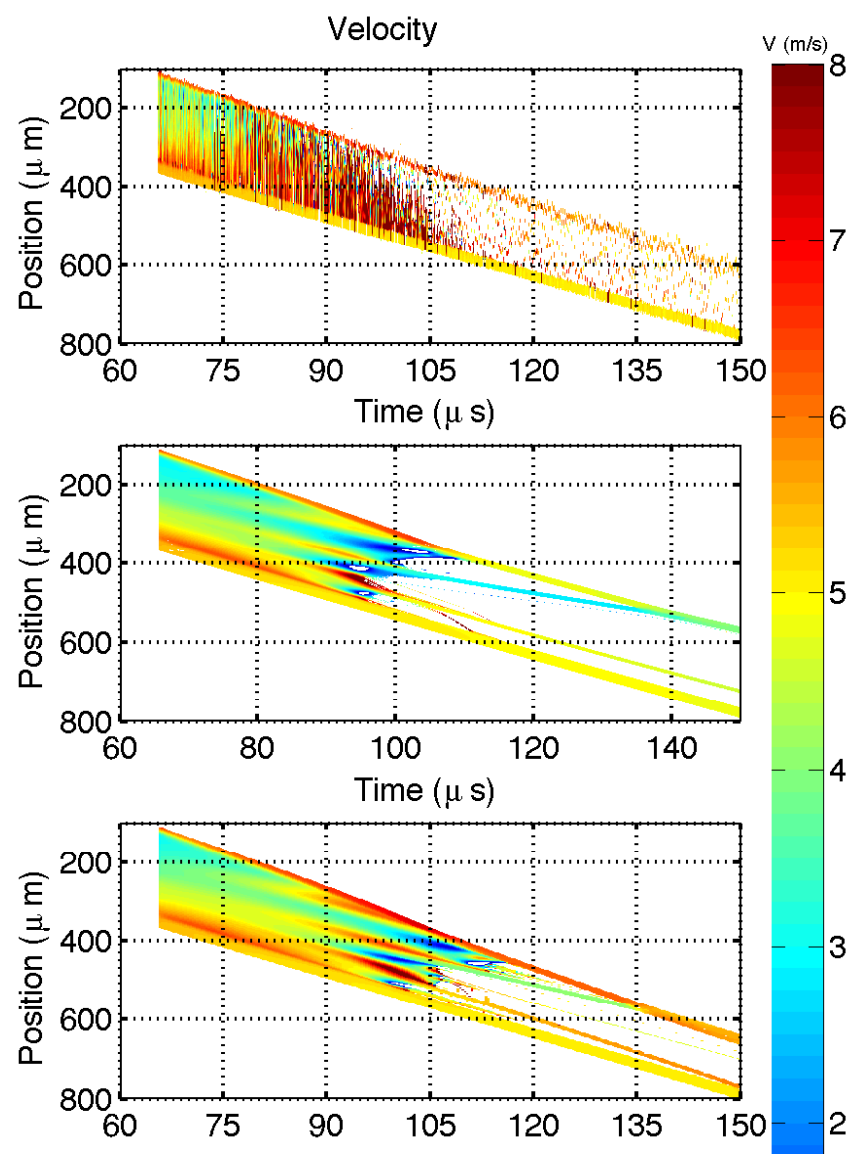

Time $(\mu s)$

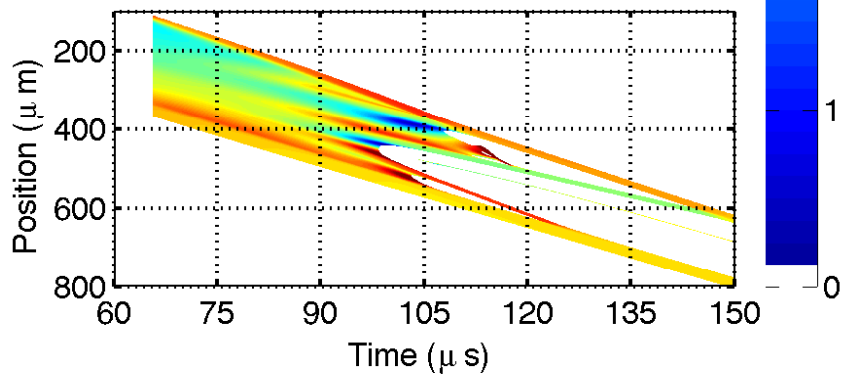

Figure 6.14: Benchmark case 2: Velocity profiles of the experimental data and numerical simulations. 
In the slender jet model, the same mesh and time span were used as for benchmark case 1, resulting in the same calculations time. The other models use a larger spatial domain, resulting in longer calculation times than for the first case. The simulated radius and velocity profiles of the drops are plotted next to those of the experiments in Fig. 6.13 and Fig. 6.14. Again, in the experimental data each recorded time step is a different droplet, but the in the simulations the drop development for the specific drop is calculated which is chosen as initial condition. All three models predict the appearance of a head and a tail drop and some smaller satellite drops as in the experiment, see in Fig. 6.13. Most satellite drops move relatively in the direction of the head or the tail drop, and coalesce with the even smaller satellite drops on their path. The velocities of these satellite drops are different from model to model. In the slender jet simulation, these drops coalesce instantly when they impact on one another. In the Gerris simulations the coalescence takes much longer, due to the presence of air in between the drops. The satellite drop in this simulation, which travels towards the tail drop, never actually coalesces with the tail drop, but is found to bounce off and fly along at the same velocity. For this particular situation a very small satellite drop $(<1 \mu \mathrm{m}$ radius was present in between these drops, which may have contributed to the non-coalescence. Also in Fluent we found the non coalescence of a very small satellite and tail drop with volumes of $7.5 \times 10^{-5} \mathrm{pL}$ and $2.8 \mathrm{pL}$, respectively.

In the experimental data we found a large number of occasions, in which the drops are close to one another, or with three in a row, see also in Fig. 6.5. Most of the time the velocity profiles show that the drops were moving towards one another, but in 5 out of 1000 recordings a satellite drop was found next to a head or tail drop, with equal velocity. This makes it plausible that retarded coalesce does take place for some cases.

We compare the volume and momentum conservation over all the drops of the simulations in Fig. 6.15. In the experiment the total momentum is found to decrease, as in Benchmark case 1. The volumes and momentum in the slender jet model were conserved in the simulations within $0.05 \%$. The momentum in the Gerris simulation was found to increase by $0.3 \%$, where the volume decreased by maximal $0.05 \%$.

The Fluent simulation shows a strong decrease in the total momentum of the

\begin{tabular}{|c|r|r|r|r|}
\hline Model & $\begin{array}{r}\text { Mesh resolution } \\
(\mathrm{nm})\end{array}$ & $\begin{array}{r}\text { Mesh size } \\
(\text { cells })\end{array}$ & $\begin{array}{r}\text { Time } \\
\text { resolution }(\mathrm{ns})\end{array}$ & $\begin{array}{r}\text { Calculation time } \\
(\text { hours })\end{array}$ \\
\hline \hline Fluent & 250 & $9.2 \times 10^{5}$ & 10 & $122(8$ cores $)$ \\
Gerris & var $86-2700$ & var $2 \times 10^{4}$ & var 2.0 & $28.8(1$ core $)$ \\
Slender jet & 100 & $7.7 \times 10^{3}$ & var 0.069 & $1.0(1$ core $)$ \\
\hline
\end{tabular}

Table 6.4: Benchmark case 2: Simulation resolution and calculation times. Same as table 6.2. 
drops, with a final momentum which is smaller than the average experimental momentum by $0.5 \mathrm{Ns}$. However for this case the total momentum of the initial case is also small compared to the average momentum determined around that time.

Next, we evaluate the movement of the head and the tail drops. The position of the maximal radii of the drops are traced and are plotted with the velocity in the drop at this position in Fig. 6.16. The movement of the head drop in the simulations match the experimental data very well. The velocity in the tail drops was found to increase up to the point where the drop breaks up. The velocities in the simulations start off at a smaller velocity than the average tail drop velocity from experiment, which is caused by the drop to drop variation of the velocity profile. In the simulations the decreasing steps in the tail velocity are recognized were the tail drop catches up with a satellite drop with lower velocity. We notice that the head and the tail drop velocity in the fluent simulation are both under-estimated through the drag in the Fluent model, which assumes quiescent air.

Next, the drop volumes after the breakup were evaluated. The PDF of the drop volume is plotted in Fig. 6.17, which indicated the distribution of the drop volume in time. Since the simulations only calculated a single drop formation process, the same drop volumes are the same calculated throughout the simulations. However, further breakup and coalesce causes changes in the calculated drop volumes. The satellite and tail drops were found to be 0.5 and $2 \mathrm{pL}$, respectively, which lie within the drop volumes range found in the experiments. The tail drop in both cases was found to coalesce with the satellite drops, and therefore increases to about $2.7 \mathrm{pL}$. This, however, is a typical drop volume which is not observed frequently in the experiments.

\subsubsection{Multi initial time steps simulations}

Thanks to the short calculation time of the slender jet model, multiple drop formation simulations could be run within a reasonable time. 30 simulations were run for different initial time steps, all starting with data obtained from experiment. The spread in the drop break-up processes results in a wider volume distribution than for the single initial condition evaluation. The drop volume histogram of these simulations is plotted in Fig. 6.18. This drop volume fraction per drop and time step follows the general trend of the drop volume breakup as found in the experiment. The simulations result in smaller head drops by approximately $0.5 \mathrm{pL}$. 


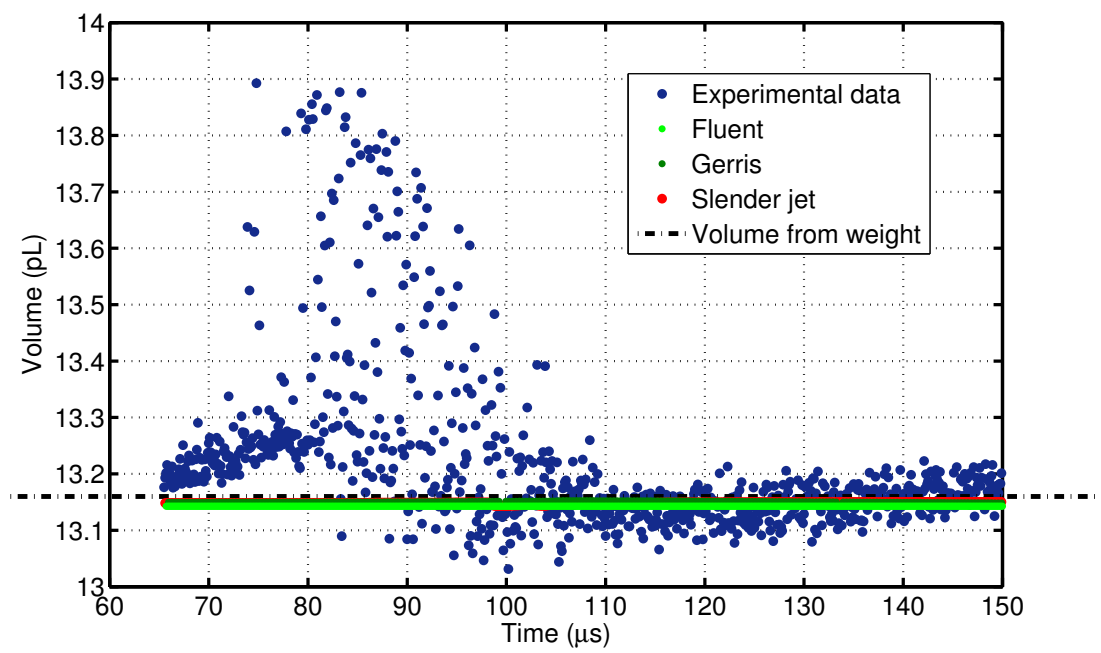

(a)

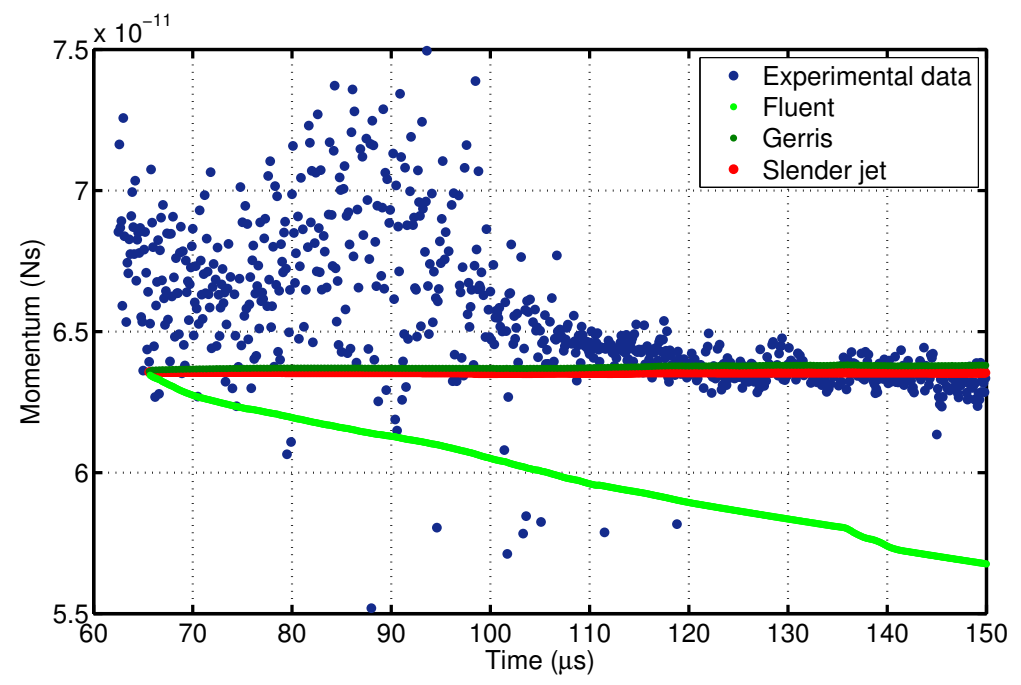

(b)

Figure 6.15: (a) Total volume of the experimental measurement and numerical models, the black line is the volume from the weighted volume. The drop volumes above $13.3 \mathrm{pL}$ are due to analysis difficulty of the perturbing tail. (b) Momentum of experimental data and numerical calculations. 


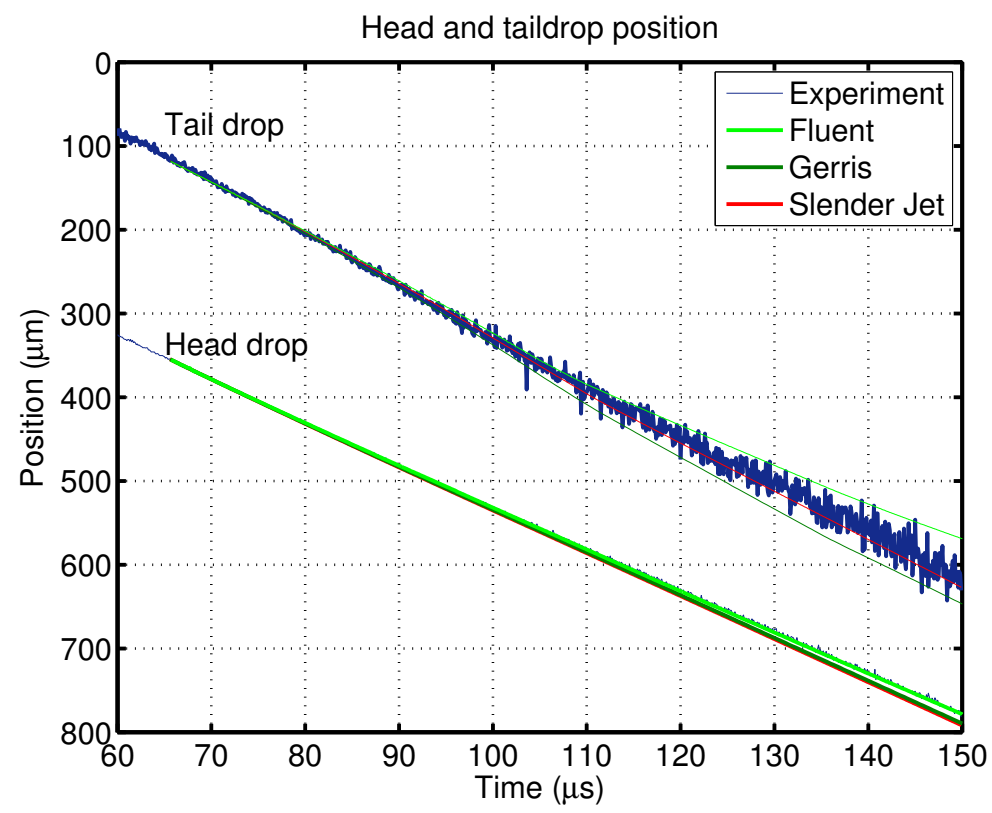

(a)

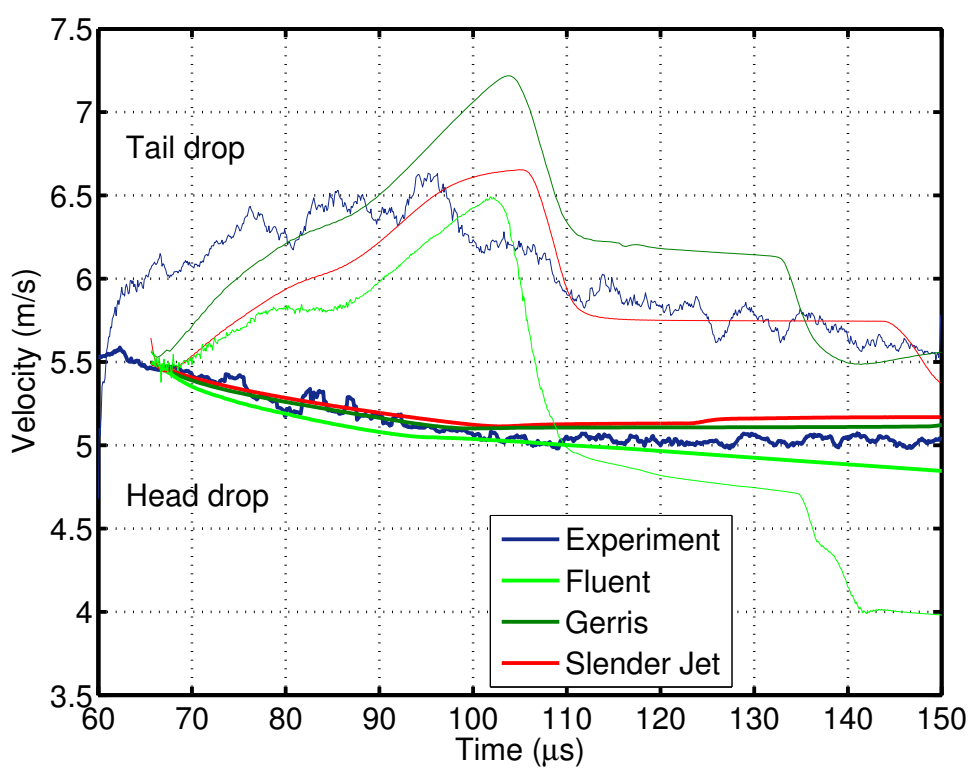

(b)

Figure 6.16: (a) Position comparison of the head and tail drops. (b) Velocity comparison of the head and tail drops. The experimental velocity is averaged over $2 \mu \mathrm{s}$. 


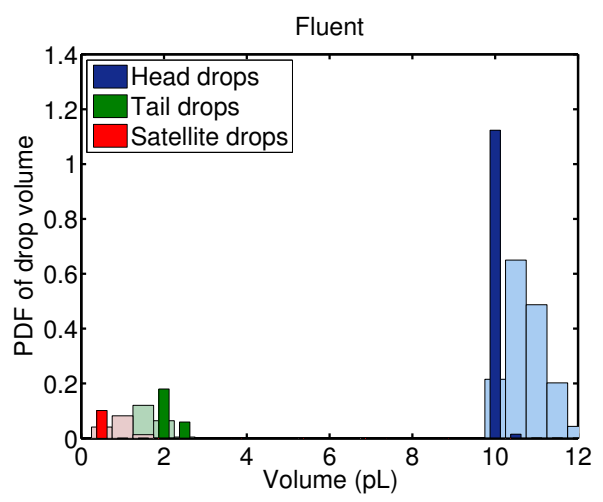

(a)

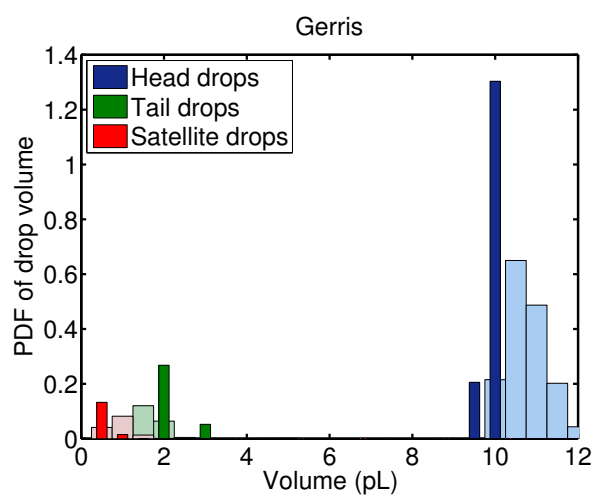

(c)

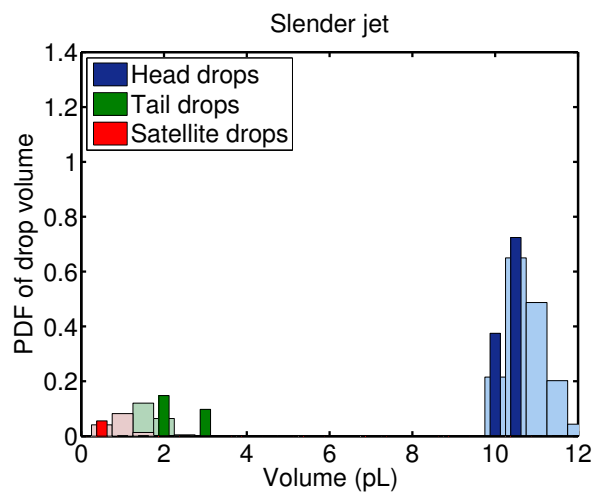

(e)

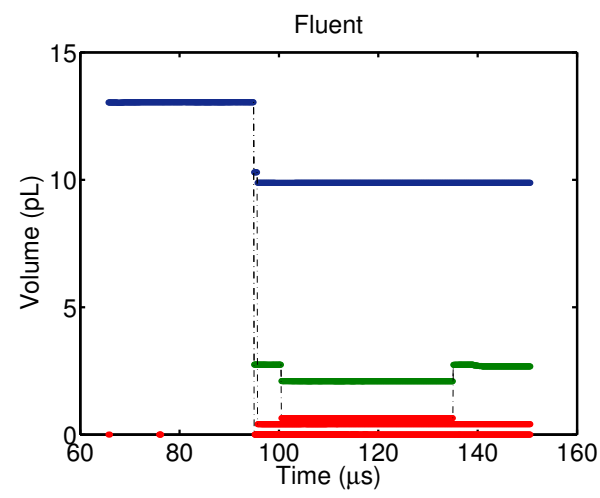

(b)

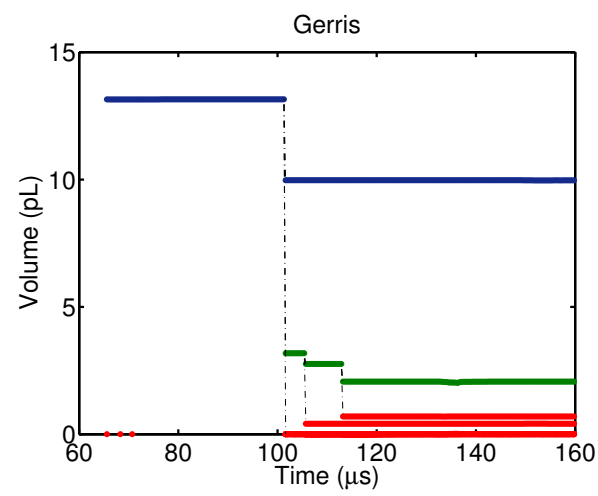

(d)

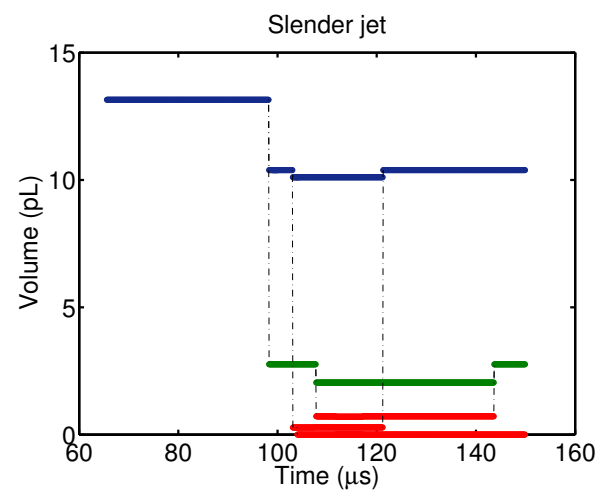

(f)

Figure 6.17: (a/c/e) PDF of drop volume of the three models using the same initial conditions (dark colors), after the breakup of the initial drop, of and experiment (light colors). (b/d/f) Evolution of the calculated drop volumes. The dotted lines are a guide to the eye for the drop volume reduction at break up of the drops. 


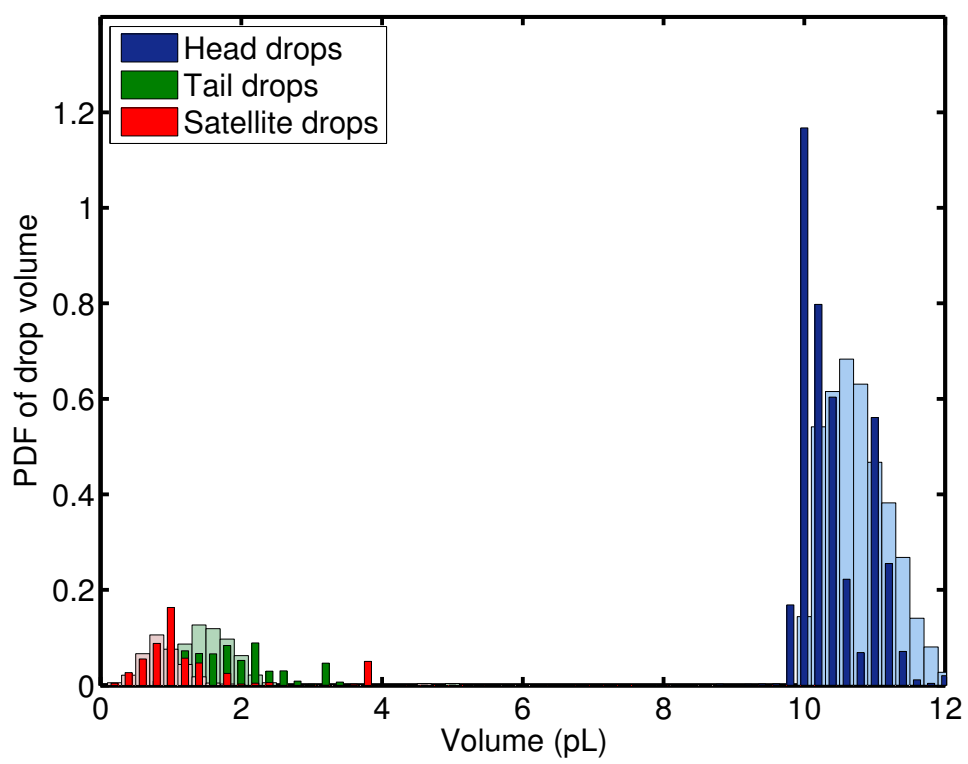

Figure 6.18: PDF of droplet volume of slender jet simulations (dark colors) for 30 simulations with different initial conditions and experiment (light colors). The drop volumes of all the time steps were taken into account in the time frame 95-150 $\mu \mathrm{s}$. 


\subsection{Conclusions \& Outlook}

In this studies we tested the results of CFD simulations of microdrop formation against experimental recordings. We used two VOF codes, namely Gerris and Fluent and a slender jet approximation to calculate two benchmark cases: a contracting drop and a drop which breaks up into smaller droplets. We find that all three models perform well for the used ink, which resembles a typical hot melt ink. Despite the differences in the used models, the predictions agree very well, and we have focussed on the subtle differences between the models.

For the first benchmark case the velocities in the head drop and tail drop were compared to the velocities in the experiments. The calculated head drop velocity and its the reduction due to the contraction was found to be equal to the velocity in the experiments. The velocity of the tail drop was calculated somewhat higher on average, causing a moment of contraction of $1-2 \mu$ s earlier than those measured in experiment.

For the second benchmark case we compared the volume distributions after the drop break up. The calculated volumes for all three models are within the experimentally measured range for the head drops, the tail drops and the satellite drops. Both VOF codes calculated an approximately $0.2 \mathrm{pL}$ smaller head volume than the slender jet approximation. In the Gerris simulation and the slender jet approximation a portion of the tail drop were calculated to have a volume between $2.7-2.8 \mathrm{pL}$ were calculated, which was were not observed in the experiments. However multiple initial time simulations were performed with the slender jet model. These show that the general drop breakup trend follows that found in the experiments.

We found that the slender jet model is significantly faster in CPU time, by a factor 20 or more in the second benchmark case. This was expected due to the 1dimensional nature of the model. This allows for larger parameter studies. We find that for axi symmetric drop formations, the slender jet model performs just as well as the tested VOF models (provided that air drag is not relevant), with very good mass and energy conservation, but the short calculation times involved are a strong benefit over the VOF codes. In the current benchmark cases the movement of the drop and the air was not consistently implemented and were run on different desktops. Therefore the calculation times of the VOF codes can not be compared directly.

It was found that with the inclusion of air in Gerris non-coalescence of the drops can occur. For the slender jet model, in which the fluid is always connected through a infinitesimally small filament, this non-coalescence can not happen. In the experimental data a large number of satellite drops next to one another were found, but most of these drops were found to move relatively towards one another with velocities between $0.5-1 \mathrm{~m} / \mathrm{s}$. Therefore coalescence is still expected for these cases. In some recordings, we could not detect a difference in velocity between the drops, indicating very slow coalescence or none at all. 
To perform this studies the drop boundary detection method for microdrops was improved using the average drop volume found from weighing more than $10^{7}$ drops. The improvement was found to be relevant for long elongated drops. The nonreproducible features of drop formation can however not be resolved by our experimental technique, e.g. the breakup of the tail into satellite drops and their subsequent coalescence. To resolve these features additional frames are needed, either with an additional iLIF setup, or with a high speed camera which can supply lower resolution recordings at later moments in time.

In our experiments with the drop trains the individual drops feel a reduced air drag due to air co-flowing with the drops. The drop velocity decrease though the air drag was found to be up to $7 \%$ during our typical experimental time of $100 \mu \mathrm{s}$, but becomes important for larger times. Simulations without air or in the reference frame of the drop underestimate the influence of this drag, whereas simulations with quiescent air do not take into account the reduction due to the co-flow. To account for this properly, the calculation of a drop stream is required with the inclusion of air in the model.

In this studies we find that all three models perform well for the viscous ink, which resemble a typical hot melt ink. Benchmarks for other ink types might show more variations between the models. The developed method was found to give highly detailed information on the differences between the models and is very suited for comparing the predictions of the numerous existing numerical drop formation codes.

\section{A Edge detection of the microdroplets}

When making an image of a microscopic object, the edge of the object is blurred over a small region. This is due to the finite wavelength of light, the optical transfer function of the optics and diffraction through the object. The determination of the exact boundary position therefore is challenging. Sensitivity of the position on the background intensity can be overcome by using the inflection point of the intensity over the boundary instead of a thresholding value. However position of the physical boundary however is not exactly at this inflection point, but slightly further into the drop, as was shown in Chapter 5. We improve this method by using the weigh of the drops. A single silicon oil drop of $10 \mathrm{pL}$ only weights $9.2 \mathrm{ng}$, therefor a large collection of about 10 million drops is required and the average drop mass of the collection is determined.

For the calibration spherical drops are used, since they have the most basic drop shape, the volumes of the drops are between 6 and $13 \mathrm{pL}$. To calibrate the offset of the edge, the drops are corrected over a range from 0 to $0.7 \mu \mathrm{m}$ and using interpolation the offset for which the drop volume matches the volume calculated from the weight is found. The offset from the inflection point to the boundary of the spheres 
is determined to be $0.41 \mu \mathrm{m}$, for the used lighting and optics.

Applying this correction on a droplet series of about 9.63 and $13.16 \mathrm{pL}$, which contracts from a elongated drop to a spherical drop, revels that this correction is not perfect over the entire part of elongated drops. The volume for a long elongated drop is about $0.3-1 \mathrm{pL}$ larger.

Observing the drop during contraction revels three main drop sections, the head drop, the tail and the tail drop, see Fig. 6.19. At time $37 \mu \mathrm{s}$, the drop has contracted and the head drop volume equals the total volume of the drop. For the different frames in the time series, the drop sections have been determined, and their volumes are plotted in the corresponding colors. The determined radius of the radius and length of the tail are plotted in Fig. 6.20. We now propose to use a different offset in the cylindrical tail from the inflection point to the physical boundary. The different offset for the cylindrical tail with respect to the one in the spherical drops, can be caused due to the smaller size of the tail, or due to a difference in diffraction. The radius of the tail can be calculated from the total measured volume, the volumes of the spherical segments and the length of the tail.

$$
\begin{aligned}
& V_{\text {total }}=V_{\text {Taildrop }}+V_{\text {Headdrop }}+V_{\text {Tail }} \\
& V_{\text {Tail }}=\pi R_{\text {Tail }}^{2} * L_{\text {Tail }} \\
& R_{\text {Tail }}=\sqrt{\frac{V_{\text {total }}-V_{\text {Taildrop }}-V_{\text {Headdrop }}}{\pi * L_{\text {Tail }}}},
\end{aligned}
$$

with $\mathrm{V}$ the volume of the drop segments, and $\mathrm{L}_{\text {Tail }}$ the length of the tail. $L_{\text {tail }}$ was measured in time during the contraction phase, see Fig.6.20. The total radius correction for the tail has been calculated to be $0.64 \mu \mathrm{m}$. Which is applied to both drop cases in the paper. For future references it would be nice to have an alternative check on the offset of the tail radius. One of the methods to measure this, is to use a micro rod with well known radius and with similar refractive index to the ink, for example glass. However it is hard to find and handle glass rods with long aspect ratios and radii of $1-3 \mu \mathrm{m}$. 


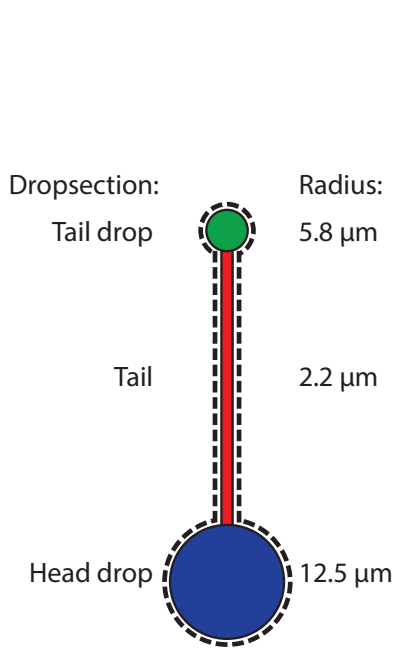

(a)

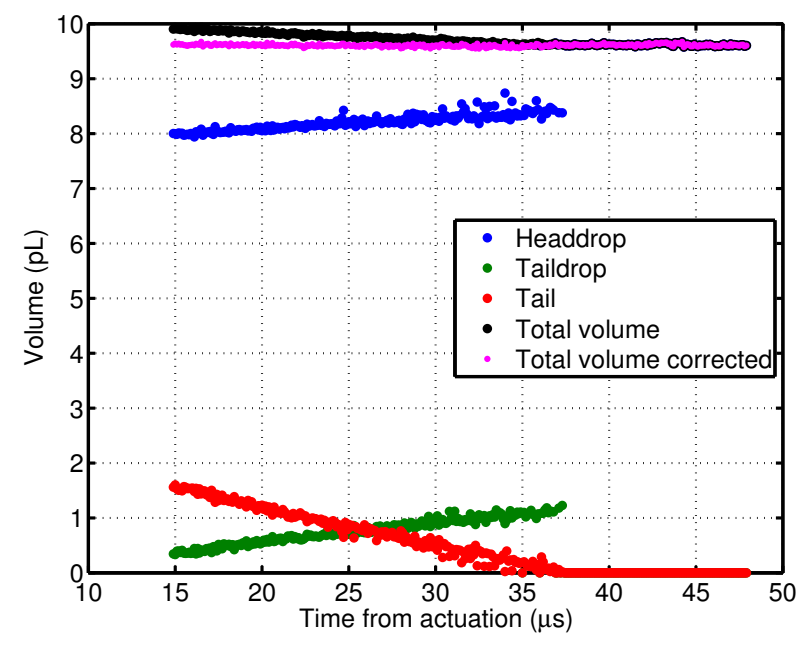

(b)

Figure 6.19: (a) The inkjet drops exist out of three different parts, the large volume spherical head drop, the cylindrical tail and the spherical tail drop. The radii of the drop sections are determined at time $25 \mu \mathrm{s}$. (b) The drop volume evolution of the uncorrected tailradius and tailradius corrected with $0.64 \mu \mathrm{m}$.

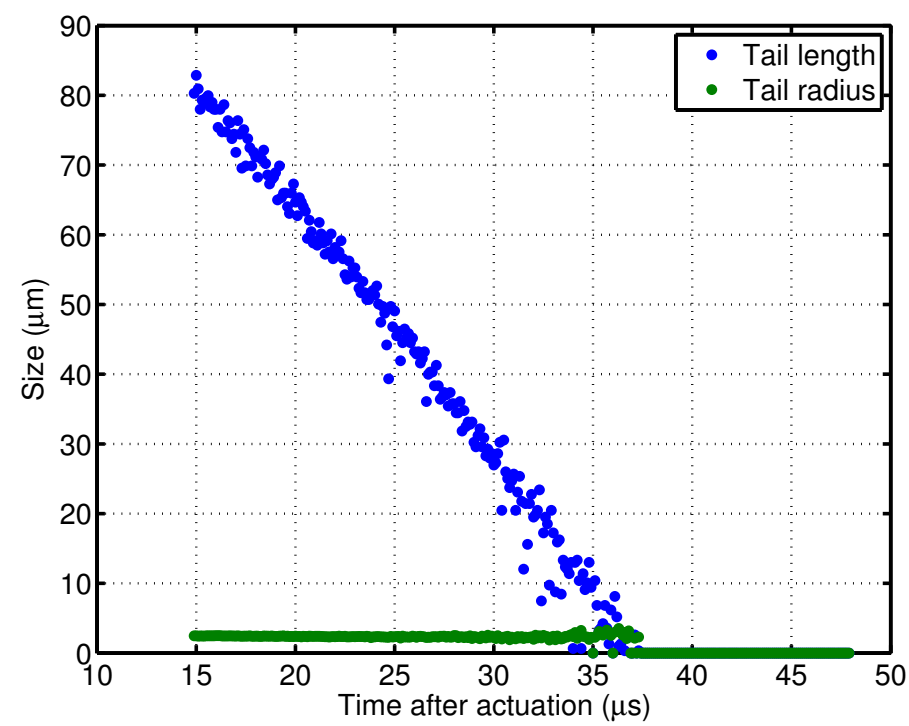

Figure 6.20: Measured tail length and radius. The tail contracts approximately linear at a constant radius. 


\section{B Drag reduction in drop trains}

In our experiments the droplets move in train formation, which drags along the air between the drops. We are interested in the effect of the co-flowing air on the drag of the individual drops, and whether this situations is resembled by a situation where there is no effective air drag on the drops or by a full drag situation of a single drop. The drag force on a spherical drop moving through air is given by

$$
F_{\text {drag }}=\rho C_{d} A v^{2} / 2,
$$

with A the frontal surface of the droplet, $\mathrm{v}$ the velocity of the drop, $\rho$ density of the air and $C_{d}$ is the Re number dependent drag coefficient. To account for the fact that $C_{d}$ is Reynolds number dependent, we use the description of Morrisson [140]

$$
C_{d}=\frac{24}{R e}+\frac{2.6\left(\frac{R e}{5.0}\right)}{1+\left(\frac{R e}{5.0}\right)^{1.52}}+\frac{0.411\left(\frac{R e}{263000}\right)^{-7.94}}{1+\left(\frac{R e}{263000}\right)^{-8.00}}+\left(\frac{R e^{0.8}}{461000}\right),
$$

which is valid for a range of Re between 0.1 and $10^{7}$.

The drops observed in our experiments move typically $100 \mu \mathrm{s}$ in the field of view of the camera. Therefor we are interested in the deceleration that drops experience in this time span.

Using eq. 6.2 and 6.3 we calculate the deceleration of microdrops over a timespan of $100 \mu \mathrm{s}$. The results for starting velocities ranging from 1 to $9 \mathrm{~m} / \mathrm{s}$ is plotted in Fig. 6.21 . In our experiments we have microdrops of about $10 \mathrm{pL}$ moving with $3 \mathrm{~m} / \mathrm{s}$, for a single drop the deceleration is expected to be approximately $2 \times 10^{3} \mathrm{~m} \mathrm{~s}^{2}$ in $100 \mu \mathrm{s}$.

First we analyze a experimental dataset of $6.0 \mathrm{pL}$ drops jetted at $10 \mathrm{kHz} .1000$ drops were recorded with a constant delay from the actuation. In the Fig. 6.22, the two successive jetted spherical drops are visible. Due to the $10 \mathrm{kHz}$ actuation frequency, the time from the moment of actuation for both drops is exactly $100 \mu$ s, i.e. the second drop is observed $100 \mu$ s later in time. We find the velocity using the double frame analysis method described in section 6.2.1. The first drop has an average velocity of $2.04 \mathrm{~m} / \mathrm{s}$ over 1000 recordings and the second drop moves with $1.91 \mathrm{~m} / \mathrm{s}$. This gives us a deceleration of $1.3 \times 10^{3} \mathrm{~m} / \mathrm{s}^{2}$ in $100 \mu \mathrm{s}$. From the drag theory we find find for the initial velocity: $\mathrm{Re}=2.75$ and $\mathrm{C}_{d}=9.95$, resulting in a deceleration $1.6 \times 10^{3} \mathrm{~m} / \mathrm{s}^{2}$ in $100 \mu \mathrm{s}$. For this actuation with $10 \mathrm{kHz}$ we find therefore a drag induced deceleration of the drop of $17 \%$.

Secondly a actuation of $9.6 \mathrm{pL}$ drops jetted at $20 \mathrm{kHz}$, see Fig. 6.23. For this actuation frequency the drops are packed more closely together. The drops were recorded with $100 \mathrm{~ns}$ delay steps, and combining all the spherical drops in the field of view, the drops can be traced over a time span of $160 \mu$ s. Figure 6.24 the velocities of the drops are plotted to the delay of the moment of actuation. From a linear fit through these velocities a deceleration of $1.3 \times 10^{3} \mathrm{~m} / \mathrm{s}^{2}$ in $100 \mu$ s was determined. 


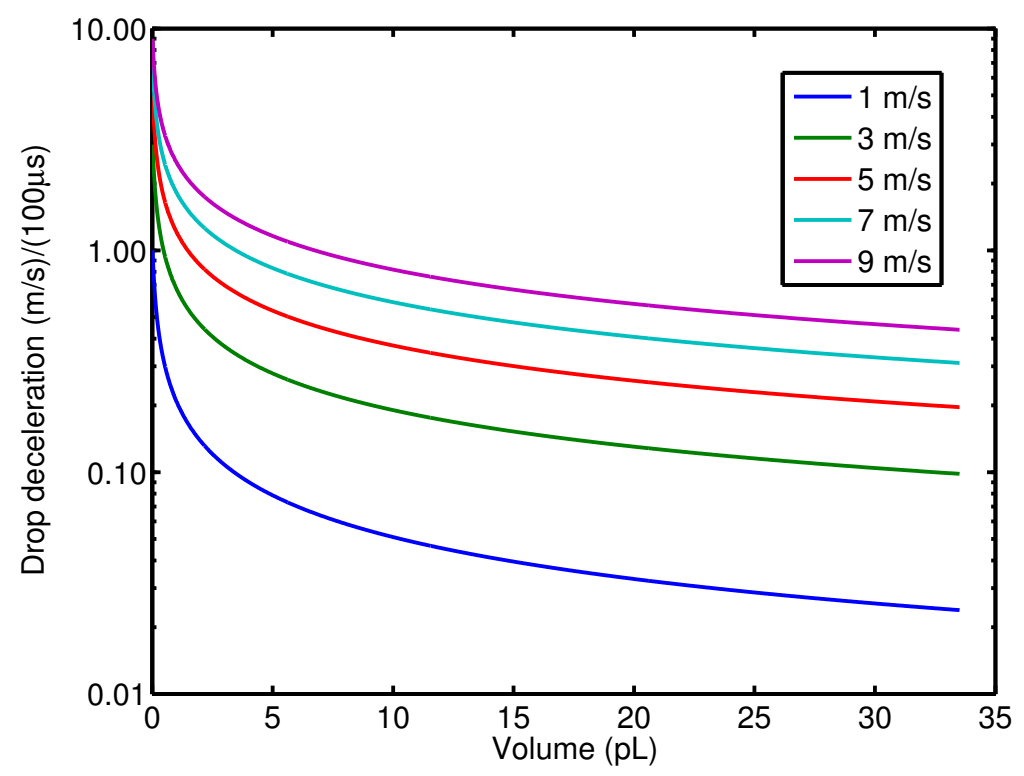

Figure 6.21: Deceleration of a spherical droplet in air over a $100 \mu$ s timespan, starting from different initial velocities. Small drops are stronger influenced than larger drops, very small drops reach the terminal velocity within the $100 \mu \mathrm{s}$. For larger drops also the velocity variation becomes less sensitive to the volume.

From the drag theory we find $\mathrm{Re}=4.79$ and $\mathrm{C}_{d}=6.51$, resulting in a deceleration of $1.95 \times 10^{3} \mathrm{~m} / \mathrm{s}^{2}$ in $100 \mu \mathrm{s}$. The reduction of air drag effect due to the co-flow of the air was found to is $33 \%$ for this case. Therefore the reduction of the air drag due to the co-flowing of air with the drops hardly causes a drag free situation for the recorded droplets. It is however to be expected that the closer the drops are moving together, the stronger the drag reduction effect becomes. 


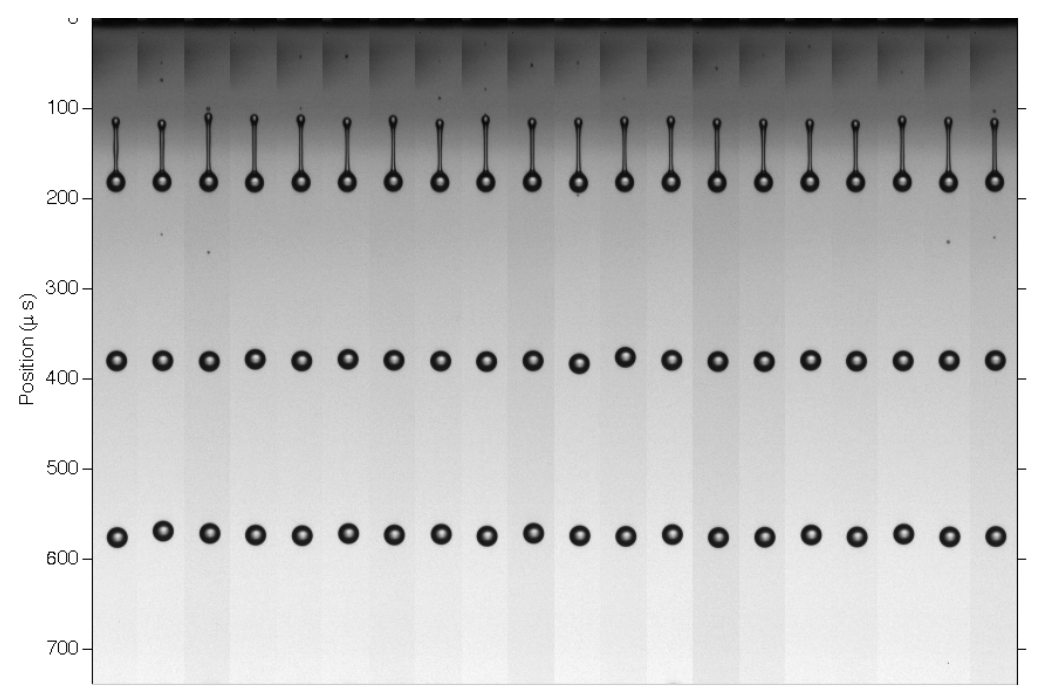

Figure 6.22: Actuation of $6.0 \mathrm{pL}$ droplets at $10 \mathrm{kHz}$. The images were all made with the same delay after the actuation. The delay between two successive drops is $100 \mu \mathrm{s}$. The bottom drops are decelerated by $1.3 \times 10^{3} \mathrm{~m} / \mathrm{s}^{2}$ in $100 \mu \mathrm{s}$.

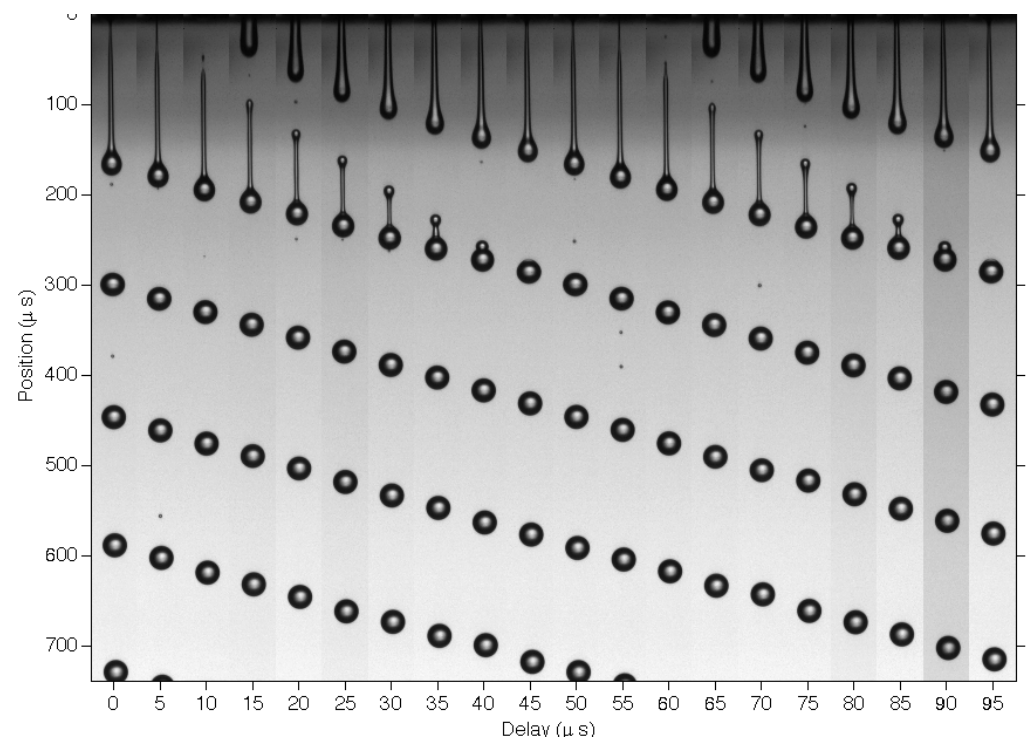

Figure 6.23: Actuation of $9.6 \mathrm{pL}$ droplets at $20 \mathrm{kHz}$. For over $160 \mu$ s droplets were traced. 


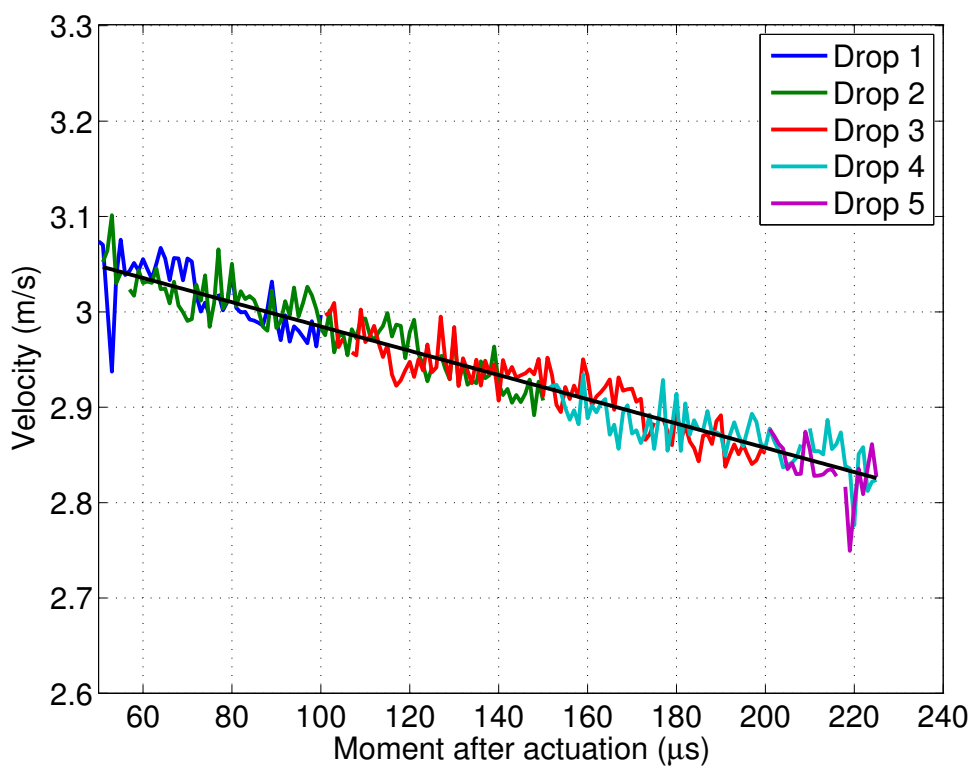

Figure 6.24: The velocities of the spherical drops as a function of the moment of actuation. From a linear fit over $160 \mu \mathrm{s}$ a deceleration of $1.3 \times 10^{3} \mathrm{~m} / \mathrm{s}^{2}$ in $100 \mu \mathrm{s}$ was determined. 


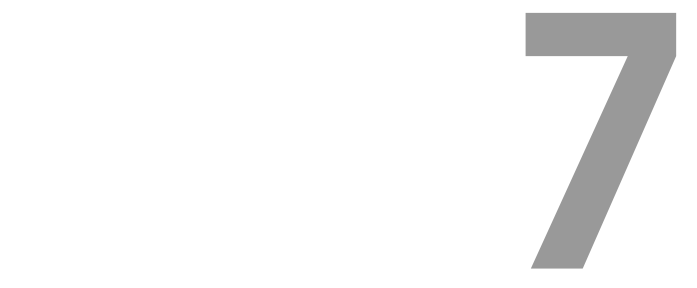

\section{Conclusions and outlook}

In this thesis we created and studied new methods, both numerical and experimental, to improve the understanding of small scale drop formation. These techniques and understanding are of great interest from an academic perspective, in addition to being highly relevant for inkjet printing industry. The topics in this thesis can be divided into two parts: the movement of the meniscus inside of the printhead nozzle (Chapter 2/3) and the dynamics within freely flying droplets (Chapter 4-6).

\subsection{Conclusions}

Several experimental techniques have been discussed to obtain information on the fluid inside the micro-nozzle of inkjet printheads, including velocity, shape and position of the meniscus, the volume in the nozzle and the pressure near the nozzle. Previous works which have deployed these techniques have been reviewed and variations were proposed. Next to the works found in literature, results of three new techniques for investigating the inkjet nozzle are presented: scanning LDV of the fluid meniscus, meniscus shape determination using a transparent nozzle and using tracer particles. With LDV the highest velocity resolution and spatial resolution (via numerical integration) is obtained. However it can only be applied in a small range, where the maximum velocity of the device is not exceeded and where the meniscus is not retracted too far into the nozzle. Inside of the nozzle the most insights on bubbles and meniscus shapes are obtained from the use of transparent nozzles, but optical refraction at the nozzle and fluid-air interface can make the interpretation challenging. 
Capacitive and piezoelectric sensing detect a direct electrical signal, which can be processed and acted upon during the jetting process and therefore these methods are highly suited for integration in a commercial print head.

Asymmetric features in inkjet droplet formation have multiple origins, which can be caused by different known physical asymmetries in the inkjet system. The possibility for the growth of asymmetric surface modes has been investigated in Chapter 3. The acceleration threshold for a Rayleigh-Taylor instability and a parametrically driven instability have been determined for a flat low-viscosity fluid meniscus in a cylindrical nozzle. We evaluated a $25 \mu \mathrm{m}$-radius nozzle Microdrop printhead for water. The acceleration of the meniscus inside the optically transparent Microdrop during actuation was determined and compared to the calculated instability thresholds. It was found that the acceleration threshold for the first symmetrical mode can be exceeded during drop formation for these instabilities. The observed acceleration of the main meniscus oscillation, however, only exceeded the theoretical acceleration thresholds for asymmetrical modes for a non-pinned meniscus. On the other hand, it is observed that for larger accelerations a deformation of the meniscus develops, which grows and eventually ejects a smaller droplet. For those cases where the acceleration thresholds were exceeded the time that this acceleration was experienced by the meniscus was of comparable or longer duration as compared to the theoretically determined growth times for both the instabilities.

The second part of the thesis was aimed at the studies of microdrop behavior in flight, and their evolution after pinch off from the ink meniscus in the nozzle. In Chapter 4 drop formation and the corresponding energy loss in ballistic energy conversion was investigated. The size distribution and velocity of the microdrops under different driving pressures and two different nozzle diameters were measured to gain insight in the energy conversion, through a quantification of the accompanying loss mechanisms. Drop formation was found to be irreproducible, therefore a double illumination technique was used with 1-2 $\mu$ s delay time in combination with high speed imaging. The energy conversion was found to take place in two stages. In the first stage pressure is converted to kinetic energy and surface energy of the microdrops; the main loss in this phase is viscous dissipation and modification of the surface energy while the drop is forced through the narrow nozzle orifice. In the second stage the kinetic energy is converted into electric energy and where the main loss mechanism was found to be air drag of the microdrops. For the $10 \mu \mathrm{m}$ nozzle the energy loss was $\sim 52 \%$ for the first phase and typically $\sim 17 \%$ of the energy was lost in the second phase. A better energy conversion was found for larger nozzles. A maximal energy conversion of $36 \%$ was measured for a $10 \mu \mathrm{m}$ diameter nozzle and an efficiency of $48 \%$ for a $30 \mu \mathrm{m}$ diameter nozzle, which is a major improvement over the highest conversion efficiency reported until now, which was $10 \%$ at maximum. 
A novel method for quantitative analysis of the internal flow of drop formation in flight was also presented. Droplets are imaged using illumination by laser-induced fluorescence (ILIG), with nanoseconds illumination time, allowing detailed images without motion blur. Two images of a single droplet are imaged using a dual frame setup. The images are analyzed and the droplet volume calculated. Here we find that a droplet to droplet variation of maximal $0.13 \mathrm{pL}$ and the absolute accuracy in the volume estimation is approximately $17 \mathrm{fL}$. This shows that, to accurately extract the droplet dynamics, the reproducibility is not good enough and at least two images of a single droplet are required. We demonstrate a method to extract the velocity within the droplet, and validate the method with a numerical model. Here we find excellent agreement, confirming the applicability of the method.

The drop boundary detection method for microdrops was improved using the average drop volume found from weighing more than $10^{7}$ drops. The drop radius and velocity profile are determined when the droplet pinches off from the nozzle. Using this recording as the initial condition in the numerical drop formation models, the accuracy of the simulations could be compared with respect to the time evolution of droplet. For this study a slender jet model, Gerris and Fluent were used. Despite the differences in the models, the predictions agree very well, and we have focussed on the subtle differences between the models. For the first case the moment of drop contraction in the models was found to be similar, but $1-2 \mu$ s earlier than those found in experiment. We found that the slender jet model calculation time is significantly shorter, by a factor 20 or more. This is expected due to the small set of equations and the 1-dimensional nature of the model. Thus the slender jet model allows for larger parameter studies. The inclusion of air in the model leads to the possibility of non-coalescence of drops, for which some hints were found in experiments. We find that for axi-symmetric drop formations, the slender jet model, performs just as well as the tested VOF model, with very good mass and energy conservation. The short calculation times involved, make the slender jet model the preferred numerical model over over the VOF codes, when air influences are negligible. 


\subsection{Outlook}

The high-speed recordings using iLIF are a major improvement for a series of microfluidic experiments at nanosecond timescales. To obtain time resolved information, however, highly reproducible systems are required. The use of double-frame recordings allows for two snapshots of the same event and broadens the range of applications. The analysis system developed in Chapter 5, for example, is highly suited for a further study of the small scale drop formation in greater detail. Potential detrimental effects of the ink in the nozzle, such as a retracting meniscus or flooding of the nozzle plates can be counteracted using adaptions to the standard actuation block pulse. Analysis of the velocity profile inside the drops allows for a study of the effect for more sophisticated actuation pulses on the drop formation. The inspection of a transparent printhead nozzle, as concluded in Chapter 2, is highly promising to reveal potential bubble entrapment and (asymmetrical) motion of the meniscus. Modern MEMS printheads are manufactured using fused silica, which is intransparent to visible light. To overcome this problem, the nozzle can be inspected using infrared light of a wavelength near $1.4 \mu \mathrm{m}$, for which fused silica is transparent. This novel approach can reveal the behavior of the meniscus during jetting within an industrial production nozzle with a loss in resolution by only a factor of 2 . The formation of asymmetric surface modes at the inkjet meniscus has been supported from numerical analysis, albeit by the use of an oversimplification of the predictions of the RayleighTaylor and parametrically driven instabilities. The models can be extended to include the full effects of viscosity and pinning behavior at the nozzle walls, such as a dynamic contact line, to obtain better predictions for higher frequency printheads. Alternatively, it would be interesting to experimentally verify at what particular surface mode, the drop formation becomes asymmetrical. This would require a specialized set up to drive surface modes at the meniscus, for example by shaking the printhead near natural frequency of the interface, using a shaker. Finally, to to observe the asymmetrical features of drop formation, which typically is not observed or are out of focus using single camera backlight illumination techniques, the setup can be extended using an orthogonal double camera setup. 


\section{Bibliography}

[1] C. Kullmann, N. C. Schirmer, M.-T. Lee, S. H. Ko, N. Hotz, C. P. Grigoropoulos, and D. Poulikakos, "3D micro-structures by piezoelectric inkjet printing of gold nanofluids", J. Micromech. Microeng. 22, 055022 (2012).

[2] M. Vaezi, H. Seitz, and S. Yang, "A review on 3D micro-additive manufacturing technologies”, J. Appl. Mech. 67, 1721-1754 (2012).

[3] I. M. Hutchings and G. D. Martin, eds., Inkjet Technology for Digital Fabrication (John Wiley \& Sons, Ltd) (2013).

[4] H. Wijshoff, "The dynamics of the piezo inkjet printhead operation", Phys. Rep. 491, 77-177 (2010).

[5] J. R. Castrejon-Pita, W. Baxter, J. Morgan, S. Temple, G. D. Martin, and I. M. Hutchings, "Future, opportunities and challenges of inkjet technologies", Atomization Spray 23, 541-565 (2013).

[6] C. Ru, J. Luo, S. Xie, and Y. Sun, "A review of non-contact micro-and nanoprinting technologies”, J. Micromech. Microeng. 24, 053001 (2014).

[7] J. Eggers, "Drop formation-an overview", Z. Angew. Math. Mech 85, 400410 (2005).

[8] J. L. Vaught, F. L. Cloutier, D. K. Donald, J. D. Meyer, C. A. Tacklind, and H. H. Taub, “Thermal ink jet printer”, US 4,490,728 (1984).

[9] S. I. Zoltan, "Pulsed droplet ejecting system”, US patent 3,683,212 A (1972).

[10] A. van der Bos, A. Zijlstra, E. Gelderblom, and M. Versluis, "iLIF: illumination by Laser-Induced Fluorescence for single flash imaging on a nanosecond timescale”, Exp. Fluids 51, 1283-1289 (2011).

[11] J. Eggers, “Theory of Drop Formation”, Phys. Fluids 7, 941-953 (1995).

[12] T. Driessen and R. Jeurissen, "A regularised one-dimensional drop formation and coalescence model using a total variation diminishing (TVD) scheme on a single Eulerian grid", Int. J. Comput. Fluid Dyn. 25, 333-343 (2011). 
[13] I. Endo, Y. Sato, S. Saito, T. Nakagiri, and S. Ohno, "Bubble jet recording method and apparatus in which a heating element generates bubbles in a liquid flow path to project droplets", US Patent 4,723,129 (1988).

[14] R. Jeurissen, A. van der Bos, H. Reinten, M. van den Berg, H. Wijshoff, J. de Jong, M. Versluis, and D. Lohse, "Acoustic measurement of bubble size in an inkjet printhead", J. Acoust. Soc. Am. 126, 2184-2190 (2009).

[15] J. Dijksman, "Hydro-acoustics of piezoelectrically driven ink-jet print heads", Flow Turbul. Combust. 61, 211-237 (1998).

[16] D. Bogy and F. Talke, "Experimental and theoretical study of wave propagation phenomena in drop-on-demand ink jet devices", IBM J. Res. Dev. 28, 314-321 (1984).

[17] H. C. Wu, T. R. Shan, W. S. Hwang, and H. J. Lin, "Study of micro-droplet behavior for a piezoelectric inkjet printing device using a single pulse voltage pattern", Mater. Trans. 45, 1794-1801 (2004).

[18] Flow Science, Inc., "FLOW-3D”, http://www.flow3d.com.

[19] J. Eggers, "Nonlinear dynamics and breakup of free-surface flows", Rev. Mod. Phys. 69, 865-930 (1997).

[20] R. Badie and D. F. de Lange, "Mechanism of drop constriction in a drop-ondemand inkjet system”, Proc. R. Soc. A 453, 2573-2581 (1997).

[21] N. Link and R. Semiat, "Ink drop motion in wide-format printers: I. Drop flow from Drop-On-Demand (DOD) printing heads", Chem. Eng. Process. 48, 68-83 (2009).

[22] H. Wijshoff, "Drop formation mechanisms in piezo-acoustic inkjet", NSTINanotech 3, 448 - 451 (2007).

[23] J. Q. Feng, "A general fluid dynamic analysis of drop ejection in drop-ondemand ink jet devices", J. Imaging Sci. Technol. 46, 398-408 (2002).

[24] O. G. Harlen and J. R. Castrejón-Pita, "Asymmetric Detachment from Angled Nozzles Plates in Drop-on Demand Inkjet Printing”, J. Imaging Sci. Technol. 29, 277-280 (2013).

[25] S. Popinet, "Gerris: a tree-based adaptive solver for the incompressible Euler equations in complex geometries", J. Comput. Phys. 190, 572-600 (2003).

[26] M. Massucci, P. Boltryk, T. Tuladhar, and P. Drury, "From Ink Bottle to Ink Drop: The Flow Environment in an Inkjet Printhead", in NIP27, 54-58 (2011). 
[27] A. van der Bos, M.-J. van der Meulen, T. Driessen, M. van den Berg, H. Reinten, H. Wijshoff, M. Versluis, and D. Lohse, "Velocity Profile inside Piezoacoustic Inkjet Droplets in Flight: Comparison between Experiment and $\mathrm{Nu}-$ merical Simulation”, Phys. Rev. Applied 1, 014004 (2014).

[28] J. R. Castrejón-Pita, N. F. Morrison, O. G. Harlen, G. D. Martin, and I. M. Hutchings, "Experiments and Lagrangian simulations on the formation of droplets in drop-on-demand mode", Phys. Rev. E 83, 036306 (2011).

[29] G. D. Martin, J. R. Castrejón-Pita, and I. M. Hutchings, "Holographic Measurement of Drop-on-Demand Drops in Flight", in NIP27, 620-623 (2011).

[30] R. J. Adrian and J. Westerweel, Particle Image Velocimetry (Cambridge University Press) (2011).

[31] C. D. Meinhart and H. S. Zhang, "The flow structure inside a microfabricated inkjet printhead", J. Microelectromech. Syst. 9, 67-75 (2000).

[32] J. R. Castrejón-Pita, S. D. Hoath, A. A. Castrejón-Pita, N. F. Morrison, W.K. Hsiao, and I. M. Hutchings, "Time-Resolved Particle Image Velocimetry within the Nozzle of a Drop-on-Demand Printhead", J. Imaging Sci. Technol. 56, 1-6 (2012).

[33] C. Rembe, G. Siegmund, H. Steger, and M. Wörtge, "Measuring mems in motion by laser doppler vibrometry", in Optical inspection of microsystems, 1st edn. CRC/Taylor \& Francis, Boca Raton, edited by W. Osten, 245-292 (CRC Press) (2006).

[34] P. Castellini, M. Martarelli, and E. P. Tomasini, "Laser Doppler Vibrometry: Development of advanced solutions answering to technology's needs", Mech. Syst. Signal Pr. 20, 1265-1285 (2006).

[35] C. C. Poon and F. C. Lee, "Measurements of Refill Time in Thermal Ink-Jet Heads", in NIP7, 130-139 (1991).

[36] C. C. Poon and F. C. Lee, "Crosstalk Study of a Thermal Inkjet Print Head From Real-Time Drop Size and Velocity Measurements", in NIP9, 391-398 (1993).

[37] M. Seo, T. Tsukamoto, and Y. Norikane, "A Study on Drying Process of Ink at the Inkjet Nozzle Using a Laser Doppler Vibrometer", in NIP27, 347-350 (2011). 
[38] J. Wei, C. Yue, M. van der Velden, Z. L. Chen, Z. W. Liu, K. A. A. Makinwa, and P. M. Sarro, "Design, fabrication and characterization of a femto-farad capacitive sensor for pico-liter liquid monitoring", Sensor Actuat. A-Phys. 162, 406-417 (2010).

[39] J. Wei, C. Yue, G. Zhang, P. Sarro, and J. Dijksman, "Monitoring of meniscus motion at nozzle orifice with capacitive sensor for inkjet applications", in Sensors, 2012 IEEE, 1-4 (2012).

[40] J. Wei, P. M. Sarro, and T. C. Duc, "A piezoresistive sensor for pressure monitoring at inkjet nozzle", in Sensors, 2010 IEEE, 2093-2096 (2010).

[41] J. de Jong, G. de Bruin, H. Reinten, M. van den Berg, H. Wijshoff, M. Versluis, and D. Lohse, "Air entrapment in piezo-driven inkjet printheads", J. Acoust. Soc. Am. 120, 1257-1265 (2006).

[42] H. Wijshoff, "Structure-and fluid-dynamics in piezo inkjet printheads", Ph.D. thesis, University of Twente (2008).

[43] W.-K. Hsiao, S. D. Hoath, G. D. Martin, and I. M. Hutchings, "Jetting, InNozzle Meniscus Motion and Nozzle-Plate Flooding in an Industrial Drop-onDemand Print Head", in NIP27, 66-69 (2011).

[44] J. R. Castrejón-Pita, S. D. Hoath, and I. M. Hutchings, "Velocity Profiles in a Cylindrical Liquid Jet by Reconstructed Velocimetry", J. Fluid. Eng-T. ASME 134, 011201 (2012).

[45] D. Liu, S. V. Garimella, and S. T. Wereley, "Infrared micro-particle image velocimetry in silicon-based microdevices", Exp. Fluids 38, 385-392 (2005).

[46] A. van der Bos, T. Segers, R. Jeurissen, M. van den Berg, H. Reinten, H. Wijshoff, M. Versluis, and D. Lohse, "Infrared imaging and acoustic sizing of a bubble inside a micro-electro-mechanical system piezo ink channel", J. Appl. Phys. 110, 034503 (2011).

[47] K. Fukuda, T. Sekine, D. Kumaki, and S. Tokito, "Profile control of inkjet printed silver electrodes and their application to organic transistors", ACS Appl. Mater. Interfaces 5, 3916-3920 (2013).

[48] N. Chilton, "Printed circuit board fabrication", in Inkjet Technology for Digital Fabrication, 183-206 (John Wiley \& Sons, Ltd) (2013).

[49] H. C. Ki, S. H. Kim, D.-G. Kim, T.-U. Kim, S.-G. Kim, K.-J. Hong, and S.Y. So, "Printing method for organic light emitting device lighting", in SPIE OPTO, 86221J-86221J (2013). 
[50] A. Teichler, R. Eckardt, S. Hoeppener, C. Friebe, J. Perelaer, A. Senes, M. Morana, C. J. Brabec, and U. S. Schubert, "Combinatorial screening of polymer:fullerene blends for organic solar cells by inkjet printing", Advanced Energy Materials 1, 105-114 (2011).

[51] M. Jung, J. Kim, J. Noh, N. Lim, C. Lim, G. Lee, J. Kim, H. Kang, K. Jung, A. D. Leonard, J. M. Tour, and G. Cho, "All-Printed and Roll-to-Roll-Printable 13.56-MHz-Operated 1-bit RF Tag on Plastic Foils", IEEE Trans. Electron Devices 57, 571-580 (2010).

[52] B. O'Neill, “Three-dimensional digital fabrication", in Inkjet Technology for Digital Fabrication, 325-342 (John Wiley \& Sons, Ltd) (2013).

[53] D. B. Wallace, "Manufacturing of micro-electro-mechanical systems (mems)", in Inkjet Technology for Digital Fabrication, 141-158 (John Wiley \& Sons, Ltd) (2013).

[54] C. A. Curcio, K. R. Sloan, R. E. Kalina, and A. E. Hendrickson, "Human photoreceptor topography”, J. Comp. Neurol. 292, 497-523 (1990).

[55] M. Caironi, E. Gili, and H. Sirringhaus, "Ink-jet printing of downscaled organic electronic devices", in Organic Electronics II, 281-326 (Wiley-VCH Verlag GmbH \& Co. KGaA) (2012).

[56] Y. Wang, J. Bokor, and A. Lee, "Maskless lithography using drop-on-demand inkjet printing method", in Microlithography 2004, 628-636 (SPIE) (2004).

[57] C. Menzel, P. Hoisington, and A. Bibl, "Mems solutions for precision microfluidic dispensing application", in NIP20, 169-175 (2004).

[58] A. L. Brady, M. M. McDonald, S. N. Theriault, and B. Smith, "The impact of silicon mems on the future of ink jet printhead design and performance", in NIP21, 264-267 (2005).

[59] S. Berger, R. Burr, and D. Tence, "Method and apparatus for producing dot size modulated ink jet printing", (1997), US Patent 5,689,291.

[60] A. U. Chen and O. A. Basaran, "A new method for significantly reducing drop radius without reducing nozzle radius in drop-on-demand drop production", Phys. Fluids 14, L1 (2002).

[61] M. W. Lee, D. K. Kang, N. Y. Kim, H. Y. Kim, S. C. James, and S. S. Yoon, “A study of ejection modes for pulsed-DC electrohydrodynamic inkjet printing", J. Aerosol Sci. 46, 1-6 (2012). 
[62] I. M. Hutchings and G. D. Martin, "Introduction to Inkjet Printing for Manufacturing”, in Inkjet Technology for Digital Fabrication, 1-20 (John Wiley \& Sons, Ltd) (2013).

[63] K. Suganuma, Introduction to printed electronics (Springer, New York) (2014).

[64] N. Link, S. Lampert, R. Gurka, A. Liberzon, G. Hetsroni, and R. Semiat, "Ink drop motion in wide-format printers: II. Airflow investigation", Chem. Eng. Process. 48, 84-91 (2009).

[65] W.-K. Hsiao, S. D. Hoath, G. D. Martin, and I. M. Hutchings, “Aerodynamic effects in ink-jet printing on a moving web”, in NIP28, 412-415 (2012).

[66] A. A. Khalate, X. Bombois, R. Babuška, H. Wijshoff, and R. Waarsing, "Performance improvement of a drop-on-demand inkjet printhead using an optimization-based feedforward control method", Control Eng. Prac. 19, 771 -781 (2011).

[67] A. K. Mogalicherla, S. Lee, P. Pfeifer, and R. Dittmeyer, "Drop-on-demand inkjet printing of alumina nanoparticles in rectangular microchannels", Microfluidics Nanofluidics (2013).

[68] L. T. Creagh and M. McDonald, "Design and Performance of Inkjet Print Heads for Non-Graphic-Arts Applications", MRS Bulletin 28, 807-811 (2011).

[69] M. M. Fyrillas and A. J. Szeri, "Dissolution or growth of soluble spherical oscillating bubbles”, J. Fluid Mech. 277, 381-407 (1994).

[70] T. Leighton, The Acoustic Bubble (Academic Press) (1997).

[71] D. H. Kwon and S. J. Lee, "Axis-switching of non-axisymmetric microdroplet ejected from inkjet with an elliptical nozzle”, Phys. Fluids 23, 051703 (2011).

[72] J. R. Castrejón-Pita, G. D. Martin, and I. M. Hutchings, "Experimental Study of the Influence of Nozzle Defects on Drop-on-Demand Ink Jets", J. Imaging Sci. Technol. 55, 040305 (2011).

[73] Rayleigh, "Investigation of the character of the equilibrium of an incompressible heavy fluid of variable density", Proc. R. Soc. A s1-14, 170-177 (1882).

[74] G. Taylor, "The instability of liquid surfaces when accelerated in a direction perpendicular to their planes. I", Proc. R. Soc. A 201, 192-196 (1950). 
[75] M. Faraday, "On a peculiar class of acoustical figures; and on certain forms assumed by groups of particles upon vibrating elastic surfaces", Phil. Trans. R. Soc. 299-340 (1831).

[76] J. F. Dijksman and A. Pierik, "Dynamics of piezoelectric print-heads", in Inkjet Technology for Digital Fabrication, 45-86 (John Wiley \& Sons, Ltd) (2013).

[77] A. Royon-Lebeaud, E. J. Hopfinger, and A. Cartellier, "Liquid sloshing and wave breaking in circular and square-base cylindrical containers", J. Fluid Mech. 577, 467 (2007).

[78] S. Chandrasekhar, Hydrodynamic and Hydromagnetic Stability, International Series of Monographs on Physics (Clarendon Press) (1970).

[79] J.-L. Achard and S.-C. Georgescu, "Quasi-steady formation of bubbles and drops viewed as processes that break bifurcation", J. Eng. Math. 51, 147-164 (2005).

[80] S. Ciliberto and J. P. Gollub, "Phenomenological Model of Chaotic Mode Competition in Surface-Waves", Nuovo Cimento D. 6, 309-316 (1985).

[81] T. B. Benjamin and F. Ursell, "The stability of the plane free surface of a liquid in vertical periodic motion”, Proc. R. Soc. A 225, 505-515 (1954).

[82] W. S. Edwards and S. Fauve, "Patterns and quasi-patterns in the Faraday experiment", J. Fluid Mech. 278, 123-148 (1994).

[83] J. Bechhoefer, V. Ego, S. Manneville, and B. Johnson, "An experimental study of the onset of parametrically pumped surface waves in viscous fluids", J. Fluid Mech. 288, 325-350 (1995).

[84] K. Kumar and L. S. Tuckerman, "Parametric instability of the interface between two fluids", J. Fluid Mech. 279, 49-68 (1994).

[85] M. Versluis, "High-speed imaging in fluids", Exp. Fluids 54, 1-35 (2013).

[86] A. W. Bright and B. Makin, "Modern electrostatic generators", Contemp. Phys. 10, 331-353 (1969).

[87] N. Tesla, "Possibilities of electro-static generators", Sci. Am. 150, 132-165 (1934).

[88] Y. Xie, X. Wang, J. Xue, K. Jin, L. Chen, and Y. Wang, "Electric energy generation in single track-etched nanopores", Appl. Phys. Lett. 93 (2008). 
[89] Y. Xie, J. D. Sherwood, L. Shui, A. van den Berg, and J. C. Eijkel, "Strong enhancement of streaming current power by application of two phase flow", Lab Chip 11, 4006-4011 (2011).

[90] A. M. Duffin and R. J. Saykally, "Electrokinetic hydrogen generation from liquid water microjets", J. Phys. Chem. C 111, 12031-12037 (2007).

[91] A. M. Duffin and R. J. Saykally, "Electrokinetic power generation from liquid water microjets", J. Phys. Chem. C 112, 17018-17022 (2008).

[92] Y. Xie, D. Bos, L. J. de Vreede, H. L. de Boer, M.-J. van der Meulen, M. Versluis, A. J. Sprenkels, A. van den Berg, and J. C. Eijkel, "High-efficiency ballistic electrostatic generator using microdroplets", Nat. Commun. 5 (2014).

[93] G. M. Whitesides, "The origins and the future of microfluidics", Nature 442, 368-373 (2006).

[94] T. M. Squires and S. R. Quake, "Microfluidics: Fluid physics at the nanoliter scale", Rev. Mod. Phys. 77, 977-1026 (2005).

[95] F. A. Morrison Jr. and J. F. Osterle, "Electrokinetic Energy Conversion in Ultrafine Capillaries", J. Chem. Phys. 43, 2111-2115 (1965).

[96] J. F. Osterle, "Electrokinetic Energy Conversion”, J. Appl. Mech. 31, 161-164 (1964).

[97] S. Pennathur, J. C. T. Eijkel, and A. van den Berg, "Energy conversion in microsystems: is there a role for micro/nanofluidics?", Lab Chip 7, 1234-1237 (2007).

[98] M. Faubel and B. Steiner, "Strong bipolar electrokinetic charging of thin liquid jets emerging from $10 \mu \mathrm{m}$ ptir nozzles", Ber. Bunsenges. Phys. Chem. 96, 1167-1172 (1992).

[99] J. M. Schneider, "Stability of an Electrified Liquid Jet", J. Appl. Phys. 38, 2599-2605 (1967).

[100] H. E. White, Modern College Physics, By Harvey E. White. (New York, Van Nostrand) (1949).

[101] W. van Hoeve, S. Gekle, J. H. Snoeijer, M. Versluis, M. P. Brenner, and D. Lohse, "Breakup of diminutive Rayleigh jets", Phys. Fluids 22, 122003 (2010).

[102] R. A. Sampson, “On Stokes's current function”, Phil. Trans. R. Soc. A 182, 449-518 (1891). 
[103] S. Sisavath, X. D. Jing, C. C. Pain, and R. W. Zimmerman, "Creeping flow through an axisymmetric sudden contraction or expansion", J. Fluid. Eng-T. ASME 124, 273-278 (2002).

[104] J. H. Lienhard, "Velocity Coefficients for Free Jets From Sharp-Edged Orifices", J. Fluid. Eng-T. ASME 106, 13-17 (1984).

[105] E. F. Brater, H. W. King, J. E. Lindell, and C. Y. Wei, Handbook of hydraulics for the solution of hydraulic engineering problems, Civil Engineering (McGraw-Hill) (1996).

[106] F. A. Morrison, An Introduction to Fluid Mechanics (Cambridge University Press) (2013).

[107] F. W. Peek, Dielectric phenomena in high voltage engineering (McGraw-Hill Book Company, Incorporated) (1929).

[108] H. Dong, W. W. Carr, and J. F. Morris, "Visualization of drop-on-demand inkjet: Drop formation and deposition”, Rev. Sci. Instrum. 77, 085101 (2006).

[109] G. D. Martin, S. D. Hoath, and I. M. Hutchings, "Inkjet printing - the physics of manipulating liquid jets and drops", J. Phys. Conf. Ser. 105, 012001 (2008).

[110] H. Wijshoff, "The dynamics of the piezo inkjet printhead operation", Phys. Rep. 491, 77-177 (2010).

[111] H. P. Le, "Progress and trends in ink-jet printing technology", J. Imaging Sci. Technol. 42, 49-62 (1998).

[112] M. Singh, H. M. Haverinen, P. Dhagat, and G. E. Jabbour, "Inkjet printingprocess and its applications", Adv. Mater. 22, 673-85 (2010).

[113] J. U. Park, M. Hardy, S. J. Kang, K. Barton, K. Adair, D. K. Mukhopadhyay, C. Y. Lee, M. S. Strano, A. G. Alleyne, J. G. Georgiadis, P. M. Ferreira, and J. A. Rogers, "High-resolution electrohydrodynamic jet printing.", Nat. Mater. 6, 782-9 (2007).

[114] S. Mishra, K. L. Barton, A. G. Alleyne, P. M. Ferreira, and J. A. Rogers, "Highspeed and drop-on-demand printing with a pulsed electrohydrodynamic jet", J. Micromech. Microeng. 20, 095026 (2010).

[115] J. Eggers and T. F. Dupont, "Drop formation in a one-dimensional approximation of the navier-stokes equation", J. Fluid Mech. 262, 205-221 (1994).

[116] C. E. Brennen, Cavitation and bubble dynamics (Oxford University Press, New York) (1995). 
[117] W. van Hoeve, S. Gekle, J. H. Snoeijer, M. Versluis, M. P. Brenner, and D. Lohse, "Breakup of diminutive rayleigh jets", Phys. Fluids 22, 122-003 (2010).

[118] E. D. Wilkes, S. D. Phillips, and O. A. Basaran, "Computational and experimental analysis of dynamics of drop formation", Phys. Fluids 11, 3577-3598 (1999).

[119] A. U. Chen, P. K. Notz, and O. A. Basaran, "Computational and Experimental Analysis of Pinch-Off and Scaling”, Phys. Rev. Lett. 88, 174501 (2002).

[120] S. T. Thoroddsen, T. G. Etoh, and K. Takehara, "High-Speed Imaging of Drops and Bubbles", Annu. Rev. Fluid Mech. 40, 257-285 (2008).

[121] I. M. Hutchings, G. D. Martin, and S. D. Hoath, "High speed imaging and analysis of jet and drop formation”, J. Imaging Sci. Technol. 51, 438 (2007).

[122] J. de Jong, G. de Bruin, H. Reinten, M. van den Berg, H. Wijshoff, M. Versluis, and D. Lohse, "Air entrapment in piezo-driven inkjet printheads", J. Acoust. Soc. Am. 120, 1257 (2006).

[123] J. de Jong, R. Jeurissen, H. Borel, M. van den Berg, H. Wijshoff, H. Reinten, M. Versluis, A. Prosperetti, and D. Lohse, "Entrapped air bubbles in piezodriven inkjet printing: Their effect on the droplet velocity", Phys. Fluids 18, 121511 (2006).

[124] B. Beulen, J. de Jong, H. Reinten, M. van den Berg, H. Wijshoff, and R. van Dongen, "Flows on the nozzle plate of an inkjet printhead", Exp. Fluids 42, 217-224 (2007).

[125] R. Jeurissen, A. van der Bos, H. Reinten, M. van den Berg, H. Wijshoff, J. de Jong, M. Versluis, and D. Lohse, "Acoustic measurement of bubble size in an inkjet printhead", J. Acoust. Soc. Am. 126, 2184-2190 (2009).

[126] J. W. Shan, D. B. Lang, and P. E. Dimotakis, "Scalar concentration measurements in liquid-phase flows with pulsed lasers", Exp. Fluids 36, 268-273 (2004).

[127] T. Berg, J. Deppe, D. Michaelis, H. Voges, and S. Wissel, "Comparison of particle size and velocity investigations in sprays carried out by means of different measurement techniques", ICLASS (2006).

[128] M. W. Davidson and M. Abramowitz, "Optical microscopy", Encyclopedia of Imaging Science and Technology 2, 1106-1141 (2002). 
[129] C. T. Chin, C. Lancée, J. Borsboom, F. Mastik, M. E. Frijlink, N. de Jong, M. Versluis, and D. Lohse, "Brandaris 128: A digital 25 million frames per second camera with 128 highly sensitive frames", Rev. Sci. Instrum. 74, 50265034 (2003).

[130] M. Khomyakov, "Comparative evaluation of linear edge detection methods", Pattern Recognit. Image Anal. 22, 291-302 (2012).

[131] X. D. Shi, M. P. Brenner, and S. R. Nagel, "A cascade of structure in a drop falling from a faucet”, Science 265, 219-222 (1994).

[132] P. K. Notz and O. A. Basaran, "Dynamics and breakup of a contracting liquid filament”, J. Fluid Mech. 512, 223-256 (2004).

[133] C. Tang, P. Zhang, and C. K. Law, "Bouncing, coalescence, and separation in head-on collision of unequal-size droplets", Phys. Fluids 24, 022101 (2012).

[134] C. W. Visser, Y. Tagawa, C. Sun, and D. Lohse, "Microdroplet impact at very high velocity", Soft Matter 8, 10732-10737 (2012).

[135] S. T. Thoroddsen, T. G. Etoh, and K. Takehara, "Air entrapment under an impacting drop", J. Fluid Mech. 478, 125-134 (2003).

[136] D. B. van Dam and C. Le Clerc, "Experimental study of the impact of an inkjet printed droplet on a solid substrate", Phys. Fluids 16, 3403-3414 (2004).

[137] H. Dong, W. W. Carr, and J. F. Morris, "An experimental study of drop-ondemand drop formation”, Phys. Fluids 18, 072102 (2006).

[138] T. Driessen, P. Sleutel, F. Dijksman, R. Jeurissen, and D. Lohse, "Control of jet breakup by a superposition of two Rayleigh-Plateau-unstable modes", J. Fluid Mech. 749, 275-296 (2014).

[139] E. Lee, Microdrop Generation, Nano- and Microscience, Engineering, Technology and Medicine (Taylor \& Francis) (2002).

[140] F. A. Morrison, An Introduction to Fluid Mechanics, 625 (Cambridge University Press) (2013).

[141] K. Asano and K. Yatsuzuka, "Electrohydrodynamical behavior of charged droplets in air and its coalescence", IEEE Int. App. Soc. 2, 1402 - 1406 (1992).

[142] A. Castrejón-Pita, J. Castrejón-Pita, and I. Hutchings, "Breakup of Liquid Filaments", Phys. Rev. Lett. 108, 074506 (2012). 
[143] J. R. Lister and H. A. Stone, "Capillary breakup of a viscous thread surrounded by another viscous fluid", Phys. Fluids 10, 2758-2764 (1998).

[144] ESI-Group, “CFD-ACE+", https://www. esi-group.com.

[145] ANSYS, Inc., “ANSYS Fluent CFD”, http://www . ansys .com.

[146] S. Popinet, "An accurate adaptive solver for surface-tension-driven interfacial flows”, J. Comput. Phys. 228, 5838-5866 (2009).

[147] OpenCFD Ltd, "OpenFOAM", http: //www.openfoam.org.

[148] H. N. Oguz and A. Prosperetti, "Dynamics of Bubble-Growth and Detachment From a Needle", J. Fluid Mech. 257, 111-145 (1993).

[149] T. Glatzel, C. Litterst, C. Cupelli, T. Lindemann, C. Moosmann, R. Niekrawietz, W. Streule, R. Zengerle, and P. Koltay, "Computational fluid dynamics (CFD) software tools for microfluidic applications-A case study", Comput. Fluids 37, 218-235 (2008).

[150] C. W. Hirt and B. D. Nichols, "Volume of fluid (VOF) method for the dynamics of free boundaries", J. Comput. Phys. 39, 201-225 (1981).

[151] S. J. Cummins, M. M. Francois, and D. B. Kothe, "Estimating curvature from volume fractions", Comput. Struct. 83, 425-434 (2005). 


\section{Summary}

Inkjet technology is a very precise and highly flexible technique, which is well known for e.g. document printing. It is also gaining interest for applications such as manufacturing and decorative purposes. The technology is continuously being pushed to reach smaller drops and higher frequencies. Also the use of functional inks is challenging the printhead designs. To match the demands of this continuous development, a firm understanding of the drop formation process and the causes of inkjet defects are of great importance. Two of the most important methods used in inkjet printing research are high speed imaging and numerical modeling. In this thesis we aimed at creating methods and improving existing methods, to understand the formation of small scale droplets.

The acoustic domain and resulting droplet formation processes are well understood and characterized by various experimental techniques, however the behavior of the meniscus is a critical mediator between these regimes. The meniscus shape and motion vary between printhead designs, actuations and wetting conditions. The last decades several approaches have been suggested and implemented to experimentally obtain information on the fluid motion within inkjet nozzles. In Chapter 2 experimental methods are reviewed and novel techniques are introduced. The methods are compared in terms of accuracy and applicability.

Drop-on-demand (DoD) inkjet printing is well-characterized and a well-studied problem, but non-axisymmetric effects are typically ignored, while these effects can severely reduce the printhead performance and its stability. In Chapter 3 we first review non-axisymmetric droplet formation originating from geometrical effects. We then focus on the possibility that non-axisymmetric effects arise from surface instabilities of the meniscus; these include the Rayleigh-Taylor instability and a parametrically driven capillary instability. It is shown that the meniscus can become unstable beyond a critical acceleration threshold. A comparison with data extracted from high-speed recordings of the meniscus oscillations show that the acceleration threshold can indeed be exceeded, with higher order temporal frequencies exhibiting even larger accelerations. The experiments show that the duration at which the acceleration thresholds are exceeded are of the same order as the typical growth times of the instabilities. 
Next, we studied the behavior of the microdrops in flight. We made use of iLIF [10], which has a illumination time of $7 \mathrm{~ns}$ to record the fast and microscopic drops at high-resolution and without motion blur.

In Chapter 4 we report a microdroplet-based electrostatic generator operating by an acceleration-deceleration cycle termed ('ballistic' energy conversion), and show that this principle results in high efficiency. Water is accelerated by pumping it through a micronozzle to form a microjet breaking up into fast-moving charged droplets. The droplet kinetic energy is converted in electrical energy when the charged droplets decelerate in the electrical field that is formed between the membrane and the target. Using a high-speed photographic setup, we characterize the physical behavior of the microjet, the charged microdroplets formed and their subsequent aerodynamic flight towards the charge collection target, to calculate the efficiency of each conversion stage. We experimentally demonstrate conversion efficiencies of up to $48 \%$ with a $30 \mu \mathrm{m}$ diameter nozzle. An empirical model is created for the different stages, based on optical characterization and numerical simulations, from which we predict the optimal working regime. The model predicts a total energy conversion efficiency up to a $40 \%$ for a $10 \mu \mathrm{m}$ nozzle and $70 \%$ efficiency for a $30 \mu \mathrm{m}$ nozzle.

In Chapter 5 we introduce a method to extract the velocity profile inside a single droplet during drop formation. A novel experimental approach is used to capture two detailed images of the very same droplet with a small time delay. By accurately determining the volume distribution of the droplet the velocity within the droplet can be resolved. The obtained velocity profiles are compared to a numerical simulation based on the slender jet approximation of the Navier-Stokes equation were we find very good agreement.

Computational Fluid Dynamics(CFD) simulations are a meaningful contribution to inkjet research, since the drop formation can be predicted for a range of acoustic and fluidic parameters. Numerous numerical methods and codes have been developed to predict the drop formation process. To test the predictions of these models, typically drop benchmarks are performed were the models are compared to one another, without a priori knowledge of the expected formation behavior. In Chapter 6 we make use of a previous developed experimental technique to measure microdrops in flight. The data allows for a detailed comparison of the predictions of the numerical droplet formation models, by using measured drop profiles as input to the models and by comparing the predictions in subsequent time steps with the corresponding experimental recordings. We use two different droplet formation cases to compare 3 numerical codes, Gerris, Fluent and a slender jet model. These cases capture important features of the drop formation process, such as the moment of contraction, the drop velocity and the droplet size distribution. 


\section{Samenvatting}

Inkjet printen is een zeer flexibele techniek waarmee met grote precisie druppels inkt op papier geprint kunnen worden. Daarnaast kent de techniek vele andere toepassingen waarbij gedacht kan worden aan het 3D printen van objecten of aan het printen van cellen. Kleinere druppels die met een hogere frequentie geproduceerd kunnen worden zijn voorbeelden van de vereisten die deze nieuwe toepassingen stellen aan de huidige printtechniek. Om nieuwe materialen te kunnen printen is het van cruciaal belang dat het druppelvormingsproces tot in detail begrepen wordt. Hogesnelheidsfotografie is een belangrijke methode om met grote precisie het duppelvormingsproces te analyseren. Daarnaast hebben numerieke modellen die het druppelvormingsproces berekenen een grote toevoegende waarde doordat zij voorspellingen kunnen doen. In dit proefschrift laten we zien dat de combinatie van experimenten en modellen het begrip van het microscopische druppelvormingsproces sterk verhoogt.

Veel is al bekend over de vorm van de drukgolven in de printkop die nodig zijn voor het uitspuwen van de inkt. Daarnaast is er al veel kennis beschikbaar over de opbreking van de uitgespuwde inkt in een of meerdere druppels, het zogenaamde druppelvormingsproces. Er is echter nog maar weinig bekend over de dynamica van het vrije inktoppervlak tijdens het vormen van een druppel. Dit komt doordat het inktoppervlak zich in de spuitmond van de printkop bevindt waardoor deze lastig in beeld te brengen is. Daarbij komt dat de grootte van de spuitmond slechts 20 micrometer is en het vloeistofoppervlak met tientallen meters per seconde beweegt, erg klein en erg snelle bewegingen dus. Bovendien is het vloeistofoppervlak sterk gekromd waardoor deze licht in alle richtingen verstrooid. Al deze eigenschappen maken het bijzonder lastig om de dynamica van het vloeistofoppervlak te filmen. Hoofdstuk 2 geeft een overzicht van verschillende experimentele methodes waarmee naar de beweging van de meniscus gekeken kan worden. Naast de al uit de literatuur bekende methodes introduceren we 3 nieuwe methodes die allen met elkaar vergeleken worden aan de hand van de te meten fysische grootheden, de meetnauwkeurigheid en de toepasbaarheid van de meetmethode tijdens het printen.

Wanneer een inktstraal scheef door de spuitmond wordt afgevuurd heeft dit veelal een verminderde printkwaliteit tot gevolg. Het scheef afvuren van de inktstraal leidt 
er bovendien vaak toe dat de straal inkt opbreekt in vele kleine druppels in plaats van in één grote druppel. De kleine druppels bewegen in een andere richting dan waarin de grote druppel zou bewegen waardoor zij op de verkeerde plaats op het papier belanden. In hoofdstuk 3 worden oorzaken van niet-symmetrische druppelvorming besproken. We beginnen bij reeds bekende oorzaken, zoals productie fouten die leiden tot niet symmetrische printkoppen en zoals de verstoring van het druppelvormingsproces door vuildeeltjes in de spuitmond. Daarnaast wordt het onderzoek besproken dat we gedaan hebben naar het scheef afvuren van inkt door een niet symmetrische beweging van het inktoppervlak in de spuitmond dat kan ontstaan door een zogenaamde Rayleigh-Taylor instabiliteit of door parametrisch aangedreven instabiliteit. De minimale versnellingen van het vloeistofoppervlak die nodig zijn om deze instabiliteiten te doen groeien zijn theoretisch bepaald en ze zijn vergeleken met experimenteel bepaalde acceleraties, gemeten in een transparante printkop. We tonen aan dat de acceleraties groot genoeg zijn om een niet-symmetrische vloeistofoppervlak beweging te voeden, echter alleen als het vloeistofoppervlak vrij op en neer kan bewegen in de spuitmond.

Om onderzoek te kunnen doen naar de microscopisch grote uitgespuwde druppels moeten deze nauwkeurig in beeld gebracht worden. De hoge snelheid en de kleine afmeting van de druppels vereist erg korte belichtingstijden. We gebruiken een ultrahogesnelheidsfotografie systeem dat gebruikt maakt van een laser om de druppels te fotograferen. Door met een laser lichtflits van slechts $7 \mathrm{~ns}$ een fluorescerend scherm te belichten, wordt een lichtflits verkregen die geschikt is voor belichting van microscopische druppels. In hoofdstuk 4 laten we zien dat het fotograferen met deze techniek de mogelijk biedt om heel precies de vertraging van elektrisch geladen druppels in een elektrisch veld te meten. De kinetische energie die verloren gaat door de druppels te vertragen wordt omgezet in elektrische energie. Op deze manier kan de energie die in de druppels gestopt is, bij het door de spuitmond persen van de vloeistof, omgezet worden in elektrische energie. Metingen laten zien dat een spuitmond met een diameter van $30 \mu \mathrm{m}$ een energieconversie van $48 \%$ gehaald kan worden. Een deel van de energie gaat namelijk verloren doordat de druppels luchtwrijving ondervinden. Er is vervolgens een empirisch model gemaakt om het optimale werkbereik van de generator te vinden. Hiermee is bepaald dat de maximale efficintie $40 \%$ is voor een spuitmond met een diameter van $10 \mu \mathrm{m}$ en $70 \%$ is voor een $30 \mu \mathrm{m}$ spuitmond.

In hoofdstuk 5 is een methode ontwikkeld om de snelheid van de vloeistofstroming in een straal inkt te bepalen nadat deze uit de spuitmond geschoten is. Deze inktstraal trekt samen tot een inktdruppel, het exacte proces van samentrekken en de daarmee gemoeide stomingsnelheden in de inktdruppel kunnen met de meetmethode 
bepaald worden. De methode analyseert twee hogesnelheidsfoto's die 600 nanoseconden na elkaar gemaakt worden. Het ultra-hogesnelheidsfotografiesysteem besproken in hoofdstuk 4 werd hiervoor gebruikt met als toevoeging een dubbele laser die twee flitsen kan produceren en een fotocamera die kort na elkaar twee afbeeldingen kan maken. De volume verdeling in de inkt druppel is bepaald door in de lengte richting afgeknotte conussen in de straal te passen. Uit deze tijdsafhankelijke volumeverdeling kan de snelheid in de druppel worden achterhaald. De gemeten snelheidsverdelingen van twee experimenteel verschillende druppelvormingsprocessen zijn vergeleken met een computer model dat de druppelvorming uitrekent. De gemeten druppelvorm en het gemeten snelheidsprofiel worden ingevoerd in het model en worden op numerieke wijze de druppelvorm en vloeistofsnelheden berekend op latere tijdstappen. Het vergelijk tussen de tijdafhankelijke gemeten druppelvorming en berekende druppelvorming laat een goede overeenkomst zien.

Computer modellen zijn van grote toegevoegde waarde voor het onderzoek naar druppelvormingsprocessen in de inkjet technologie. De gevormde druppels kunnen worden gesimuleerd voor een groot bereik van aansturing en vloeistof parameters. Er zijn een groot aantal numerieke modellen ontwikkeld die gebruikt worden voor het berekenen van druppelvorming. Om deze modellen te verifiëren worden vaak verschillende computer modellen vergeleken zonder kennis over de te verwachten druppelvorming. In hoofdtuk 6 wordt gebruik gemaakt van de in hoofdstuk 5 ontwikkelde methode om de berekeningen van verschillende numerieke computer modellen te vergelijken. We vergelijken de voorspellingen van de computer modellen genaamd 'Gerris', 'Fluent' en het 'slender-jet' model voor een samentrekkende druppel en een druppel die opbreekt in kleinere druppels. In het vergelijk vinden we welke verschillen optreden door de aanwezigheid van lucht rond en ook tussen de druppels. 


\section{Acknowledgements}

The work in this thesis is part of the research programme Contact Line Control during Wetting and Dewetting (CLC) of the Foundation for Fundamental Research on Matter (FOM), which is financially supported by the Netherlands Organisation for Scientific Research (NWO). The CLC programme is co-financed by Océ Technologies BV., Venlo and ASML Netherlands BV, Veldhoven. The work was carried out at both the Physics of Fluids group of the Faculty of Science and Technology of the University of Twente and at Océ Technologies in Venlo.

First of all I want to thank my promotors Detlef and Michel for giving me the opportunity to perform my PhD research in the Physics of Fluids group. I am very grateful for all the input on the thesis and for the support getting there.

Of course my project would not have developed as it did, or would even have existed, without the input and support of the crew at Océ. Marc and Herman, thanks you very much for the respectively discussions on analysis/lab related topics and on numerical topics. Hans, you never seem to run out of ideas and your enthusiasm is very stimulating. Arjan v/d Bos you were not only my supervisor during my masters assignment and all-round discussion partner since then, but you also introduced the iLIF to the group and Océ, which allowed for many of the detailed recordings in this thesis.

I would like to thank the many people with which I had the opportunity to work with on Océ related projects. Theo, Roger and Erik-Jan thanks for the nice discussions we had together. Furthermore I wish Arjan Fraters who more recently started his $\mathrm{PhD}$ and continuous the research on jetting defects, all the best and nice results.

During my research I got to work with many different $\mathrm{PhD}$ students which were involved in producing and monitoring micro droplets: Fede, Kike, Yanbo, Diederik, Patrick and Alexander I enjoyed working with you in the lab. I supervised two students on their bachelor assignment in inkjet printing, Maurits and Niels, thanks for your commitment during this project. 
Chao, although we were not directly involved in a project, I was always welcome in your office for advice on imaging or for less work related topics, and I appreciated it very much.

I am thankful for my office mates, with made office 248 feel like home for the many years we have spend there together: Tess, (Joost), Theo, Antonin, Sander, Marie-Jean and Rodolfo, I think we were the only office for many years in which everybody was able to run decent a cascade with juggling balls. Next to the many entertaining discussions we have had, I also learned a great deal from you. Tess although we shared all of our study years together, I think we never really got to know each other until we shared an office. I am very glad we did so, and finally did some serious club passing sessions.

Also I would like to thank the people of office 214, which felt like my second office: Erik, Arjan, Tim, Guillaume and Pascal thank you for the many discussions and help in and around the lab. I was happy to work in the lab-next-door, and to join you in the activities outside office hours like the POF "borrel"-committee or the welding course.

Non of the work in our research group would go this smooth without the supporting help of the technical staff, Gert-Wim and Martin. Bas thanks a million for saving me out multiple times when the hardware of my macbook proved to be not as robust as one would hope for. And of course thanks to Joanita, who seemingly could solve any problem with a single phone call.

I would like to say thanks to the many students, PhD-students, post-docs and staff which joined me in a game of Futsal, to try to defend the honors of POF United. We might not have won all of the matches, but I enjoyed it a lot.

Shortly after I moved to Enschede, I became a fanatic juggler, which was mainly due to the good atmosphere in our juggling association CatchUp. I want to thank the many friends I made over the year in this open minded group. Vincent, Menno, Tess, Jasper, Geert, Alje, Johan, Jeroen, Robert, Tom, Rodolfo and many more, I want to thank you all for the numerous good juggling sessions, games and discussions we had over the years.

Ik wil mijn familie bedanken voor de ondersteuning die zij mij hebben gegeven tijdens mijn promotieonderzoek. Mijn ouders: mam en Henk, zelfs na meerdere jaren in andere steden te hebben gewoond, waren jullie in staat me in Hoogeveen thuis te laten voelen en kon ik altijd op steun rekenen wanneer dat nodig was. Pap, hoewel je mij niet hebt mogen zien opgroeien, en misschien wel juist daarom, heb ik altijd 
mijn best gedaan zodat je trots op me zou zijn geweest. Ik hou van jullie.

Mijn zus Daphne en Ronald, jullie deur stond altijd open en ik wil jullie bedanken voor de vele weekenden die we bij jullie en de kinderen mogen doorbrengen.

Natuurlijk zou niets zo zijn gelopen zonder Anne, de liefde van mijn leven, met wie ik kort voor het begin van mijn promotieonderzoek ben gaan samenwonen in Deventer. Het vele dagelijkse reizen waren het alleen al waard om 's avonds jou lieve lach te mogen zien. Ik weet dat het een enorm offer van je was om definitief zo ver van je familie en vrienden te bijven wonen, maar ik ben je er nog elke dag dankbaar voor. Ich liebe dich mein Schatz.

Inmiddels zijn we een gezin van drie en is Lotta de liefste dochter die ik mij voor kan stellen. Het vaderschap geeft mij meer trots en blijdschap dan ik ooit had gedacht. Ik ben dan ook erg gelukkig dat wij binnenkort onze tweede dochter mogen begroeten.

Mark-Jan,

Deventer, January 2015 


\section{About the author}

Mark-Jan van der Meulen was born on Februari 1st, 1986 in Apeldoorn, the Netherlands. He graduated from high school (VWO) at "Het Roelof van Echten college" in Hoogeveen in 2004. In the same year he started studying Applied Physics at the University of Twente in Enschede. During his studies he did an internship at the "Institut für Biomedizinische Optik" of the "Universität zu Lübeck" in Germany, where he worked on mechanisms of Laser Microdissection and Pressure Catapulting (LMPC) for tissue with Prof. dr. Alfred Vogel. In 2011 he received his master's degree in the Physics of Fluids group with Prof. dr. Detlef Lohse and dr. Chao Sun on "3D visualization of a meniscus in piezo inkjet printing". Thereafter he started his $\mathrm{PhD}$ research in the Physics of Fluids group, on the subject of meniscus motion and drop formation in inkjet printing. This project was supervised by Prof. dr. Detlef Lohse and Prof. dr. Michel Versluis, and was conducted in close cooperation with Océ Technologies BV. 


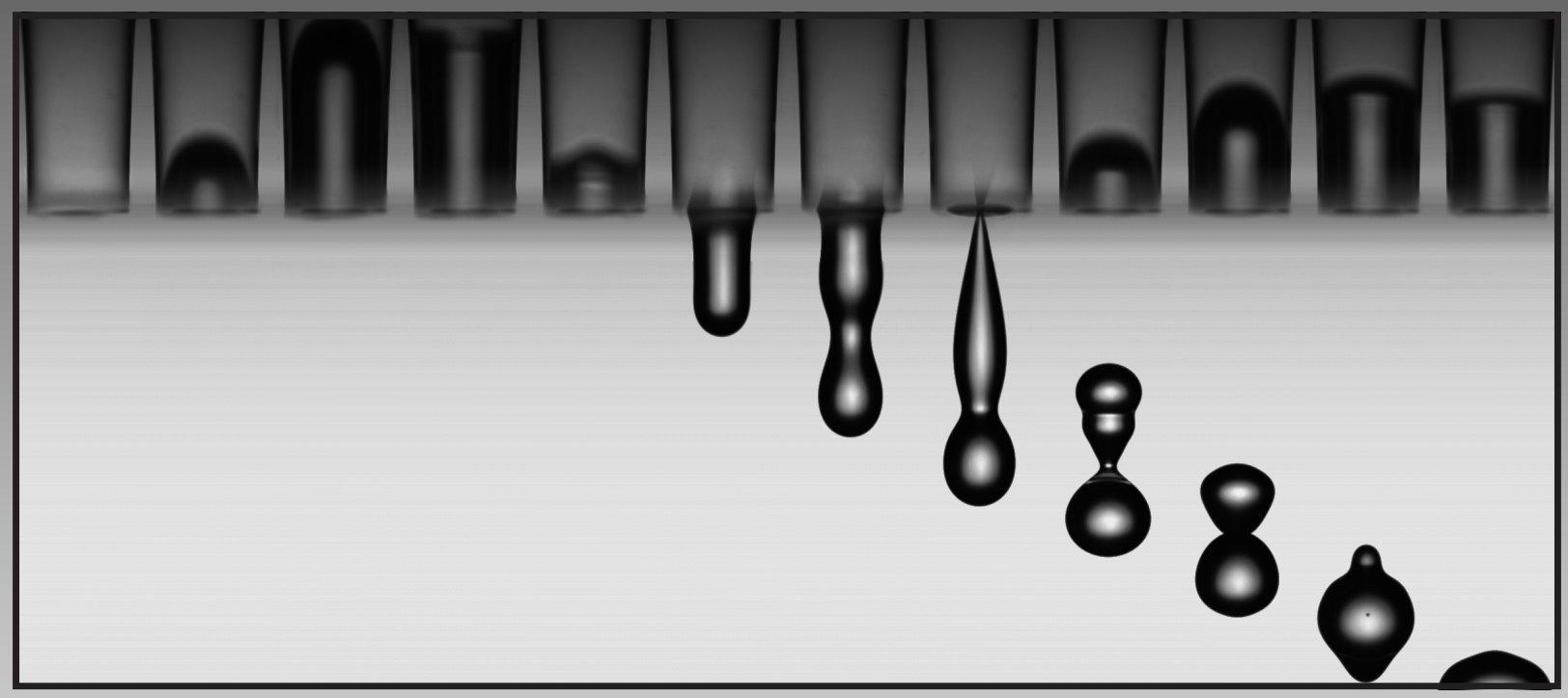

